\title{
Diastereo- and Atroposelective Synthesis of Bridged Biaryls \\ Bearing an Eight-Membered Lactone through an \\ Organocatalytic Cascade
}

Shenci Lu, Jun Yang Ong, Hui Yang, Si Bei Poh, Xi Liew, Chwee San Deborah Seow,

Ming Wah Wong,* and Yu Zhao*

\section{Supporting Information}

$\begin{array}{ll}\text { 1. General information } & \text { S2 }\end{array}$

2. Synthesis of the substrates $\quad$ S3

3. Synthesis of benzofuran-derived bridged biaryls $\quad$ S25

$\begin{array}{ll}\text { 4. Characterization of benzofuran-derived bridged biaryls } & \text { S27 }\end{array}$

5. Synthesis of indole-derived bridged biaryls $\quad$ S42

6. Characterization of indole-derived bridged biaryls $\quad$ S44

7. The synthesis of substituted bridged biaryls and axially chiral biaryls $\quad$ S55

8. Crystal structures of $\mathbf{4 b}, \mathbf{6} \mathbf{g}$ and $\mathbf{4 u} \quad \mathrm{S} 58$

9. Investigation of side reaction pathway $\quad$ S62

10. References $\quad$ S64

11. NMR spectra $\quad$ S65

$\begin{array}{ll}\text { 12. Computational data } & \text { S107 }\end{array}$ 
1. General information. ${ }^{\mathbf{1}} \mathbf{H}$ and ${ }^{13} \mathbf{C}$ NMR spectra were recorded on a Bruker AMX500 $(500 \mathrm{MHz})$ spectrometer. Chemical shifts were reported in parts per million (ppm), and the residual solvent peak was used as an internal reference: ${ }^{1} \mathrm{H}$ (chloroform $\delta$ 7.26; DMSO $\delta 2.50$; acetone- $d_{6} \delta 2.05$ ), ${ }^{13} \mathrm{C}$ (chloroform $\delta 77.0$; DMSO $\delta 39.5$; acetone- $d_{6} \delta 205.87,30.60$ ). Data are reported as follows: chemical shift, multiplicity $(\mathrm{s}=$ singlet, $\mathrm{d}=$ doublet, $\mathrm{t}=$ triplet, $\mathrm{q}=$ quartet, $\mathrm{m}=$ multiplet, $\mathrm{br}=$ broad), coupling constants $(\mathrm{Hz})$ and integration. Melting point (MP) was obtained on Buchi B-540. For thin layer chromatography (TLC), Merck pre-coated TLC plates (Merck 60 F254) were used, and compounds were visualized with a UV light at 254nm. High resolution mass spectra (HRMS) were obtained on a Finnigan/MAT 95XL-T spectrometer. Optical rotations were recorded on an mrc AP81 automatic polarimeter. Enantiomeric excesses (ee) were determined by HPLC analysis on Agilent HPLC units, including the following instruments: pump, LC-20AD; detector, SPD-20A; column, Chiralcel OD-H, Chiralpak AD-H, IA, IC or ID.

All reactions were carried out under nitrogen atmosphere. All commercially available reagents listed below were used as received for the reactions without any purification. Hydrocinnamaldehyde, isovaleraldehyde, $(E)$-pent-2-enal, $(E)$-hex-2-enal, $(E)$-hept-2-enal , Ethynylmagnesium bromide solution $0.5 \mathrm{M}$ in THF, $N, N$-diisopropylethylamine (DIPEA) and 1,8-Diazabicyclo[5.4.0]undec-7-ene (DBU) were purchased from Alfa Aeser. Liquid reagents were handled with a micropipette. THF was dried on alumina columns using a solvent dispensing system. The NHC precursors were synthesized following the reported procedure ${ }^{1-2}$. 2-Benzoylisovaleraldehyde ${ }^{3}$ was synthesized by the reported procedure. The starting materials bis(2-hydroxyaryl)methanone ${ }^{4}$ and (2-aminoaryl)(2-hydroxyaryl)methanone ${ }^{5}$ were synthesized by the reported procedure. 


\section{Synthesis of the substrates.}

\subsection{General procedure A: Ruthenium catalyzed orthohydroxylation of aryl}

ketones.

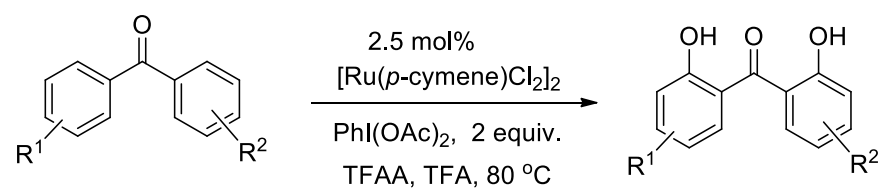

To a $100 \mathrm{ml}$ sealed-tube were added ketone ( $3 \mathrm{mmol}, 1.0$ equiv), oxidants $\mathrm{PhI}(\mathrm{OAc})_{2}$ (2.0 equiv), $\left[\mathrm{Ru}(p \text {-cymene }) \mathrm{Cl}_{2}\right]_{2}(0.025$ equiv), TFAA $(6 \mathrm{ml})$ and TFA $(14 \mathrm{ml})$. The tube was sealed and heated at $80{ }^{\circ} \mathrm{C}$. The reaction was monitored by TLC (petroleum ether: toluene $=$ 3:1). After completion of the reaction, dichloromethane was added to dilute the reaction mixture and saturated aqueous $\mathrm{NaHCO}_{3}$ was added to neutralize TFA and TFAA. Then the organic layer was dried over anhydrous $\mathrm{Na}_{2} \mathrm{SO}_{4}$ and concentrated on rotavapor under reduced pressure. Finally, the residue was purified by silica gel column chromatography to give the desired product.

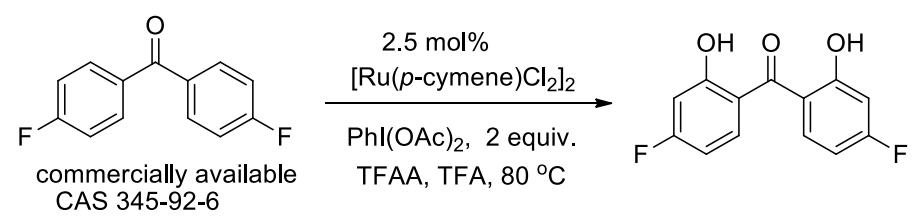

Following the general procedure A. $60 \%$ yield. Yellow solid: MP: $83-84{ }^{\circ}$ C. ${ }^{1}$ H NMR $(500$ $\left.\mathrm{MHz}, \mathrm{CDCl}_{3}\right) \delta 10.91(\mathrm{~d}, J=1.6 \mathrm{~Hz}, 2 \mathrm{H}), 7.63(\mathrm{dd}, J=8.9,6.4 \mathrm{~Hz}, 2 \mathrm{H}), 6.81(\mathrm{dd}, J=10.2,2.6$ $\mathrm{Hz}, 2 \mathrm{H}), 6.70(\mathrm{td}, J=8.4,2.6 \mathrm{~Hz}, 2 \mathrm{H}) .{ }^{13} \mathbf{C}$ NMR $\left(125 \mathrm{MHz}, \mathrm{CDCl}_{3}\right) \delta 200.03,167.26(\mathrm{~d}, J=$ $257.7 \mathrm{~Hz}), 164.34(\mathrm{~d}, J=14.1 \mathrm{~Hz}), 135.01(\mathrm{~d}, J=11.5 \mathrm{~Hz}), 116.53(\mathrm{~d}, J=2.6 \mathrm{~Hz}), 107.29(\mathrm{~d}, J$ $=22.8 \mathrm{~Hz}), 105.60(\mathrm{~d}, J=24.0 \mathrm{~Hz}) . \mathbf{H R M S}($ ESI $) \mathrm{m} / \mathrm{z}$ Calcd for $\left[\mathrm{C}_{13} \mathrm{H}_{8} \mathrm{~F}_{2} \mathrm{NaO}_{3}, \mathrm{M}+\mathrm{Na}\right]^{+}$: 293.0334; Found 293.0336.

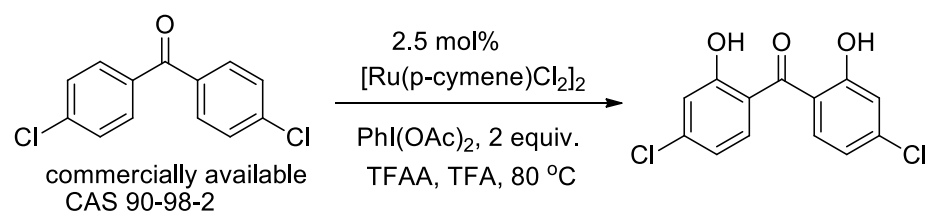

Following the general procedure A. $65 \%$ yield.Yellow solid: MP: $103-104{ }^{\circ} \mathrm{C} .{ }^{1} \mathbf{H}$ NMR (400 $\left.\mathrm{MHz}, \mathrm{CDCl}_{3}\right) \delta 10.65(\mathrm{~s}, 2 \mathrm{H}), 7.50(\mathrm{~d}, J=8.6 \mathrm{~Hz}, 2 \mathrm{H}), 7.11(\mathrm{~s}, 2 \mathrm{H}), 6.93(\mathrm{~d}, J=8.6 \mathrm{~Hz}, 2 \mathrm{H})$; 
${ }^{13}$ C NMR (100 MHz, CDC13) $\delta 200.65,162.54,142.36,133.54,119.82,118.91,118.33$.

HRMS (ESI) $\mathrm{m} / \mathrm{z}$ Calcd for $\left[\mathrm{C}_{13} \mathrm{H}_{8} \mathrm{Cl}_{2} \mathrm{NaO}_{3}, \mathrm{M}+\mathrm{Na}\right]^{+}:$304.9742; Found 304.9746.

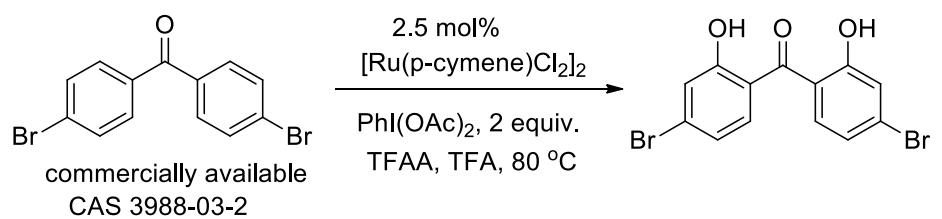

Following the general procedure A. $65 \%$ yield. Yellow solid: MP: $160-161{ }^{\circ} \mathrm{C} . \quad{ }^{1} \mathbf{H}$ NMR $(500$ $\left.\mathrm{MHz}, \mathrm{CDCl}_{3}\right) \delta 10.63(\mathrm{~s}, 2 \mathrm{H}), 7.45(\mathrm{~d}, J=8.6 \mathrm{~Hz}, 2 \mathrm{H}), 7.32(\mathrm{~s}, 2 \mathrm{H}), 7.12(\mathrm{~d}, J=8.5 \mathrm{~Hz}, 2 \mathrm{H})$. ${ }^{13} \mathrm{C}$ NMR $\left(125 \mathrm{MHz}, \mathrm{CDCl}_{3}\right) \delta 200.88,162.18,133.47,130.91,122.65,122.04,118.44$.

HRMS (ESI) m/z Calcd for $\left[\mathrm{C}_{13} \mathrm{H}_{8} \mathrm{Br}_{2} \mathrm{NaO}_{3}, \mathrm{M}+\mathrm{Na}\right]^{+}:$392.8732; Found 392.8736.

\subsection{General procedure B: Synthesis of bis(2-methoxyaryl)methanones and} synthesis of bis(2-hydroxyaryl)methanones.

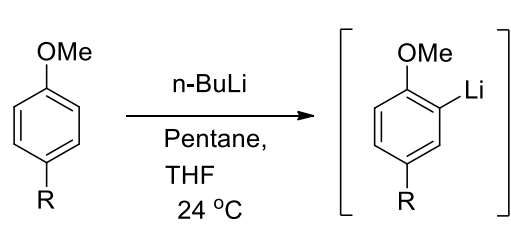

$\mathrm{R}=\mathrm{Me}$ or $\mathrm{C}\left(\mathrm{CH}_{3}\right)_{3}$<smiles>COc1cccc(C)c1</smiles>

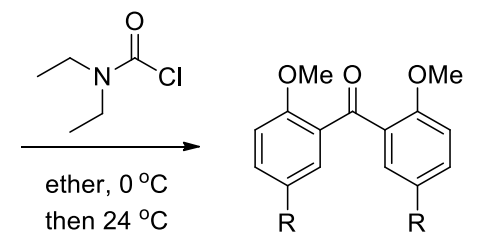

then $24^{\circ} \mathrm{C}$

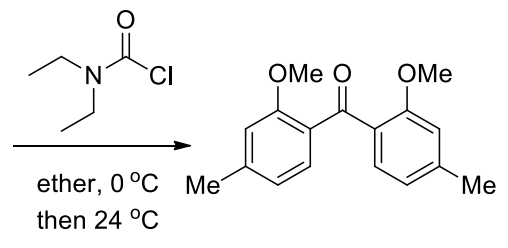

To a clean, dry roundbottom flask equipped with a large stir bar was added anhydrous Pentane (1.6 mL), anisole derivative (15.0 mmol), and THF (3.65 mL, $45.0 \mathrm{mmol})$ (3 equiv.) under nitrogen atmosphere. Next $\mathrm{n}-\mathrm{BuLi}$ (2 $\mathrm{M}$ in hexanes) $(7.9 \mathrm{~mL}, 15.0 \mathrm{mmol})$ was carefully added with vigorous stirring. The reaction was allowed to stir at $24{ }^{\circ} \mathrm{C}$ or $60{ }^{\circ} \mathrm{C}$ for $36 \mathrm{~h}$. The slightly cloudy yellow solution was cooled to $0{ }^{\circ} \mathrm{C}$ using an ice bath. The solution of diethylcarbamyl chloride $(0.90 \mathrm{~mL}, 7.5 \mathrm{mmol})$ in $10 \mathrm{~mL}$ of dry diethyl ether was added dropwise (extremely vigorous reaction!) with rapid stirring. At the end of the addition, the 
yellow solution had turned into a white creamy suspension. The cooling bath was removed and the reaction mixture stirred for one hour at room temperature, after which $10 \mathrm{~mL}$ of $\mathrm{NH}_{4} \mathrm{Cl}$ solution was carefully added. The mixture was extracted with ethyl acetate $(3 \times 20$ $\mathrm{mL}$ ), and the combined extracts were washed with brine and dried over sodium sulphate. The crude was purified by chromatography on silica gel (10:1 hexane/ethyl acetate), and gave the title compound.

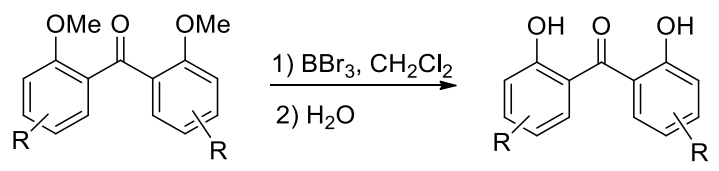

(Reaction was proceed under $-78^{\circ} \mathrm{C}$ ) To a solution of bis(2-methoxyaryl)methanones $(1.3 \mathrm{mmol})$ in dichloromethane was added boron tribromide $(1.3 \mathrm{M}, 4.0 \mathrm{~mL}, 5.2 \mathrm{mmol})$ in dropwise manner. The mixture was then stirred under room temperature overnight. The reaction was quenched and neutralized with saturated sodium bicarbonate solution to $\sim \mathrm{pH} 7$, followed by addition of water to separate the dichloromethane layer. The collected aqueous layer then extracted with ethyl acetate $(3 \times 10 \mathrm{~mL})$, and the combined extracts were washed with brine and dried over sodium sulphate. The crude was purified by chromatography on silica gel (10:1 hexane/ethyl acetate), and gave the title compound.<smiles>COc1ccc(OC)c(C(=O)c2cc(C)ccc2OC)c1</smiles>

Following the general procedure B. $\quad 70 \%$ yield. White solid. MP: $112-113{ }^{\circ} \mathrm{C} .{ }^{1} \mathrm{H}$ NMR $(500$ $\left.\mathrm{MHz}, \mathrm{CDCl}_{3}\right) \delta 7.34(\mathrm{~d}, J=2.4 \mathrm{~Hz}, 2 \mathrm{H}), 7.25(\mathrm{dd}, J=8.4,2.4 \mathrm{~Hz}, 2 \mathrm{H}), 6.83(\mathrm{~d}, J=8.4 \mathrm{~Hz}, 2 \mathrm{H})$, $3.66(\mathrm{~s}, 6 \mathrm{H}), 2.32(\mathrm{~s}, 6 \mathrm{H}) .{ }^{13} \mathrm{C} \mathrm{NMR}\left(125 \mathrm{MHz}, \mathrm{CDCl}_{3}\right) \delta 195.66,156.36,133.02,130.68$, $130.12,129.62,111.57,55.91,20.37$. HRMS (ESI) $\mathrm{m} / \mathrm{z}$ Calcd for $\left[\mathrm{C}_{17} \mathrm{H}_{18} \mathrm{NaO}_{3}, \mathrm{M}+\mathrm{Na}\right]^{+}$: 293.1148; Found 293.1144. 


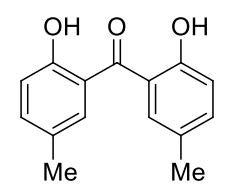

Following the general procedure B. $\quad 86 \%$ yield. Yellow solid: MP: $102-103{ }^{\circ} \mathrm{C} .{ }^{1} \mathbf{H}$ NMR (500 $\left.\mathrm{MHz}, \mathrm{CDCl}_{3}\right) \delta 10.42(\mathrm{~s}, 2 \mathrm{H}), 7.42(\mathrm{~d}, J=2.3 \mathrm{~Hz}, 2 \mathrm{H}), 7.35(\mathrm{dd}, J=8.5,2.3 \mathrm{~Hz}, 2 \mathrm{H}), 7.02(\mathrm{~d}, J$ $=8.5 \mathrm{~Hz}, 2 \mathrm{H}), 2.34(\mathrm{~s}, 6 \mathrm{H}) .{ }^{13} \mathrm{C} \mathbf{N M R}\left(125 \mathrm{MHz}, \mathrm{CDCl}_{3}\right) \delta 202.35,159.54,136.76,132.73$, $128.00,119.71,118.30,20.57$. HRMS (ESI) $\mathrm{m} / \mathrm{z}$ Calcd for $\left[\mathrm{C}_{15} \mathrm{H}_{14} \mathrm{NaO}_{3}, \mathrm{M}+\mathrm{Na}\right]^{+}$: 263.0835; Found 263.0832.

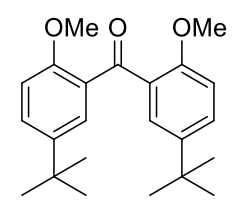

Following the general procedure B. $\quad 70 \%$ yield. White solid. MP: $123-124{ }^{\circ} \mathrm{C} .{ }^{1} \mathbf{H}$ NMR (500 $\left.\mathrm{MHz}, \mathrm{CDCl}_{3}\right) \delta 7.55(\mathrm{~d}, J=2.6 \mathrm{~Hz}, 2 \mathrm{H}), 7.46(\mathrm{ddd}, J=8.7,2.6,1.0 \mathrm{~Hz}, 2 \mathrm{H}), 6.88(\mathrm{dd}, J=8.6$, $0.9 \mathrm{~Hz}, 2 \mathrm{H}), 3.68$ (s, 6H), 1.33 (s, 18H). ${ }^{13} \mathbf{C}$ NMR (125 MHz, $\left.\mathrm{CDCl}_{3}\right) \delta 195.91,156.27$, $142.91,129.62,129.26,127.47,111.35,55.89,34.14,31.41$. HRMS (ESI) m/z Calcd for $\left[\mathrm{C}_{23} \mathrm{H}_{30} \mathrm{NaO}_{3}, \mathrm{M}+\mathrm{Na}\right]^{+}:$377.2087; Found 377.2089.<smiles>CC(C)(C)c1ccc(O)c(C(=O)c2cc(C(C)(C)C)ccc2O)c1</smiles>

Following the general procedure B. $\quad 88 \%$ yield. Yellow solid. MP: $\quad 103-104{ }^{\circ} \mathrm{C} . \quad{ }^{1} \mathbf{H}$ NMR $\left(500 \mathrm{MHz}, \mathrm{CDCl}_{3}\right) \delta 10.50(\mathrm{~s}, 2 \mathrm{H}), 7.70-7.57(\mathrm{~m}, 4 \mathrm{H}), 7.08(\mathrm{dd}, J=9.4,1.2 \mathrm{~Hz}, 2 \mathrm{H}), 1.33$ (s, 18H). ${ }^{13} \mathbf{C}$ NMR $\left(125 \mathrm{MHz}, \mathrm{CDCl}_{3}\right) \delta 202.91,159.58,141.50,133.42,129.16,119.41$, $118.16,34.21,31.39$. HRMS (ESI) $\mathrm{m} / \mathrm{z}$ Calcd for $\left[\mathrm{C}_{21} \mathrm{H}_{26} \mathrm{NaO}_{3}, \mathrm{M}+\mathrm{Na}\right]^{+}:$349.1774; Found 349.1778.

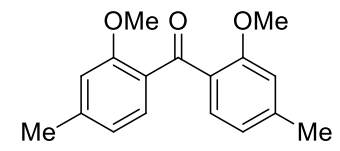

Following the general procedure B. $\quad 67 \%$ yield. White solid. MP: $102-103{ }^{\circ} \mathrm{C} .{ }^{1} \mathbf{H}$ NMR (500 $\left.\mathrm{MHz}, \mathrm{CDCl}_{3}\right) \delta 7.41(\mathrm{~d}, J=7.7 \mathrm{~Hz}, 2 \mathrm{H}), 6.80(\mathrm{dd}, J=8.0,1.2 \mathrm{~Hz}, 2 \mathrm{H}), 6.74(\mathrm{~s}, 2 \mathrm{H}), 3.69(\mathrm{~s}$, 
6H), $2.41(\mathrm{~s}, 6 \mathrm{H}) .{ }^{13} \mathrm{C}$ NMR $\left(125 \mathrm{MHz}, \mathrm{CDCl}_{3}\right) \delta 194.84,158.41,143.23,130.69,127.77$, $121.03,112.31,55.70,21.91$. HRMS (ESI) m/z Calcd for $\left[\mathrm{C}_{17} \mathrm{H}_{18} \mathrm{NaO}_{3}, \mathrm{M}+\mathrm{Na}\right]^{+}$: 293.1148; Found 293.1146.

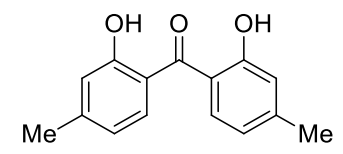

Following the general procedure B. $86 \%$ yield. Yellow solid: MP: $108-109{ }^{\circ} \mathrm{C} .{ }^{1} \mathbf{H}$ NMR (500 $\left.\mathrm{MHz}, \mathrm{CDCl}_{3}\right) \delta 10.79(\mathrm{~s}, 2 \mathrm{H}), 7.53(\mathrm{~d}, J=8.1 \mathrm{~Hz}, 2 \mathrm{H}), 6.91(\mathrm{~d}, J=1.5 \mathrm{~Hz}, 2 \mathrm{H}), 6.77(\mathrm{dd}, J=$ 8.2, 1.6 Hz, 2H), $2.41(\mathrm{~s}, 6 \mathrm{H}) .{ }^{13} \mathrm{C} \mathrm{NMR}\left(125 \mathrm{MHz}, \mathrm{CDCl}_{3}\right) \delta 201.40,161.91,147.40,132.91$, $120.05,118.65,117.53,21.92$. HRMS (ESI) m/z Calcd for $\left[\mathrm{C}_{15} \mathrm{H}_{14} \mathrm{NaO}_{3}, \mathrm{M}+\mathrm{Na}\right]^{+}$: 263.0835; Found 263.0838.
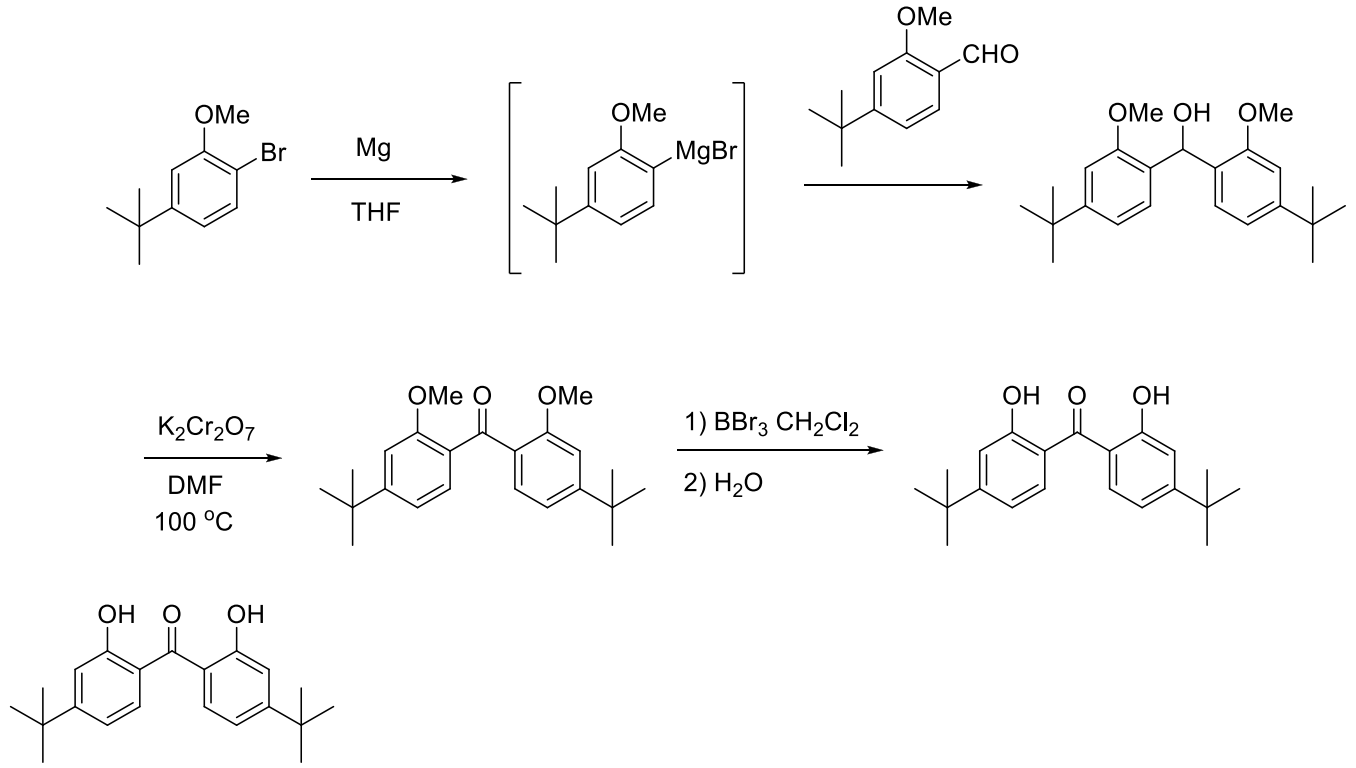

Following the reported procedure. ${ }^{6}$ Yellow solid: MP: $127-128{ }^{\circ} \mathrm{C} . \quad{ }^{1} \mathbf{H}$ NMR $(500 \mathrm{MHz}$, $\left.\mathrm{CDCl}_{3}\right) \delta 10.83(\mathrm{~s}, 2 \mathrm{H}), 7.62(\mathrm{~d}, J=8.4 \mathrm{~Hz}, 2 \mathrm{H}), 7.12(\mathrm{~d}, J=1.7 \mathrm{~Hz}, 2 \mathrm{H}), 6.99(\mathrm{dt}, J=8.5,1.6$ $\mathrm{Hz}, 2 \mathrm{H}), 1.37(\mathrm{~d}, J=1.4 \mathrm{~Hz}, 18 \mathrm{H}) .{ }^{13} \mathbf{C}$ NMR $\left(125 \mathrm{MHz}, \mathrm{CDCl}_{3}\right) \delta 201.18,161.86,160.38$, $132.78,117.42,116.42,115.29,35.34,30.84$. HRMS (ESI) $\mathrm{m} / \mathrm{z}$ Calcd for $\left[\mathrm{C}_{21} \mathrm{H}_{26} \mathrm{NaO}_{3}, \mathrm{M}\right.$ $+\mathrm{Na}]^{+}:$349.1774; Found 349.1776. 

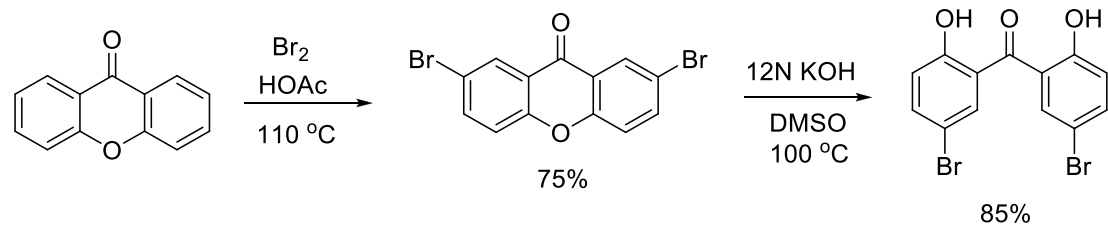<smiles>O=C(c1cc(Br)ccc1O)c1cc(Br)ccc1O</smiles>

Following the reported procedure. ${ }^{7} \quad$ Yellow solid: MP: $\quad 131-132{ }^{\circ} \mathrm{C} . \quad{ }^{1} \mathrm{H}$ NMR $(500 \mathrm{MHz}$, $\left.\mathrm{CDCl}_{3}\right) \delta 10.32(\mathrm{~s}, 2 \mathrm{H}), 7.70(\mathrm{~s}, 2 \mathrm{H}), 7.64(\mathrm{dd}, J=8.8,2.5 \mathrm{~Hz}, 2 \mathrm{H}), 7.03(\mathrm{~d}, J=8.8 \mathrm{~Hz}, 2 \mathrm{H}) .{ }^{13} \mathrm{C}$ NMR $\left(125 \mathrm{MHz}, \mathrm{CDCl}_{3}\right) \delta 199.89,160.58,139.00,134.43,120.80,120.76,111.02$.<smiles>O=c1c2ccccc2oc2ccccc12</smiles><smiles>O=c1c2ccccc2oc2ccc([N+](=O)[O-])cc12</smiles>

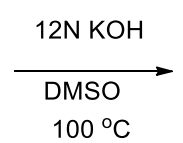<smiles>O=C(c1ccccc1O)c1cc([N+](=O)[O-])ccc1O</smiles>

$90 \%$<smiles>O=C(c1ccccc1O)c1cc([N+](=O)[O-])ccc1O</smiles>

Following the reported procedure. ${ }^{7} \quad$ Yellow solid: MP: $\quad 157-158{ }^{\circ} \mathrm{C} . \quad{ }^{1} \mathrm{H}$ NMR $(500 \mathrm{MHz}$, Acetone- $\left.d_{6}\right) \delta 11.07(\mathrm{br}, 2 \mathrm{H}), 8.40(\mathrm{~s}, 1 \mathrm{H}), 8.38(\mathrm{~d}, J=2.6 \mathrm{~Hz}, 1 \mathrm{H}), 7.60(\mathrm{t}, J=7.8 \mathrm{~Hz}, 1 \mathrm{H})$, $7.54(\mathrm{dd}, J=7.9,1.8 \mathrm{~Hz}, 1 \mathrm{H}), 7.27(\mathrm{dd}, J=8.9,1.7 \mathrm{~Hz}, 1 \mathrm{H}), 7.09(\mathrm{~d}, J=8.3 \mathrm{~Hz}, 1 \mathrm{H}), 7.01(\mathrm{t}, J$ $=7.5 \mathrm{~Hz}, 1 \mathrm{H}) .{ }^{13} \mathrm{C}$ NMR $\left(125 \mathrm{MHz}\right.$, Acetone- $\left.d_{6}\right) \delta 200.39,162.71,160.01,140.29,135.91$, $132.25,128.81,127.11,123.52,121.41,119.55,117.82,117.48$. HRMS (ESI) m/z Calcd for $\left[\mathrm{C}_{13} \mathrm{H}_{9} \mathrm{NNaO}_{5}, \mathrm{M}+\mathrm{Na}\right]^{+}: 282.0373$; Found 282.0376 .
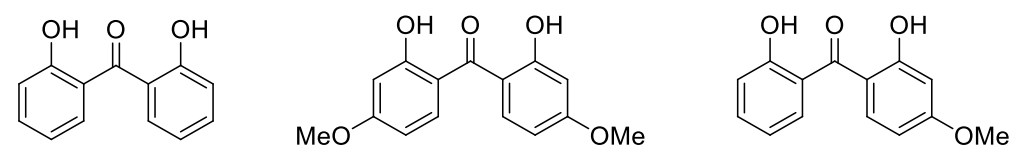

These three compounds are commercial available

2.3. General procedure C: Synthesis of $N$-methoxy- $N$-methyl-2-(tolunesulfonamide) benzamide: 


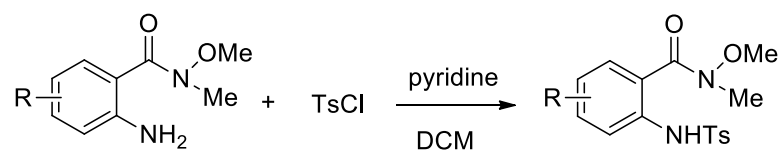

To a solution of anthranilic acid $N$-methoxy- $N$-methylamide derivative $(2.8 \mathrm{mmol})$ in dichloromethane was added pyridine $(0.66 \mathrm{~mL}, 8.4 \mathrm{mmoL})$ and $p$-toluenesulfonyl chloride $(1.06 \mathrm{~g}, 5.6 \mathrm{mmol})$ dropwise under $0{ }^{\circ} \mathrm{C}$. The mixture was then stirred at room temperature for 2 days. Dilute the reaction with equal amount of water. The resulting mixture was extracted with dichloromethane $(3 \times 10 \mathrm{~mL})$, and the combined extracts were washed with brine and dried over sodium sulphate. The crude was purified by chromatography on silica gel (3:1 hexane/ethyl acetate), and gave the title compound.

\subsection{General procedure D: Synthesis of $N$-(2-(2-methoxybenzoyl)phenyl)-} toluenesulfonamide derivative:

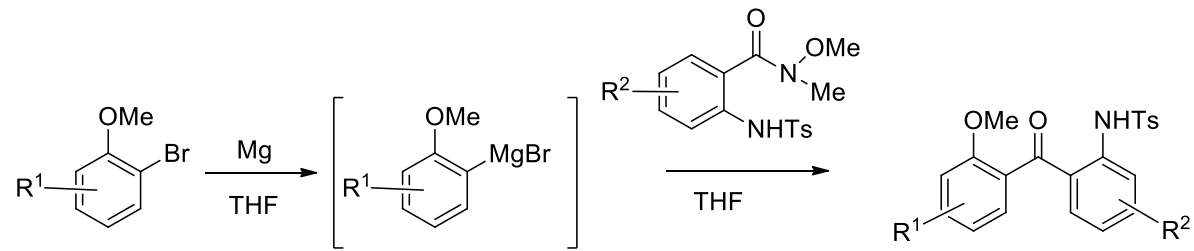

Synthesis of Grignard reagent: To dry, $\mathrm{N}_{2}$-flushed $100 \mathrm{ml}$ two-necked, round-bottom flask, equipped with a magnetic stir bar and a reflux condenser, was charged with $\mathrm{Mg}$ turning $(0.22 \mathrm{~g}, 10 \mathrm{mmol})$ and THF $(20 \mathrm{~mL})$ at room temperature. The contents were added a small crystal of iodine, the solvation of iodine crystal formed a brown solution. To a second round-bottom-flask was added bromoanisole $(7.5 \mathrm{mmol})$ along with THF $(5 \mathrm{~mL})$. The bromide precursor was then added to the first flask in dropwise manner, and the contents were stirred until the Grignard reaction commenced. The remainder of bromoanisole was added over $30 \mathrm{~min}$ while maintaining a steady exotherm. The mixture was warmed to reflux at 75 ${ }^{\circ} \mathrm{C}$ for $2 \mathrm{~h}$. The Grignard reagent was used for next step directly.

Under $\mathrm{N}_{2}$-protection, to a mixture of $\mathrm{N}$-methoxy- $\mathrm{N}$-methyl-2-(tolunesulfonamide) benzamide $(2.5 \mathrm{mmol})$ dissolved in $\operatorname{THF}(10 \mathrm{~mL})$ was added Grignard reagent at $0{ }^{\circ} \mathrm{C}$ and continue stirring for $1 \mathrm{~h}$ at room temperature, the reaction progress was monitored by TLC. The reaction was quenched with ammonium chloride solution $(15 \mathrm{~mL})$. The resulting mixture was extracted with ethyl acetate $(3 \times 10 \mathrm{~mL})$, and the combined extracts were washed with 
brine and dried over sodium sulphate. The crude was purified by chromatography on silica gel (10:1 hexane/ethyl acetate), and gave the title compound.

\subsection{General procedure E: Synthesis of $N$-(2-(2-hydroxybenzoyl)phenyl)-} toluenesulfonamide derivatives

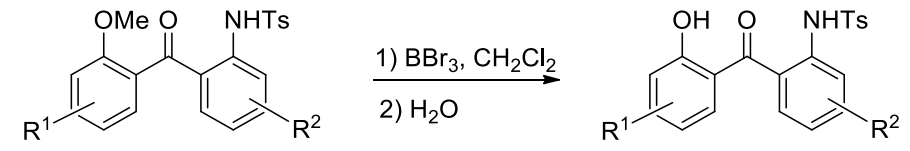

$\begin{array}{llllllll}\text { (Reaction was proceed under } & -78 & \left.{ }^{\circ} \mathrm{C}\right) & \text { To } & \text { a } & \text { solution of }\end{array}$ $N$-(2-(2-methoxybenzoyl)phenyl)-toluenesulfonamide derivative $(1.3 \mathrm{mmol})$ in dichloromethane was added boron tribromide $(1.3 \mathrm{M}, 3.0 \mathrm{~mL}, 3.9 \mathrm{mmol})$ in dropwise manner. The mixture was then stirred under room temperature overnight. The reaction was quenched and neutralized with saturated sodium bicarbonate solution to $\sim \mathrm{pH} 7$, followed by addition of water to separate the dichloromethane layer. The collected aqueous layer then extracted with ethyl acetate $(3 \times 10 \mathrm{~mL})$, and the combined extracts were washed with brine and dried over sodium sulphate. The crude was purified by chromatography on silica gel (10:1 hexane/ethyl acetate), and gave the title compound.<smiles>COc1ccccc1C(=O)c1ccccc1N(C)S(=O)(=O)c1ccccc1</smiles>

Following the general procedure D. used the $S$-1. $80 \%$ yield. White solid, MP: $132-134{ }^{\circ} \mathrm{C}$. ${ }^{1} \mathbf{H}$ NMR (500 MHz, $\left.\mathrm{CDCl}_{3}\right): \delta 11.14(\mathrm{~s}, 1 \mathrm{H}), 7.78$ - $7.75(\mathrm{~m}, 3 \mathrm{H}), 7.49$ - $7.44(\mathrm{~m}, 2 \mathrm{H}), 7.34$ (d, $J=8.0 \mathrm{~Hz}, 1 \mathrm{H}), 7.23(\mathrm{~d}, J=8.0 \mathrm{~Hz}, 2 \mathrm{H}), 7.07-6.96(\mathrm{~m}, 4 \mathrm{H}), 3.67(\mathrm{~s}, 3 \mathrm{H}), 2.38(\mathrm{~s}, 3 \mathrm{H})$. ${ }^{13}$ C NMR (125 MHz, $\left.\mathrm{CDCl}_{3}\right): \delta 199.84,156.89,143.71,140.05,136.70,134.57,134.51$, 132.20, 129.61, 129.19, 128.47, 127.38, 124.07, 122.59, 120.39, 119.82, 111.47, 55.56, 21.51. HRMS (ESI) m/z Calcd for $\left[\mathrm{C}_{21} \mathrm{H}_{29} \mathrm{NNaO}_{4} \mathrm{~S}, \mathrm{M}+\mathrm{Na}\right]^{+}$: 404.0927; Found 404.0925.<smiles>CN(c1ccccc1)c1ccccc1C(=O)c1ccccc1</smiles>

Following the general procedure E. $\quad 75 \%$ yield. Yellow solid, MP: $151-152{ }^{\circ} \mathrm{C} .{ }^{1} \mathbf{H}$ NMR $\left(500 \mathrm{MHz}, \mathrm{CDCl}_{3}\right): \delta 11.53(\mathrm{~s}, 1 \mathrm{H}), 8.53(\mathrm{~s}, 1 \mathrm{H}), 7.80(\mathrm{~d}, J=8.0 \mathrm{~Hz}, 1 \mathrm{H}), 7.57(\mathrm{t}, J=8.0 \mathrm{~Hz}$, 
1H), $7.49(\mathrm{td}, J=8.01 .0 \mathrm{~Hz}, 1 \mathrm{H}), 7.42(\mathrm{~d}, J=7.5 \mathrm{~Hz}, 2 \mathrm{H}), 7.31(\mathrm{~d}, J=7.5 \mathrm{~Hz}, 1 \mathrm{H}), 7.25(\mathrm{t}$, $J=7.5 \mathrm{~Hz}, 1 \mathrm{H}), 7.01(\mathrm{~d}, J=8.0 \mathrm{~Hz}, 1 \mathrm{H}), 6.90(\mathrm{~d}, J=7.5 \mathrm{~Hz}, 2 \mathrm{H}), 6.86(\mathrm{~d}, J=8.0 \mathrm{~Hz}, 1 \mathrm{H})$, $6.71(\mathrm{t}, J=7.5 \mathrm{~Hz}, 1 \mathrm{H}), 2.08(\mathrm{~s}, 3 \mathrm{H}) .{ }^{13} \mathbf{C}$ NMR $\left(125 \mathrm{MHz}, \mathrm{CDCl}_{3}\right): \delta 200.89,163.39,143.72$, $136.73,136.52$, 135.27, 133.61, 132.94, 131.24, 129.58, 129.42, 126.96, 126.69, 124.66, 118.77, 118.42, 118.28, 21.29. HRMS (ESI) $\mathrm{m} / \mathrm{z}$ Calcd for $\left[\mathrm{C}_{20} \mathrm{H}_{17} \mathrm{NNaO}_{4} \mathrm{~S}, \mathrm{M}+\mathrm{Na}\right]^{+}$: 390.0770; Found 390.0774.

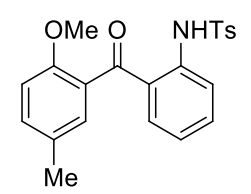

Following the general procedure D. $85 \%$ yield. White solid, MP: $137-138{ }^{\circ} \mathrm{C} .{ }^{1} \mathbf{H}$ NMR $\left(500 \mathrm{MHz}, \mathrm{CDCl}_{3}\right): \delta 11.15(\mathrm{~s}, 1 \mathrm{H}), 7.78-7.75(\mathrm{~m}, 3 \mathrm{H}), 7.46(\mathrm{t}, J=8.0 \mathrm{~Hz}, 1 \mathrm{H}), 7.36(\mathrm{~d}, J=$ $8.0 \mathrm{~Hz}, 1 \mathrm{H}), 7.27-7.22(\mathrm{~m}, 3 \mathrm{H}), 6.98(\mathrm{t}, J=8.0 \mathrm{~Hz}, 1 \mathrm{H}), 6.87-6.85(\mathrm{~m}, 2 \mathrm{H}), 3.62(\mathrm{~s}, 3 \mathrm{H})$, $2.38(\mathrm{~s}, 3 \mathrm{H}), 2.30(\mathrm{~s}, 3 \mathrm{H}) .{ }^{13} \mathbf{C}$ NMR $\left(125 \mathrm{MHz}, \mathrm{CDCl}_{3}\right): \delta 200.09,154.82,143.68,139.97$, 136.72 , 134.60, 134.52, 132.61, 129.84, 129.60, 129.48, 128.28, 127.38, 124.14, 122.63, 119.83, 111.49, 55.68, 21.52, 20.33. HRMS (ESI) m/z Calcd for $\left[\mathrm{C}_{22} \mathrm{H}_{21} \mathrm{NNaO}_{4} \mathrm{~S}, \mathrm{M}+\mathrm{Na}\right]^{+}$: 418.1083; Found 418.1088.

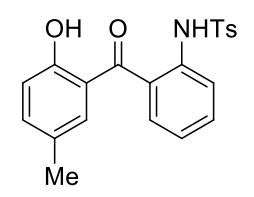

Following the general procedure E. $80 \%$ yield. Yellow solid, MP: $105-106{ }^{\circ} \mathrm{C} .{ }^{\mathbf{1}} \mathbf{H}$ NMR $\left(500 \mathrm{MHz}, \mathrm{CDCl}_{3}\right): \delta 11.36(\mathrm{~s}, 1 \mathrm{H}), 8.50(\mathrm{~s}, 1 \mathrm{H}), 7.79(\mathrm{~d}, J=8.0 \mathrm{~Hz}, 1 \mathrm{H}), 7.57(\mathrm{td}, J=8.5$ $3.5 \mathrm{~Hz}, 1 \mathrm{H}), 7.42(\mathrm{~d}, J=8.0 \mathrm{~Hz}, 2 \mathrm{H}), 7.31-7.29$ (m, $2 \mathrm{H}), 7.26(\mathrm{t}, J=8.0 \mathrm{~Hz}, 1 \mathrm{H}), 6.92-$ $6.89(\mathrm{~m}, 3 \mathrm{H}), 6.60(\mathrm{~d}, J=2.0 \mathrm{~Hz}, 1 \mathrm{H}), 2.19(\mathrm{~s}, 3 \mathrm{H}), 2.07(\mathrm{~s}, 3 \mathrm{H}) .{ }^{13} \mathbf{C}$ NMR $(125 \mathrm{MHz}$, $\left.\mathrm{CDCl}_{3}\right): \delta 200.78,161.38,143.58,137.85,136.36,135.38,133.23,132.80,131.07,129.70$, 129.39, 127.27, 127.05, 126.86, 124.70, 118.45, 118.16, 21.17, 20.41. HRMS (ESI) m/z Calcd for $\left[\mathrm{C}_{21} \mathrm{H}_{19} \mathrm{NNaO}_{4} \mathrm{~S}, \mathrm{M}+\mathrm{Na}\right]^{+}$: 404.1034; Found 404.1038.

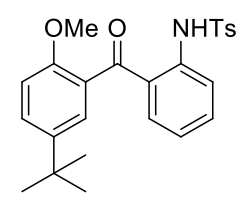


Following the general procedure D. 78\% yield. White solid, MP: $68-70{ }^{\circ} \mathrm{C} .{ }^{1} \mathbf{H}$ NMR (500 $\left.\mathrm{MHz}, \mathrm{CDCl}_{3}\right): \delta 11.27(\mathrm{~s}, 1 \mathrm{H}), 7.80-7.75(\mathrm{~m}, 3 \mathrm{H}), 7.49-7.43(\mathrm{~m}, 2 \mathrm{H}), 7.37$ (d, J = 7.0 Hz, 1H), 7.24 (d, $J=7.5 \mathrm{~Hz}, 2 \mathrm{H}), 7.17$ (s, 1H). 6.97 (t, $J=7.5 \mathrm{~Hz}, 1 \mathrm{H}), 6.90$ (d, $J=8.0 \mathrm{~Hz}, 1 \mathrm{H})$, $3.62(\mathrm{~s}, 3 \mathrm{H}), 2.37$ (s, 3H), 1.30 (s, 9H). $\left.{ }^{13} \mathbf{C ~ N M R ~ ( 1 2 5 ~ M H z , ~} \mathrm{CDCl}_{3}\right): \delta 200.52,154.64$, $143.70,143.40,140.09,136.79,134.76,134.56,129.62$, 129.12, 127.88, 127.38, 126.07, 123.86, 122.47, 119.40, 111.14, 55.61, 34.18, 31.38, 21.54. HRMS (ESI) m/z Calcd for $\left[\mathrm{C}_{25} \mathrm{H}_{27} \mathrm{NNaO}_{4} \mathrm{~S}, \mathrm{M}+\mathrm{Na}\right]^{+}$: 437.1660; Found 437.1664.

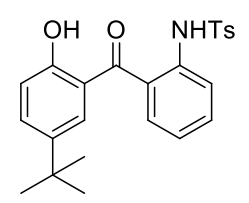

Following the general procedure E. $80 \%$ yield. Yellow solid, MP: $178-179{ }^{\circ} \mathrm{C} .{ }^{1} \mathbf{H}$ NMR $\left(500 \mathrm{MHz}, \mathrm{CDCl}_{3}\right): \delta 11.37(\mathrm{~s}, 1 \mathrm{H}), 8.77(\mathrm{~s}, 1 \mathrm{H}), 7.80(\mathrm{~d}, J=8.5 \mathrm{~Hz}, 1 \mathrm{H}), 7.59-7.52(\mathrm{~m}, 4 \mathrm{H})$, $7.36(\mathrm{dd}, J=7.51 .0 \mathrm{~Hz}, 1 \mathrm{H}), 7.24(\mathrm{t}, J=7.5 \mathrm{~Hz}, 1 \mathrm{H}), 7.22(\mathrm{~s}, 1 \mathrm{H}), 7.04-6.96(\mathrm{~m}, 3 \mathrm{H}), 2.10$ (s, 3H), 1.23 (s, 9H). ${ }^{13} \mathbf{C}$ NMR (125 MHz, $\left.\mathrm{CDCl}_{3}\right): \delta 200.89,161.29,143.49,140.93,136.97$, $135.72,134.56,133.10,131.56,129.71,129.57,128.52,127.01,125.88,124.06,118.04$, 118.01, 34.00, 31.19, 21.36. HRMS (ESI) $\mathrm{m} / \mathrm{z}$ Calcd for $\left[\mathrm{C}_{24} \mathrm{H}_{25} \mathrm{NNaO}_{4} \mathrm{~S}, \mathrm{M}+\mathrm{Na}\right]^{+}$: 446.1396; Found 446.1395.

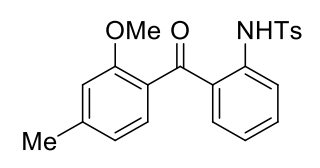

Following the general procedure D. $88 \%$ yield. White solid, MP: $120-121{ }^{\circ}$ C. ${ }^{1} \mathbf{H}$ NMR (500 MHz, $\left.\mathrm{CDCl}_{3}\right): \delta 11.04$ (s, 1H), $7.76-7.74$ (m, $\left.3 \mathrm{H}\right), 7.44$ (t, $\left.J=8.0 \mathrm{~Hz}, 1 \mathrm{H}\right), 7.35$ (d, $J=$ $8.0 \mathrm{~Hz}, 1 \mathrm{H}), 7.21(\mathrm{~d}, J=8.0 \mathrm{~Hz}, 2 \mathrm{H}), 6.98-6.95(\mathrm{~m}, 2 \mathrm{H}), 6.81(\mathrm{~d}, J=7.5 \mathrm{~Hz}, 1 \mathrm{H}), 6.78(\mathrm{~s}$, 1H), 3.65 (s, 3H), 2.42 (s, 3H), 2.36 (s, 3H). $\left.{ }^{13} \mathbf{C ~ N M R ~ ( 1 2 5 ~ M H z , ~} \mathrm{CDCl}_{3}\right): \delta$ 199.62, 157.21, $143.62,143.26,139.83,136.73,134.34,134.25,129.57,129.55,127.36,125.68,124.73$, 122.60, 121.04, 120.10, 112.30, 55.49, 21.89, 21.48. HRMS (ESI) m/z Calcd for $\left[\mathrm{C}_{22} \mathrm{H}_{21} \mathrm{NNaO}_{4} \mathrm{~S}, \mathrm{M}+\mathrm{Na}\right]^{+}:$418.1083; Found 418.1082.<smiles></smiles> 
Following the general procedure E. $80 \%$ yield. Yellow solid, MP: $133-134{ }^{\circ} \mathrm{C} .{ }^{1} \mathbf{H}$ NMR $\left(500 \mathrm{MHz}, \mathrm{CDCl}_{3}\right): \delta 11.64(\mathrm{~s}, 1 \mathrm{H}), 8.50(\mathrm{~s}, 1 \mathrm{H}), 7.78(\mathrm{~d}, J=8.0 \mathrm{~Hz}, 1 \mathrm{H}), 7.55(\mathrm{t}, J=8.0 \mathrm{~Hz}$, 1H), $7.41(\mathrm{~d}, J=7.5 \mathrm{~Hz}, 2 \mathrm{H}), 7.29(\mathrm{~d}, J=8.0 \mathrm{~Hz}, 1 \mathrm{H}), 7.23$ (t, $J=7.5 \mathrm{~Hz}, 1 \mathrm{H}), 6.89$ (d, $J=$ $8.0 \mathrm{~Hz}, 1 \mathrm{H}), 6.82(\mathrm{~s}, 1 \mathrm{H}), 6.75(\mathrm{~d}, J=8.0 \mathrm{~Hz}, 1 \mathrm{H}), 6.52(\mathrm{~d}, J=8.0 \mathrm{~Hz}, 1 \mathrm{H}), 2.38(\mathrm{~s}, 3 \mathrm{H}), 2.07$ (s, 3H). ${ }^{13}$ C NMR (125 MHz, $\left.\mathrm{CDCl}_{3}\right): \delta 200.17,163.19,148.75,143.67,136.36,135.37$, $133.55,132.66,131.02,129.64,129.50,126.93,126.67$, 124.62, 119.65, 118.42, 116.58, 21.99, 21.21. HRMS (ESI) $\mathrm{m} / \mathrm{z}$ Calcd for $\left[\mathrm{C}_{21} \mathrm{H}_{19} \mathrm{NNaO}_{4} \mathrm{~S}, \mathrm{M}+\mathrm{Na}\right]^{+}:$404.1034; Found 404.1032 .

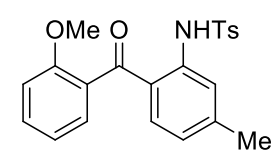

Following the general procedure D. $85 \%$ yield. White solid, MP: $109-110{ }^{\circ}$ C. ${ }^{1} \mathbf{H}$ NMR $\left(500 \mathrm{MHz}, \mathrm{CDCl}_{3}\right): \delta 11.26(\mathrm{~s}, 1 \mathrm{H}), 7.78(\mathrm{~d}, J=8.0 \mathrm{~Hz}, 2 \mathrm{H}), 7.57(\mathrm{~s}, 1 \mathrm{H}), 7.45(\mathrm{td}, J=7.0$ $1.5 \mathrm{~Hz}, 1 \mathrm{H}), 7.24-7.21(\mathrm{~m}, 3 \mathrm{H}), 7.04(\mathrm{dd}, J=7.02 .0 \mathrm{~Hz}, 1 \mathrm{H}), 7.00(\mathrm{~d}, J=8.0 \mathrm{~Hz}, 1 \mathrm{H}), 6.97(\mathrm{~d}$, $J=8.0 \mathrm{~Hz}, 1 \mathrm{H}), 6.77(\mathrm{~d}, J=7.0 \mathrm{~Hz}, 1 \mathrm{H}), 3.68(\mathrm{~s}, 3 \mathrm{H}), 2.38(\mathrm{~s}, 3 \mathrm{H}), 2.36(\mathrm{~s}, 3 \mathrm{H}),{ }^{13} \mathbf{C} \mathbf{~ N M R}$ $\left(125 \mathrm{MHz}, \mathrm{CDCl}_{3}\right): \delta 199.48,156.74,146.15,143.65,140.28,136.78,134.69,131.92,129.58$, $129.01,128.72,127.36,123.58,121.57,120.35,120.10,111.45,55.58,22.10,21.50$. HRMS (ESI) $\mathrm{m} / \mathrm{z}$ Calcd for $\left[\mathrm{C}_{22} \mathrm{H}_{21} \mathrm{NNaO}_{4} \mathrm{~S}, \mathrm{M}+\mathrm{Na}\right]^{+}$: 418.1083; Found 418.1080.

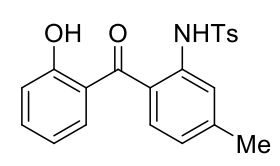

Following the general procedure E. $80 \%$ yield. Yellow solid, MP: $161-162{ }^{\circ} \mathrm{C} .{ }^{1} \mathbf{H}$ NMR $\left(500 \mathrm{MHz}, \mathrm{CDCl}_{3}\right): \delta 11.49(\mathrm{~s}, 1 \mathrm{H}), 8.65(\mathrm{~s}, 1 \mathrm{H}), 7.61(\mathrm{~s}, 1 \mathrm{H}), 7.47(\mathrm{t}, J=8.0 \mathrm{~Hz}, 1 \mathrm{H}), 7.42(\mathrm{~d}$, $J=7.5 \mathrm{~Hz}, 2 \mathrm{H}), 7.21(\mathrm{~d}, J=8.0 \mathrm{~Hz}, 1 \mathrm{H}), 7.04(\mathrm{~d}, J=7.5 \mathrm{~Hz}, 1 \mathrm{H}), 7.00(\mathrm{~d}, J=8.0 \mathrm{~Hz}, 1 \mathrm{H})$, $6.90-6.86(\mathrm{~m}, 3 \mathrm{H}), 6.71(\mathrm{~d}, J=7.5 \mathrm{~Hz}, 1 \mathrm{H}), 2.46(\mathrm{~s}, 3 \mathrm{H}), 2.07(\mathrm{~s}, 3 \mathrm{H}) .{ }^{13} \mathbf{C}$ NMR $(125 \mathrm{MHz}$, $\left.\mathrm{CDCl}_{3}\right): \delta 200.82,163.21,144.25,143.65,136.78,136.47,135.28,133.57,131.49,129.54$, 127.06, 126.98, 126.62, 125.47, 118.92, 118.35, 118.16, 21.76, 21.27. HRMS (ESI) m/z Calcd for $\left[\mathrm{C}_{21} \mathrm{H}_{19} \mathrm{NNaO}_{4} \mathrm{~S}, \mathrm{M}+\mathrm{Na}\right]^{+}$: 404.1034; Found 404.1036.

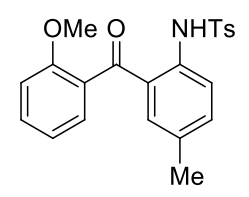


Following the general procedure D. $90 \%$ yield. White solid, MP: $109-110{ }^{\circ} \mathrm{C}$. ${ }^{1} \mathbf{H}$ NMR $\left(500 \mathrm{MHz}, \mathrm{CDCl}_{3}\right): \delta 10.88(\mathrm{~s}, 1 \mathrm{H}), 7.72(\mathrm{~d}, J=8.0 \mathrm{~Hz}, 2 \mathrm{H}), 7.67(\mathrm{~d}, J=8.5 \mathrm{~Hz}, 1 \mathrm{H}), 7.49-$ $7.46(\mathrm{~m}, 1 \mathrm{H}), 7.28(\mathrm{dd}, J=8.51 .5 \mathrm{~Hz}, 1 \mathrm{H}), 7.20(\mathrm{~d}, J=8.0 \mathrm{~Hz}, 1 \mathrm{H}), 7.09(\mathrm{~d}, J=1.0 \mathrm{~Hz}, 1$ H), $7.00-7.69$ (m, 3H), 3.68 (s, 3H), 2.36 (s, 3H), 2.18 (s, 3H). $\left.{ }^{13} \mathbf{C ~ N M R ~ ( 1 2 5 ~ M H z , ~} \mathrm{CDCl}_{3}\right)$ : $\delta 199.69,157.00,143.54,137.47,136.67,135.34,134.41,132.51,132.17,129.54,129.27$, 128.48, 127.37, 124.61, 120.59, 120.27, 111.57, 55.61, 21.48, 20.56. HRMS (ESI) m/z Calcd for $\left[\mathrm{C}_{22} \mathrm{H}_{21} \mathrm{NNaO}_{4} \mathrm{~S}, \mathrm{M}+\mathrm{Na}\right]^{+}:$418.1083; Found 418.1080.<smiles>Nc1ccc([N+](=O)[O-])c(C(=O)c2ccccc2O)c1</smiles>

Following the general procedure E. $82 \%$ yield. Yellow solid, MP: $112-113{ }^{\circ} \mathrm{C} .{ }^{1} \mathbf{H}$ NMR $\left(500 \mathrm{MHz}, \mathrm{CDCl}_{3}\right): \delta 11.57(\mathrm{~s}, 1 \mathrm{H}), 8.31(\mathrm{~s}, 1 \mathrm{H}), 7.68(\mathrm{~d}, J=8.5 \mathrm{~Hz}, 1 \mathrm{H}), 7.49$ (t, $J=8.5 \mathrm{~Hz}$, $1 \mathrm{H}), 7.38(\mathrm{~d}, J=8.5 \mathrm{~Hz}, 2 \mathrm{H}), 7.08(\mathrm{~s}, 1 \mathrm{H}), 7.00(\mathrm{~d}, J=8.0 \mathrm{~Hz}, 1 \mathrm{H}), 6.87-7.83(\mathrm{~m}, 3 \mathrm{H})$, $6.71(\mathrm{t}, J=7.5 \mathrm{~Hz}, 1 \mathrm{H}), 2.35(\mathrm{~s}, 3 \mathrm{H}), 2.05$ (s, 3H). ${ }^{13} \mathbf{C} \mathbf{N M R}\left(125 \mathrm{MHz}, \mathrm{CDCl}_{3}\right): \delta 200.95$, $163.39,143.56,136.60,135.21,134.91,133.70,133.64,133.59,131.37,129.90,129.53$, 127.32, 126.97, 118.71, 118.36, 118.22, 21.28, 20.90. HRMS (ESI) m/z Calcd for $\left[\mathrm{C}_{21} \mathrm{H}_{19} \mathrm{NNaO}_{4} \mathrm{~S}, \mathrm{M}+\mathrm{Na}\right]^{+}:$404.1034; Found 404.1032.

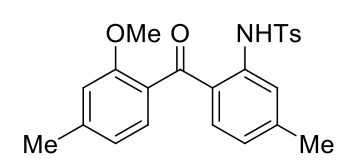

Following the general procedure D. $86 \%$ yield. White solid, MP: $70-71{ }^{\circ} \mathrm{C} .{ }^{1} \mathbf{H}$ NMR $(500$ $\left.\mathrm{MHz}, \mathrm{CDCl}_{3}\right): \delta 11.19(\mathrm{~s}, 1 \mathrm{H}), 7.76(\mathrm{~d}, J=8.0 \mathrm{~Hz}, 2 \mathrm{H}), 7.57(\mathrm{~s}, 1 \mathrm{H}), 7.24-7.21(\mathrm{~m}, 3 \mathrm{H})$, $6.93(\mathrm{~d}, J=8.0 \mathrm{~Hz}, 1 \mathrm{H}), 6.80-6.75(\mathrm{~m}, 3 \mathrm{H}), 3.66(\mathrm{~s}, 3 \mathrm{H}), 2.42(\mathrm{~s}, 3 \mathrm{H}), 2.37$ (s, 3H), $2.36(\mathrm{~s}$, 3H). ${ }^{13}$ C NMR (125 MHz, $\left.\mathrm{CDCl}_{3}\right): \delta 199.34,157.02,145.78,143.57,142.88,140.08,136.79$, $134.57,129.54,129.36,127.33,125.93,123.56,122.12,120.98,120.35,112.28,55.52,22.04$, 21.86, 21.48. HRMS (ESI) $\mathrm{m} / \mathrm{z}$ Calcd for $\left[\mathrm{C}_{23} \mathrm{H}_{23} \mathrm{NNaO}_{4} \mathrm{~S}, \mathrm{M}+\mathrm{Na}\right]^{+}$: 432.1240; Found 432.1244.<smiles></smiles> 
Following the general procedure E. $81 \%$ yield. Yellow solid, MP: $135-136{ }^{\circ} \mathrm{C} .{ }^{1} \mathbf{H}$ NMR $\left(500 \mathrm{MHz}, \mathrm{CDCl}_{3}\right): \delta 11.62(\mathrm{~s}, 1 \mathrm{H}), 8.62(\mathrm{~s}, 1 \mathrm{H}), 7.60(\mathrm{~s}, 1 \mathrm{H}), 7.42 \quad(\mathrm{~d}, J=7.0 \mathrm{~Hz}, 2 \mathrm{H})$, $7.19(\mathrm{~d}, J=7.5 \mathrm{~Hz}, 1 \mathrm{H}), 7.03(\mathrm{~d}, J=8.0 \mathrm{~Hz}, 1 \mathrm{H}), 6.90(\mathrm{~d}, J=7.0 \mathrm{~Hz}, 2 \mathrm{H}), 6.81(\mathrm{~s}, 1 \mathrm{H}), 6.77$ $(\mathrm{d}, J=8.0 \mathrm{~Hz}, 1 \mathrm{H}), 6.52(\mathrm{~d}, J=8.0 \mathrm{~Hz}, 1 \mathrm{H}), 2.45(\mathrm{~s}, 3 \mathrm{H}), 2.37(\mathrm{~s}, 3 \mathrm{H}), 2.06(\mathrm{~s}, 3 \mathrm{H}),{ }^{13} \mathrm{C}$ NMR (125 MHz, $\left.\mathrm{CDCl}_{3}\right): \delta 200.15,163.52,148.41,143.89,143.58,136.59,135.37,133.52$, 131.23, 129.46, 127.08, 126.95, 126.85, 125.43, 119.50, 118.36, 116.70, 21.95, 21.71, 21.19.

HRMS (ESI) m/z Calcd for $\left[\mathrm{C}_{22} \mathrm{H}_{21} \mathrm{NNaO}_{4} \mathrm{~S}, \mathrm{M}+\mathrm{Na}\right]^{+}:$418.1083; Found 418.1082.

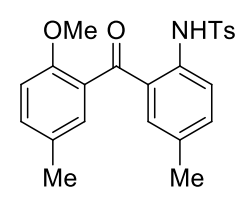

Following the general procedure D. $82 \%$ yield. White solid, MP: $95-96{ }^{\circ} \mathrm{C}$. ${ }^{1} \mathbf{H}$ NMR (500 $\left.\mathrm{MHz}, \mathrm{CDCl}_{3}\right): \delta 10.90(\mathrm{~s}, 1 \mathrm{H}), 7.73(\mathrm{~d}, J=8.0 \mathrm{~Hz}, 2 \mathrm{H}), 7.67(\mathrm{t}, J=7.5 \mathrm{~Hz}, 1 \mathrm{H}), 7.27-7.25$ (m, 2H), $7.21(\mathrm{~d}, J=8.0 \mathrm{~Hz}, 2 \mathrm{H}), 7.11(\mathrm{~s}, 1 \mathrm{H}), 6.87$ (d, J = 8.5 Hz, 1H), $6.80(\mathrm{~s}, 1 \mathrm{H}), 3.63$ (s, 3H), 2.37 (s, 3H), 2.30 (s, 3H), 2.19 (s, 3H). ${ }^{13} \mathbf{C}$ NMR (125 MHz, $\mathrm{CDCl}_{3}$ ): $\delta$ 199.97, 154.89, $143.47,137.39,136.73,135.28,134.48,132.56,132.49,129.75,129.52,129.50,128.36$, 127.37, 124.62, 120.54, 111.56, 55.71, 21.50, 20.57, 20.34. HRMS (ESI) m/z Calcd for $\left[\mathrm{C}_{23} \mathrm{H}_{23} \mathrm{NNaO}_{4} \mathrm{~S}, \mathrm{M}+\mathrm{Na}\right]^{+}:$432.1240; Found 432.1245.

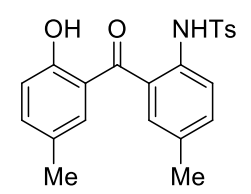

Following the general procedure E. 82\% yield. Yellow solid, MP: $142-143{ }^{\circ} \mathrm{C} .{ }^{1} \mathbf{H}$ NMR $\left(500 \mathrm{MHz}, \mathrm{CDCl}_{3}\right): \delta 11.41(\mathrm{~s}, 1 \mathrm{H}), 8.27(\mathrm{~s}, 1 \mathrm{H}), 7.68(\mathrm{~d}, J=8.5 \mathrm{~Hz}, 1 \mathrm{H}), 7.40-7.38(\mathrm{~m}, 3 \mathrm{H})$, $7.30(\mathrm{dd}, J=8.51 .5 \mathrm{~Hz}, 1 \mathrm{H}), 7.08(\mathrm{~s}, 1 \mathrm{H}), 6.91-6.87(\mathrm{~m}, 3 \mathrm{H}), 6.57$ (s, 1H), $2.36(\mathrm{~s}, 3 \mathrm{H})$, 2.20 (s, 3H), 2.05 (s, 3H). ${ }^{13} \mathbf{C}$ NMR (125 MHz, $\left.\mathrm{CDCl}_{3}\right): \delta 200.85,161.38,143.41,137.74$, $135.33,134.92$, 133.52, 133.46, 133.25, 131.17, 130.18, 129.33, 127.47, 127.14, 127.07, 118.39, 118.09, 21.16, 20.93, 20.45. HRMS (ESI) m/z Calcd for $\left[\mathrm{C}_{22} \mathrm{H}_{21} \mathrm{NNaO}_{4} \mathrm{~S}, \mathrm{M}+\mathrm{Na}\right]^{+}$: 418.1083; Found 418.1086. 


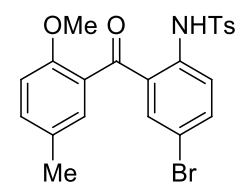

Following the general procedure D. used the known $S$-6 [ ]. 85\% yield. White solid, MP: 131 $-132{ }^{\circ} \mathrm{C} .{ }^{1} \mathbf{H}$ NMR $\left(500 \mathrm{MHz}, \mathrm{CDCl}_{3}\right): \delta 10.90(\mathrm{~s}, 1 \mathrm{H}), 7.74(\mathrm{~d}, J=8.0 \mathrm{~Hz}, 2 \mathrm{H}), 7.68(\mathrm{~d}, J=$ $9.0 \mathrm{~Hz}, 1 \mathrm{H}), 7.54(\mathrm{~d}, J=9.0 \mathrm{~Hz}, 1 \mathrm{H}), 7.44(\mathrm{~s}, 1 \mathrm{H}), 7.29$ (d, $J=8.5 \mathrm{~Hz}, 1 \mathrm{H}), 7.24$ (d, $J=7.5$ $\mathrm{Hz}, 2 \mathrm{H}), 6.92(\mathrm{~s}, 1 \mathrm{H}), 6.87$ (d, $J=8.5 \mathrm{~Hz}, 1 \mathrm{H}), 3.61(\mathrm{~s}, 3 \mathrm{H}), 2.38(\mathrm{~s}, 3 \mathrm{H}), 2.31$ (s, 3H). ${ }^{13} \mathrm{C}$ NMR (125 MHz, $\left.\mathrm{CDCl}_{3}\right): \delta$ 198.72, 154.95, 143.93, 138.75, 137.03, 136.49, 136.44, 133.36, 133.33, 130.18, 129.69, 127.42, 127.34, 125.94, 121.78, 115.36, 111.68, 55.66, 21.51, 20.31. HRMS (ESI) $\mathrm{m} / \mathrm{z}$ Calcd for $\left[\mathrm{C}_{22} \mathrm{H}_{20}{ }^{79} \mathrm{BrNNaO}_{4} \mathrm{~S}, \mathrm{M}+\mathrm{Na}\right]^{+}$: 496.0188; Found 496.0186; $\left[\mathrm{C}_{22} \mathrm{H}_{20}{ }^{81} \mathrm{BrNNaO}_{4} \mathrm{~S}, \mathrm{M}+\mathrm{Na}\right]^{+}:$498.0168; Found 498.0167.

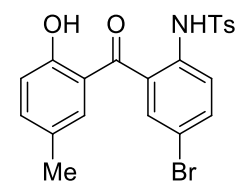

Following the general procedure E. $80 \%$ yield. Yellow solid, MP: $152-153{ }^{\circ} \mathrm{C} .{ }^{1} \mathbf{H}$ NMR (300 MHz, $\left.\mathrm{CDCl}_{3}\right): \delta 11.25(\mathrm{~s}, 1 \mathrm{H}), 8.30(\mathrm{~s}, 1 \mathrm{H}), 7.70(\mathrm{~d}, J=0.9 \mathrm{~Hz}, 2 \mathrm{H}), 7.45-7.42(\mathrm{~m}, 3 \mathrm{H})$, $7.33(\mathrm{td}, J=8.42 .1 \mathrm{~Hz}, 1 \mathrm{H}), 6.95-6.93(\mathrm{~m}, 3 \mathrm{H}), 6.58(\mathrm{~s}, 1 \mathrm{H}), 2.23(\mathrm{~s}, 3 \mathrm{H}), 2.10(\mathrm{~s}, 3 \mathrm{H}),{ }^{13} \mathrm{C}$ NMR (75 MHz, $\left.\mathrm{CDCl}_{3}\right): \delta 199.01,161.43,143.74,138.30,135.51,135.15,135.07,133.13$, $132.65,131.23,129.43,128.52,127.57,126.95,118.30,118.13,117.96,21.13,20.39$. HRMS (ESI) $\mathrm{m} / \mathrm{z}$ Calcd for $\left[\mathrm{C}_{21} \mathrm{H}_{18}{ }^{79} \mathrm{BrNNaO}_{4} \mathrm{~S}, \mathrm{M}+\mathrm{Na}\right]^{+}$: 482.0932; Found 482.0936; $\left[\mathrm{C}_{21} \mathrm{H}_{18}{ }^{81} \mathrm{BrNNaO} 4 \mathrm{~S}, \mathrm{M}+\mathrm{Na}\right]^{+}$: 484.0011; Found 484.0015.

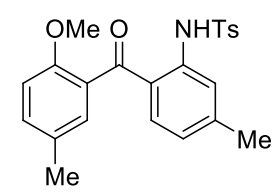

Following the general procedure D. $88 \%$ yield. White solid, MP: $144-145{ }^{\circ}$ C. ${ }^{1} \mathbf{H}$ NMR (500 MHz, $\left.\mathrm{CDCl}_{3}\right): \delta 11.27$ (s, 1H), 7.77 (d, $\left.J=8.5 \mathrm{~Hz}, 2 \mathrm{H}\right), 7.57$ (s, 1H), 7.25 - $7.22(\mathrm{~m}$, 4H), $6.86(\mathrm{~d}, J=8.5 \mathrm{~Hz}, 1 \mathrm{H}), 6.77(\mathrm{~d}, J=8.0 \mathrm{~Hz}, 1 \mathrm{H}), 3.64(\mathrm{~s}, 3 \mathrm{H}), 2.38(\mathrm{~s}, 3 \mathrm{H}), 2.36(\mathrm{~s}, 3 \mathrm{H})$, 2.29 (s, 3H). ${ }^{13} \mathrm{C}$ NMR (125 MHz, $\left.\mathrm{CDCl}_{3}\right): \delta$ 199.69, 154.67, 146.06, 143.60, 140.21, 136.81, 
$134.76,132.30,129.78,129.56,129.34,128.54,127.36,123.59,121.62,120.12,111.46$, 55.71, 22.10, 21.52, 20.32. $\left[\mathrm{C}_{23} \mathrm{H}_{23} \mathrm{NNaO}_{4} \mathrm{~S}, \mathrm{M}+\mathrm{Na}\right]^{+}$: 432.1240; Found 432.1244.<smiles>Cc1ccc(O)c(C(=O)c2ccc(C)cc2[N+](=O)[O-])c1</smiles>

Following the general procedure E. $80 \%$ yield. Yellow solid, MP: $111-112{ }^{\circ} \mathrm{C} .{ }^{1} \mathbf{H}$ NMR $\left(500 \mathrm{MHz}, \mathrm{CDCl}_{3}\right): \delta 11.33(\mathrm{~s}, 1 \mathrm{H}), 8.62(\mathrm{~s}, 1 \mathrm{H}), 7.61(\mathrm{~s}, 1 \mathrm{H}), 7.43 \quad(\mathrm{~d}, J=8.0 \mathrm{~Hz}, 2 \mathrm{H})$, $7.29(\mathrm{~d}, J=8.0 \mathrm{~Hz}, 1 \mathrm{H}), 7.20(\mathrm{~d}, J=8.0 \mathrm{~Hz}, 1 \mathrm{H}), 7.06(\mathrm{~d}, J=8.0 \mathrm{~Hz}, 1 \mathrm{H}), 6.91-6.90(\mathrm{~m}$, 3H), $6.61(\mathrm{~s}, 1 \mathrm{H}), 2.47(\mathrm{~s}, 3 \mathrm{H}), 2.20(\mathrm{~s}, 3 \mathrm{H}), 2.07(\mathrm{~s}, 3 \mathrm{H}),{ }^{13} \mathrm{C}$ NMR $\left(125 \mathrm{MHz}, \mathrm{CDCl}_{3}\right): \delta$ $200.73,161.19,144.06,143.51,137.55,136.61,135.37,133.23,131.30,129.35,127.26$, 127.13, 127.08, 126.90, 125.52, 118.61, 118.09, 21.76, 21.16, 20.42. HRMS (ESI) m/z Calcd for $\left[\mathrm{C}_{22} \mathrm{H}_{21} \mathrm{NNaO}_{4} \mathrm{~S}, \mathrm{M}+\mathrm{Na}\right]^{+}$: 418.1083; Found 418.1087.

\subsection{General procedure F: the synthesis of triols or diols.}

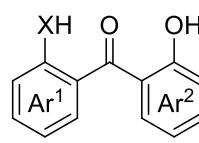

$\mathrm{X}=\mathrm{O}$ or NTs

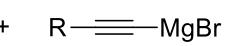

$\mathrm{R}=\mathrm{H}$ or $\mathrm{Me}$

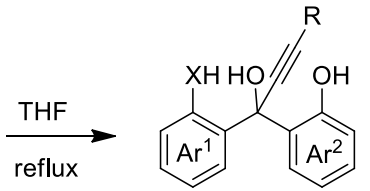

The Grignard reagent $(0.5 \mathrm{M}$ in THF, $20 \mathrm{~mL})$ was added slowly over a period of 10 minutes to a solution of ketone $(2.0 \mathrm{mmol})$ in dry THF $(20 \mathrm{~mL})$ at $0{ }^{\circ} \mathrm{C}$. Once addition was completed, the reaction mixture was allowed to stir at $70{ }^{\circ} \mathrm{C}$ for $3 \mathrm{~h}$, followed by the careful addition of saturated $\mathrm{NH}_{4} \mathrm{Cl}$ solution $(10 \mathrm{~mL})$. The biphasic mixture was extracted with ethyl acetate $(3 \mathrm{x}$ $30 \mathrm{~mL})$. The combined organic layer was washed with brine $(50 \mathrm{~mL})$, dried over $\mathrm{Na}_{2} \mathrm{SO}_{4}$ and concentrated in vacuo. Purification by column chromatography on silica gel with a mixture of hexanes and ethyl acetate $(10: 1 \mathrm{v} / \mathrm{v})$ afforded the desired product in a pure form.

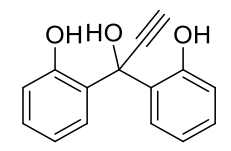

Following the general procedure F. 86\% yield. Syrup. ${ }^{1} \mathbf{H}$ NMR $\left(500 \mathrm{MHz}, \mathrm{CDCl}_{3}\right): \delta 7.61(\mathrm{~s}$, 
2H), $7.27(\mathrm{td}, J=8.01 .0 \mathrm{~Hz}, 2 \mathrm{H}), 7.10(\mathrm{dd}, J=8.01 .5 \mathrm{~Hz}, 2 \mathrm{H}), 6.93(\mathrm{dd}, J=8.01 .0 \mathrm{~Hz}, 2 \mathrm{H})$, $6.86(\mathrm{td}, J=7.51 .0 \mathrm{~Hz}, 2 \mathrm{H}), 5.42(\mathrm{~s}, 1 \mathrm{H}), 2.98(\mathrm{~s}, 1 \mathrm{H}) .{ }^{13} \mathbf{C} \mathbf{N M R}\left(75 \mathrm{MHz}, \mathrm{CDCl}_{3}\right): \delta$ 154.68, 130.63, 128.09, 125.85, 120.26, 117.59, 82.59, 77.55, 76.42. HRMS (ESI) m/z Calcd for $\left[\mathrm{C}_{15} \mathrm{H}_{12} \mathrm{NaO}_{3}, \mathrm{M}+\mathrm{Na}\right]^{+}:$263.0678; Found 263.0674.

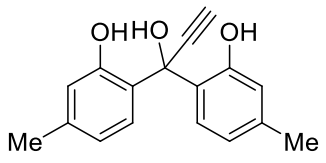

Following the general procedure F. $90 \%$ yield. Syrup. ${ }^{1} \mathbf{H}$ NMR (500 MHz, acetone-d $\left.\mathrm{d}_{6}\right): \delta$ $8.17(\mathrm{br}, 2 \mathrm{H}), 7.01(\mathrm{~d}, J=8.0 \mathrm{~Hz}, 2 \mathrm{H}), 6.72(\mathrm{~s}, 2 \mathrm{H}), 6.64(\mathrm{~d}, J=8.0 \mathrm{~Hz}, 2 \mathrm{H}), 3.45(\mathrm{~s}, 1 \mathrm{H})$, 2.27 (s, 6H). ${ }^{13} \mathbf{C}$ NMR (125 MHz, acetone-d $\left.\mathrm{d}_{6}\right): \delta 155.44,139.89,127.83,124.53,119.91$, 117.57, 84.05, 76.79, 75.70, 20.15. HRMS (ESI) m/z Calcd for $\left[\mathrm{C}_{17} \mathrm{H}_{16} \mathrm{NaO}_{3}, \mathrm{M}+\mathrm{Na}\right]^{+}$: 291.0991; Found 291.0995.

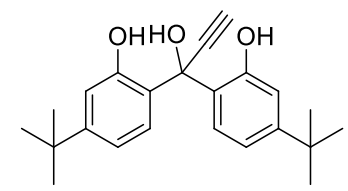

Following the general procedure F. 85\% yield. Syrup. ${ }^{1} \mathbf{H}$ NMR (500 MHz, acetone-d $\left.\mathrm{d}_{6}\right): \delta$ $8.23(\mathrm{br}, 2 \mathrm{H}), 7.09$ (d, $J=8.0 \mathrm{~Hz}, 2 \mathrm{H}), 6.94(\mathrm{~d}, J=1.5 \mathrm{~Hz}, 2 \mathrm{H}), 6.89(\mathrm{dd}, J=8.01 .5 \mathrm{~Hz}, 2 \mathrm{H})$, $3.43(\mathrm{~s}, 1 \mathrm{H}), 1.31(\mathrm{~s}, 18 \mathrm{H}) .{ }^{13} \mathrm{C}$ NMR $(125 \mathrm{MHz}$, acetone-d 6$): \delta 155.17,153.23,127.65$, 124.37, 116.21, 114.16, 84.10, 76.72, 75.62, 34.12, 30.66. HRMS (ESI) m/z Calcd for $\left[\mathrm{C}_{23} \mathrm{H}_{28} \mathrm{NaO}_{3}, \mathrm{M}+\mathrm{Na}\right]^{+}:$375.1930; Found 375.1936.

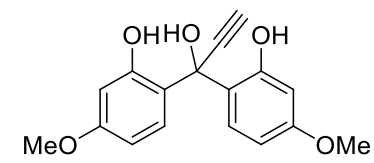

Following the general procedure F. $80 \%$ yield. Syrup. ${ }^{1} \mathbf{H}$ NMR (300 MHz, acetone-d $\left.\mathrm{d}_{6}\right): \delta$ $8.10(\mathrm{br}, 2 \mathrm{H}), 7.03(\mathrm{~d}, J=8.4 \mathrm{~Hz}, 2 \mathrm{H}), 6.46(\mathrm{~d}, J=2.7 \mathrm{~Hz}, 2 \mathrm{H}), 6.41(\mathrm{dd}, J=8.42 .9 \mathrm{~Hz}, 2 \mathrm{H})$, 3.77 (s, 6H), 3.45 (s, 1H). ${ }^{13}$ C NMR (75 MHz, acetone- $\left.\mathrm{d}_{6}\right): \delta 161.17,156.66,128.62,119.83$, 104.55, 102.43, 84.08, 76.54, 75.25, 54.55. HRMS (ESI) $\mathrm{m} / \mathrm{z}$ Calcd for $\left[\mathrm{C}_{17} \mathrm{H}_{16} \mathrm{NaO}_{5}, \mathrm{M}+\right.$ $\mathrm{Na}^{+}:$323.0890; Found 323.0894. 


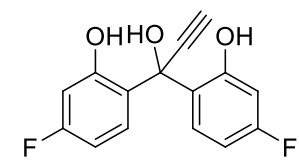

Following the general procedure F. $80 \%$ yield. Syrup. ${ }^{1} \mathbf{H}$ NMR $\left(500 \mathrm{MHz}, \mathrm{CDCl}_{3}\right): \delta 8.12(\mathrm{~s}$, 2H), $7.06(\mathrm{dd}, J=8.56 .5 \mathrm{~Hz}, 2 \mathrm{H}), 6.69(\mathrm{dd}, J=9.52 .5 \mathrm{~Hz}, 2 \mathrm{H}), 6.58(\mathrm{td}, J=8.52 .5 \mathrm{~Hz}, 2 \mathrm{H})$, $5.80(\mathrm{~s}, 1 \mathrm{H}), 3.01(\mathrm{~s}, 1 \mathrm{H}) .{ }^{13} \mathrm{C} \mathbf{N M R}\left(125 \mathrm{MHz}, \mathrm{CDCl}_{3}\right): \delta 164.08(\mathrm{~d}, J=246.25 \mathrm{~Hz}), 156.58$ $(\mathrm{d}, J=12.5 \mathrm{~Hz}), 129.27(\mathrm{~d}, J=10.0 \mathrm{~Hz}), 121.95(\mathrm{~d}, J=3.75 \mathrm{~Hz}), 106.94(\mathrm{~d}, J=21.25 \mathrm{~Hz})$, $105.17(\mathrm{~d}, J=25.00 \mathrm{~Hz}), 82.49,77.69,75.74$. HRMS (ESI) $\mathrm{m} / \mathrm{z}$ Calcd for $\left[\mathrm{C}_{15} \mathrm{H}_{10} \mathrm{~F}_{2} \mathrm{NaO}_{3}, \mathrm{M}\right.$ $+\mathrm{Na}]^{+}:$299.0490; Found 299.0495.

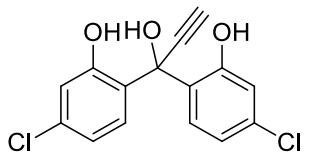

Following the general procedure F. $88 \%$ yield. Syrup. ${ }^{1} \mathbf{H}$ NMR (500 MHz, $\left.\mathrm{CDCl}_{3}\right): \delta 8.02$ (br, 2H), $7.02(\mathrm{~d}, J=8.5 \mathrm{~Hz}, 2 \mathrm{H}), 6.87(\mathrm{~d}, J=2.0 \mathrm{~Hz}, 2 \mathrm{H}), 6.86(\mathrm{dd}, J=8.52 .0 \mathrm{~Hz}, 2 \mathrm{H}), 5.81$ (br, 1H), $3.02(\mathrm{~s}, 1 \mathrm{H}) .{ }^{13} \mathrm{C}$ NMR $\left(125 \mathrm{MHz}, \mathrm{CDCl}_{3}\right): \delta 155.51,136.18,129.00,124.31$, 120.54, 118.03, 81.99, 78.19, 75.76. HRMS (ESI) m/z Calcd for $\left[\mathrm{C}_{15} \mathrm{H}_{10} \mathrm{Cl}_{2} \mathrm{NaO}_{3}, \mathrm{M}+\mathrm{Na}\right]^{+}$: 330.9899; Found 330.9896.

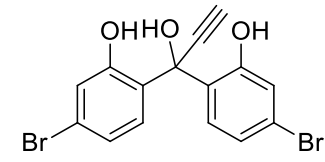

Following the general procedure F. $92 \%$ yield. Syrup. ${ }^{1} \mathbf{H}$ NMR $\left(500 \mathrm{MHz}, \mathrm{CDCl}_{3}\right): \delta 8.11(\mathrm{~s}$, 2H), $7.12(\mathrm{~d}, J=2.0 \mathrm{~Hz}, 2 \mathrm{H}), 7.01(\mathrm{dd}, J=8.02 .0 \mathrm{~Hz}, 2 \mathrm{H}), 6.96(\mathrm{~d}, J=8.0 \mathrm{~Hz}, 2 \mathrm{H}), 5.94(\mathrm{~s}$, 1H), $3.01(\mathrm{~s}, 1 \mathrm{H}) .{ }^{13} \mathrm{C}$ NMR $\left(125 \mathrm{MHz}, \mathrm{CDCl}_{3}\right): \delta$ 155.62, 129.24, 124.85, 124.05, 123.41, 120.91, 81.89, 78.10, 75.84. HRMS (ESI) $\mathrm{m} / \mathrm{z}$ Calcd for $\left[\mathrm{C}_{15} \mathrm{H}_{10} \mathrm{Br}_{2} \mathrm{NaO}_{3}, \mathrm{M}+\mathrm{Na}\right]^{+}$: 418.8889; Found 418.8892.

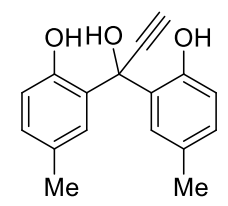

Following the general procedure F. 95\% yield. Syrup. ${ }^{1} \mathbf{H}$ NMR $\left(500 \mathrm{MHz}, \mathrm{CDCl}_{3}\right): \delta 7.67(\mathrm{~s}$, 2H), $7.08(\mathrm{dd}, J=8.02 .0 \mathrm{~Hz}, 2 \mathrm{H}), 6.92(\mathrm{~d}, J=2.0 \mathrm{~Hz}, 2 \mathrm{H}), 6.82(\mathrm{dd}, J=8.04 .0 \mathrm{~Hz}, 2 \mathrm{H})$, $5.75(\mathrm{~s}, 1 \mathrm{H}), 2.97(\mathrm{~s}, 1 \mathrm{H}), 2.24(\mathrm{~s}, 6 \mathrm{H}) .{ }^{13} \mathrm{C}$ NMR $\left(125 \mathrm{MHz}, \mathrm{CDCl}_{3}\right): \delta$ 152.45, 131.05, 
129.48, 128.45, 125.77, 117.45, 83.03, 77.40, 76.44, 20.65. HRMS (ESI) m/z Calcd for $\left[\mathrm{C}_{17} \mathrm{H}_{16} \mathrm{NaO}_{3}, \mathrm{M}+\mathrm{Na}\right]^{+}:$291.0991; Found 291.0994.

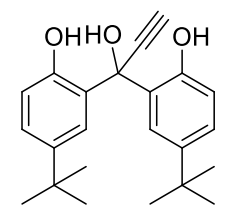

Following the general procedure F. 88\% yield. Syrup. ${ }^{1} \mathbf{H}$ NMR $\left(500 \mathrm{MHz}, \mathrm{CDCl}_{3}\right): \delta 7.78(\mathrm{~s}$, 2H), $7.29(\mathrm{dd}, J=8.52 .0 \mathrm{~Hz}, 2 \mathrm{H}), 7.10(\mathrm{~d}, J=2.0 \mathrm{~Hz}, 2 \mathrm{H}), 6.87(\mathrm{~d}, J=8.5 \mathrm{~Hz}, 2 \mathrm{H}), 5.82(\mathrm{~s}$, 1H), $2.99(\mathrm{~s}, 1 \mathrm{H}), 1.22(\mathrm{~s}, 18 \mathrm{H}),{ }^{13} \mathrm{C}$ NMR $\left(125 \mathrm{MHz}, \mathrm{CDCl}_{3}\right): \delta 152.42,142.85,127.17$, 125.28, 125.27, 116.90, 83.37, 77.09, 77.05, 34.10, 31.35. HRMS (ESI) m/z Calcd for $\left[\mathrm{C}_{23} \mathrm{H}_{28} \mathrm{NaO}_{3}, \mathrm{M}+\mathrm{Na}\right]^{+}:$375.1930; Found 375.1934.

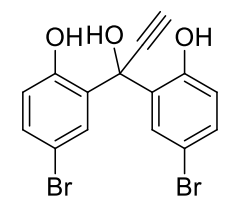

Following the general procedure F. 84\% yield. Syrup. ${ }^{1} \mathbf{H}$ NMR $\left(500 \mathrm{MHz}, \mathrm{CDCl}_{3}\right): \delta 7.81$ (s, 2H), $7.40(\mathrm{dd}, J=8.02 .0 \mathrm{~Hz}, 2 \mathrm{H}), 7.23(\mathrm{~d}, J=2.0 \mathrm{~Hz}, 2 \mathrm{H}), 6.84(\mathrm{~d}, J=8.04 .0 \mathrm{~Hz}, 2 \mathrm{H}), 5.75$ (s, 1H), 3.05 (s, 1H). ${ }^{13} \mathrm{C}$ NMR (125 MHz, $\left.\mathrm{CDCl}_{3}\right): \delta$ 153.95, 133.68, 130.60, 127.46, 119.61, 112.46, 81.57, 78.57, 75.44. HRMS (ESI) $\mathrm{m} / \mathrm{z}$ Calcd for $\left[\mathrm{C}_{15} \mathrm{H}_{10} \mathrm{Br}_{2} \mathrm{NaO}_{3}, \mathrm{M}+\mathrm{Na}\right]^{+}$: 418.8889; Found 418.8886.

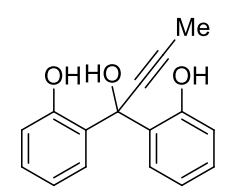

Following the general procedure F. 90\% yield. Syrup. ${ }^{1} \mathbf{H}$ NMR $\left(500 \mathrm{MHz}, \mathrm{CDCl}_{3}\right): \delta 7.97(\mathrm{~s}$, 2H), $7.26(\mathrm{td}, J=8.01 .0 \mathrm{~Hz}, 2 \mathrm{H}), 7.10(\mathrm{dd}, J=8.01 .0 \mathrm{~Hz}, 2 \mathrm{H}), 6.93(\mathrm{dd}, J=8.01 .0 \mathrm{~Hz}, 2 \mathrm{H})$, $6.86(\mathrm{td}, J=8.01 .0 \mathrm{~Hz}, 2 \mathrm{H}), 5.53(\mathrm{~s}, 1 \mathrm{H}), 1.96(\mathrm{~s}, 3 \mathrm{H}) .{ }^{13} \mathrm{C} \mathrm{NMR}\left(125 \mathrm{MHz}, \mathrm{CDCl}_{3}\right): \delta$ 154.89, 130.37, 128.15, 126.86, 120.07, 117.55, 86.44, 78.53, 76.87, 3.92. HRMS (ESI) m/z Calcd for $\left[\mathrm{C}_{16} \mathrm{H}_{14} \mathrm{NaO}_{3}, \mathrm{M}+\mathrm{Na}\right]^{+}:$277.0835; Found 277.0838.<smiles>COc1ccc(C(O)(O)c2ccccc2O)c(O)c1</smiles> 
Following the general procedure F. 80\% yield. Syrup. ${ }^{1} \mathbf{H}$ NMR $\left(300 \mathrm{MHz}, \mathrm{CDCl}_{3}\right): \delta 7.99$ (br, 1H), 7.92 (br, 1H), 7.29 (td, $J=7.81 .5 \mathrm{~Hz}, 1 \mathrm{H}), 7.13$ (dd, $J=7.81 .5 \mathrm{~Hz}, 1 \mathrm{H}), 7.01$ (d, $J$ $=8.7 \mathrm{~Hz}, 1 \mathrm{H}), 6.95(\mathrm{dd}, J=8.11 .2 \mathrm{~Hz}, 1 \mathrm{H}), 6.88(\mathrm{td}, J=7.81 .2 \mathrm{~Hz}, 1 \mathrm{H}), 6.53(\mathrm{~d}, J=2.7 \mathrm{~Hz}$, 1H), $6.42(\mathrm{dd}, J=8.72 .7 \mathrm{~Hz}, 1 \mathrm{H}), 5.82(\mathrm{br}, 1 \mathrm{H}), 3.79(\mathrm{~s}, 3 \mathrm{H}), 2.98(\mathrm{~s}, 1 \mathrm{H}) .{ }^{13} \mathbf{C}$ NMR $(75$ $\left.\mathrm{MHz}, \mathrm{CDCl}_{3}\right): \delta 161.40,156.14,154.83,130.49,128.91,128.03,126.14,120.08,118.39$, 117.49, 105.74, 103.01, 82.91, 77.12, 76.08, 55.26. HRMS (ESI) m/z Calcd for $\left[\mathrm{C}_{16} \mathrm{H}_{14} \mathrm{NaO}_{4}\right.$, $\mathrm{M}+\mathrm{Na}]^{+}:$293.0784; Found 293.0788.

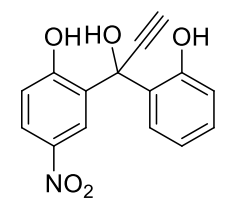

Following the general procedure F. $78 \%$ yield. Syrup. ${ }^{1} \mathbf{H}$ NMR (300 MHz, $\left.\mathrm{CDCl}_{3}\right): \delta 9.16$ (s, 1H), $8.19(\mathrm{dd}, J=9.02 .7 \mathrm{~Hz}, 1 \mathrm{H}), 8.03(\mathrm{~d}, J=2.7 \mathrm{~Hz}, 1 \mathrm{H}), 7.61(\mathrm{~s}, 1 \mathrm{H}), 7.36-7.30(\mathrm{~m}, 1 \mathrm{H})$, $7.18(\mathrm{dd}, J=8.11 .5 \mathrm{~Hz}, 1 \mathrm{H}), 7.04(\mathrm{~d}, J=9.0 \mathrm{~Hz}, 1 \mathrm{H}), 6.98-6.92(\mathrm{~m}, 2 \mathrm{H}), 6.19(\mathrm{~s}, 1 \mathrm{H}), 3.07$ (s, 1H). ${ }^{13} \mathbf{C}$ NMR $\left(75 \mathrm{MHz}, \mathrm{CDCl}_{3}\right): \delta$ 161.40, 153.93, 140.48, 131.09, 127.91, 126.69, 126.36, 125.23, 124.39, 120.86, 118.24, 117.49, 81.59, 78.29, 75.92. HRMS (ESI) m/z Calcd for $\left[\mathrm{C}_{15} \mathrm{H}_{11} \mathrm{NNaO}_{5}, \mathrm{M}+\mathrm{Na}\right]^{+}:$308.0529; Found 308.0526.

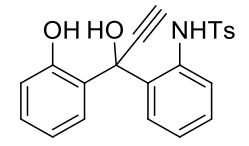

Following the general procedure F. $86 \%$ yield. Syrup. ${ }^{1} \mathbf{H}$ NMR (300 MHz, $\left.\mathrm{CDCl}_{3}\right): \delta 8.71$ (s, 1H), $7.71(\mathrm{~s}, 1 \mathrm{H}), 7.67(\mathrm{~d}, J=8.1 \mathrm{~Hz}, 1 \mathrm{H}), 7.60(\mathrm{~d}, J=8.1 \mathrm{~Hz}, 2 \mathrm{H}) 7.33-7.24(\mathrm{~m}, 3 \mathrm{H}), 7.18$ $(\mathrm{d}, J=8.1 \mathrm{~Hz}, 2 \mathrm{H}), 7.00(\mathrm{td}, J=7.51 .2 \mathrm{~Hz}, 1 \mathrm{H}), 6.95(\mathrm{dd}, J=8.11 .2 \mathrm{~Hz}, 1 \mathrm{H}), 6.83(\mathrm{dd}, J=$ $7.51 .8 \mathrm{~Hz}, 1 \mathrm{H}), 6.72(\mathrm{td}, J=7.51 .2 \mathrm{~Hz}, 1 \mathrm{H}), 5.44(\mathrm{~s}, 1 \mathrm{H}), 2.93(\mathrm{~s}, 1 \mathrm{H}), 2.40(\mathrm{~s}, 3 \mathrm{H}) .{ }^{13} \mathrm{C}$ NMR (125 MHz, $\left.\mathrm{CDCl}_{3}\right): \delta 154.85,143.75,136.57,136.39,130.56,130.08,129.56,128.87$, $128.60,127.85,127.39,125.76,123.31,120.36,119.62,117.78,82.66,78.33,76.33,21.53$.

HRMS (ESI) m/z Calcd for $\left[\mathrm{C}_{22} \mathrm{H}_{19} \mathrm{NNaO}_{4} \mathrm{~S}, \mathrm{M}+\mathrm{Na}\right]^{+}$: 416.0927; Found 416.0929.

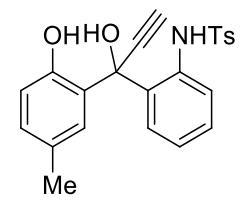


Following the general procedure F. $90 \%$ yield. Syrup. ${ }^{1} \mathbf{H}$ NMR $\left(500 \mathrm{MHz}, \mathrm{CDCl}_{3}\right): \delta 8.76$ (s, $1 \mathrm{H}), 7.64(\mathrm{~d}, J=8.0 \mathrm{~Hz}, 1 \mathrm{H}), 7.58-7.56(\mathrm{~m}, 3 \mathrm{H}), 7.30-7.26(\mathrm{~m}, 2 \mathrm{H}), 7.16(\mathrm{~d}, J=8.0 \mathrm{~Hz}, 2 \mathrm{H})$, $7.04(\mathrm{dd}, J=8.02 .0 \mathrm{~Hz}, 1 \mathrm{H}), 6.99(\mathrm{t}, J=8.0 \mathrm{~Hz}, 1 \mathrm{H}), 6.82(\mathrm{~d}, J=8.0 \mathrm{~Hz}, 1 \mathrm{H}), 6.63(\mathrm{~d}, J=$ $2.0 \mathrm{~Hz}, 1 \mathrm{H}), 5.55$ (s, 1H), $2.91(\mathrm{~s}, 1 \mathrm{H}), 2.37$ (s, 3H), 2.10 (s, 3H). ${ }^{13} \mathbf{C} \mathbf{N M R}\left(75 \mathrm{MHz}, \mathrm{CDCl}_{3}\right)$ : $\delta 152.42,143.71,136.58,136.32,131.07,130.01,129.54,128.85,128.26,127.34,125.52$, 123.29, 119.57, 117.62, 82.78, 78.34, 76.31, 21.52, 20.58. HRMS (ESI) m/z Calcd for $\left[\mathrm{C}_{23} \mathrm{H}_{21} \mathrm{NNaO}_{4} \mathrm{~S}, \mathrm{M}+\mathrm{Na}\right]^{+}:$430.1083; Found 430.1085 .

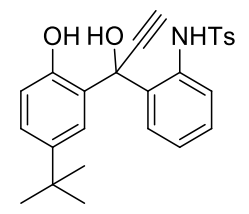

Following the general procedure F. 84\% yield. Syrup. ${ }^{1} \mathbf{H}$ NMR $\left(500 \mathrm{MHz}, \mathrm{CDCl}_{3}\right): \delta 8.83(\mathrm{~s}$, 1H), $7.70(\mathrm{dd}, J=8.5 \mathrm{~Hz}, 2 \mathrm{H}), 7.60(\mathrm{~d}, J=8.0 \mathrm{~Hz}, 1 \mathrm{H}), 7.36(\mathrm{~s}, 1 \mathrm{H}), 7.30-7.23(\mathrm{~m}, 3 \mathrm{H})$, $7.10(\mathrm{~d}, J=8.0 \mathrm{~Hz}, 2 \mathrm{H}) 7.02(\mathrm{dd}, J=8.01 .0 \mathrm{~Hz}, 1 \mathrm{H}), 6.93(\mathrm{t}, J=8.0 \mathrm{~Hz}, 1 \mathrm{H}), 6.84(\mathrm{~d}, J=$ 8.5Hz, 1H), $5.48(\mathrm{~s}, 1 \mathrm{H}), 2.86(\mathrm{~s}, 1 \mathrm{H}), 2.37$ (s, 3H), 1.23 (s, 9H). ${ }^{13} \mathrm{C}$ NMR (125 MHz, $\left.\mathrm{CDCl}_{3}\right): \delta 152.14,143.77,143.13,136.95,136.47,129.82,129.57,129.36,128.37,127.43$, $127.42,125.25,124.97,123.30,119.62,117.18,82.77,78.22,76.25,34.15,31.37,21.52$. HRMS (ESI) m/z Calcd for $\left[\mathrm{C}_{26} \mathrm{H}_{27} \mathrm{NNaO}_{4} \mathrm{~S}, \mathrm{M}+\mathrm{Na}\right]^{+}: 472.1553$; Found 472.1555.

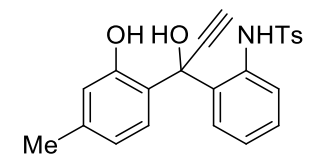

Following the general procedure F. 85\% yield. Syrup. ${ }^{1} \mathbf{H}$ NMR $\left(500 \mathrm{MHz}, \mathrm{CDCl}_{3}\right): \delta 8.69(\mathrm{~s}$, 1H), $7.67(\mathrm{dd}, J=9.01 .0 \mathrm{~Hz}, 1 \mathrm{H}), 7.59-7.57(\mathrm{~m}, 3 \mathrm{H}), 7.30(\mathrm{~d}, J=7.5 \mathrm{~Hz}, 2 \mathrm{H}), 7.16(\mathrm{~d}, J=$ $8.5 \mathrm{~Hz}, 2 \mathrm{H}), 6.99$ (t, $J=7.5 \mathrm{~Hz}, 1 \mathrm{H}), 6.74(\mathrm{~s}, 1 \mathrm{H}), 6.66(\mathrm{~d}, J=8.0 \mathrm{~Hz}, 1 \mathrm{H}), 6.48(\mathrm{~d}, J=7.0$ $\mathrm{Hz}, 1 \mathrm{H}), 5.33$ (s, 1H), 2.90 (s, 1H), 2.38 (s, 3H), 2.31 (s, 3H). $\left.{ }^{13} \mathbf{C ~ N M R ~ ( 1 2 5 ~ M H z , ~} \mathrm{CDCl}_{3}\right): \delta$ $154.58,143.67,140.87,136.67,136.40,129.99,129.46,128.89,128.81,127.62,127.38$, 123.29, 122.93, 121.17, 119.62, 118.30, 82.85, 78.15, 76.21, 21.51, 21.08. HRMS (ESI) m/z Calcd for $\left[\mathrm{C}_{23} \mathrm{H}_{21} \mathrm{NNaO}_{4} \mathrm{~S}, \mathrm{M}+\mathrm{Na}\right]^{+}:$430.1083; Found 430.1086 . 


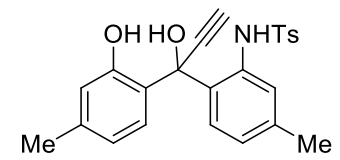

Following the general procedure F. 90\% yield. Syrup. ${ }^{1} \mathbf{H}$ NMR $\left(500 \mathrm{MHz}, \mathrm{CDCl}_{3}\right): \delta 8.67(\mathrm{~s}$, 1H), $7.73(\mathrm{~s}, 1 \mathrm{H}), 7.57$ (d, $J=8.5 \mathrm{~Hz}, 2 \mathrm{H}), 7.48(\mathrm{~s}, 1 \mathrm{H}), 7.50(\mathrm{~s}, 1 \mathrm{H}), 7.16-7.14(\mathrm{~m}, 3 \mathrm{H})$, $6.79(\mathrm{~d}, J=8.0 \mathrm{~Hz}, 1 \mathrm{H}) 6.72(\mathrm{~s}, 1 \mathrm{H}), 6.64(\mathrm{~d}, J=8.0 \mathrm{~Hz}, 1 \mathrm{H}), 6.45(\mathrm{~d}, J=8.0 \mathrm{~Hz}, 1 \mathrm{H}), 5.51$ (s, 1H), 2.88 (s, 1H), 2.38 (s, 3H), 2.31 (s, 3H), 2.30 (s, 3H). $\left.{ }^{13} \mathbf{C ~ N M R ~ ( 1 2 5 ~ M H z , ~} \mathrm{CDCl}_{3}\right): \delta$ $154.64,143.61,140.73,140.21,136.66,136.18,129.43,128.77,127.60,127.38,126.01$, $123.99,123.11,121.07,120.19,118.22,83.03,77.89,76.02,21.54,21.33,21.10$. HRMS (ESI) $\mathrm{m} / \mathrm{z}$ Calcd for $\left[\mathrm{C}_{24} \mathrm{H}_{23} \mathrm{NNaO}_{4} \mathrm{~S}, \mathrm{M}+\mathrm{Na}\right]^{+}$: 444.1240; Found 444.1244.

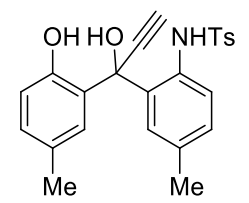

Following the general procedure F. 90\% yield. Syrup. ${ }^{1} \mathbf{H}$ NMR $\left(500 \mathrm{MHz}, \mathrm{CDCl}_{3}\right): \delta 8.53(\mathrm{~s}$, 1H), $7.57-7.52(\mathrm{~m}, 4 \mathrm{H}), 7.16(\mathrm{~d}, J=8.0 \mathrm{~Hz}, 2 \mathrm{H}), 7.10-7.09(\mathrm{~m}, 2 \mathrm{H}), 7.04(\mathrm{~d}, J=8.0 \mathrm{~Hz}$, 1H), 6.83 - $6.81(\mathrm{~m}, 1 \mathrm{H}), 6.60(\mathrm{~s}, 1 \mathrm{H}), 5.40(\mathrm{~s}, 1 \mathrm{H}), 2.92(\mathrm{~s}, 1 \mathrm{H}), 2.38(\mathrm{~s}, 3 \mathrm{H}), 2.23(\mathrm{~s}, 3 \mathrm{H})$, 2.11 (s, 3H). ${ }^{13} \mathbf{C}$ NMR $\left(75 \mathrm{MHz}, \mathrm{CDCl}_{3}\right): \delta$ 152.50, 143.55, 136.72, 133.64, 133.09, 131.08, $130.57,129.48,129.47,129.36,128.96,128.19,127.35,125.53,120.11,117.68,82.96,78.30$, 76.29, 21.51, 20.86, 20.59. HRMS (ESI) $\mathrm{m} / \mathrm{z}$ Calcd for $\left[\mathrm{C}_{24} \mathrm{H}_{23} \mathrm{NNaO}_{4} \mathrm{~S}, \mathrm{M}+\mathrm{Na}\right]^{+}: 444.1240$; Found 444.1242.

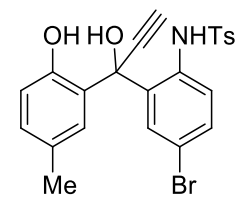

Following the general procedure F. $82 \%$ yield. Syrup. ${ }^{1} \mathbf{H}$ NMR $\left(300 \mathrm{MHz}, \mathrm{CDCl}_{3}\right): \delta 8.81(\mathrm{~s}$, 1H), $7.64(\mathrm{~s}, 1 \mathrm{H}), 7.58-7.53(\mathrm{~m}, 3 \mathrm{H}), 7.45-7.38(\mathrm{~m}, 2 \mathrm{H}), 7.17$ (d, J=8.1 Hz, 2H), $7.06(\mathrm{dd}$, $J=8.11 .8 \mathrm{~Hz}, 1 \mathrm{H}), 6.84(\mathrm{~d}, J=8.1 \mathrm{~Hz}, 1 \mathrm{H}), 6.58(\mathrm{~d}, J=1.5 \mathrm{~Hz}, 1 \mathrm{H}), 5.91(\mathrm{~s}, 1 \mathrm{H}), 2.92(\mathrm{~s}$, 1H), 2.39 (s, 3H), 2.11 (s, 3H). ${ }^{13} \mathbf{C}$ NMR (75 MHz, $\left.\mathrm{CDCl}_{3}\right): \delta 152.09,143.85,136.10,135.44$, $132.67,131.51,131.17,130.89,129.68,129.54,127.93,127.22,124.97,120.76,117.53$, 115.99, 82.20, 78.52, 75.64, 21.46, 20.52. HRMS (ESI) $\mathrm{m} / \mathrm{z}$ Calcd for $\left[\mathrm{C}_{23} \mathrm{H}_{20}{ }^{79} \mathrm{BrNNaO}_{4} \mathrm{~S}\right.$, $\mathrm{M}+\mathrm{Na}]^{+}:$508.0188; Found 508.0186; $\left[\mathrm{C}_{23} \mathrm{H}_{20}{ }^{81} \mathrm{BrNNaO}_{4} \mathrm{~S}, \mathrm{M}+\mathrm{Na}\right]^{+}:$510.0168; Found 
510.0166.

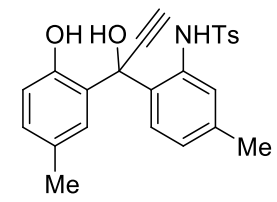

Following the general procedure F. 80\% yield. Syrup. ${ }^{1} \mathbf{H}$ NMR $\left(500 \mathrm{MHz}, \mathrm{CDCl}_{3}\right): \delta 8.65(\mathrm{~s}$, $1 \mathrm{H}), 7.58(\mathrm{~d}, J=8.5 \mathrm{~Hz}, 2 \mathrm{H}), 7.52(\mathrm{~s}, 1 \mathrm{H}), 7.48(\mathrm{~s}, 1 \mathrm{H}), 7.16(\mathrm{~d}, J=8.5 \mathrm{~Hz}, 2 \mathrm{H}), 7.11(\mathrm{~d}, J=$ $8.0 \mathrm{~Hz}, 1 \mathrm{H}), 7.04(\mathrm{dd}, J=8.02 .0 \mathrm{~Hz}, 1 \mathrm{H}) 6.82(\mathrm{~d}, J=8.5 \mathrm{~Hz}, 1 \mathrm{H}), 6.78(\mathrm{dd}, J=8.01 .5 \mathrm{~Hz}$, $1 \mathrm{H}), 6.65(\mathrm{~d}, J=2.0 \mathrm{~Hz}, 1 \mathrm{H}), 5.36(\mathrm{~s}, 1 \mathrm{H}), 2.89$ (s, 1H), $2.38(\mathrm{~s}, 3 \mathrm{H}), 2.30(\mathrm{~s}, 3 \mathrm{H}), 2.11$ (s, 3H). ${ }^{13} \mathrm{C}$ NMR $\left(125 \mathrm{MHz}, \mathrm{CDCl}_{3}\right): \delta 152.56,143.57,140.23,136.72,136.13,131.01,129.46$, 129.41, 128.67, 128.25, 127.35, 126.03, 125.60, 124.00, 120.31, 117.60, 82.98, 78.07, 76.15, 21.51, 21.32, 20.57. HRMS (ESI) $\mathrm{m} / \mathrm{z}$ Calcd for $\left[\mathrm{C}_{24} \mathrm{H}_{23} \mathrm{NNaO}_{4} \mathrm{~S}, \mathrm{M}+\mathrm{Na}\right]^{+}$: 444.1240; Found 444.1246. 


\section{Synthesis of benzofuran-derived bridged biaryls.}

\subsection{Optimization studies}

The initial screening studies were conducted with 2,2'-(1-hydroxyprop-2-yne-1,1-diyl) diphenol 1a and $(E)$-hex-2-enal 2c under the catalysis of A1 and DIPEA in DCM at $24{ }^{\circ} \mathrm{C}$. The desired benzofuran-fused eight-membered lactone $\mathbf{4} \mathbf{c}$ was isolated in $76 \%$ yield with a good enantioselectivity (90\% ee) after 36 h (entry 1, Table S1). Subsequently, other chiral NHC precursors were tested (entries 2-4, Table S1), and NHC precursor A3 improved both the yield and the enantioselectivity of this process (entry 3, Table S1). Other bases and other solvents were also explored, which led to inferior results (entries 5-9, Table S1).

Table S1. Screening conditions of the synthesis of benzofuran-derived bridged biaryls.<smiles>C#CC(O)(c1ccccc1O)c1ccccc1O</smiles>

1a

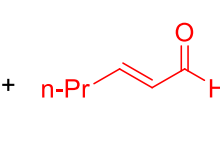

2c

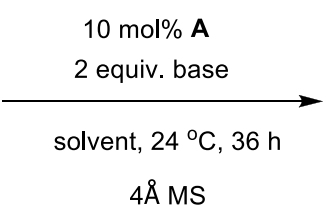

$4 \AA$ MS

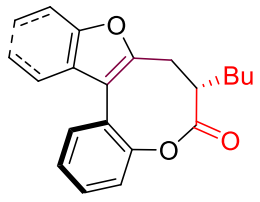

$(M, S)$ - 4c

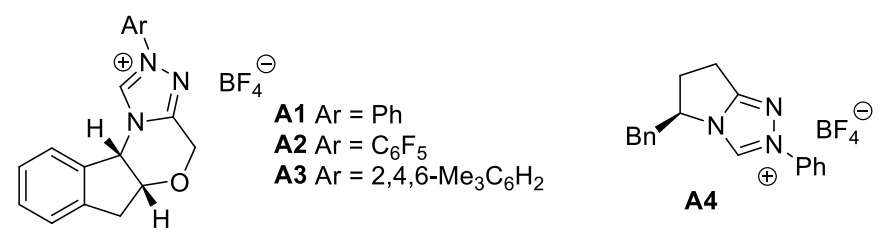

\begin{tabular}{cccccc}
\hline Entry & Cat. & Solvent & Base & 4c Yield $(\%)^{*}$ & 4c ee $(\%)^{\dagger}$ \\
\hline 1 & $\mathbf{A 1}$ & DCM & DIPEA & 76 & 90 \\
2 & $\mathbf{A 2}$ & DCM & DIPEA & 72 & 80 \\
$\mathbf{3}$ & $\mathbf{A 3}$ & DCM & DIPEA & $\mathbf{8 5}$ & $\mathbf{9 9}$ \\
4 & $\mathbf{A 4}$ & DCM & DIPEA & trace & n.d. \\
5 & $\mathbf{A 3}$ & DCM & NaOAc & trace & n.d. \\
6 & $\mathbf{A 3}$ & DCM & $\mathrm{Cs}_{2} \mathrm{CO}_{3}$ & trace & n.d. \\
7 & $\mathbf{A 3}$ & toluene & DIPEA & 70 & 97 \\
8 & $\mathbf{A 3}$ & $\mathrm{CHCl}$ & DIPEA & 70 & 98 \\
9 & $\mathbf{A 3}$ & THF & DIPEA & 65 & 98 \\
\hline
\end{tabular}

Reactions were performed with $1 \mathbf{a}(0.15 \mathrm{mmol}, 1.0$ equiv. $), 2 \mathbf{c}(0.60 \mathrm{mmol}, 4.0$ equiv. $)$, cat. A (10 mol\%), base (0.30 mmol, 2.0 equiv.) and $4 \AA$ molecular sevie $(100 \mathrm{mg})$ in solvent $(2.0 \mathrm{~mL})$ at $24{ }^{\circ} \mathrm{C}$ for 36 h. *Isolated yield. $†$ Determined by HPLC analysis on a chiral stationary phase. n.d. = not determined. 


\subsection{General procedure for the synthesis of benzofuran-derived bridged biaryls}

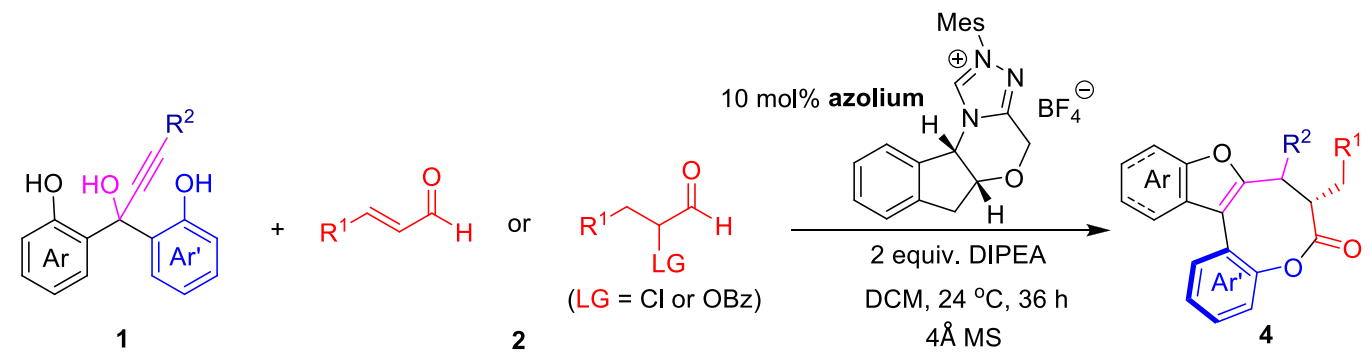

To a $4 \mathrm{~mL}$ vial was added the triol $1(0.15 \mathrm{mmol})$ and azolium $(0.015 \mathrm{mmol})$. The mixture was taken into the glovebox, where aldehyde $2(0.60 \mathrm{mmol}), 4 \AA$ molecular sieve $(100 \mathrm{mg})$, anhydrous DCM $(2.0 \mathrm{~mL})$ and $N, N$-diisopropylethylamine $(0.30 \mathrm{mmol})$ were added. The reaction mixture was taken outside the glovebox and the reaction mixture was allowed to stir at $24{ }^{\circ} \mathrm{C}$ for $36 \mathrm{~h}$. The crude reaction mixture was directly purified by silica gel column chromatography with hexanes/ethyl acetate $(20: 1 \mathrm{v} / \mathrm{v})$ as eluent to afford the product 4 in pure form.

Note: in certain cases, the preparation of the corresponding racemic products for HPLC analysis required the use of the racemic catalyst.

\subsection{Structure of aldehydes 2}<smiles>CC(C)C(C=O)C(C)C</smiles>

2a<smiles>CCCCCCC(Cl)C=O</smiles>

2<smiles>CC/C=C/C=O</smiles>

2b<smiles>CCC/C=C/C=O</smiles>

2c<smiles>CCCC/C=C/C=O</smiles>

2d<smiles>O=CC(Cl)Cc1ccccc1</smiles><smiles>C[13CH3]</smiles>

$2 g$

The aldehydes shown above were used to explore the scope of this system. For each product in the following section, the identity of the aldehyde used is included before the characterization data. 


\section{Characterization of benzofuran-derived bridged biaryls.}

(S)-7-isopropyl-7,8-dihydro-6H-benzo[2,3] oxocino[5,4-b]benzofuran-6-one. (Scheme 3, 4a)<smiles>CC(C)C1Cc2oc3ccccc3c2-c2ccccc2OC1=O</smiles>

Used 2-Benzoylisovaleraldehyde 2a. Syrup. ${ }^{1} \mathbf{H}$ NMR (300 MHz, $\left.\mathrm{CDCl}_{3}\right): \delta 7.62(\mathrm{dd}, J=7.2$ $2.4 \mathrm{~Hz}, 1 \mathrm{H}), 7.51-7.41(\mathrm{~m}, 4 \mathrm{H}), 7.36-7.23(\mathrm{~m}, 3 \mathrm{H}), 3.39(\mathrm{dd}, J=16.84 .8 \mathrm{~Hz}, 1 \mathrm{H}), 3.15$ $((\mathrm{dd}, J=16.813 .2 \mathrm{~Hz}, 1 \mathrm{H}), 2.99-2.92(\mathrm{~m}, 1 \mathrm{H}), 2.21-2.14(\mathrm{~m}, 1 \mathrm{H}), 1.02(\mathrm{~d}, J=5.7 \mathrm{~Hz}, 3 \mathrm{H})$, $1.00(\mathrm{~d}, J=5.7 \mathrm{~Hz}, 3 \mathrm{H}) .{ }^{13} \mathrm{C}$ NMR $\left(125 \mathrm{MHz}, \mathrm{CDCl}_{3}\right): \delta 173.74,154.36,151.60,149.43$, $131.42,129.42,128.75,126.23,124.67,124.51,122.93,121.46,119.22,111.97,110.88$, 45.24, 31.15, 31.06, 21.22, 19.36. HRMS (ESI) m/z Calcd for $\left[\mathrm{C}_{20} \mathrm{H}_{18} \mathrm{NaO}_{3}, \mathrm{M}+\mathrm{Na}\right]^{+}$: 329.1148; Found 329.1144.

Optical Rotation: $[\alpha]^{25}{ }_{\mathrm{D}}-20.0\left(c=0.3, \mathrm{CHCl}_{3}\right) .99 \%$ ee (HPLC condition: Chiralpak ID column, $n$-Hexane $/ i-\mathrm{PrOH}=99: 1$, flow rate $=1.0 \mathrm{~mL} / \mathrm{min}$, wave length $=254 \mathrm{~nm}, t_{\mathrm{R}}=12.93$ $\min$ for minor isomer, $t_{\mathrm{R}}=27.45 \mathrm{~min}$ for major isomer).

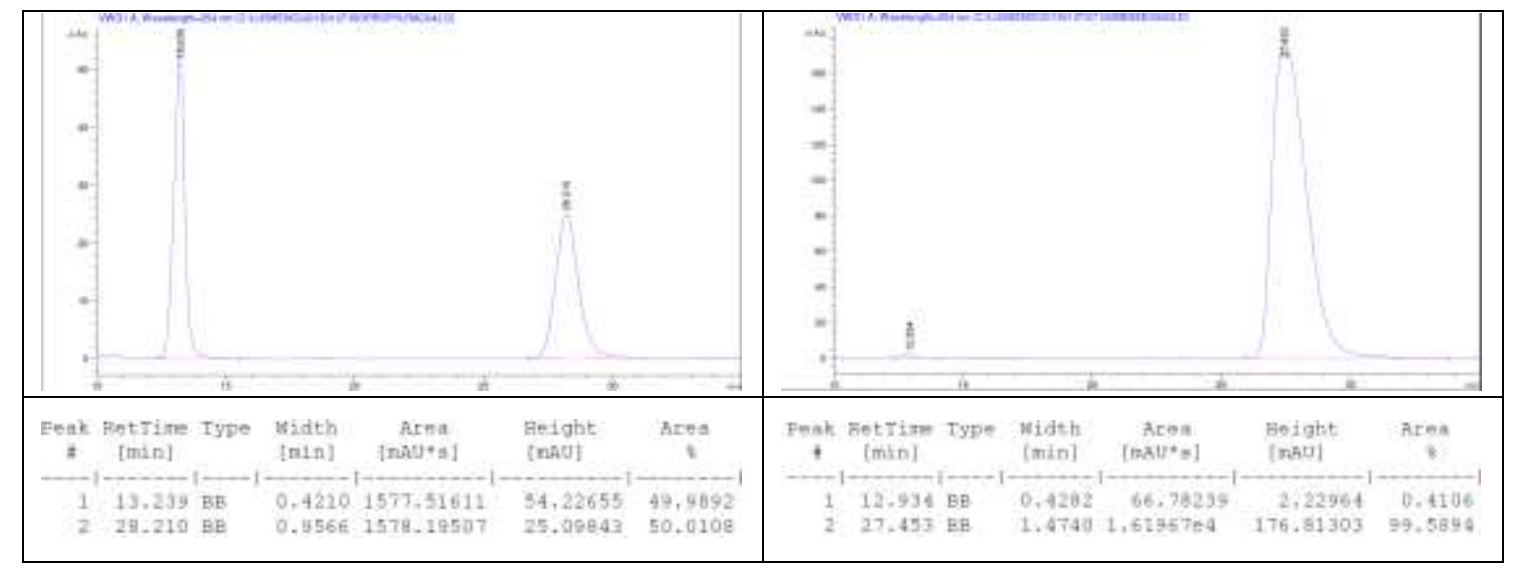

(S)-7-propyl-7,8-dihydro-6H-benzo[2,3] oxocino[5,4-b]benzofuran-6-one. (Scheme 4a, 4b)<smiles>CCCC(=O)OCc1ccccc1-c1coc2ccccc12</smiles>

Used Enal 2b. White solid. MP: $180-181{ }^{\circ} \mathrm{C} .{ }^{1} \mathbf{H}$ NMR $\left(500 \mathrm{MHz}, \mathrm{CDCl}_{3}\right): \delta 7.61(\mathrm{dd}, J=$ $7.52 .0 \mathrm{~Hz}, 1 \mathrm{H}), 7.47-7.40(\mathrm{~m}, 4 \mathrm{H}), 7.32(\mathrm{td}, J=7.51 .0 \mathrm{~Hz}, 1 \mathrm{H}), 7.29-7.28(\mathrm{~m}, 1 \mathrm{H}), 7.25$ $(\mathrm{t}, J=7.5 \mathrm{~Hz}, 1 \mathrm{H}), 3.30-3.16 \quad(\mathrm{~m}, 3 \mathrm{H}), 1.99-1.96(\mathrm{~m}, 1 \mathrm{H}), 1.53-1.50(\mathrm{~m}, 1 \mathrm{H}), 1.43-1.42$ $(\mathrm{m}, 1 \mathrm{H}), 1.31-1.27(\mathrm{~m}, 1 \mathrm{H}), 0.88(\mathrm{t}, J=7.0 \mathrm{~Hz}, 3 \mathrm{H}) .{ }^{13} \mathbf{C} \mathbf{N M R}\left(125 \mathrm{MHz}, \mathrm{CDCl}_{3}\right): \delta 173.89$, $154.41,151.26,149.39,131.48,129.43,128.78,126.45,124.86,124.63,123.03,121.74$, 119.31, 112.15, 111.00, 37.54, 34.92, 33.41, 20.60, 13.66. HRMS (ESI) $\mathrm{m} / \mathrm{z}$ Calcd for $\left[\mathrm{C}_{20} \mathrm{H}_{18} \mathrm{NaO}_{3}, \mathrm{M}+\mathrm{Na}\right]^{+}$: 329.1148; Found 329.1146. 
Optical Rotation: $[\alpha]^{25} \mathrm{D}-59.0\left(c=0.2, \mathrm{CHCl}_{3}\right) .99 \%$ ee (HPLC condition: Chiralpak ID column, $n$-Hexane $/ i-\mathrm{PrOH}=98: 2$, flow rate $=1.0 \mathrm{~mL} / \mathrm{min}$, wave length $=254 \mathrm{~nm}, t_{\mathrm{R}}=14.33$ $\min$ for minor isomer, $t_{\mathrm{R}}=27.21 \mathrm{~min}$ for major isomer).

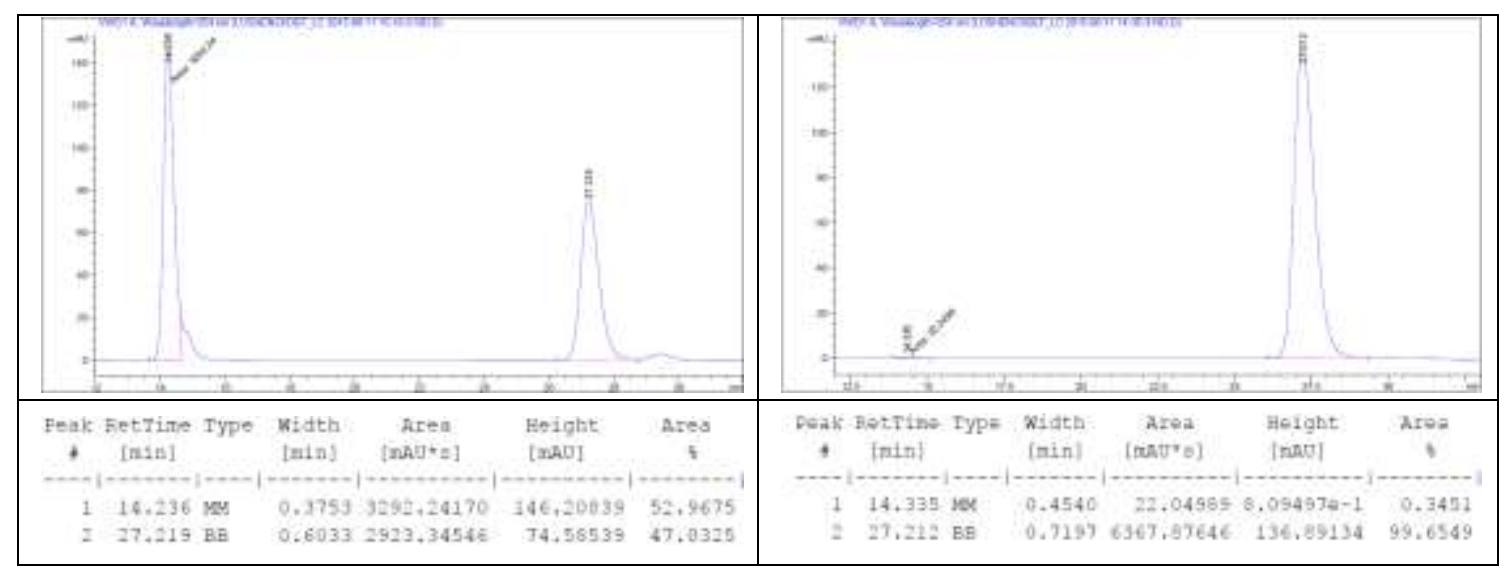

(S)-7-butyl-7,8-dihydro-6H-benzo[2,3]oxocino[5,4-b]benzofuran-6-one. (Scheme 4a, 4c)<smiles>O=C1Cc2oc3ccccc3c2-c2ccccc2CC1[18O]</smiles>

Used Enal 2c. White solid. MP: $108-109{ }^{\circ} \mathrm{C} .{ }^{1} \mathbf{H}$ NMR $\left(500 \mathrm{MHz}, \mathrm{CDCl}_{3}\right): \delta 7.61(\mathrm{dd}, J=$ $7.52 .0 \mathrm{~Hz}, 1 \mathrm{H}), 7.48-7.40(\mathrm{~m}, 4 \mathrm{H}), 7.32(\mathrm{td}, J=7.51 .0 \mathrm{~Hz}, 1 \mathrm{H}), 7.28-7.26(\mathrm{~m}, 1 \mathrm{H}), 7.25$ $(\mathrm{t}, J=7.5 \mathrm{~Hz}, 1 \mathrm{H}), 3.28-3.16 \quad(\mathrm{~m}, 3 \mathrm{H}), 1.97-1.95(\mathrm{~m}, 1 \mathrm{H}), 1.56-1.54(\mathrm{~m}, 1 \mathrm{H}), 1.36-$ $1.23(\mathrm{~m}, 4 \mathrm{H}), 0.90(\mathrm{t}, J=7.0 \mathrm{~Hz}, 3 \mathrm{H}) .{ }^{13} \mathbf{C} \mathbf{~ N M R}\left(125 \mathrm{MHz}, \mathrm{CDCl}_{3}\right): \delta 173.92,154.41$, $151.27,149.40,131.47,129.44,128.78,126.44,124.84,124.62,123.03,121.73,119.30$, 112.14, 111.00, 37.85, 33.40, 32.65, 29.56, 22.37, 13.83. HRMS (ESI) m/z Calcd for $\left[\mathrm{C}_{21} \mathrm{H}_{20} \mathrm{NaO}_{3}, \mathrm{M}+\mathrm{Na}\right]^{+}:$343.1304; Found 343.1300.

Optical Rotation: $[\alpha]^{25}{ }_{\mathrm{D}}-46.0\left(c=0.3, \mathrm{CHCl}_{3}\right) .99 \%$ ee (HPLC condition: Chiralpak ID column, $n$-Hexane $/ i-\mathrm{PrOH}=98: 2$, flow rate $=1.0 \mathrm{~mL} / \mathrm{min}$, wave length $=254 \mathrm{~nm}, t_{\mathrm{R}}=12.11$ $\min$ for minor isomer, $t_{\mathrm{R}}=24.75 \mathrm{~min}$ for major isomer).

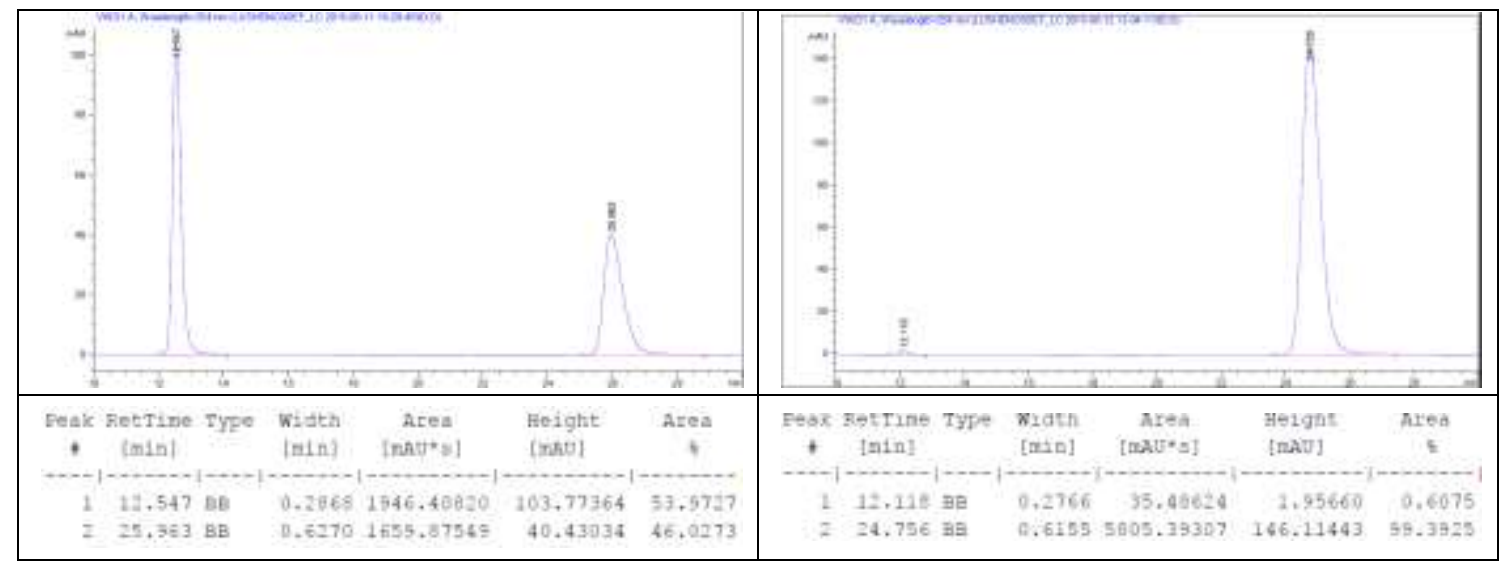


(S)-7-pentyl-7,8-dihydro-6H-benzo[2,3]oxocino[5,4-b]benzofuran-6-one. (Scheme 4a, 4d)<smiles>O=C1Cc2oc3ccccc3c2-c2ccccc2O1</smiles>

Used Enal 2d. Syrup. ${ }^{1} \mathbf{H}$ NMR $\left(500 \mathrm{MHz}, \mathrm{CDCl}_{3}\right): \delta 7.61(\mathrm{dd}, J=7.51 .5 \mathrm{~Hz}, 1 \mathrm{H}), 7.48-$ $7.40(\mathrm{~m}, 3 \mathrm{H}), 7.42(\mathrm{td}, J=7.51 .0 \mathrm{~Hz}, 1 \mathrm{H}), 7.32(\mathrm{t}, J=8.0 \mathrm{~Hz}, 1 \mathrm{H}), 7.29-7.28(\mathrm{~m}, 1 \mathrm{H})$, $7.25(\mathrm{t}, J=7.5 \mathrm{~Hz}, 1 \mathrm{H}), 3.28-3.13(\mathrm{~m}, 3 \mathrm{H}), 1.97-1.95(\mathrm{~m}, 1 \mathrm{H}), 1.54-1.44(\mathrm{~m}, 1 \mathrm{H}), 1.38$ $1.22(\mathrm{~m}, 6 \mathrm{H}), 0.88(\mathrm{t}, J=7.0 \mathrm{~Hz}, 3 \mathrm{H}) .{ }^{13} \mathbf{C}$ NMR $\left(125 \mathrm{MHz}, \mathrm{CDCl}_{3}\right): \delta 173.92,154.40$, $151.27,149.39,131.47,129.42,128.79,126.44,124.86,124.62,123.03,121.75,119.31$, 112.14, 111.00, 37.83, 33.41, 32.87, 31.40, 27.04, 22.38, 13.96. HRMS (ESI) m/z Calcd for $\left[\mathrm{C}_{22} \mathrm{H}_{22} \mathrm{NaO}_{3}, \mathrm{M}+\mathrm{Na}\right]^{+}:$357.1461; Found 357.1464.

Optical Rotation: $[\alpha]^{25} \mathrm{D}-35.0\left(c=0.3, \mathrm{CHCl}_{3}\right) .99 \%$ ee (HPLC condition: Chiralpak ID column, $n$-Hexane $/ i-\mathrm{PrOH}=98: 2$, flow rate $=1.0 \mathrm{~mL} / \mathrm{min}$, wave length $=254 \mathrm{~nm}, t_{\mathrm{R}}=10.06$ $\min$ for minor isomer, $t_{\mathrm{R}}=19.11 \mathrm{~min}$ for major isomer).

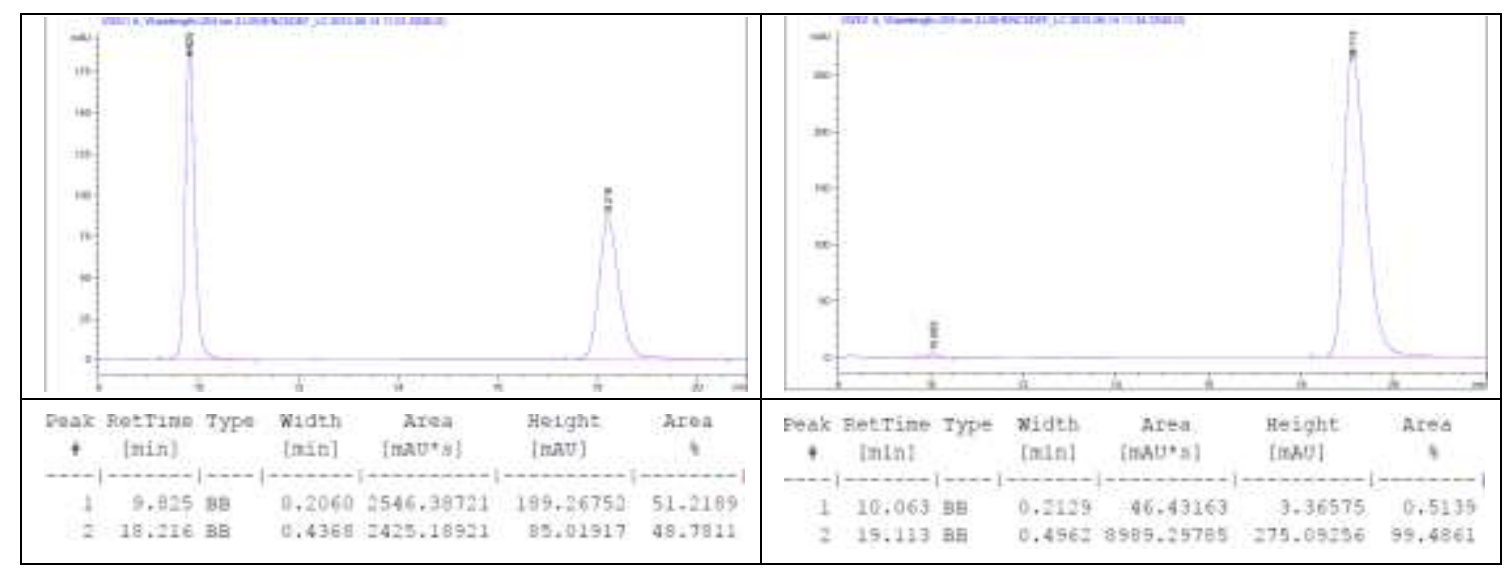

(S)-7-hexyl-7,8-dihydro-6H-benzo[2,3] oxocino[5,4-b]benzofuran-6-one. (Scheme 4a, 4e)

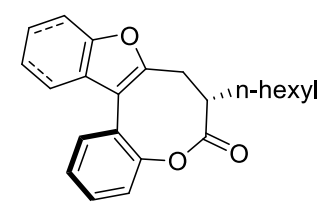

Used 2-Chloroaldehyde 2e. Syrup. ${ }^{1} \mathbf{H}$ NMR $\left(500 \mathrm{MHz}, \mathrm{CDCl}_{3}\right): \delta 7.61(\mathrm{dd}, J=7.52 .0 \mathrm{~Hz}$, 1H), $7.48-7.40(\mathrm{~m}, 4 \mathrm{H}), 7.32(\mathrm{td}, J=7.51 .0 \mathrm{~Hz}, 1 \mathrm{H}), 7.29-7.26(\mathrm{~m}, 1 \mathrm{H}), 7.25(\mathrm{t}, J=7.5$ $\mathrm{Hz}, 1 \mathrm{H}), 3.30-3.13(\mathrm{~m}, 3 \mathrm{H}), 1.98-1.96(\mathrm{~m}, 1 \mathrm{H}), 1.57-1.51(\mathrm{~m}, 1 \mathrm{H}), 1.37-1.25(\mathrm{~m}, 8 \mathrm{H})$, $0.89(\mathrm{t}, J=7.0 \mathrm{~Hz}, 3 \mathrm{H}) . \quad{ }^{13} \mathbf{C} \mathbf{N M R}\left(125 \mathrm{MHz}, \mathrm{CDCl}_{3}\right): \delta 173.88,154.41,151.28,149.41$, $131.47,129.41,128.79,126.42,124.87,124.61,123.03,121.76,119.30,112.14,111.00$, 37.80, 33.42, 32.91, 31.53, 28.85, 27.32, 22.50, 13.99. HRMS (ESI) m/z Calcd for $\left[\mathrm{C}_{23} \mathrm{H}_{24} \mathrm{NaO}_{3}, \mathrm{M}+\mathrm{Na}\right]^{+}:$371.1617; Found 371.1614.

Optical Rotation: $[\alpha]^{25}{ }_{\mathrm{D}}-60.0\left(c=0.2, \mathrm{CHCl}_{3}\right) .99 \%$ ee (HPLC condition: Chiralpak ID column, $n$-Hexane $/ i-\mathrm{PrOH}=98: 2$, flow rate $=1.0 \mathrm{~mL} / \mathrm{min}$, wave length $=254 \mathrm{~nm}, t_{\mathrm{R}}=10.49$ $\min$ for minor isomer, $t_{\mathrm{R}}=20.87 \mathrm{~min}$ for major isomer). 


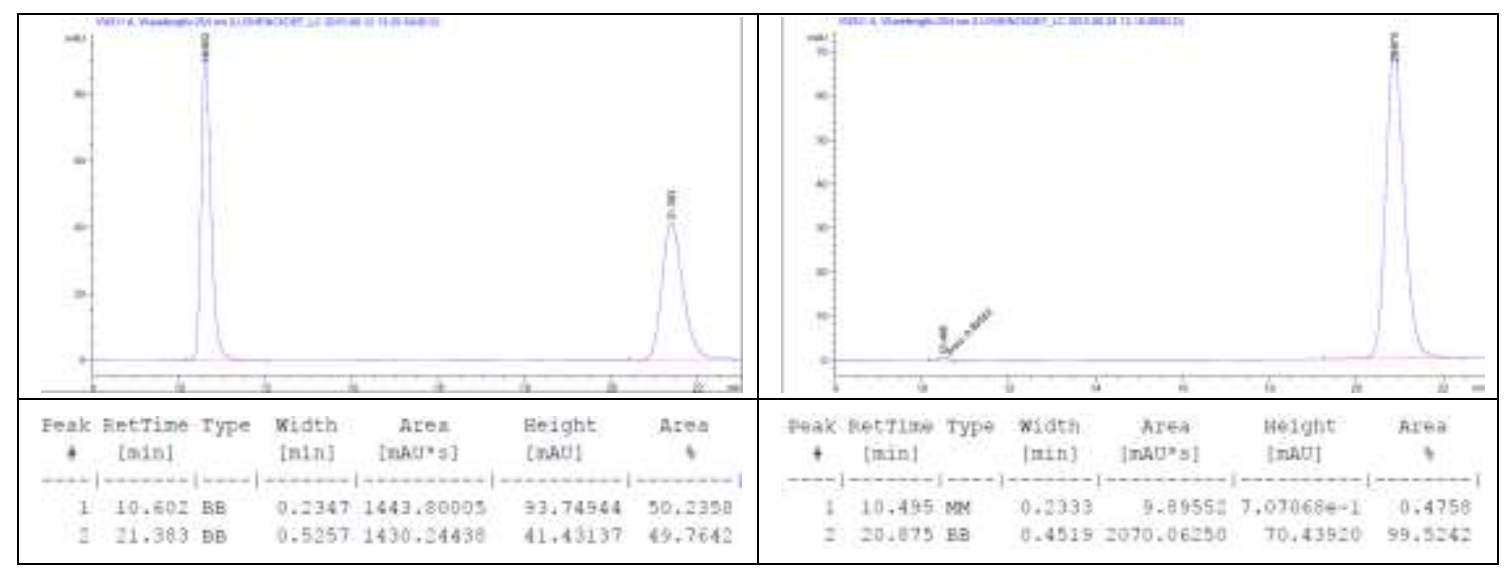

(S)-7-benzyl-7,8-dihydro-6H-benzo[2,3] oxocino[5,4-b]benzofuran-6-one. (Scheme 4a, 4f)<smiles>O=C1CCc2oc3ccccc3c2-c2ccccc2O1</smiles>

Used 2-Chloroaldehyde 2f. Syrup. ${ }^{1} \mathbf{H}$ NMR (500 MHz, $\left.\mathrm{CDCl}_{3}\right): \delta 7.59$ (dd, $J=6.03 .0 \mathrm{~Hz}$, $1 \mathrm{H}), 7.48-7.40(\mathrm{~m}, 4 \mathrm{H}), 7.34-7.28(\mathrm{~m}, 3 \mathrm{H}), 7.26-7.23(\mathrm{~m}, 2 \mathrm{H}), 7.12(\mathrm{~d}, J=7.0 \mathrm{~Hz}, 2 \mathrm{H})$, $7.05(\mathrm{dd}, J=6.03 .0 \mathrm{~Hz}, 1 \mathrm{H}), 3.64-3.59(\mathrm{~m}, 1 \mathrm{H}), 3.30-3.27(\mathrm{~m}, 2 \mathrm{H}), 3.22(\mathrm{dd}, J=14.09 .0$ $\mathrm{Hz}, 1 \mathrm{H}), 2.91(\mathrm{dd}, J=14.06 .0 \mathrm{~Hz}, 1 \mathrm{H}) .{ }^{13} \mathrm{C}$ NMR $\left(125 \mathrm{MHz}, \mathrm{CDCl}_{3}\right): \delta 173.10,154.43$, $150.90,149.19,137.94,131.43,129.43,128.69,128.63,128.62,126.82,126.50,124.70$, 124.68, 123.07, 121.75, 119.33, 112.23, 111.00, 39.51, 38.55, 33.02. HRMS (ESI) m/z Calcd for $\left[\mathrm{C}_{24} \mathrm{H}_{18} \mathrm{NaO}_{3}, \mathrm{M}+\mathrm{Na}\right]^{+}$: 377.1148; Found 377.1144.

Optical Rotation: $[\alpha]^{25} \mathrm{D}-20.0\left(c=0.1, \mathrm{CHCl}_{3}\right) .99 \%$ ee (HPLC condition: Chiralpak ID column, $n$-Hexane $/ i-\mathrm{PrOH}=95: 5$, flow rate $=1.0 \mathrm{~mL} / \mathrm{min}$, wave length $=254 \mathrm{~nm}, t_{\mathrm{R}}=14.39$ $\mathrm{min}$ for minor isomer, $t_{\mathrm{R}}=36.30 \mathrm{~min}$ for major isomer).

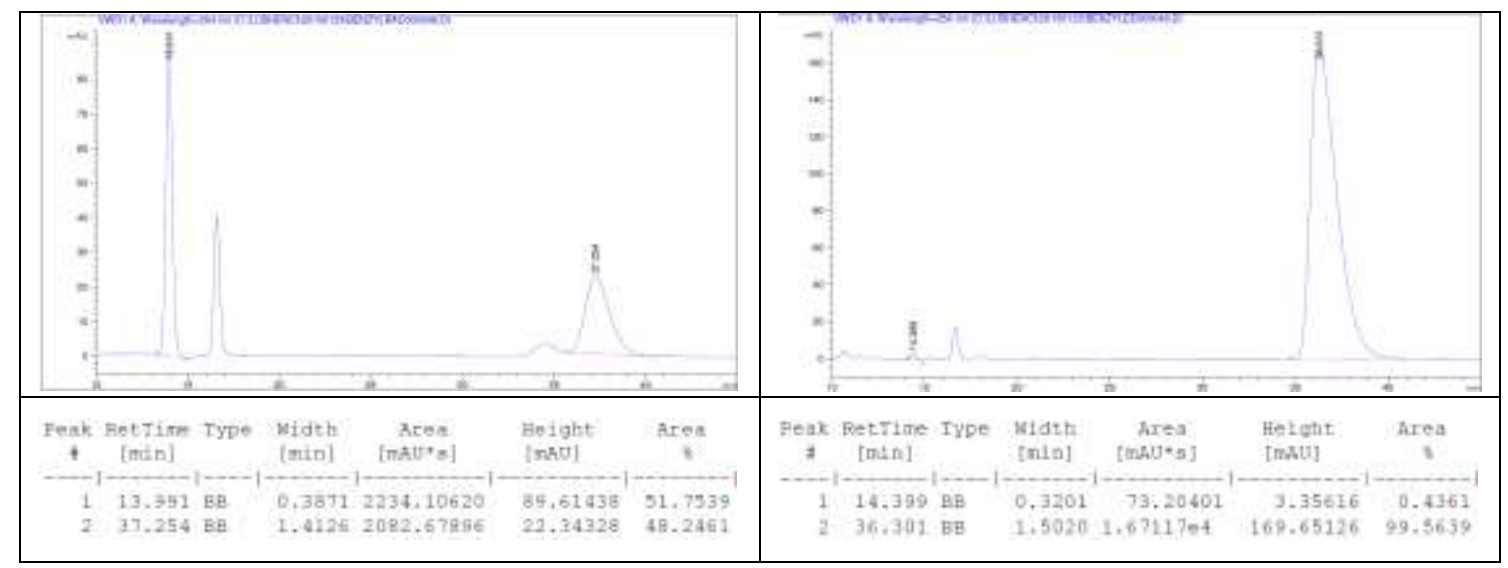

(S)-7-(3-((tert-butyldimethylsilyl)oxy)propyl)-7,8-dihydro-6H-benzo[2,3] oxocino[5,4-b]benz ofuran-6-one. (Scheme 4a, 4g) 


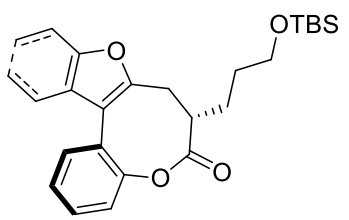

Used Enal 2g. Syrup. ${ }^{1} \mathbf{H}$ NMR $\left(500 \mathrm{MHz}, \mathrm{CDCl}_{3}\right): \delta 7.60(\mathrm{~d}, J=7.5 \mathrm{~Hz}, 1 \mathrm{H}), 7.47-7.40(\mathrm{~m}$, 4H), $7.32(\mathrm{t}, J=7.5 \mathrm{~Hz}, 1 \mathrm{H}), 7.28-7.27(\mathrm{~m}, 1 \mathrm{H}), 7.24(\mathrm{t}, J=7.5 \mathrm{~Hz}, 1 \mathrm{H}), 3.57-3.55(\mathrm{~m}$, 2H), 3.33 - $3.13(\mathrm{~m}, 3 \mathrm{H}), 1.96-1.91(\mathrm{~m}, 1 \mathrm{H}), 1.72-1.70(\mathrm{~m}, 1 \mathrm{H}), 1.69-1.63(\mathrm{~m}, 1 \mathrm{H}), 1.47$ $1.41(\mathrm{~m}, 1 \mathrm{H}), 0.86(\mathrm{~s}, 9 \mathrm{H}), 0.01(\mathrm{~s}, 3 \mathrm{H}), 0.006(\mathrm{~s}, 3 \mathrm{H}),{ }^{13} \mathrm{C}$ NMR $\left(125 \mathrm{MHz}, \mathrm{CDCl}_{3}\right): \delta$ $173.77,154.41,151.19,149.34,131.50,129.45,128.77,126.50,124.83,124.63,123.04$, 121.76, 119.31, 112.12, 111.01, 62.19, 37.47, 33.41, 30.28, 29.25, 25.92, 18.31, - 5.39. HRMS (ESI) $\mathrm{m} / \mathrm{z}$ Calcd for $\left[\mathrm{C}_{26} \mathrm{H}_{32} \mathrm{NaO}_{4} \mathrm{Si}, \mathrm{M}+\mathrm{Na}\right]^{+}:$459.1962; Found 459.1966.

Optical Rotation: $[\alpha]^{25} \mathrm{D}-68.0\left(c=0.2, \mathrm{CHCl}_{3}\right) .99 \%$ ee (HPLC condition: Chiralpak ID column, $n$-Hexane $/ i-\mathrm{PrOH}=98: 2$, flow rate $=1.0 \mathrm{~mL} / \mathrm{min}$, wave length $=254 \mathrm{~nm}, t_{\mathrm{R}}=7.77$ $\min$ for minor isomer, $t_{\mathrm{R}}=14.16 \mathrm{~min}$ for major isomer).

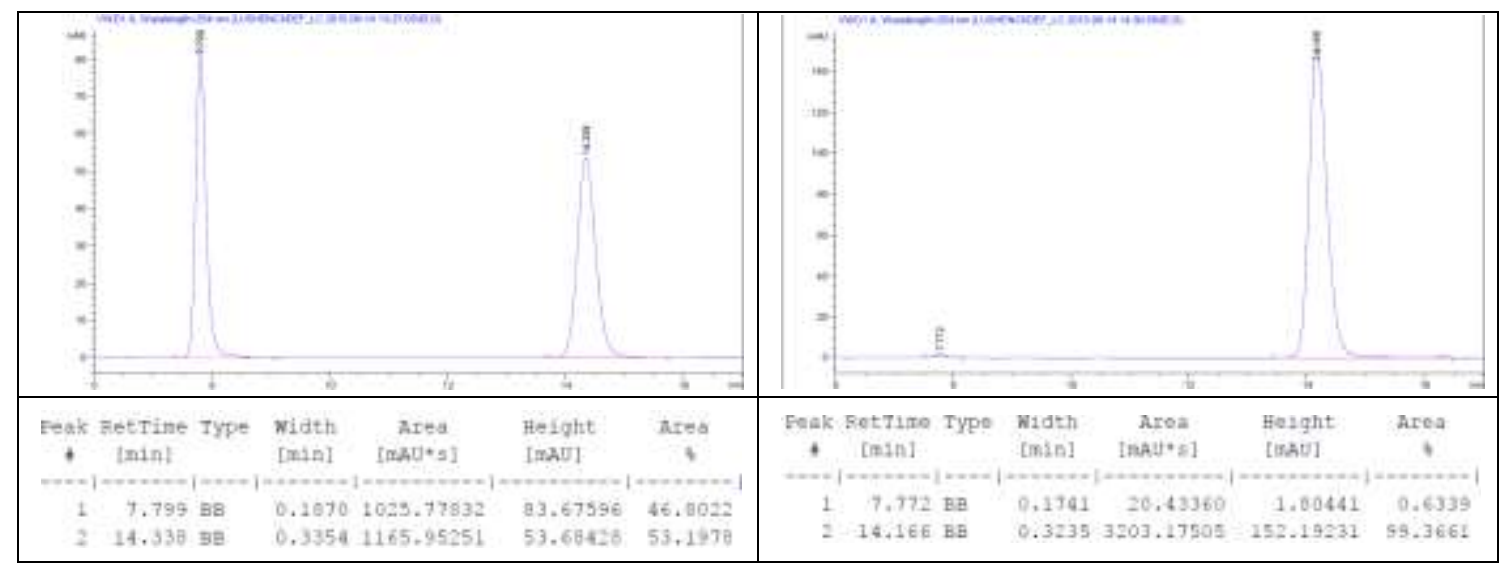

(S)-7-butyl-3,11-dimethyl-7,8-dihydro-6H-benzo[2,3] oxocino[5,4-b]benzofuran-6-one.

(Scheme 4a, 4h)<smiles>Cc1ccc2c(c1)OC(=O)C([18O])Cc1oc3cc([N+](=O)[O-])ccc3c1-2</smiles>

Used Enal 2c. Syrup. ${ }^{1} \mathbf{H}$ NMR $\left(500 \mathrm{MHz}, \mathrm{CDCl}_{3}\right): \delta 7.44(\mathrm{~d}, J=7.5 \mathrm{~Hz}, 1 \mathrm{H}), 7.27(\mathrm{~d}, J=8.0$ $\mathrm{Hz}, 1 \mathrm{H}), 7.23$ (s, 1H), $7.19(\mathrm{~d}, J=8.0 \mathrm{~Hz}, 1 \mathrm{H}), 7.06(\mathrm{~s}, 1 \mathrm{H}), 7.04(\mathrm{~d}, J=8.0 \mathrm{~Hz}, 1 \mathrm{H}), 3.26-$ $3.06(\mathrm{~m}, 3 \mathrm{H}), 2.46(\mathrm{~s}, 3 \mathrm{H}), 2.44(\mathrm{~s}, 3 \mathrm{H}), 1.94-1.88(\mathrm{~m}, 1 \mathrm{H}), 1.54-1.49(\mathrm{~m}, 1 \mathrm{H}), 1.35-1.19$ $(\mathrm{m}, 4 \mathrm{H}), 0.88(\mathrm{t}, J=7.0 \mathrm{~Hz}, 3 \mathrm{H}) .{ }^{13} \mathbf{C}$ NMR $\left(125 \mathrm{MHz}, \mathrm{CDCl}_{3}\right): \delta 174.11,154.78,150.36$, $149.15,139.76,134.78,131.09,127.17,126.42,124.26,122.06,121.84,118.81,111.92$, 111.17, 37.90, 33.36, 32.71, 29.55, 22.42, 21.59, 21.08, 13.81. HRMS (ESI) m/z Calcd for $\left[\mathrm{C}_{23} \mathrm{H}_{24} \mathrm{NaO}_{3}, \mathrm{M}+\mathrm{Na}\right]^{+}:$371.1617; Found 371.1613. 
Optical Rotation: $[\alpha]^{25} \mathrm{D}-37.0\left(c=0.1, \mathrm{CHCl}_{3}\right) .99 \%$ ee (HPLC condition: Chiralpak ID column, $n$-Hexane $/ i-\mathrm{PrOH}=98: 2$, flow rate $=1.0 \mathrm{~mL} / \mathrm{min}$, wave length $=254 \mathrm{~nm}, t_{\mathrm{R}}=13.74$ $\min$ for minor isomer, $t_{\mathrm{R}}=44.63 \mathrm{~min}$ for major isomer).

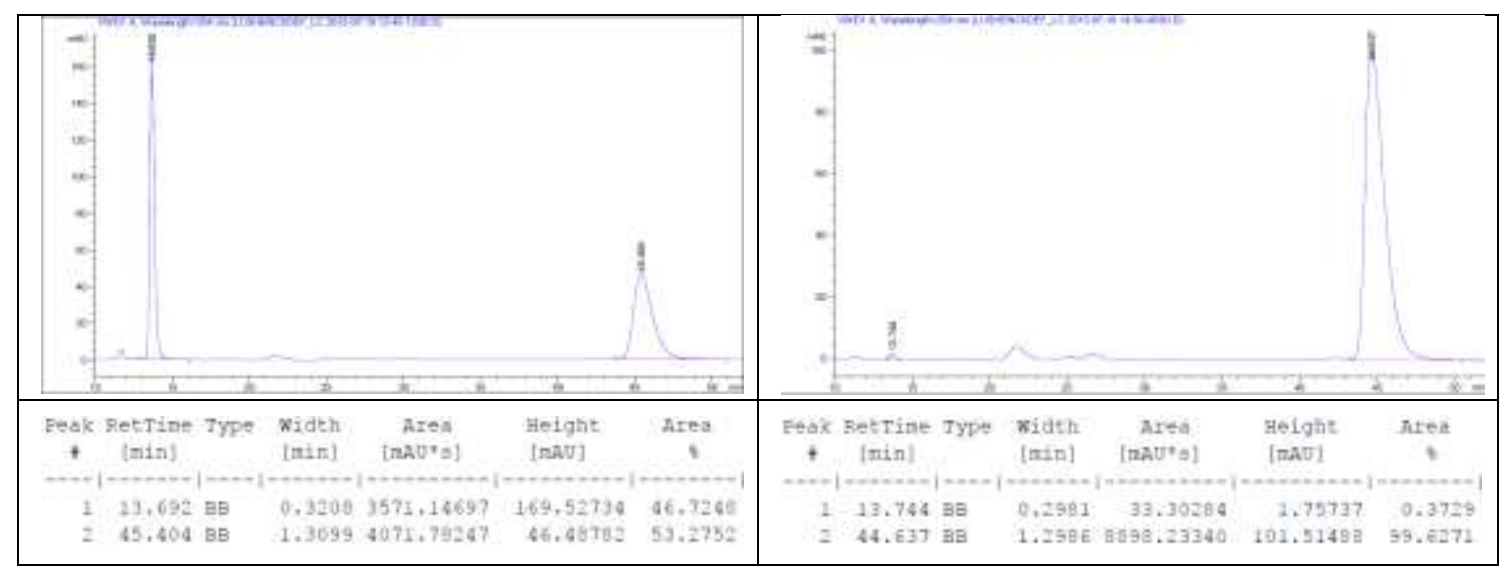

(S)-3,11-di-tert-butyl-7-butyl-7,8-dihydro-6H-benzo[2,3] oxocino[5,4-b] benzofuran-6-one.

(Scheme 4a, 4i)

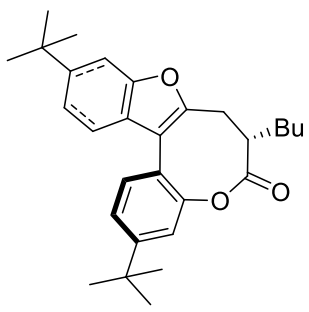

Used Enal 2c. White solid. MP: $144-145^{\circ} \mathrm{C} .{ }^{1} \mathbf{H}$ NMR $\left(500 \mathrm{MHz}, \mathrm{CDCl}_{3}\right): \delta 7.53(\mathrm{~d}, J=$ $8.0 \mathrm{~Hz}, 1 \mathrm{H}), 7.48(\mathrm{~d}, J=1.0 \mathrm{~Hz}, 1 \mathrm{H}), 7.42(\mathrm{dd}, J=8.02 .0 \mathrm{~Hz}, 1 \mathrm{H}), 7.39(\mathrm{~d}, J=8.5 \mathrm{~Hz}, 1 \mathrm{H})$, $7.31(\mathrm{dd}, J=8.01 .5 \mathrm{~Hz}, 1 \mathrm{H}), 7.28(\mathrm{~d}, J=2.0 \mathrm{~Hz}, 1 \mathrm{H}), 3.23-3.11(\mathrm{~m}, 3 \mathrm{H}), 2.01-1.94(\mathrm{~m}$, 1H), $1.57-1.50(\mathrm{~m}, 1 \mathrm{H}), 1.41(\mathrm{~s}, 9 \mathrm{H}), 1.40(\mathrm{~s}, 9 \mathrm{H}), 1.30-1.22(\mathrm{~m}, 4 \mathrm{H}), 0.89(\mathrm{t}, J=7.0 \mathrm{~Hz}$, 3H). ${ }^{13} \mathbf{C}$ NMR (125 MHz, $\left.\mathrm{CDCl}_{3}\right): \delta 174.08,154.76,153.27,150.81,149.12,148.62,130.84$, $126.30,123.30,121.87,120.64,118.72$, 118.67, 111.84, 107.68, 37.54, 34.95, 34.79, 33.49, $32.52,31.66,31.18,29.37,22.15,13.80$. HRMS (ESI) $\mathrm{m} / \mathrm{z}$ Calcd for $\left[\mathrm{C}_{29} \mathrm{H}_{36} \mathrm{NaO}_{3}, \mathrm{M}+\mathrm{Na}\right]^{+}$: 455.2556; Found 455.2558.

Optical Rotation: $[\alpha]^{25}-10.0\left(c=0.2, \mathrm{CHCl}_{3}\right) .99 \%$ ee (HPLC condition: Chiralpak ID column, $n$-Hexane $/ i-\mathrm{PrOH}=98: 2$, flow rate $=1.0 \mathrm{~mL} / \mathrm{min}$, wave length $=254 \mathrm{~nm}, t_{\mathrm{R}}=7.53$ $\min$ for minor isomer, $t_{\mathrm{R}}=18.19 \mathrm{~min}$ for major isomer).

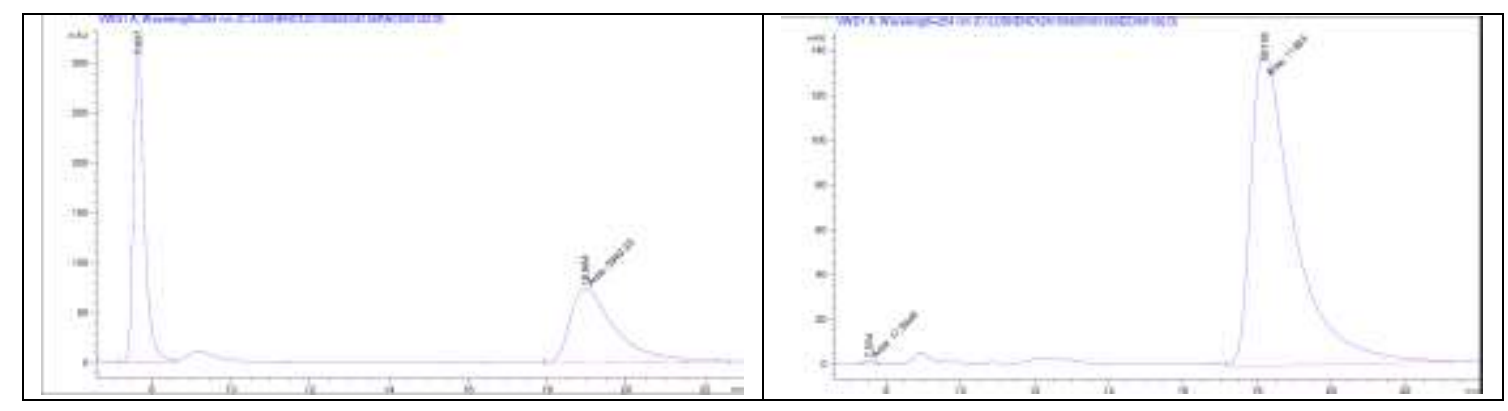




\begin{tabular}{|c|c|c|c|c|c|c|c|c|c|c|c|c|c|}
\hline $\begin{array}{c}\text { Fwak } \\
t\end{array}$ & $\begin{array}{l}\text { Retrino } \\
\text { [min] }\end{array}$ & TYDo & $\begin{array}{l}\text { Midtt } \\
\mid n\lfloor n\}\end{array}$ & $\begin{array}{c}\text { hrea } \\
{[n 200 \cdot 3]}\end{array}$ & $\begin{array}{l}\text { Height } \\
\text { [nauj] }\end{array}$ & Areas & Fenk & $\begin{array}{l}\text { Aatinge } \\
\text { [nin] }\end{array}$ & Trso & $\begin{array}{l}\text { Width. } \\
\text { [nin] }\end{array}$ & $\begin{array}{c}\text { Area } \\
\text { [nhu* }\end{array}$ & $\begin{array}{l}\text { Hetght } \\
\text { [RAty] }\end{array}$ & Aros \\
\hline 1 & 7.657 & $\mathrm{BV}$ & 0.2395 & 3997.07207 & 316.08170 & 20.1993 & 1 & 7,554 & NM & 0.2486 & 17,59414 & 1.17951 & 0.1545 \\
\hline 2 & 111.256 & wen & 1.3123 & 5940.33398 & 25.64546 & 49.8007 & 2 & 15,192 & MM & 1.3353 & $1.13630 \mathrm{e} 4$ & $142,+82449$ & 99,8654 \\
\hline
\end{tabular}

(S)-7-butyl-3,11-dimethoxy-7,8-dihydro-6H-benzo[2,3] oxocino[5,4- $b$ ]benzofuran-6-one.

(Scheme 4a, 4j)

$\mathrm{MeO}$<smiles>COc1ccc2c(c1)OC(=O)CCc1oc3ccccc3c1C2</smiles>

Used Enal 2c. White solid. MP: $140-141{ }^{\circ} \mathrm{C} .{ }^{1} \mathbf{H}$ NMR (500 MHz, $\left.\mathrm{CDCl}_{3}\right): \delta 7.47(\mathrm{~d}, J=$ $9.0 \mathrm{~Hz}, 1 \mathrm{H}), 7.27(\mathrm{~s}, 1 \mathrm{H}), 6.98(\mathrm{~s}, 1 \mathrm{H}), 6.96(\mathrm{dd}, J=8.52 .5 \mathrm{~Hz}, 1 \mathrm{H}), 6.87(\mathrm{dd}, J=9.02 .5 \mathrm{~Hz}$, $1 \mathrm{H}), 6.82(\mathrm{~d}, J=2.5 \mathrm{~Hz}, 1 \mathrm{H}), 3.90(\mathrm{~s}, 3 \mathrm{H}), 3.87(\mathrm{~s}, 3 \mathrm{H}), 3.28-3.08(\mathrm{~m}, 3 \mathrm{H}), 1.96-1.91(\mathrm{~m}$, 1H), $1.57-1.51(\mathrm{~m}, 1 \mathrm{H}), 1.38-1.22(\mathrm{~m}, 4 \mathrm{H}), 0.90(\mathrm{t}, J=7.0 \mathrm{~Hz}, 3 \mathrm{H}) .{ }^{13} \mathbf{C} \mathbf{N M R}(125 \mathrm{MHz}$, $\left.\mathrm{CDCl}_{3}\right): \delta 173.92,160.36,158.19,155.28,150.11,149.68,131.85,122.34,119.41,116.91$, 112.47, 111.69, 111.68, 107.12, 95.79, 55.78, 55.67, 37.99, 33.30, 32.69, 29.57, 22.40, 13.81 . HRMS (ESI) m/z Calcd for $\left[\mathrm{C}_{23} \mathrm{H}_{24} \mathrm{NaO}_{5}, \mathrm{M}+\mathrm{Na}\right]^{+}: 403.1516$; Found 403.1519.

Optical Rotation: $[\alpha]^{25} \mathrm{D}-55.0\left(c=0.1, \mathrm{CHCl}_{3}\right) .99 \%$ ee (HPLC condition: Chiralpak ID column, $n$-Hexane $/ i-\mathrm{PrOH}=80: 20$, flow rate $=1.0 \mathrm{~mL} / \mathrm{min}$, wave length $=254 \mathrm{~nm}, t_{\mathrm{R}}=$ $12.78 \mathrm{~min}$ for minor isomer, $t_{\mathrm{R}}=31.69 \mathrm{~min}$ for major isomer).

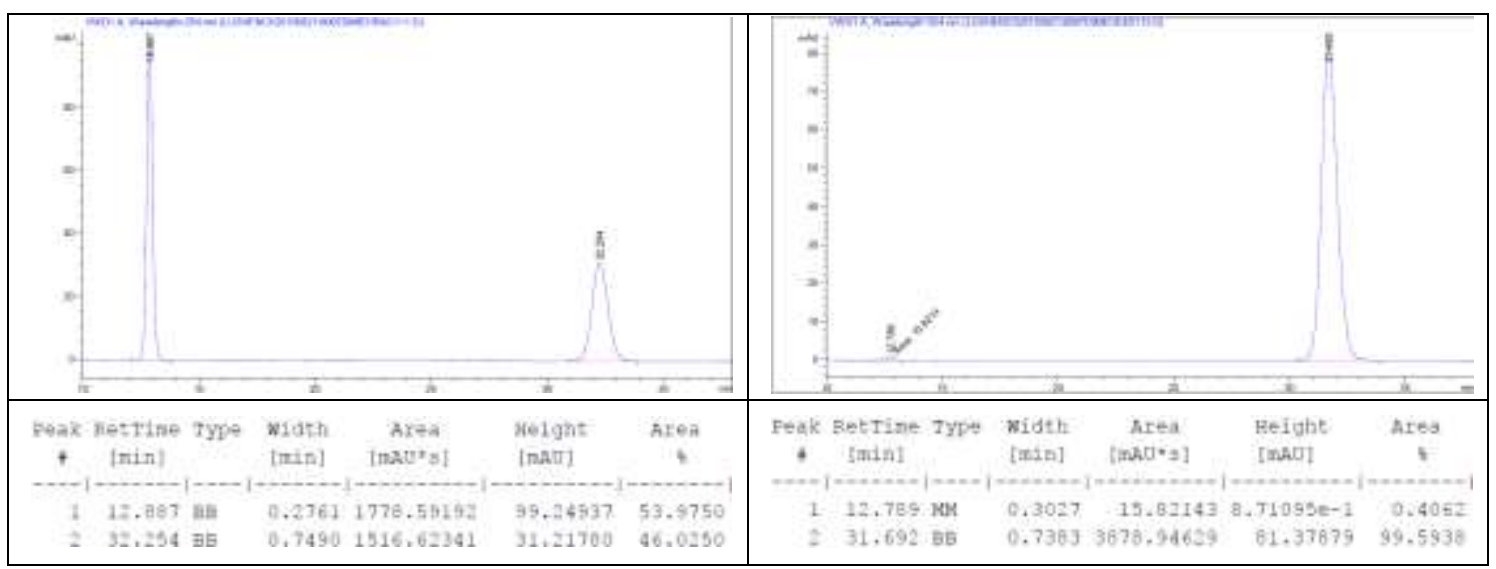

(S)-7-butyl-3,11-difluoro-7,8-dihydro-6H-benzo[2,3] oxocino[5,4-b]benzofuran-6-one.

(Scheme 4a, 4k)<smiles>CC(C)CCc1oc2cc(F)ccc2c1-c1ccc(F)cc1</smiles>

Used Enal 2c. Syrup. ${ }^{1} \mathbf{H}$ NMR $\left(500 \mathrm{MHz}, \mathrm{CDCl}_{3}\right): \delta 7.54(\mathrm{dd}, J=8.56 .5 \mathrm{~Hz}, 1 \mathrm{H}), 7.31(\mathrm{dd}$, $J=8.55 .0 \mathrm{~Hz}, 1 \mathrm{H}), 7.20-7.14(\mathrm{~m}, 2 \mathrm{H}), 7.06-7.00(\mathrm{~m}, 2 \mathrm{H}), 3.26-3.13(\mathrm{~m}, 3 \mathrm{H}), 1.96-1.92$ $(\mathrm{m}, 1 \mathrm{H}), 1.59-1.53(\mathrm{~m}, 1 \mathrm{H}), 1.37-1.22(\mathrm{~m}, 4 \mathrm{H}), 0.91(\mathrm{t}, J=7.0 \mathrm{~Hz}, 3 \mathrm{H}) .{ }^{13} \mathbf{C}$ NMR $(125$ 
$\left.\mathrm{MHz}, \mathrm{CDCl}_{3}\right): \delta 173.11,162.84(\mathrm{~d}, J=205.0 \mathrm{~Hz}), 160.87(\mathrm{~d}, J=197.5 \mathrm{~Hz}), 154.26(\mathrm{~d}, J=$ $13.75 \mathrm{~Hz}), 151.81$ (d, $J=5.0 \mathrm{~Hz}), 150.14$ (d, $J=10.0 \mathrm{~Hz}), 132.29$ (d, $J=10.0 \mathrm{~Hz}), 124.92$ (d, $J=1.25 \mathrm{~Hz}), 120.52(\mathrm{~d}, J=3.75 \mathrm{~Hz}), 119.45(\mathrm{~d}, J=10.0 \mathrm{~Hz}), 113.84(\mathrm{~d}, J=20.0 \mathrm{~Hz})$, 111.51, 111.30 (d, $J=3.75 \mathrm{~Hz}), 109.69$ (d, $J=23.75 \mathrm{~Hz}), 98.98$ (d, $J=26.25 \mathrm{~Hz}), 38.08$, 33.18, 32.66, 29.56, 22.39, 13.80. HRMS (ESI) $\mathrm{m} / \mathrm{z}$ Calcd for $\left[\mathrm{C}_{21} \mathrm{H}_{18} \mathrm{~F}_{2} \mathrm{NaO}_{3}, \mathrm{M}+\mathrm{Na}\right]^{+}$: 379.1116; Found 379.1119.

Optical Rotation: $[\alpha]^{25} \mathrm{D}-18.0\left(c=0.2, \mathrm{CHCl}_{3}\right) .97 \%$ ee (HPLC condition: Chiralpak ID column, $n$-Hexane $/ i-\mathrm{PrOH}=98: 2$, flow rate $=1.0 \mathrm{~mL} / \mathrm{min}$, wave length $=254 \mathrm{~nm}, t_{\mathrm{R}}=8.09$ $\mathrm{min}$ for minor isomer, $t_{\mathrm{R}}=11.69 \mathrm{~min}$ for major isomer).

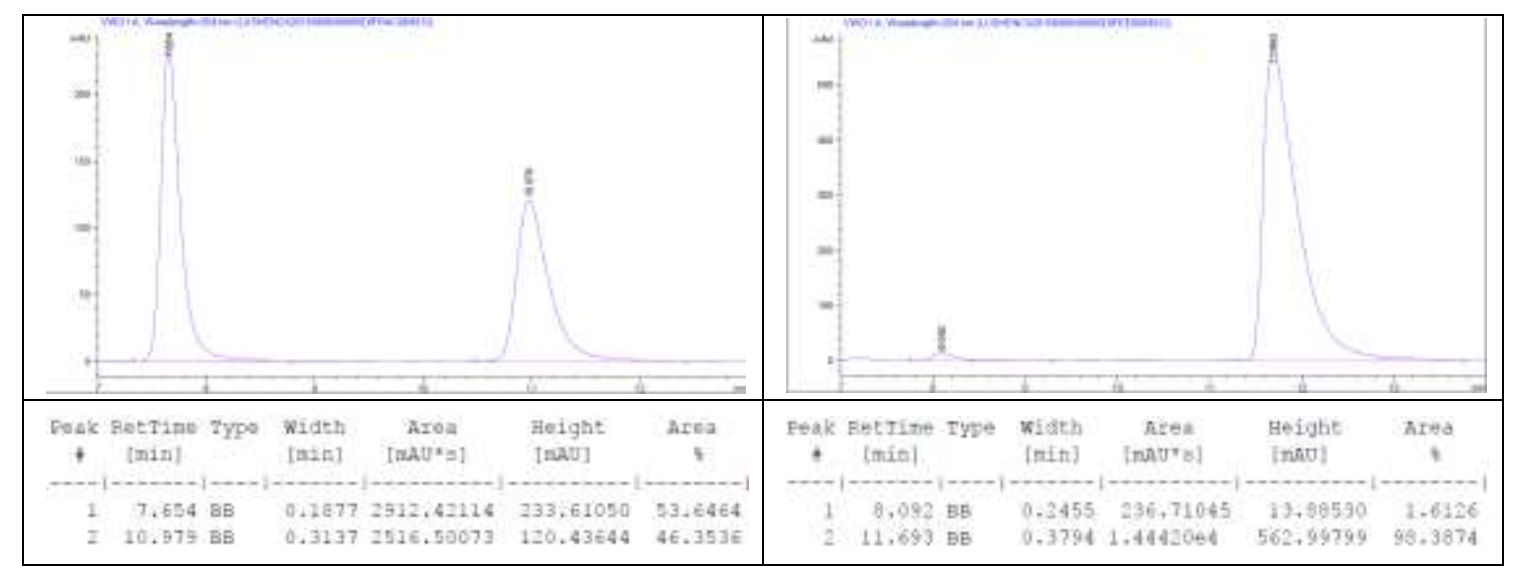

(S)-7-butyl-3,11-dichloro-7,8-dihydro-6H-benzo[2,3] oxocino[5,4-b]benzofuran-6-one.

(Scheme 4a, 4l)<smiles></smiles>

Used Enal 2c. White solid. MP: $100-101{ }^{\circ} \mathrm{C} .{ }^{1} \mathbf{H}$ NMR (500 MHz, $\left.\mathrm{CDCl}_{3}\right): \delta 7.49$ - 7.47 (m, 2H), $7.41(\mathrm{dd}, J=8.02 .0 \mathrm{~Hz}, 1 \mathrm{H}), 7.32(\mathrm{~d}, J=2.0 \mathrm{~Hz}, 1 \mathrm{H}), 7.29(\mathrm{~s}, 1 \mathrm{H}), 7.24(\mathrm{dd}, J=8.02 .0$ $\mathrm{Hz}, 1 \mathrm{H}), 3.26-3.13(\mathrm{~m}, 3 \mathrm{H}), 1.96-1.94(\mathrm{~m}, 1 \mathrm{H}), 1.59-1.56(\mathrm{~m}, 1 \mathrm{H}), 1.37-1.22(\mathrm{~m}, 4 \mathrm{H})$, $0.92(\mathrm{t}, J=7.0 \mathrm{~Hz}, 3 \mathrm{H}) .{ }^{13} \mathrm{C}$ NMR $\left(125 \mathrm{MHz}, \mathrm{CDCl}_{3}\right): \delta 173.00,154.41,152.21,149.77$, $134.96,132.04,130.70,127.18,126.89,123.97,122.89,122.32,119.63,111.73,111.34$, 38.03, 33.20, 32.66, 29.52, 22.43, 13.79. HRMS (ESI) m/z Calcd for $\left[\mathrm{C}_{21} \mathrm{H}_{18}{ }^{35} \mathrm{Cl}^{35} \mathrm{ClNaO}_{3}\right.$, $\mathrm{M}+\mathrm{Na}^{+}$: 411.0525; Found 411.0523. $\left[\mathrm{C}_{21} \mathrm{H}_{18}{ }^{35} \mathrm{Cl}^{37} \mathrm{ClNaO}_{3}, \mathrm{M}+\mathrm{Na}\right]^{+}$: Found 413.0493. $\left[\mathrm{C}_{21} \mathrm{H}_{18}{ }^{37} \mathrm{Cl}^{37} \mathrm{ClNaO}_{3}, \mathrm{M}+\mathrm{Na}\right]^{+}$: Found 415.0462 .

Optical Rotation: $[\alpha]^{25}{ }_{\mathrm{D}}-88.0\left(c=0.1, \mathrm{CHCl}_{3}\right) .97 \%$ ee (HPLC condition: Chiralpak ID column, $n$-Hexane $/ i-\mathrm{PrOH}=98: 2$, flow rate $=1.0 \mathrm{~mL} / \mathrm{min}$, wave length $=254 \mathrm{~nm}, t_{\mathrm{R}}=8.77$ $\min$ for minor isomer, $t_{\mathrm{R}}=13.57 \mathrm{~min}$ for major isomer). 


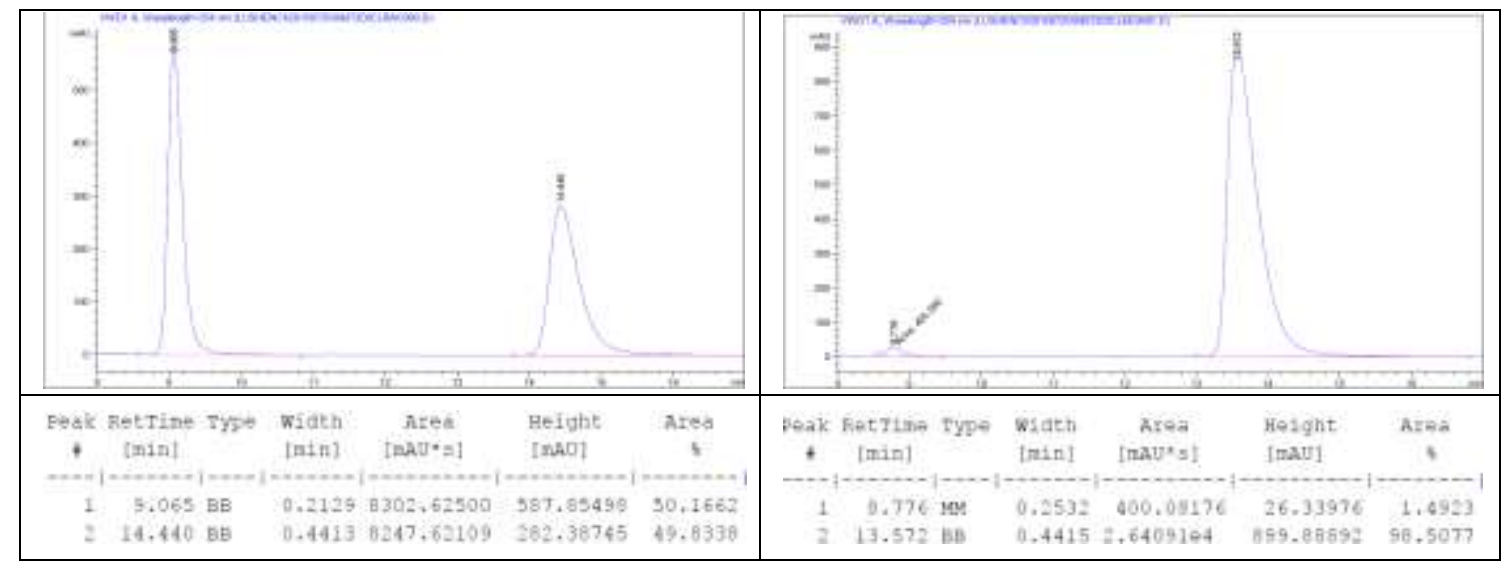

(S)-3,11-dibromo-7-butyl-7,8-dihydro-6H-benzo[2,3] oxocino[5,4-b]benzofuran-6-one.

(Scheme 4a, 4m)<smiles>O=C1Oc2cc(Br)ccc2-c2c(oc3cc(Br)ccc23)C[C@H]1Br</smiles>

Used Enal 2c. White solid. MP: $151-152{ }^{\circ} \mathrm{C} .{ }^{1} \mathbf{H}$ NMR (500 MHz, $\left.\mathrm{CDCl}_{3}\right): \delta 7.64(\mathrm{~d}, J=$ $1.5 \mathrm{~Hz}, 1 \mathrm{H}), 7.56(\mathrm{dd}, J=8.02 .0 \mathrm{~Hz}, 1 \mathrm{H}), 7.47(\mathrm{~d}, J=2.0 \mathrm{~Hz}, 1 \mathrm{H}), 7.41(\mathrm{~d}, J=8.0 \mathrm{~Hz}, 1 \mathrm{H})$, $7.38(\mathrm{dd}, J=8.01 .5 \mathrm{~Hz}, 1 \mathrm{H}), 7.26(\mathrm{~d}, J=8.5 \mathrm{~Hz}, 1 \mathrm{H}), 3.25-3.12(\mathrm{~m}, 3 \mathrm{H}), 1.96-1.92(\mathrm{~m}$, 1H), 1.59 - $1.54(\mathrm{~m}, 1 \mathrm{H}), 1.38-1.23(\mathrm{~m}, 4 \mathrm{H}), 0.92(\mathrm{t}, J=7.0 \mathrm{~Hz}, 3 \mathrm{H}) .{ }^{13} \mathbf{C} \mathbf{N M R}(125 \mathrm{MHz}$, $\left.\mathrm{CDCl}_{3}\right): \delta 172.95,154.66,152.11,149.82,132.29,129.82,127.53,126.67,125.20,123.35$, $122.55,120.04,118.08,114.64,111.44,38.02,33.18,32.66,29.51,22.43,13.78$. HRMS (ESI) $\mathrm{m} / \mathrm{z}$ Calcd for $\left[\mathrm{C}_{21} \mathrm{H}_{18}{ }^{79} \mathrm{Br}^{79} \mathrm{BrNaO}_{3}, \mathrm{M}+\mathrm{Na}\right]^{+}$: 498.9515 ; Found 498.9518. $\left[\mathrm{C}_{21} \mathrm{H}_{18}{ }^{79} \mathrm{Br}^{81} \mathrm{BrNaO}_{3}, \mathrm{M}+\mathrm{Na}\right]^{+}$: Found 500.9498. $\left[\mathrm{C}_{21} \mathrm{H}_{18}{ }^{81} \mathrm{Br}^{81} \mathrm{BrNaO}_{3}, \mathrm{M}+\mathrm{Na}\right]^{+}$: Found 502.9475 .

Optical Rotation: $[\alpha]^{25}{ }_{\mathrm{D}}-70.0\left(c=0.1, \mathrm{CHCl}_{3}\right) .97 \%$ ee (HPLC condition: Chiralpak ID column, $n$-Hexane $/ i-\mathrm{PrOH}=98: 2$, flow rate $=1.0 \mathrm{~mL} / \mathrm{min}$, wave length $=254 \mathrm{~nm}, t_{\mathrm{R}}=10.49$ $\min$ for minor isomer, $t_{\mathrm{R}}=17.96 \mathrm{~min}$ for major isomer).

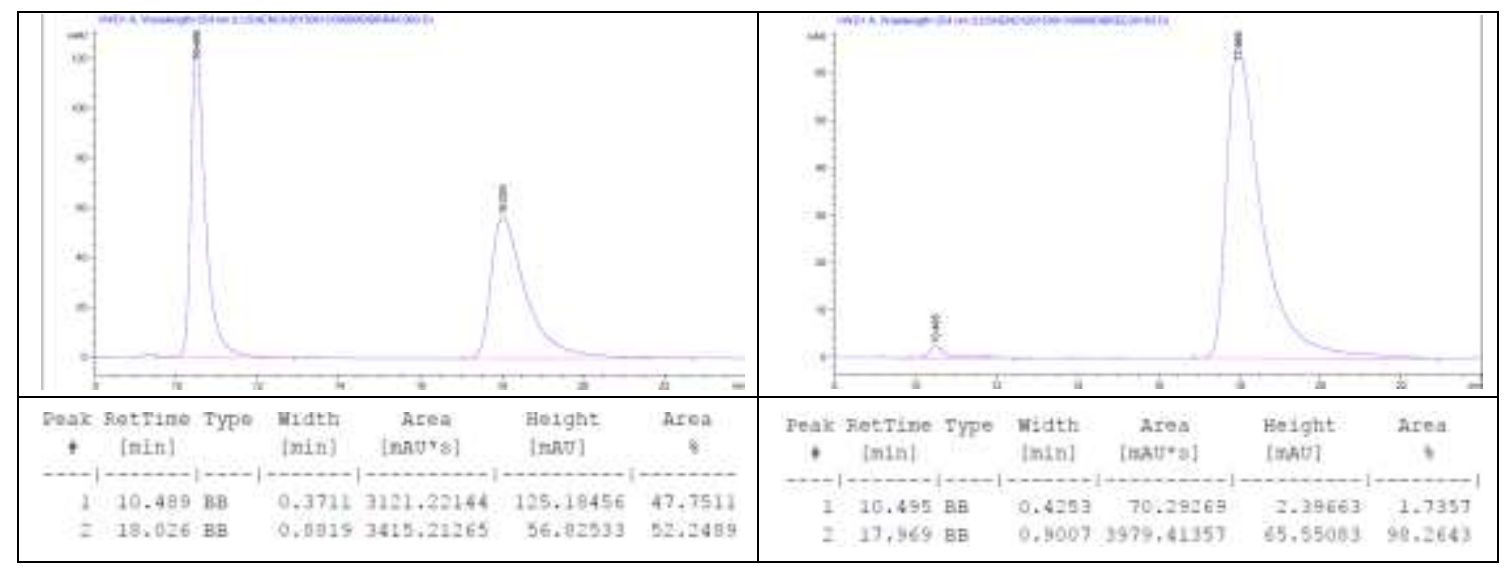


(S)-7-butyl-2,12-dimethyl-7,8-dihydro-6H-benzo[2,3] oxocino[5,4-b]benzofuran-6-one.

(Scheme 4a, 4n)<smiles>Cc1ccc2c(c1)-c1c(oc3ccc(C)cc13)CC(Br)C(=O)O2</smiles>

Used Enal 2c. Syrup. ${ }^{1} \mathbf{H}$ NMR (500 MHz, $\left.\mathrm{CDCl}_{3}\right): \delta 7.39$ (s, 1H), $7.33(\mathrm{~d}, J=8.5 \mathrm{~Hz}, 1 \mathrm{H})$, $7.25-7.23(\mathrm{~m}, 2 \mathrm{H}), 7.15(\mathrm{~d}, J=8.5 \mathrm{~Hz}, 1 \mathrm{H}), 7.12(\mathrm{~d}, J=8.5 \mathrm{~Hz}, 1 \mathrm{H}), 3.29-3.09(\mathrm{~m}, 3 \mathrm{H})$, $2.47(\mathrm{~s}, 3 \mathrm{H}), 2.44(\mathrm{~s}, 3 \mathrm{H}), 1.97-1.91(\mathrm{~m}, 1 \mathrm{H}), 1.56-1.49(\mathrm{~m}, 1 \mathrm{H}), 1.38-1.20(\mathrm{~m}, 4 \mathrm{H}), 0.90$ $(\mathrm{t}, J=7.0 \mathrm{~Hz}, 3 \mathrm{H}) .{ }^{13} \mathbf{C}$ NMR $\left(125 \mathrm{MHz}, \mathrm{CDCl}_{3}\right): \delta 174.19,152.83,151.31,147.27,136.12$, $132.48,131.77,129.90,128.86,125.72,124.59,121.38,119.11,111.95,110.46,37.73,33.45$, 32.64, 29.57, 22.37, 21.34, 20.90, 13.81. HRMS (ESI) $\mathrm{m} / \mathrm{z}$ Calcd for $\left[\mathrm{C}_{23} \mathrm{H}_{24} \mathrm{NaO}_{3}, \mathrm{M}+\mathrm{Na}\right]^{+}$: 371.1617; Found 371.1619.

Optical Rotation: $[\alpha]^{25}{ }_{\mathrm{D}}-81.0\left(c=0.1, \mathrm{CHCl}_{3}\right) .99 \%$ ee (HPLC condition: Chiralpak ID column, $n$-Hexane $/ i-\mathrm{PrOH}=98: 2$, flow rate $=1.0 \mathrm{~mL} / \mathrm{min}$, wave length $=254 \mathrm{~nm}, t_{\mathrm{R}}=11.17$ $\min$ for minor isomer, $t_{\mathrm{R}}=22.66 \mathrm{~min}$ for major isomer).

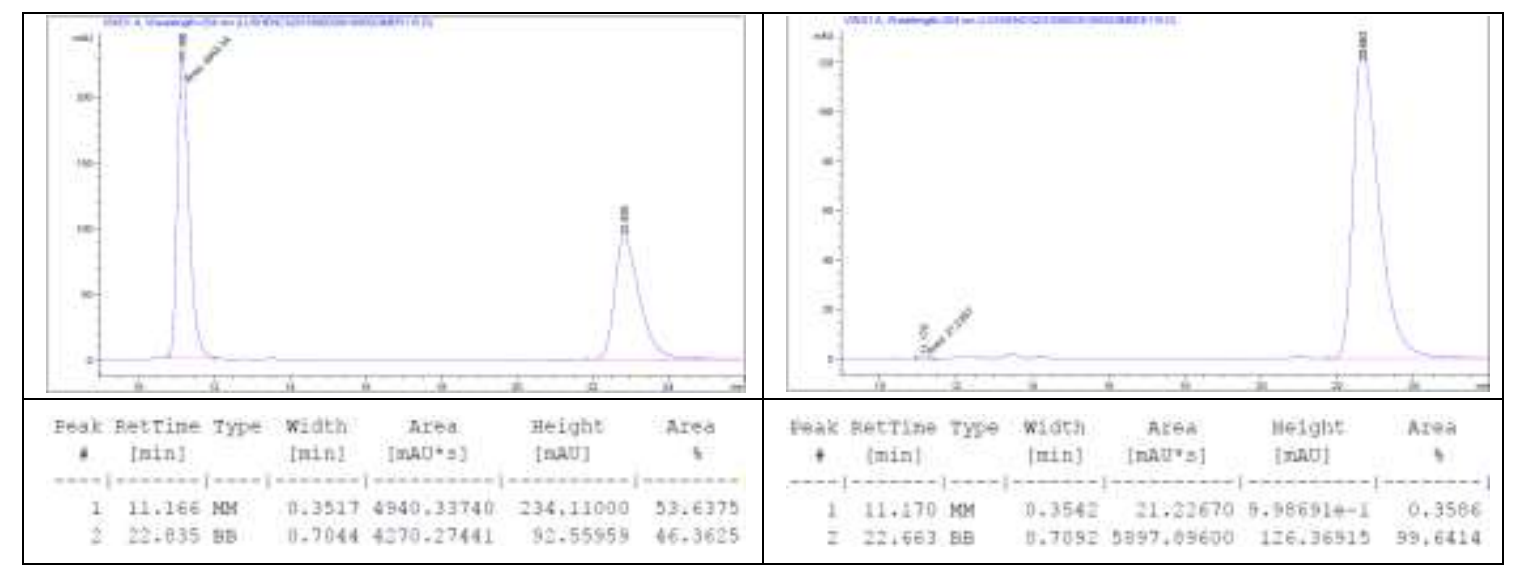

(S)-2,12-di-tert-butyl-7-butyl-7,8-dihydro-6H-benzo[2,3] oxocino[5,4-b]benzofuran-6-one .

(Scheme 4a, 4o)<smiles>CC(C)(C)c1ccc2oc3c(c2c1)-c1c(cccc1C(C)(C)C)OC(=O)C(Br)C3</smiles>

Used Enal 2c. Oil. ${ }^{1} \mathbf{H}$ NMR $\left(500 \mathrm{MHz}, \mathrm{CDCl}_{3}\right): \delta 7.65(\mathrm{~d}, J=2.0 \mathrm{~Hz}, 1 \mathrm{H}), 7.46-7.44(\mathrm{~m}$, 2H), $7.39-7.38(\mathrm{~m}, 2 \mathrm{H}), 7.21(\mathrm{dd}, J=8.52 .5 \mathrm{~Hz}, 1 \mathrm{H}), 3.35-3.12(\mathrm{~m}, 3 \mathrm{H}), 1.98-1.94(\mathrm{~m}$, 1H), 1.59 - 1.52 (m, 1H), 1.42 (s, 9H), $1.36(\mathrm{~s}, 9 \mathrm{H}), 1.36-1.21(\mathrm{~m}, 4 \mathrm{H}), 0.91$ (t, $J=7.0 \mathrm{~Hz}$, $3 \mathrm{H}) .{ }^{13} \mathrm{C}$ NMR $\left(125 \mathrm{MHz}, \mathrm{CDCl}_{3}\right): \delta 174.27,152.67,151.28,149.27,147.01,146.16,128.79$, $128.56,126.09,124.05,122.39,121.05,115.54,112.68,110.32,37.79,34.76,34.69,33.49$, $32.77,31.79,31.41,29.62,22.47,13.87$. HRMS (ESI) $\mathrm{m} / \mathrm{z}$ Calcd for $\left[\mathrm{C}_{29} \mathrm{H}_{36} \mathrm{NaO}_{3}, \mathrm{M}+\mathrm{Na}\right]^{+}$: 455.2556; Found 455.2552. 
Optical Rotation: $[\alpha]^{25} \mathrm{D}-16.0\left(c=0.3, \mathrm{CHCl}_{3}\right) .99 \%$ ee (HPLC condition: Chiralpak ID column, $n$-Hexane $/ i-\mathrm{PrOH}=98: 2$, flow rate $=1.0 \mathrm{~mL} / \mathrm{min}$, wave length $=254 \mathrm{~nm}, t_{\mathrm{R}}=6.09$ $\min$ for minor isomer, $t_{\mathrm{R}}=8.81 \mathrm{~min}$ for major isomer).

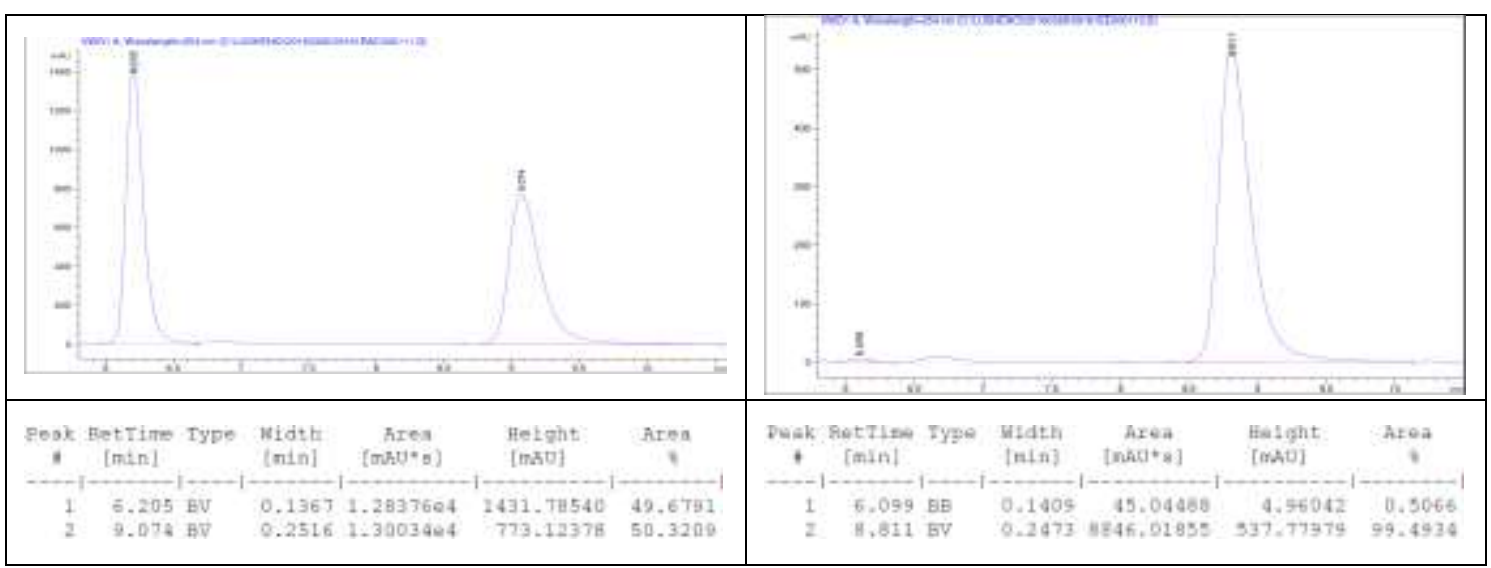

(S)-2,12-dibromo-7-butyl-7,8-dihydro-6H-benzo[2,3]oxocino[5,4- $b]$ benzofuran-6-one.

(Scheme 4a, 4p)<smiles>O=C1Oc2ccc(Br)cc2-c2c(oc3ccc(Br)cc23)CC1[18O]</smiles>

Used Enal 2c. Syrup. ${ }^{1} \mathbf{H}$ NMR $\left(500 \mathrm{MHz}, \mathrm{CDCl}_{3}\right): \delta 7.69(\mathrm{~d}, J=2.0 \mathrm{~Hz}, 1 \mathrm{H}), 7.59(\mathrm{dd}, J=$ $9.02 .0 \mathrm{~Hz}, 1 \mathrm{H}), 7.57(\mathrm{~d}, J=2.0 \mathrm{~Hz}, 1 \mathrm{H}), 7.44(\mathrm{dd}, J=9.01 .5 \mathrm{~Hz}, 1 \mathrm{H}), 7.34(\mathrm{~d}, J=8.5 \mathrm{~Hz}$, $1 \mathrm{H}), 7.17(\mathrm{~d}, J=9.0 \mathrm{~Hz}, 1 \mathrm{H}), 3.26-3.13(\mathrm{~m}, 3 \mathrm{H}), 1.98-1.92(\mathrm{~m}, 1 \mathrm{H}), 1.56-1.52(\mathrm{~m}, 1 \mathrm{H})$, $1.37-1.20(\mathrm{~m}, 4 \mathrm{H}), 0.90(\mathrm{t}, J=7.0 \mathrm{~Hz}, 3 \mathrm{H}) .{ }^{13} \mathbf{C}$ NMR $\left(125 \mathrm{MHz}, \mathrm{CDCl}_{3}\right): \delta 173.05,153.19$, $153.14,148.50,133.83,132.78,130.25,127.93,126.30,123.51,121.77,119.68,116.57$, 112.63, 110.81, 37.82, 33.27, 32.58, 29.52, 22.35, 13.79. HRMS (ESI) $\mathrm{m} / \mathrm{z}$ Calcd for $\left[\mathrm{C}_{21} \mathrm{H}_{18}{ }^{79} \mathrm{Br}^{79} \mathrm{BrNaO}_{3}, \mathrm{M}+\mathrm{Na}\right]^{+}:$498.9515; Found 498.9517. $\left[\mathrm{C}_{21} \mathrm{H}_{18}{ }^{79} \mathrm{Br}^{81} \mathrm{BrNaO}_{3}, \mathrm{M}+\mathrm{Na}\right]^{+}$: Found 500.9496. $\left[\mathrm{C}_{21} \mathrm{H}_{18}{ }^{81} \mathrm{Br}^{81} \mathrm{BrNaO}_{3}, \mathrm{M}+\mathrm{Na}\right]^{+}$: Found 502.9470.

Optical Rotation: $[\alpha]^{25} \mathrm{D}-63.0\left(c=0.1, \mathrm{CHCl}_{3}\right) .98 \%$ ee (HPLC condition: Chiralpak ID column, $n$-Hexane $/ i-\mathrm{PrOH}=98: 2$, flow rate $=1.0 \mathrm{~mL} / \mathrm{min}$, wave length $=254 \mathrm{~nm}, t_{\mathrm{R}}=9.62$ $\min$ for minor isomer, $t_{\mathrm{R}}=11.33 \mathrm{~min}$ for major isomer).

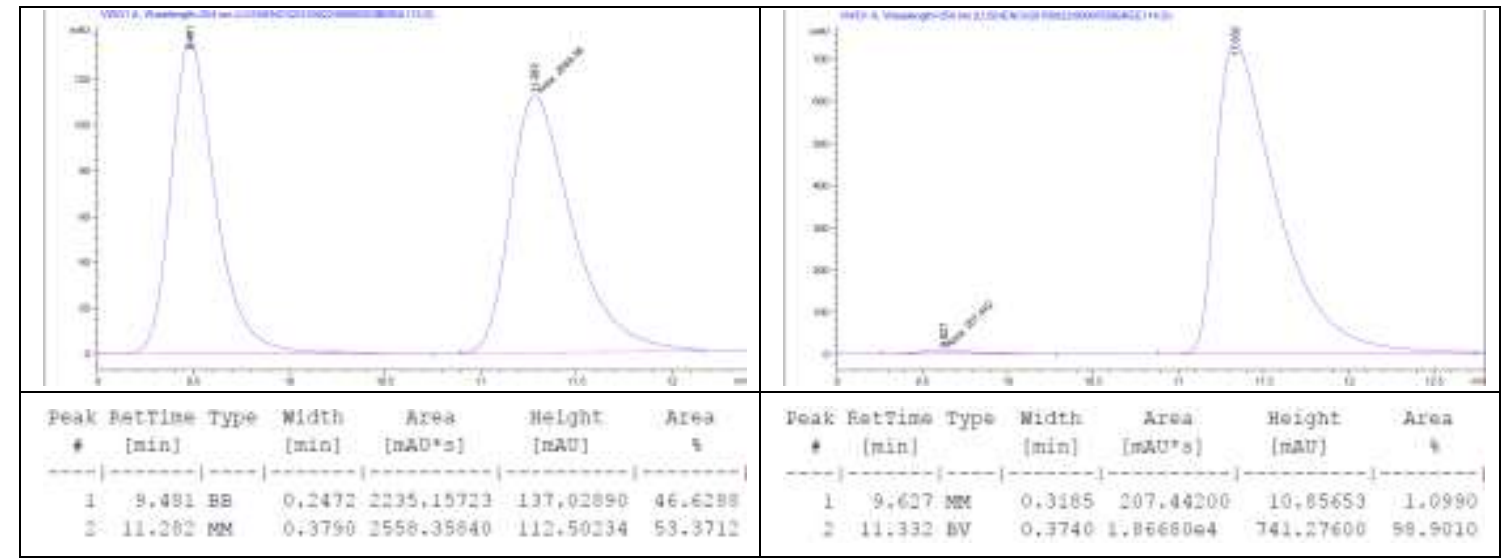


(7S)-7-butyl-8-methyl-7,8-dihydro-6H-benzo[2,3]oxocino[5,4-b]benzofuran-6-one. (Scheme $4 \mathrm{a}, \mathbf{4 q})$

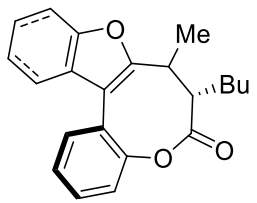

Used Enal 2c. ${ }^{1} \mathbf{H}$ NMR (500 MHz, $\left.\mathrm{CDCl}_{3}\right): \delta 7.63(\mathrm{dd}, J=7.51 .5 \mathrm{~Hz}, 0.3 \mathrm{H}), 7.60(\mathrm{dd}, J=$ $7.51 .0 \mathrm{~Hz}, 1 \mathrm{H}), 7.49-7.46(\mathrm{~m}, 2.5 \mathrm{H}), 7.44-7.39(\mathrm{~m}, 2.5 \mathrm{H}), 7.33-7.31(\mathrm{~m}, 1.3 \mathrm{H}), 7.27-$ $7.22(\mathrm{~m}, 2.5 \mathrm{H}), 3.55-3.53(\mathrm{~m}, 0.3 \mathrm{H}), 3.50-3.40(\mathrm{~m}, 0.3 \mathrm{H}), 3.30-3.27(\mathrm{~m}, 1 \mathrm{H}), 2.97-2.92$ $(\mathrm{m}, 1 \mathrm{H}), 2.10-2.00(\mathrm{~m}, 0.4 \mathrm{H}), 1.87-1.82(\mathrm{~m}, 1 \mathrm{H}), 1.72-1.65(\mathrm{~m}, 1.4 \mathrm{H}), 1.47-1.40(\mathrm{~m}$, 1.5H), $1.36-1.24(\mathrm{~m}, 7.3 \mathrm{H}), 1.21-1.14(\mathrm{~m}, 1.4 \mathrm{H}), 0.92-0.87(\mathrm{~m}, 4.3 \mathrm{H}) .{ }^{13} \mathbf{C}$ NMR $(125$ $\left.\mathrm{MHz}, \mathrm{CDCl}_{3}\right): \delta 173.72,171.84,156.99,154.87,154.38,154.11,149.62,149.52,131.34$, $131.30,129.50,129.19,128.98,128.83,126.32,126.21,125.73,124.68,124.62,124.55$, $122.98,122.95,121.80,121.24,119.58,119.39,111.26,111.07,110.97,110.60,44.78,40.92$, $38.41,36.73,30.73,30.05,29.85,29.68,29.64,22.43,22.40,19.04,13.81,11.78$. HRMS (ESI) $\mathrm{m} / \mathrm{z}$ Calcd for $\left[\mathrm{C}_{22} \mathrm{H}_{22} \mathrm{NaO}_{3}, \mathrm{M}+\mathrm{Na}\right]^{+}: 357.1461$; Found 357.1464 .

97\% ee for major product and 99\% ee for minor product. (HPLC condition: Chiralpak ID column, $n$-Hexane $/ i-\mathrm{PrOH}=99: 1$, flow rate $=1.0 \mathrm{~mL} / \mathrm{min}$, wave length $=254 \mathrm{~nm}, t_{\mathrm{R}}=10.93$ $\min$ for minor isomer, $t_{\mathrm{R}}=21.13 \mathrm{~min}$ for major isomer and $t_{\mathrm{R}}=8.10 \mathrm{~min}$ for minor isomer, $t_{\mathrm{R}}$ $=10.29 \mathrm{~min}$ for major isomer).

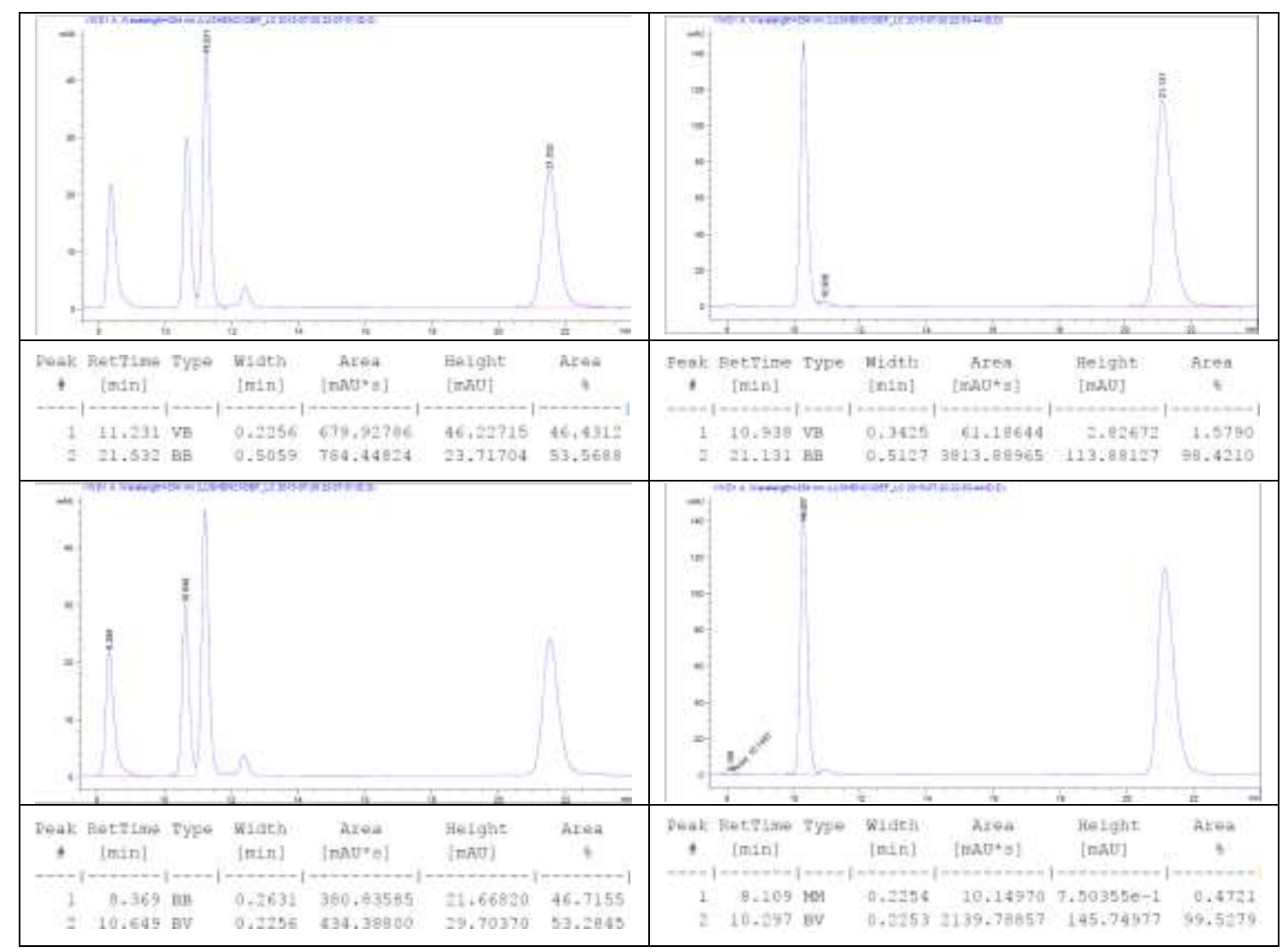


(7R,8S)-7-isopropyl-8-methyl-7,8-dihydro-6H-benzo[2,3] oxocino[5,4-b]benzofuran-6-one.

(Scheme 4a, 4r)

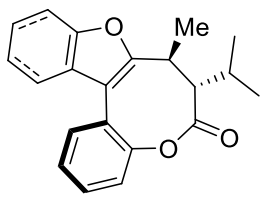

Used 2-Benzoylisovaleraldehyde 2a. White solid, MP: 167 - $168{ }^{\circ} \mathrm{C} .{ }^{1} \mathbf{H}$ NMR (300 MHz, $\left.\mathrm{CDCl}_{3}\right): \delta 7.58(\mathrm{~d}, J=7.5 \mathrm{~Hz}, 1 \mathrm{H}), 7.48-7.45(\mathrm{~m}, 2 \mathrm{H}), 7.40-7.38(\mathrm{~m}, 2 \mathrm{H}), 7.32(\mathrm{t}, J=7.5$ $\mathrm{Hz}, 1 \mathrm{H}), 7.26-7.22(\mathrm{~m}, 2 \mathrm{H}), 3.57-3.50(\mathrm{~m}, 1 \mathrm{H}), 3.15(\mathrm{dd}, J=12.04 .5 \mathrm{~Hz}, 1 \mathrm{H}), 2.08-2.00$ $(\mathrm{m}, 1 \mathrm{H}), 1.30(\mathrm{~d}, J=7.0 \mathrm{~Hz}, 3 \mathrm{H}), 1.24(\mathrm{~d}, J=7.0 \mathrm{~Hz}, 3 \mathrm{H}), 1.04(\mathrm{~d}, J=7.0 \mathrm{~Hz}, 3 \mathrm{H}) .{ }^{13} \mathbf{C}$ NMR $\left(125 \mathrm{MHz}, \mathrm{CDCl}_{3}\right): \delta 172.08,157.20,154.32,149.94,131.38,129.56,129.09,125.96$, 124.64, 124.63, 122.96, 121.10, 119.48, 111.03, 110.85, 48.00, 35.63, 27.79, 20.96, 18.43, 15.28. HRMS (ESI) $\mathrm{m} / \mathrm{z}$ Calcd for $\left[\mathrm{C}_{21} \mathrm{H}_{20} \mathrm{NaO}_{3}, \mathrm{M}+\mathrm{Na}^{+}\right.$: 343.1304 ; Found 343.1306.

Optical Rotation: $[\alpha]^{25} \mathrm{D}-62.0\left(c=0.1, \mathrm{CHCl}_{3}\right) .99 \%$ ee (HPLC condition: Chiralpak ID column, $n$-Hexane $/ i-\mathrm{PrOH}=99: 1$, flow rate $=1.0 \mathrm{~mL} / \mathrm{min}$, wave length $=254 \mathrm{~nm}, t_{\mathrm{R}}=$ $11.78 \mathrm{~min}$ for minor isomer, $t_{\mathrm{R}}=20.40 \mathrm{~min}$ for major isomer).

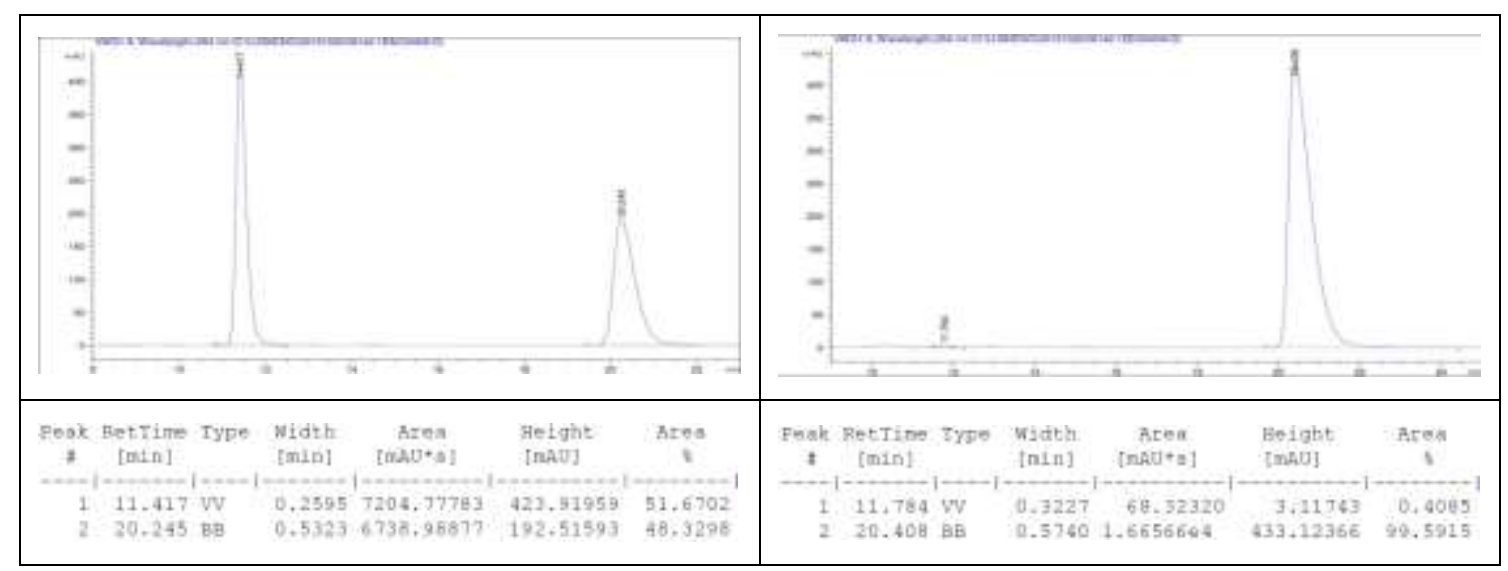

(7R,8R)-7-isopropyl-8-methyl-7,8-dihydro-6H-benzo[2,3] oxocino[5,4-b]benzofuran-6-one. (Scheme 4a, 4r')<smiles>CC(C)[C@H]1C(=O)Oc2ccccc2-c2c1oc1ccccc21</smiles>

Used 2-Benzoylisovaleraldehyde 2a. White solid, MP: 123 - $124{ }^{\circ} \mathrm{C} .{ }^{1} \mathbf{H}$ NMR $(500 \mathrm{MHz}$, Chloroform- $d$ ) $\delta 7.59$ (dd, $J=7.3,2.1 \mathrm{~Hz}, 1 \mathrm{H}), 7.49-7.37(\mathrm{~m}, 4 \mathrm{H}), 7.30(\mathrm{t}, J=7.9 \mathrm{~Hz}, 1 \mathrm{H})$, $7.26-7.21(\mathrm{~m}, 2 \mathrm{H}), 3.58(\mathrm{qd}, J=7.0,4.3 \mathrm{~Hz}, 1 \mathrm{H}), 3.09(\mathrm{dd}, J=10.7,4.5 \mathrm{~Hz}, 1 \mathrm{H}), 2.35-$ $2.27(\mathrm{~m}, 1 \mathrm{H}), 1.60(\mathrm{~d}, J=7.5 \mathrm{~Hz}, 3 \mathrm{H}), 0.92(\mathrm{~d}, J=3.0 \mathrm{~Hz}, 3 \mathrm{H}), 0.91(\mathrm{~d}, J=3.0 \mathrm{~Hz}, 3 \mathrm{H}) .{ }^{13} \mathbf{C}$ NMR $\left(125 \mathrm{MHz}, \mathrm{CDCl}_{3}\right): \delta 171.80,155.09,154.13,149.58,131.42,129.26,128.91,126.24$, $125.65,124.53,122.95,121.54,119.55,113.96,110.94,48.47,33.88,27.75,21.85,18.81$, 10.94. HRMS (ESI) $\mathrm{m} / \mathrm{z}$ Calcd for $\left[\mathrm{C}_{21} \mathrm{H}_{20} \mathrm{NaO}_{3}, \mathrm{M}+\mathrm{Na}\right]^{+}: 343.1304$; Found 343.1302. 
Optical Rotation: $[\alpha]^{25}$ - $24.0\left(c=0.1, \mathrm{CHCl}_{3}\right) .97 \%$ ee (HPLC condition: Chiralpak ID column, $n$-Hexane $/ i-\mathrm{PrOH}=99: 1$, flow rate $=1.0 \mathrm{~mL} / \mathrm{min}$, wave length $=254 \mathrm{~nm}, t_{\mathrm{R}}=$ $10.31 \mathrm{~min}$ for minor isomer, $t_{\mathrm{R}}=13.23 \mathrm{~min}$ for major isomer).

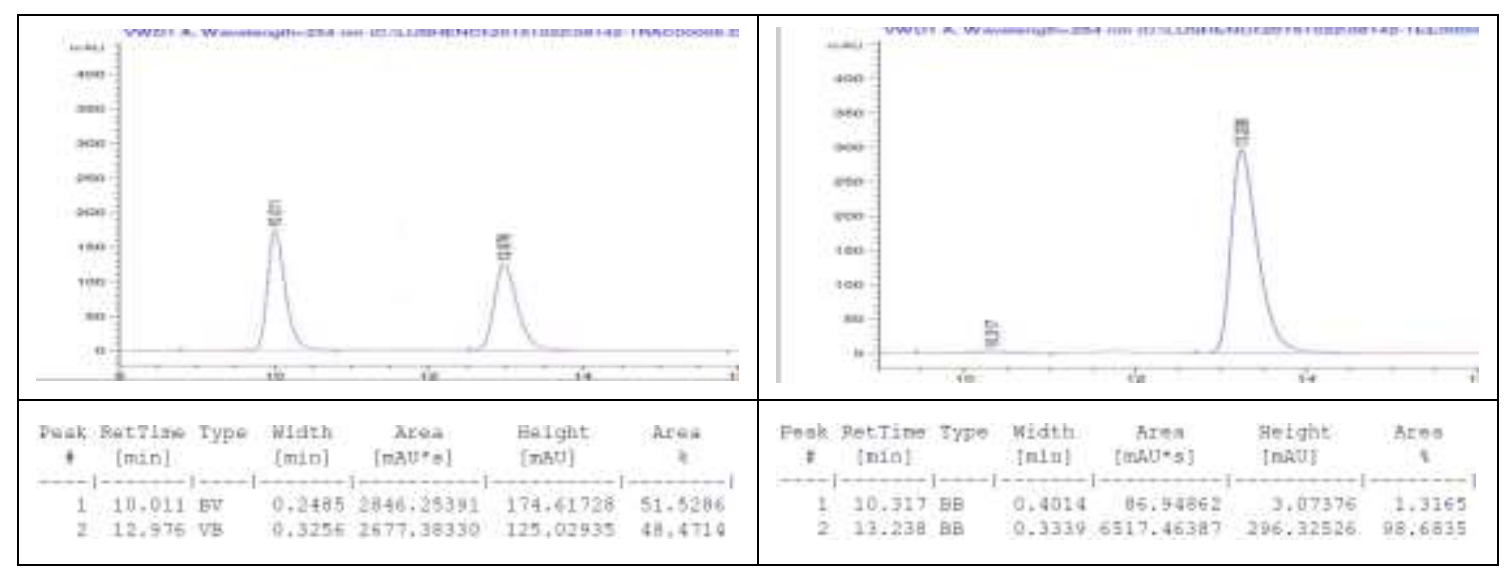

(S)-7-butyl-3-methoxy-7,8-dihydro-6H-benzo[2,3] oxocino[5,4-b]benzofuran-6-one. (Scheme $4 \mathrm{~b}, 4 \mathrm{~s})$<smiles>COc1ccc2c(c1)OC(=O)C([18O])Cc1oc3ccccc3c1-2</smiles>

Used Enal 2c. Syrup. ${ }^{1} \mathbf{H}$ NMR (500 MHz, $\left.\mathrm{CDCl}_{3}\right): \delta 7.50(\mathrm{~d}, J=8.5 \mathrm{~Hz}, 1 \mathrm{H}), 7.45(\mathrm{~d}, J=8.5$ $\mathrm{Hz}, 1 \mathrm{H}), 7.43(\mathrm{~d}, J=8.0 \mathrm{~Hz}, 1 \mathrm{H}), 7.32-7.29(\mathrm{~m}, 1 \mathrm{H}), 7.24(\mathrm{t}, J=7.5 \mathrm{~Hz}, 1 \mathrm{H}), 6.98$ (dd, $J=$ $8.52 .5 \mathrm{~Hz}, 1 \mathrm{H}), 6.83(\mathrm{~d}, J=2.5 \mathrm{~Hz}, 1 \mathrm{H}), 3.91(\mathrm{~s}, 3 \mathrm{H}), 3.29-3.11(\mathrm{~m}, 3 \mathrm{H}), 1.97-1.93(\mathrm{~m}$, 1H), $1.59-1.54(\mathrm{~m}, 1 \mathrm{H}), 1.38-1.22(\mathrm{~m}, 4 \mathrm{H}), 0.91(\mathrm{t}, J=7.0 \mathrm{~Hz}, 3 \mathrm{H}) .{ }^{13} \mathbf{C} \mathbf{N M R}(125 \mathrm{MHz}$, $\left.\mathrm{CDCl}_{3}\right): \delta 173.85,160.44,154.36,150.88,150.17,131.95,129.03,124.48,122.91,119.28$, 116.77, 112.51, 111.91, 110.94, 107.15, 55.68, 38.02, 33.30, 32.71, 29.56, 22.40, 13.81. HRMS (ESI) m/z Calcd for $\left[\mathrm{C}_{22} \mathrm{H}_{22} \mathrm{NaO}_{4}, \mathrm{M}+\mathrm{Na}\right]^{+}:$373.1410; Found 373.1414.

Optical Rotation: $[\alpha]^{25}{ }_{\mathrm{D}}-74.0\left(c=0.1, \mathrm{CHCl}_{3}\right) .99 \%$ ee (HPLC condition: Chiralpak ID column, $n$-Hexane $/ i-\mathrm{PrOH}=80: 20$, flow rate $=1.0 \mathrm{~mL} / \mathrm{min}$, wave length $=254 \mathrm{~nm}, t_{\mathrm{R}}=7.81$ $\mathrm{min}$ for minor isomer, $t_{\mathrm{R}}=16.90 \mathrm{~min}$ for major isomer).

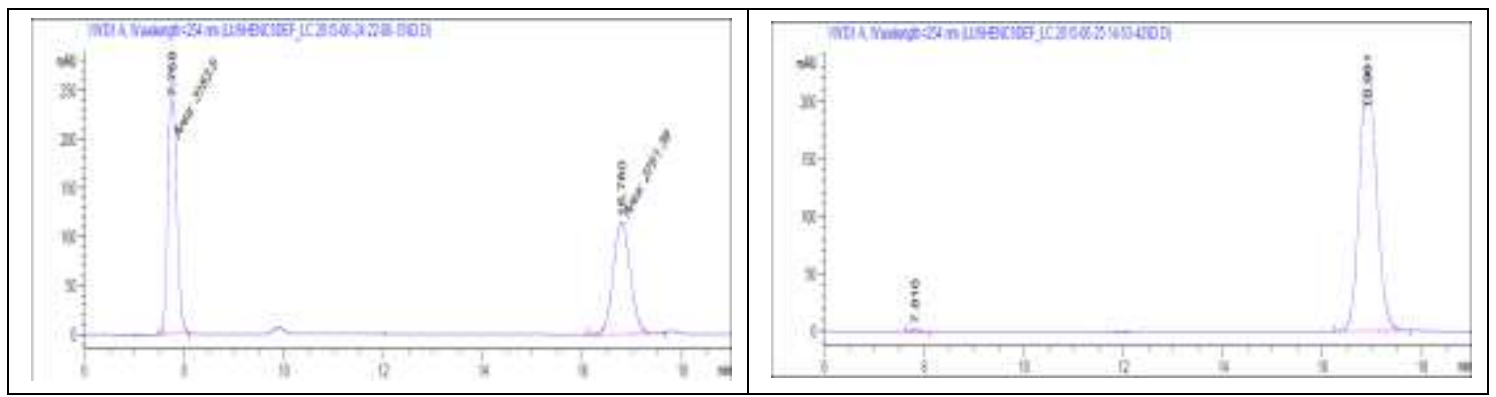




\begin{tabular}{|c|c|c|c|c|c|c|c|c|c|c|c|}
\hline $\begin{array}{c}\text { Peak } \\
\vdots \\
\ldots\end{array}$ & $\begin{array}{c}\text { RetTime } \\
\text { [min] }\end{array}$ & Type: & $\begin{array}{l}\text { Width } \\
\text { (min) }\end{array}$ & $\begin{array}{c}\text { Arcea } \\
{[\mathrm{m} A J * 5]}\end{array}$ & $\begin{array}{c}\text { Area } \\
+ \\
\end{array}$ & $\begin{array}{c}\text { Peak } \\
\neq\end{array}$ & $\begin{array}{c}\text { RetTine } \\
\text { [min] }\end{array}$ & Type & $\begin{array}{l}\text { Width } \\
\text { [min] }\end{array}$ & $\begin{array}{c}\text { Area } \\
{\left[m A U^{*} s\right]}\end{array}$ & $\begin{array}{c}\text { Area } \\
\&\end{array}$ \\
\hline $\begin{array}{l}1 \\
2\end{array}$ & $\begin{array}{r}7.756 \\
16.780\end{array}$ & $\mathrm{MM}$ & $\begin{array}{l}0.1931 \\
0.4004\end{array}$ & $\begin{array}{l}3173.50244 \\
2751.38989\end{array}$ & $\begin{array}{l}53.6404 \\
46.3596\end{array}$ & $\begin{array}{l}1 \\
2\end{array}$ & $\begin{array}{r}7.816 \\
16.901\end{array}$ & $\begin{array}{l}\text { BB } \\
\text { BEB }\end{array}$ & $\begin{array}{l}0.1415 \\
0.3695\end{array}$ & $\begin{array}{r}22.82956 \\
5440.45850\end{array}$ & $\begin{array}{r}0.4179 \\
99.5821\end{array}$ \\
\hline
\end{tabular}

(S)-7-butyl-12-nitro-7,8-dihydro-6H-benzo[2,3] oxocino[5,4-b]benzofuran-6-one. (Scheme 4b,

4t)<smiles>COc1ccccc1-c1c(CC(C)C)oc2ccc([N+](=O)[O-])cc12</smiles>

Used Enal 2c. Syrup. ${ }^{1} \mathbf{H}$ NMR $\left(500 \mathrm{MHz}\right.$, acetone - $\left.\mathrm{d}_{6}\right): \delta 8.31(\mathrm{~d}, J=2.5 \mathrm{~Hz}, 1 \mathrm{H}), 8.28(\mathrm{dd}$, $J=9.02 .5 \mathrm{~Hz}, 1 \mathrm{H}), 7.77(\mathrm{~d}, J=9.0 \mathrm{~Hz}, 1 \mathrm{H}), 7.73(\mathrm{dd}, J=7.52 .0 \mathrm{~Hz}, 1 \mathrm{H}), 7.61(\mathrm{td}, J=8.0$ $2.0 \mathrm{~Hz}, 1 \mathrm{H}), 7.56(\mathrm{td}, J=7.51 .5 \mathrm{~Hz}, 1 \mathrm{H}), 7.41(\mathrm{dd}, J=8.01 .0 \mathrm{~Hz}, 1 \mathrm{H}), 3.48$ (dd, $J=17.04 .5$ $\mathrm{Hz}, 1 \mathrm{H}), 3.33-3.30(\mathrm{~m}, 1 \mathrm{H}), 3.13(\mathrm{dd}, J=17.013 .0 \mathrm{~Hz}, 1 \mathrm{H}), 1.89-1.86(\mathrm{~m}, 1 \mathrm{H}), 1.63-1.59$ $(\mathrm{m}, 1 \mathrm{H}), 1.31-1.22(\mathrm{~m}, 4 \mathrm{H}), 0.86(\mathrm{t}, J=7.0 \mathrm{~Hz}, 3 \mathrm{H}) .{ }^{13} \mathbf{C}$ NMR $\left(125 \mathrm{MHz}\right.$, acetone $\left.-\mathrm{d}_{6}\right): \delta$ $172.90,157.14,155.60,149.51,144.66,131.42,130.53,129.32,127.01,123.16,121.97$, $120.53,115.50,112.90,111.81,37.62,33.04,32.21,29.28,22.07,13.15$. HRMS (ESI) m/z Calcd for $\left[\mathrm{C}_{21} \mathrm{H}_{19} \mathrm{NNaO}_{5}, \mathrm{M}+\mathrm{Na}\right]^{+}$: 388.1155; Found 388.1157.

Optical Rotation: $[\alpha]^{25} \mathrm{D}-45.0\left(c=0.1, \mathrm{CHCl}_{3}\right) .98 \%$ ee (HPLC condition: Chiralpak ID column, $n$-Hexane $/ i$-PrOH $=80: 20$, flow rate $=1.0 \mathrm{~mL} / \mathrm{min}$, wave length $=254 \mathrm{~nm}, t_{\mathrm{R}}=$ $12.41 \mathrm{~min}$ for minor isomer, $t_{\mathrm{R}}=20.48 \mathrm{~min}$ for major isomer).

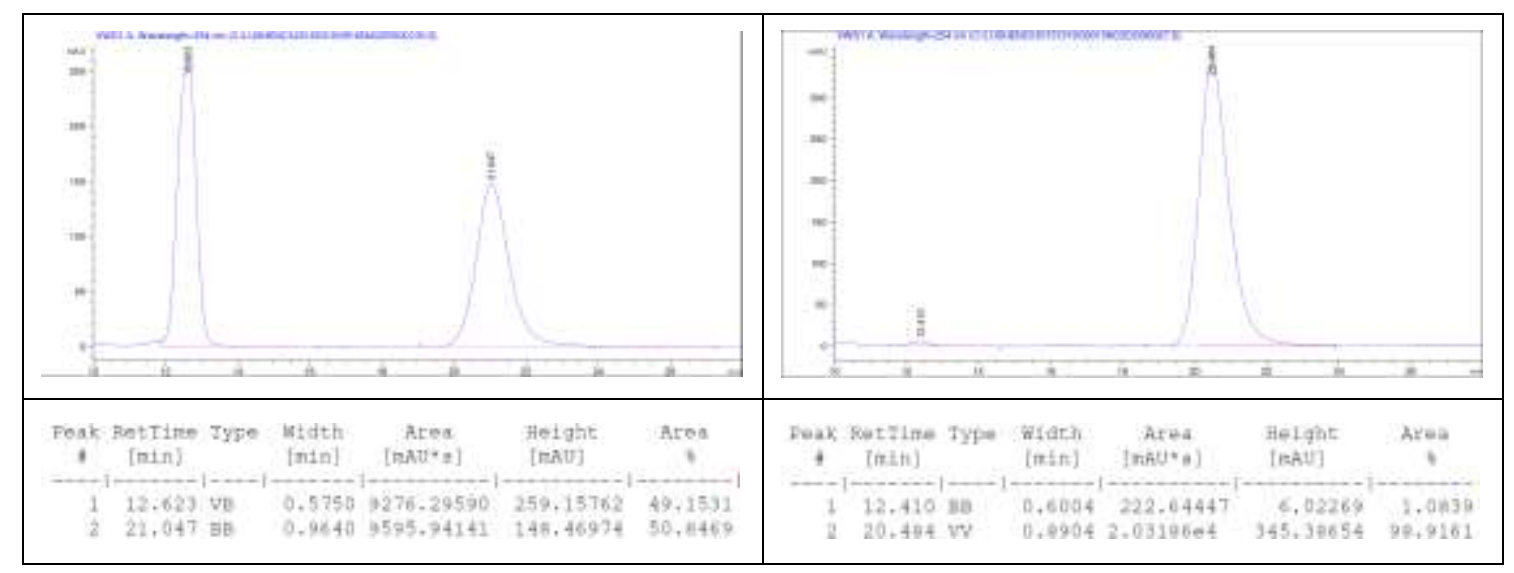




\section{The synthesis of indole-derived bridged biaryls.}

\subsection{Optimization studies.}

Table S2.

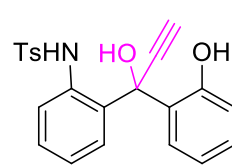

$5 a$

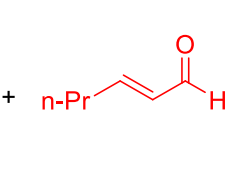

2c

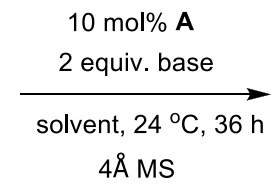

$4 \AA \mathrm{MS}$

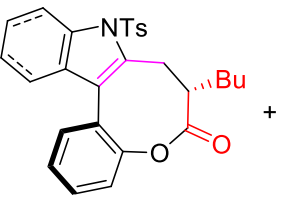

$6 a$

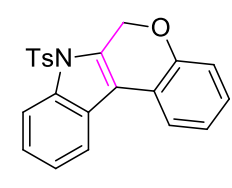

8

\begin{tabular}{|c|c|c|c|c|c|c|}
\hline Entry & Cat. & Solvent & Base & 6a Yield $(\%)^{*}$ & 6a e.e. $(\%)^{\dagger}$ & $\begin{array}{c}\mathbf{8} \\
\text { Yield }(\%)^{*}\end{array}$ \\
\hline 1 & $\mathbf{A 3}$ & DCM & DIPEA & 20 & 97 & 10 \\
\hline 2 & $\mathbf{A 3}$ & toluene & DIPEA & 17 & 96 & 10 \\
\hline 3 & $\mathbf{A 3}$ & THF & DIPEA & 40 & 94 & 8 \\
\hline 4 & A4 & Dioxane & DIPEA & 28 & 95 & 15 \\
\hline 5 & $\mathbf{A 3}$ & $\mathrm{CH}_{3} \mathrm{CN}$ & DIPEA & trace & n.d. & trace \\
\hline 6 & A1 & THF & DIPEA & trace & n.d. & trace \\
\hline 7 & A2 & THF & DIPEA & trace & n.d. & trace \\
\hline 8 & A4 & THF & DIPEA & 15 & 88 & 12 \\
\hline 9 & $\mathbf{A 3}$ & THF & DBU & trace & n.d. & trace \\
\hline 10 & $\mathbf{A 3}$ & THF & $\mathrm{Cs}_{2} \mathrm{CO}_{3}$ & trace & n.d. & 20 \\
\hline 11 & $\mathbf{A 3}$ & THF & $\mathrm{k}_{2} \mathrm{CO}_{3}$ & 33 & 91 & trace \\
\hline
\end{tabular}

Reactions were performed with $5 \mathbf{a}(0.15 \mathrm{mmol}, 1.0$ equiv. $), 2 \mathbf{c}(0.60 \mathrm{mmol}, 4.0$ equiv. $)$, cat. A (10 mol\%), base ( $0.30 \mathrm{mmol}, 2.0$ equiv.) and $4 \AA$ molecular sevie $(100 \mathrm{mg})$ in solvent $(2.0 \mathrm{~mL})$ at $24{ }^{\circ} \mathrm{C}$ for 36 h. *Isolated yield. †Determined by HPLC analysis on a chiral stationary phase. n.d. = not determined. 
5.2. General procedure for the synthesis of indole-derived bridged biaryls.

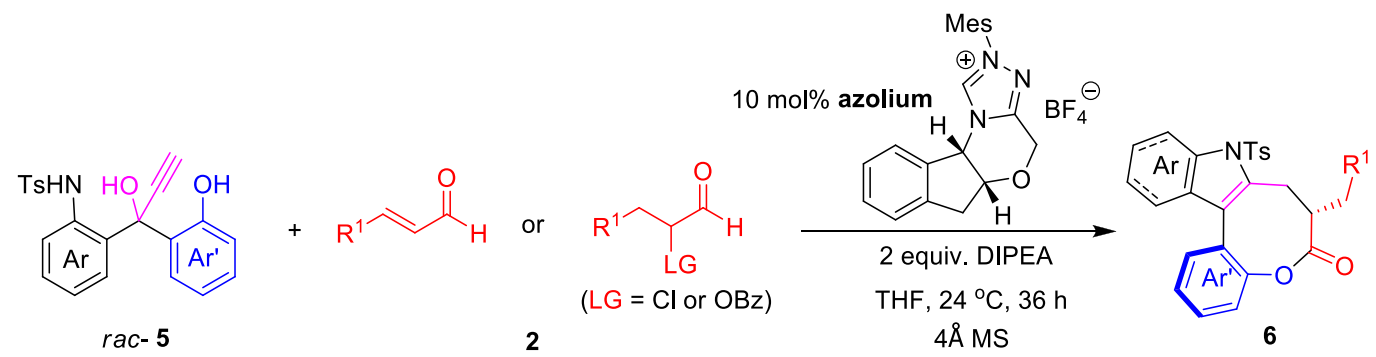

To a $4 \mathrm{~mL}$ vial was added substrate rac $5(0.15 \mathrm{mmol})$ and azolium $(0.015 \mathrm{mmol})$. The mixture was taken into glovebox, where aldehyde $2(0.60 \mathrm{mmol}), 4 \AA$ molecular sieve $(100$ $\mathrm{mg})$, anhydrous THF $(2.0 \mathrm{~mL})$ and $N, N$-diisopropylethylamine $(0.30 \mathrm{mmol})$ were added. The reaction mixture was taken outside the glovebox and the reaction mixture was allowed to stir at $24{ }^{\circ} \mathrm{C}$ for $36 \mathrm{~h}$. The crude reaction mixture was directly purified by silica gel column chromatography with hexanes/ethyl acetate $(20: 1 \mathrm{v} / \mathrm{v})$ as eluent to afford product 6 in a pure form. 


\section{Characterization of indole-derived bridged biaryls.}

(S)-7-butyl-9-tosyl-7,8-dihydrobenzo[2,3] oxocino[5,4-b]indol-6(9H)-one. (Scheme 5, 6a)<smiles>O=C1CC2=C(c3ccccc3[NH2+]2)c2ccccc2O1</smiles>

Used Enal 2c. White solid, MP: $130-132{ }^{\circ} \mathrm{C} .{ }^{1} \mathbf{H}$ NMR (500 MHz, $\mathrm{CDCl}_{3}$ ): $\delta 8.20$ (d, $J=8.5$ $\mathrm{Hz}, 1 \mathrm{H}), 7.70(\mathrm{~d}, J=8.0 \mathrm{~Hz}, 2 \mathrm{H}), 7.48-7.45(\mathrm{~m}, 2 \mathrm{H}), 7.33(\mathrm{~d}, J=8.5 \mathrm{~Hz}, 1 \mathrm{H}), 7.29(\mathrm{~d}, J=$ $8.0 \mathrm{~Hz}, 1 \mathrm{H}), 7.25-7.19(\mathrm{~m}, 5 \mathrm{H}), 3.75(\mathrm{dd}, J=18.514 .0 \mathrm{~Hz}, 1 \mathrm{H}), 3.36-3.30(\mathrm{~m}, 2 \mathrm{H}), 2.38$ (s, $3 \mathrm{H}), 1.93-1.90(\mathrm{~m}, 1 \mathrm{H}), 1.59-1.55(\mathrm{~m}, 1 \mathrm{H}), 1.32-1.22(\mathrm{~m}, 3 \mathrm{H}), 1.19-1.15(\mathrm{~m}, 1 \mathrm{H}), 0.88(\mathrm{t}$, $J=7.0 \mathrm{~Hz}, 3 \mathrm{H}) .{ }^{13} \mathbf{C}$ NMR $\left(125 \mathrm{MHz}, \mathrm{CDCl}_{3}\right): \delta 174.10,149.77,145.12,136.51,135.75$, $134.15,132.53,130.11,129.76,129.75,126.45,126.10,125.99,125.16,123.71,121.17$, $119.35,117.01,114.71,38.31,33.58,33.37,29.60,22.36,21.59,13.80$. HRMS (ESI) m/z Calcd for $\left[\mathrm{C}_{28} \mathrm{H}_{27} \mathrm{NNaO}_{4} \mathrm{~S}, \mathrm{M}+\mathrm{Na}\right]^{+}:$496.1553; Found 496.1556.

Optical Rotation: $[\alpha]^{25}$ - $28.0\left(c=0.1, \mathrm{CHCl}_{3}\right) .94 \%$ ee (HPLC condition: Chiralpak ID column, $n$-Hexane $i-\mathrm{PrOH}=80: 20$, flow rate $=1.0 \mathrm{~mL} / \mathrm{min}$, wave length $=254 \mathrm{~nm}, t_{\mathrm{R}}=$ $11.55 \mathrm{~min}$ for minor isomer, $t_{\mathrm{R}}=15.99 \mathrm{~min}$ for major isomer).

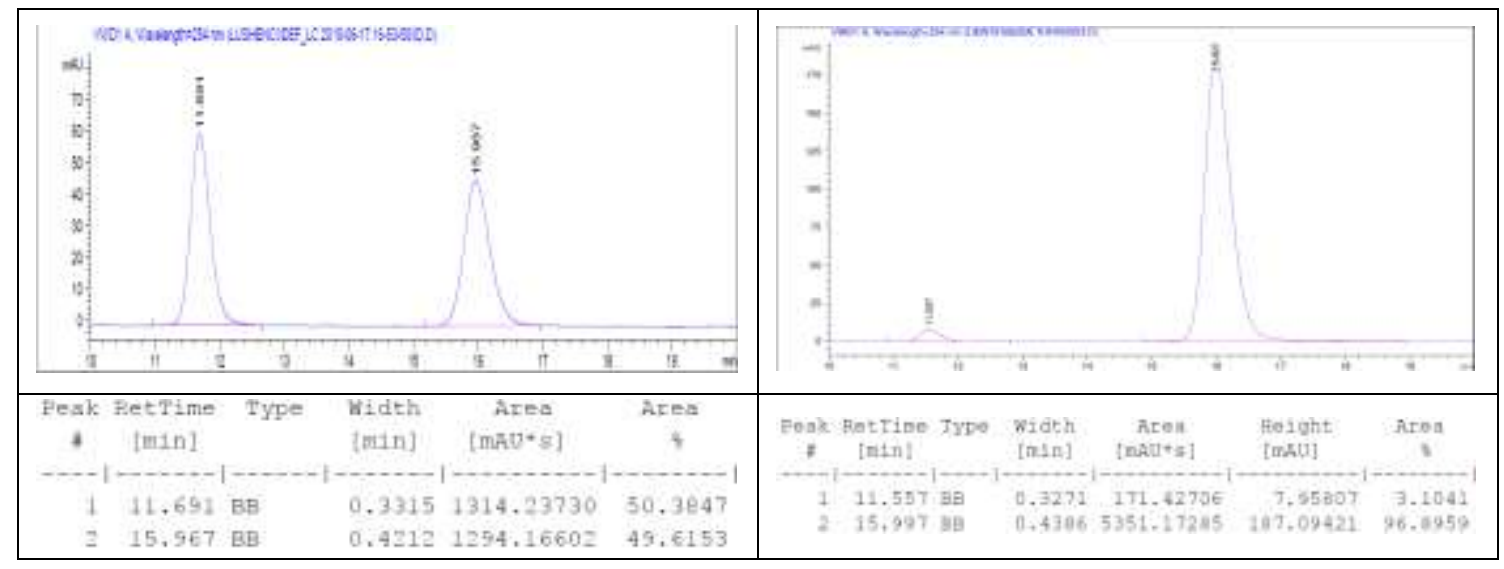

(S)-7-butyl-2-methyl-9-tosyl-7,8-dihydrobenzo[2,3] oxocino[5,4-b]indol-6(9H)-one. (Scheme $5,6 \mathbf{b})$

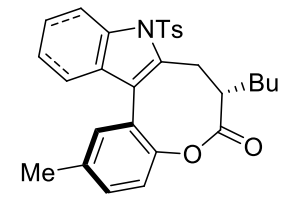

Used Enal 2c. White solid, MP: $137-138{ }^{\circ} \mathrm{C} .{ }^{1} \mathbf{H}$ NMR (500 MHz, $\left.\mathrm{CDCl}_{3}\right): \delta 8.20$ (d, $J=8.5$ $\mathrm{Hz}, 1 \mathrm{H}), 7.69(\mathrm{~d}, J=8.5 \mathrm{~Hz}, 2 \mathrm{H}), 7.33(\mathrm{td}, J=8.51 .0 \mathrm{~Hz}, 1 \mathrm{H}), 7.27-7.20(\mathrm{~m}, 6 \mathrm{H}), 7.12(\mathrm{~d}, J$ $=8.5 \mathrm{~Hz}, 1 \mathrm{H}), 3.74(\mathrm{dd}, J=17.012 .0 \mathrm{~Hz}, 1 \mathrm{H}), 3.39-3.28(\mathrm{~m}, 2 \mathrm{H}), 2.42(\mathrm{~s}, 3 \mathrm{H}), 2.38(\mathrm{~s}, 3 \mathrm{H})$, $1.94-1.88(\mathrm{~m}, 1 \mathrm{H}), 1.57-1.53(\mathrm{~m}, 1 \mathrm{H}), 1.34-1.27(\mathrm{~m}, 3 \mathrm{H}), 1.25-1.14(\mathrm{~m}, 1 \mathrm{H}), 0.89(\mathrm{t}, J=$ $7.5 \mathrm{~Hz}, 3 \mathrm{H}) .{ }^{13} \mathbf{C}$ NMR $\left(125 \mathrm{MHz}, \mathrm{CDCl}_{3}\right): \delta 174.40,147.59,145.09,136.51,135.86,135.75$, $134.08,132.81,130.33,130.14,130.10,126.44,125.53,125.10,123.68,120.86,119.41$, $117.16,114.71,38.20,33.60,33.38,29.63,22.39,21.59,20.84,13.83$. HRMS (ESI) m/z 
Calcd for $\left[\mathrm{C}_{29} \mathrm{H}_{29} \mathrm{NNaO}_{4} \mathrm{~S}, \mathrm{M}+\mathrm{Na}\right]^{+}:$510.1709; Found 510.1706.

Optical Rotation: $[\alpha]^{25}$ - $48.0\left(c=0.3, \mathrm{CHCl}_{3}\right) .97 \%$ ee (HPLC condition: Chiralpak ID column, $n$-Hexane $/ i-\mathrm{PrOH}=80: 20$, flow rate $=1.0 \mathrm{~mL} / \mathrm{min}$, wave length $=254 \mathrm{~nm}, t_{\mathrm{R}}=$ $14.32 \mathrm{~min}$ for minor isomer, $t_{\mathrm{R}}=17.49 \mathrm{~min}$ for major isomer).

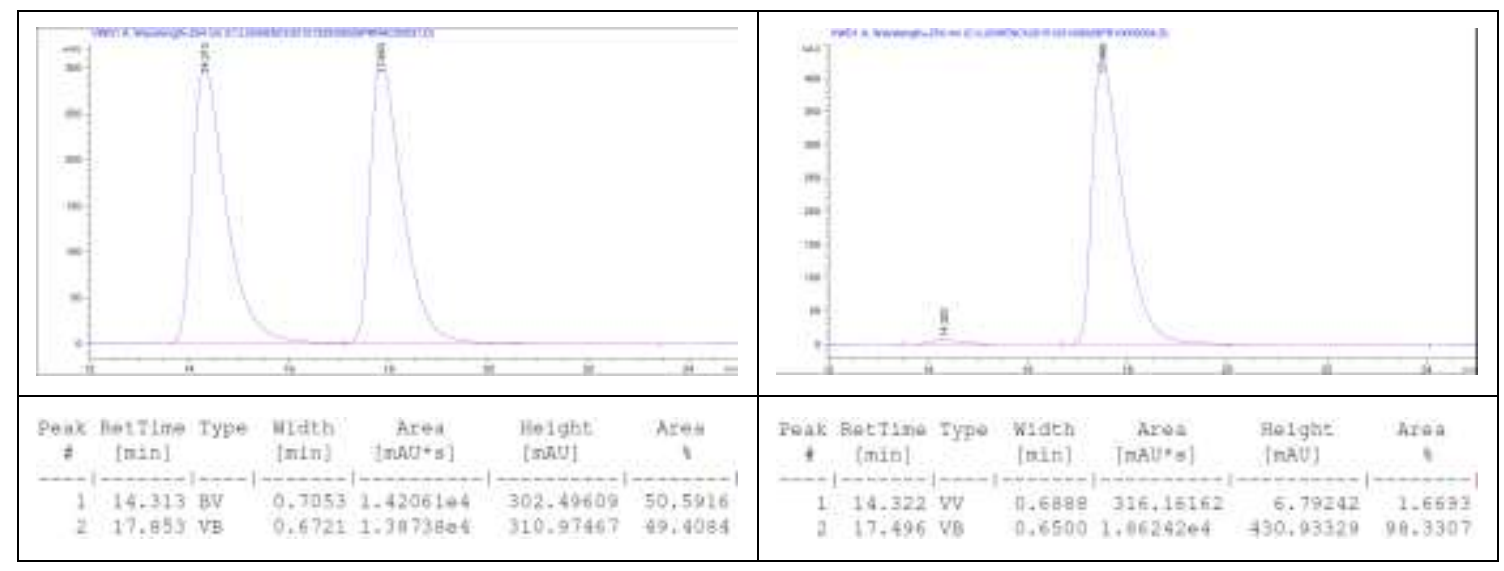

(S)-2-(tert-butyl)-7-butyl-9-tosyl-7,8-dihydrobenzo[2,3] oxocino[5,4-b]indol-6(9H)-one.

(Scheme 5, 6c)

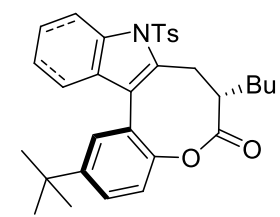

Used Enal 2c. White solid, MP: $91-92{ }^{\circ} \mathrm{C} .{ }^{1} \mathbf{H}$ NMR (500 MHz, $\left.\mathrm{CDCl}_{3}\right): \delta 8.22(\mathrm{~d}, J=8.0$ $\mathrm{Hz}, 1 \mathrm{H}), 7.71(\mathrm{~d}, J=8.5 \mathrm{~Hz}, 2 \mathrm{H}), 7.46-7.44(\mathrm{~m}, 2 \mathrm{H}), 7.35-7.32(\mathrm{~m}, 1 \mathrm{H}), 7.30-7.28(\mathrm{~m}$, 2H), $7.23-7.22(\mathrm{~m}, 2 \mathrm{H}), 7.15(\mathrm{dd}, J=7.51 .5 \mathrm{~Hz}, 1 \mathrm{H}), 3.74(\mathrm{dd}, J=18.513 .5 \mathrm{~Hz}, 1 \mathrm{H}), 3.39$ - $3.32(\mathrm{~m}, 2 \mathrm{H}), 2.38(\mathrm{~s}, 3 \mathrm{H}), 1.93-190(\mathrm{~m}, 1 \mathrm{H}), 1.58-1.55(\mathrm{~m}, 1 \mathrm{H}), 1.38(\mathrm{~s}, 9 \mathrm{H}), 1.35-1.24$ $(\mathrm{m}, 3 \mathrm{H}), 1.21-1.16(\mathrm{~m}, 1 \mathrm{H}), 0.89(\mathrm{t}, J=7.0 \mathrm{~Hz}, 3 \mathrm{H}) .{ }^{13} \mathbf{C} \mathbf{~ N M R}\left(125 \mathrm{MHz}, \mathrm{CDCl}_{3}\right): \delta 174.47$, $149.08,147.36,145.10,136.53,135.81,134.02,130.28,130.13,129.65,126.62,126.49$, $125.05,125.02,123.75,120.49,119.31,117.46,114.73,38.27,34.68,33.64,33.44,31.38$, 29.64, 22.46, 21.60, 13.84. HRMS (ESI) m/z Calcd for $\left[\mathrm{C}_{32} \mathrm{H}_{35} \mathrm{NNaO}_{4} \mathrm{~S}, \mathrm{M}+\mathrm{Na}\right]^{+}:$552.2179; Found 552.2182.

Optical Rotation: $[\alpha]^{25}$ - $90.0\left(c=0.3, \mathrm{CHCl}_{3}\right) .97 \%$ ee (HPLC condition: Chiralpak IA column, $n$-Hexane $/ i-\mathrm{PrOH}=90: 10$, flow rate $=1.0 \mathrm{~mL} / \mathrm{min}$, wave length $=254 \mathrm{~nm}, t_{\mathrm{R}}=$ $5.47 \mathrm{~min}$ for minor isomer, $t_{\mathrm{R}}=7.18 \mathrm{~min}$ for major isomer).

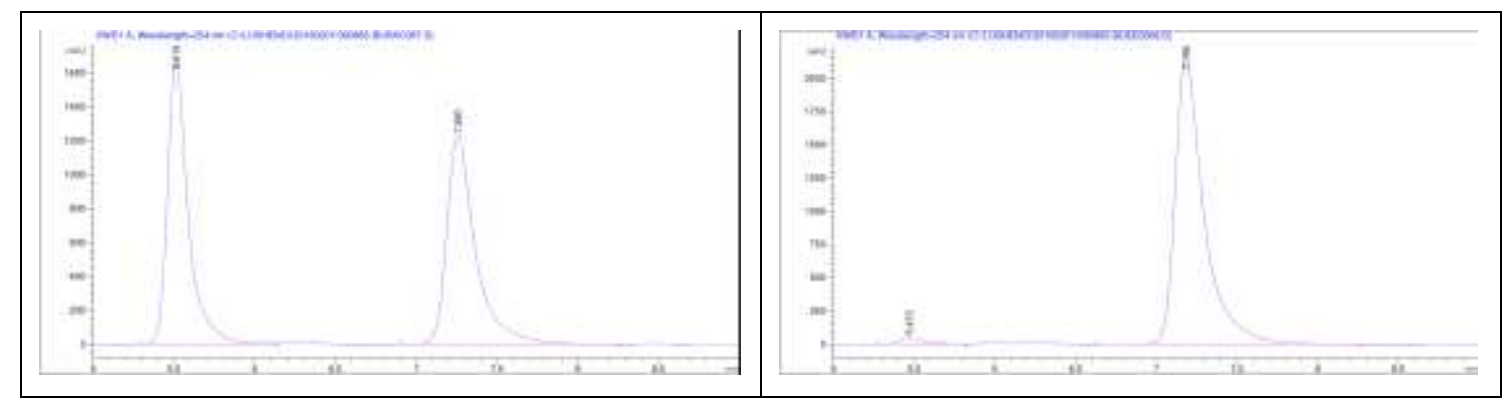




\begin{tabular}{|c|c|c|c|c|c|c|c|c|c|c|c|c|c|}
\hline Prak & $\begin{array}{l}\text { Dettine } \\
\text { [ain] }\end{array}$ & Type & $\begin{array}{l}\text { Wiath } \\
\text { imin] }\end{array}$ & $\begin{array}{c}\text { Axea } \\
\text { [ntorot }\end{array}$ & $\begin{array}{l}\text { felght } \\
\text { (maO) }\end{array}$ & Axen & $\begin{array}{c}\text { Feak } \\
\text {; }\end{array}$ & $\begin{array}{c}\text { EotTine } \\
\text { [nin] }\end{array}$ & TYDo & $\begin{array}{l}\text { Wideh } \\
\text { [min] }\end{array}$ & $\begin{array}{c}\text { Aron } \\
\text { [rad }\end{array}$ & $\begin{array}{l}\text { Berght } \\
\text { [mhou }\end{array}$ & Aron \\
\hline 1 & 5,515 & EV & 0.1340 & 1.5015006 & 1669.99890 & 49.7054 & 1 & 5.473 & ED & 0.1327 & $438+63492$ & 49.39607 & 1.6135 \\
\hline 2 & 7,260 & DV & 0.1853 & 1.5192406 & 1226.56775 & 50.2936 & 2 & 3.176 & 파 & 0.1870 & 2,6714504 & $2134+73120$ & 98.3565 \\
\hline
\end{tabular}

(S)-7-butyl-3-methyl-9-tosyl-7,8-dihydrobenzo[2,3]oxocino[5,4-b]indol-6(9H)-one. (Scheme $5,6 \mathbf{d})$<smiles></smiles>

Used Enal 2c. White solid, MP: $134-135{ }^{\circ} \mathrm{C} .{ }^{1} \mathbf{H}$ NMR (500 MHz, $\left.\mathrm{CDCl}_{3}\right): \delta 8.20$ (d, $J=8.5$ $\mathrm{Hz}, 1 \mathrm{H}), 7.69(\mathrm{~d}, J=8.5 \mathrm{~Hz}, 2 \mathrm{H}), 7.34-7.24(\mathrm{~m}, 5 \mathrm{H}), 7.21-7.18(\mathrm{~m}, 2 \mathrm{H}), 7.05(\mathrm{~s}, 1 \mathrm{H}), 3.73$ $(\mathrm{dd}, J=17.012 .0 \mathrm{~Hz}, 1 \mathrm{H}), 3.37-3.29(\mathrm{~m}, 2 \mathrm{H}), 2.46(\mathrm{~s}, 3 \mathrm{H}), 2.38(\mathrm{~s}, 3 \mathrm{H}), 1.93-1.89(\mathrm{~m}, 1 \mathrm{H})$, $1.59-1.53(\mathrm{~m}, 1 \mathrm{H}), 1.34-1.20(\mathrm{~m}, 3 \mathrm{H}), 1.20-1.14(\mathrm{~m}, 1 \mathrm{H}), 0.90(\mathrm{t}, J=7.0 \mathrm{~Hz}, 3 \mathrm{H}) .{ }^{13} \mathrm{C}$ NMR $\left(125 \mathrm{MHz}, \mathrm{CDCl}_{3}\right): \delta 174.30,149.54,145.08,140.32,136.49,135.75,133.99,132.19$, $130.25,130.10,126.93,126.45,125.08,123.65,122.75,121.55,119.42,117.07,114.68$, 38.38, 33.57, 33.45, 29.61, 22.45, 21.60, 21.13, 13.83. HRMS (ESI) $\mathrm{m} / \mathrm{z}$ Calcd for $\left[\mathrm{C}_{29} \mathrm{H}_{29} \mathrm{NNaO}_{4} \mathrm{~S}, \mathrm{M}+\mathrm{Na}\right]^{+}:$510.1709; Found 510.1705.

Optical Rotation: $[\alpha]^{25}$ - $65.0\left(c=0.2, \mathrm{CHCl}_{3}\right) .95 \%$ ee (HPLC condition: Chiralpak IA column, $n$-Hexane $/ i-\mathrm{PrOH}=90: 10$, flow rate $=1.0 \mathrm{~mL} / \mathrm{min}$, wave length $=254 \mathrm{~nm}, t_{\mathrm{R}}=$ $7.37 \mathrm{~min}$ for minor isomer, $t_{\mathrm{R}}=14.42 \mathrm{~min}$ for major isomer).

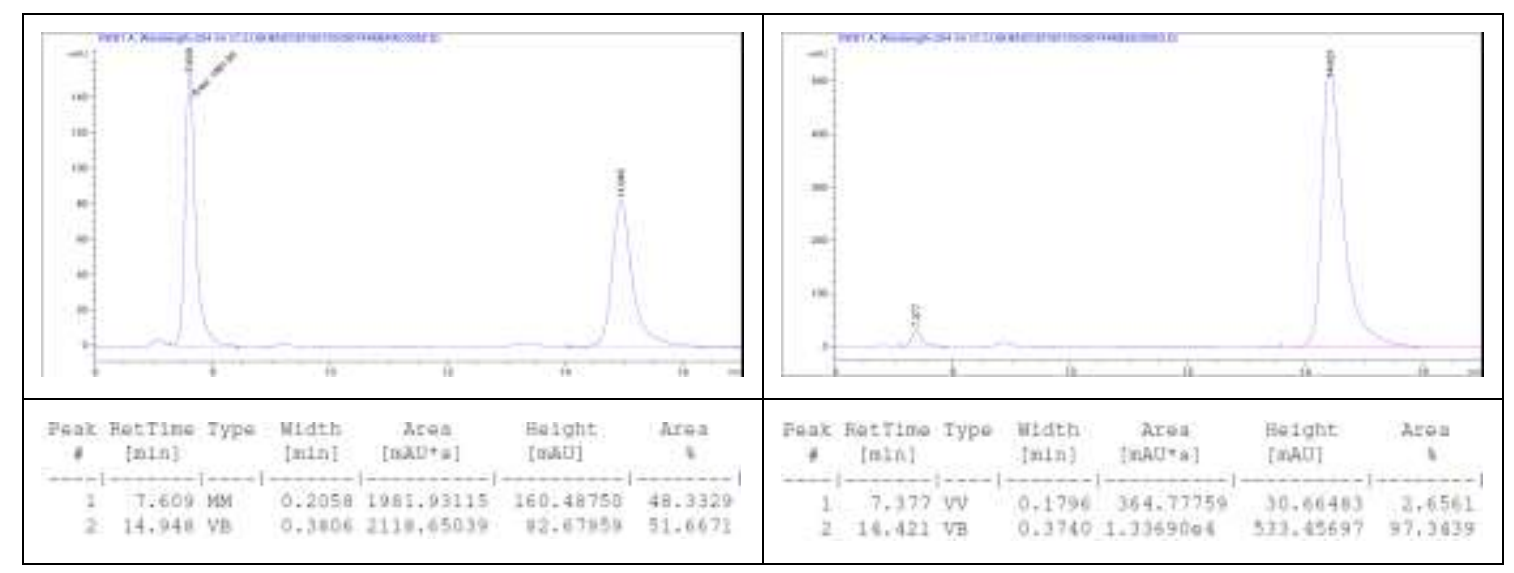

(S)-7-butyl-2,12-dimethyl-9-tosyl-7,8-dihydrobenzo[2,3] oxocino[5,4-b]indol-6(9H)-one.

(Scheme 5, 6e)

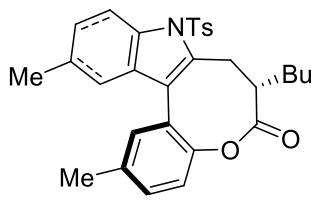

Used Enal 2c. White solid, MP: $84-85^{\circ} \mathrm{C} .{ }^{1} \mathbf{H}$ NMR (500 MHz, $\left.\mathrm{CDCl}_{3}\right): \delta 8.07(\mathrm{~d}, J=8.5$ 
$\mathrm{Hz}, 1 \mathrm{H}), 7.67(\mathrm{~d}, J=8.5 \mathrm{~Hz}, 2 \mathrm{H}), 7.27-7.23(\mathrm{~m}, 4 \mathrm{H}), 7.14(\mathrm{dd}, J=9.01 .0 \mathrm{~Hz}, 1 \mathrm{H}), 7.11(\mathrm{~d}, J$ $=8.0 \mathrm{~Hz}, 1 \mathrm{H}), 7.02(\mathrm{~s}, 1 \mathrm{H}), 3.72(\mathrm{dd}, J=17.012 .0 \mathrm{~Hz}, 1 \mathrm{H}), 3.36-3.25(\mathrm{~m}, 2 \mathrm{H}), 2.44(\mathrm{~s}, 3 \mathrm{H})$, $2.37(\mathrm{~s}, 6 \mathrm{H}), 1.94-1.88(\mathrm{~m}, 1 \mathrm{H}), 1.58-1.53(\mathrm{~m}, 1 \mathrm{H}), 1.31-1.24(\mathrm{~m}, 3 \mathrm{H}), 1.20-1.15(\mathrm{~m}$, $1 \mathrm{H}), 0.88(\mathrm{t}, J=7.5 \mathrm{~Hz}, 3 \mathrm{H}) .{ }^{13} \mathrm{C}$ NMR $\left(125 \mathrm{MHz}, \mathrm{CDCl}_{3}\right): \delta 174.39,147.60,144.96,135.83$, $135.76,134.75,134.11,133.38,132.81,130.33,130.28,130.05,126.48,126.42,125.67$, 120.84, 119.18, 117.03, 114.43, 38.23, 33.56, 33.37, 29.63, 22.39, 21.58, 21.23, 20.88, 13.83 .

HRMS (ESI) m/z Calcd for $\left[\mathrm{C}_{30} \mathrm{H}_{31} \mathrm{NNaO}_{4} \mathrm{~S}, \mathrm{M}+\mathrm{Na}\right]^{+}:$524.1866; Found 524.1869.

Optical Rotation: $[\alpha]^{25}$ - $48.0\left(c=0.3, \mathrm{CHCl}_{3}\right) .97 \%$ ee (HPLC condition: Chiralpak IA column, $n$-Hexane $/ i-\mathrm{PrOH}=90: 10$, flow rate $=1.0 \mathrm{~mL} / \mathrm{min}$, wave length $=254 \mathrm{~nm}, t_{\mathrm{R}}=$ $5.20 \mathrm{~min}$ for minor isomer, $t_{\mathrm{R}}=6.18 \mathrm{~min}$ for major isomer).

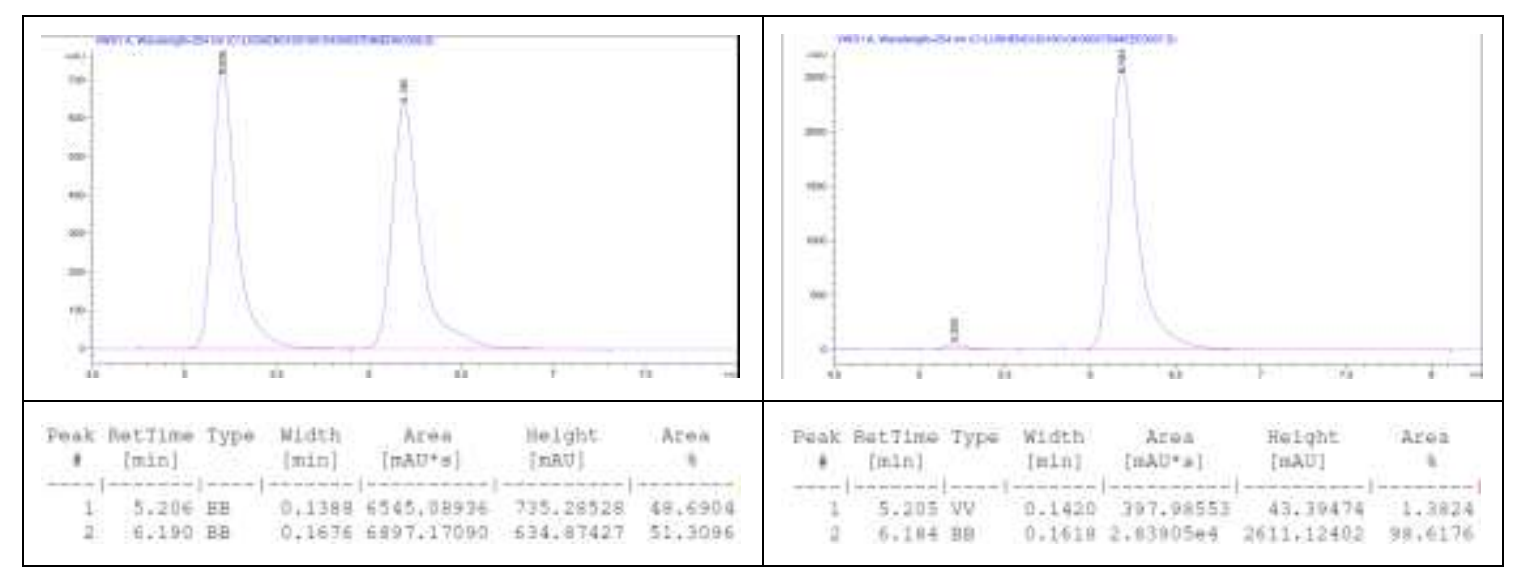

(S)-12-bromo-7-butyl-2-methyl-9-tosyl-7,8-dihydrobenzo[2,3] oxocino[5,4-b]indol-6(9H)-one.

(Scheme 5, 6f)

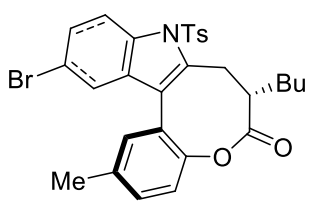

Used Enal 2c. White solid, MP: $138-139{ }^{\circ} \mathrm{C} .{ }^{1} \mathbf{H}$ NMR (500 MHz, $\left.\mathrm{CDCl}_{3}\right): \delta 8.08(\mathrm{~d}, J=9.0$ $\mathrm{Hz}, 1 \mathrm{H}), 7.67$ (d, $J=7.5 \mathrm{~Hz}, 2 \mathrm{H}), 7.43$ (dd, $J=9.02 .0 \mathrm{~Hz}, 1 \mathrm{H}), 7.37$ (d, $J=2.0 \mathrm{~Hz}, 1 \mathrm{H}), 7.30$ - $7.25(\mathrm{~m}, 3 \mathrm{H}), 7.19(\mathrm{~d}, J=2.0 \mathrm{~Hz}, 1 \mathrm{H}), 7.12(\mathrm{~d}, J=8.5 \mathrm{~Hz}, 1 \mathrm{H}), 3.74-3.67(\mathrm{~m}, 1 \mathrm{H}), 3.34$ $3.26(\mathrm{~m}, 2 \mathrm{H}), 2.44(\mathrm{~s}, 3 \mathrm{H}), 2.39(\mathrm{~s}, 3 \mathrm{H}), 1.92-1.88(\mathrm{~m}, 1 \mathrm{H}), 1.57-1.53(\mathrm{~m}, 1 \mathrm{H}), 1.33-1.23$ $(\mathrm{m}, 3 \mathrm{H}), 1.18-1.14(\mathrm{~m}, 1 \mathrm{H}), 0.88(\mathrm{t}, J=7.5 \mathrm{~Hz}, 3 \mathrm{H}) .{ }^{13} \mathbf{C} \mathbf{N M R}\left(125 \mathrm{MHz}, \mathrm{CDCl}_{3}\right): \delta 174.19$, $147.56,145.47,136.21,135.43,135.41,135.22,132.54,131.86,130.71,130.24,127.98$, $126.44,124.89,122.01,120.99,117.31,116.43,116.18,38.15,33.50,33.33,29.60,22.37$, 21.63, 20.91, 13.82. HRMS (ESI) $\mathrm{m} / \mathrm{z}$ Calcd for $\left[\mathrm{C}_{29} \mathrm{H}_{28}{ }^{79} \mathrm{BrNNaO}_{4} \mathrm{~S}, \mathrm{M}+\mathrm{Na}\right]^{+}:$588.0814; Found 588.0812; $\left[\mathrm{C}_{29} \mathrm{H}_{28}{ }^{81} \mathrm{BrNNaO}_{4} \mathrm{~S}, \mathrm{M}+\mathrm{Na}\right]^{+}$: 590.0794; Found 590.0797.

Optical Rotation: $[\alpha]^{25}$ D $92.0\left(c=0.1, \mathrm{CHCl}_{3}\right) .94 \%$ ee (HPLC condition: Chiralpak IA column, $n$-Hexane $/ i-\mathrm{PrOH}=90: 10$, flow rate $=1.0 \mathrm{~mL} / \mathrm{min}$, wave length $=254 \mathrm{~nm}, t_{\mathrm{R}}=$ $6.57 \mathrm{~min}$ for minor isomer, $t_{\mathrm{R}}=7.99 \mathrm{~min}$ for major isomer). 


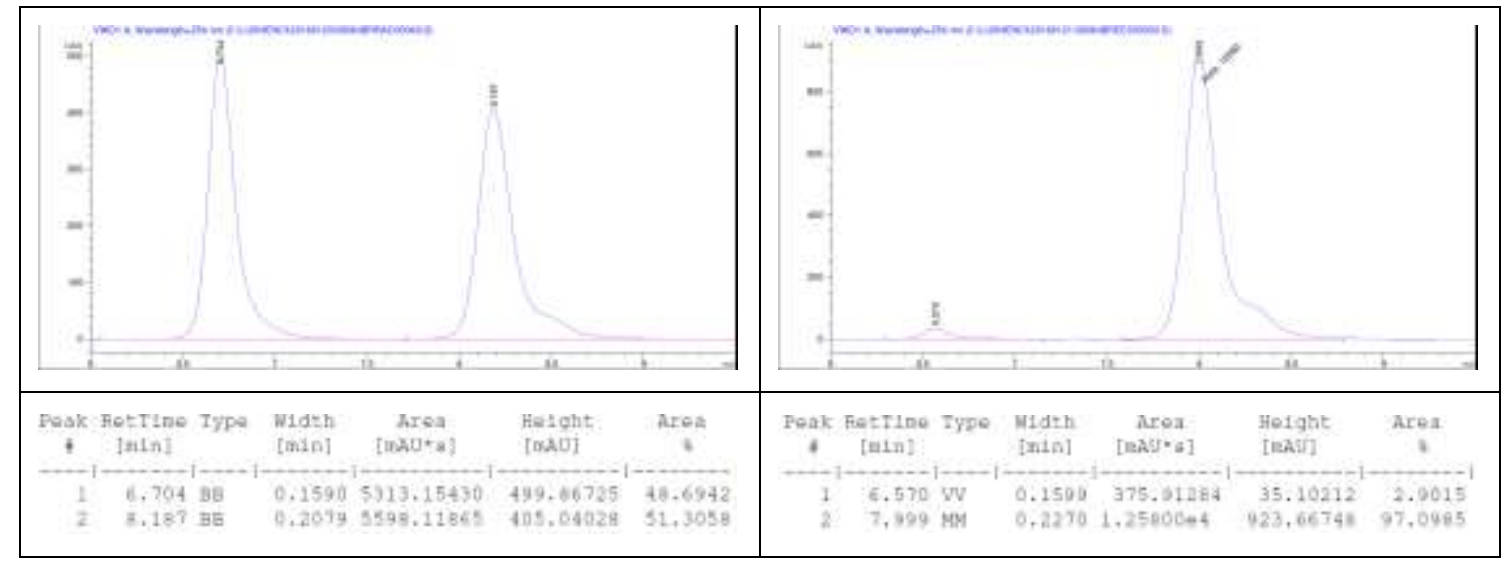

(S)-7-propyl-9-tosyl-7,8-dihydrobenzo[2,3] oxocino[5,4-b]indol-6(9H)-one. (Scheme 4, 6g)<smiles></smiles>

Used Enal 2b. White solid, MP: $102-104{ }^{\circ} \mathrm{C} . .{ }^{1} \mathbf{H}$ NMR $\left(500 \mathrm{MHz}, \mathrm{CDCl}_{3}\right): \delta 8.21(\mathrm{~d}, J=$ $8.5 \mathrm{~Hz}, 1 \mathrm{H}), 7.70(\mathrm{~d}, J=8.5 \mathrm{~Hz}, 2 \mathrm{H}), 7.47-7.44(\mathrm{~m}, 2 \mathrm{H}), 7.39(\mathrm{dd}, J=7.51 .0 \mathrm{~Hz}, 1 \mathrm{H}), 7.36-$ $7.19(\mathrm{~m}, 6 \mathrm{H}), 3.75(\mathrm{dd}, J=17.012 .0 \mathrm{~Hz}, 1 \mathrm{H}), 3.38-3.29(\mathrm{~m}, 2 \mathrm{H}), 2.38(\mathrm{~s}, 3 \mathrm{H}), 1.95-1.92$ $(\mathrm{m}, 1 \mathrm{H}), 1.56-1.51(\mathrm{~m}, 1 \mathrm{H}), 1.39-1.37(\mathrm{~m}, 1 \mathrm{H}), 1.28-1.22(\mathrm{~m}, 1 \mathrm{H}), 0.85(\mathrm{t}, J=7.5 \mathrm{~Hz}, 3 \mathrm{H})$. ${ }^{13} \mathrm{C}$ NMR $\left(125 \mathrm{MHz}, \mathrm{CDCl}_{3}\right): \delta 174.09,149.75,145.14,136.51,135.74,134.14,132.54$, $130.13,130.12,129.76,126.45,126.12,125.99,125.17,123.72,121.18,119.36,117.01$, 114.71, 37.99, 35.64, 33.58, 21.60, 20.65, 13.64. HRMS (ESI) $\mathrm{m} / \mathrm{z}$ Calcd for $\left[\mathrm{C}_{27} \mathrm{H}_{25} \mathrm{NNaO}_{4} \mathrm{~S}, \mathrm{M}+\mathrm{Na}\right]^{+}:$482.1396; Found 482.1398 .

Optical Rotation: $[\alpha]^{25}$ - $68.0\left(c=0.1, \mathrm{CHCl}_{3}\right) .92 \%$ ee (HPLC condition: Chiralpak ID column, $n$-Hexane $/ i-\mathrm{PrOH}=80: 20$, flow rate $=1.0 \mathrm{~mL} / \mathrm{min}$, wave length $=254 \mathrm{~nm}, t_{\mathrm{R}}=$ $12.00 \mathrm{~min}$ for minor isomer, $t_{\mathrm{R}}=16.24 \mathrm{~min}$ for major isomer).

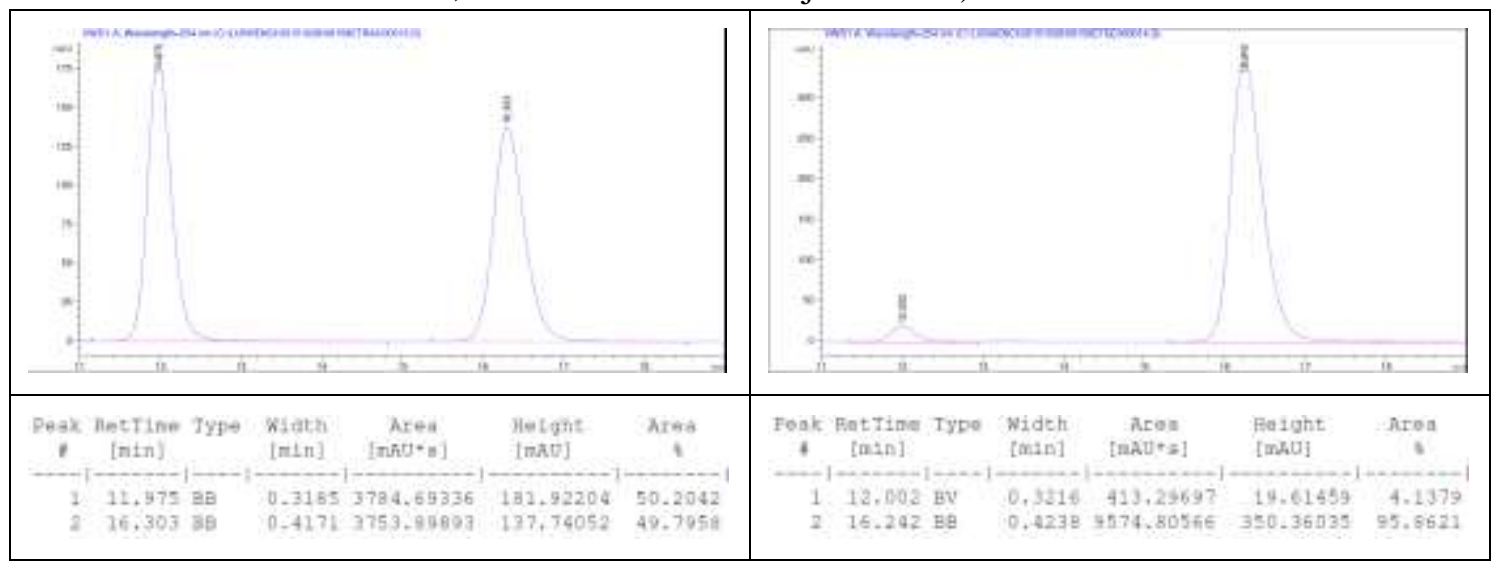

(S)-2,11-dimethyl-7-propyl-9-tosyl-7,8-dihydrobenzo[2,3]oxocino[5,4-b]indol-6(9H)-one. (Scheme 5, 6h) 


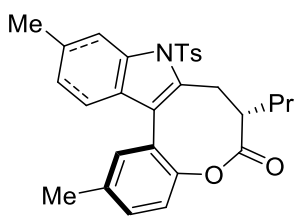

Used Enal 2b. White solid, MP: $193-194{ }^{\circ} \mathrm{C} .{ }^{1} \mathbf{H}$ NMR $\left(500 \mathrm{MHz}, \mathrm{CDCl}_{3}\right): \delta 8.02(\mathrm{~s}, 1 \mathrm{H})$, $7.67(\mathrm{~d}, J=8.5 \mathrm{~Hz}, 2 \mathrm{H}), 7.29-7.27(\mathrm{~m}, 2 \mathrm{H}), 7.23-7.22(\mathrm{~m}, 2 \mathrm{H}), 7.14(\mathrm{~d}, J=8.0 \mathrm{~Hz}, 1 \mathrm{H})$, $7.10(\mathrm{~d}, J=9.0 \mathrm{~Hz}, 1 \mathrm{H}), 7.05(\mathrm{~d}, J=8.0 \mathrm{~Hz}, 1 \mathrm{H}), 3.69(\mathrm{dd}, J=17.512 .5 \mathrm{~Hz}, 1 \mathrm{H}), 3.38-3.33$ (m, 1H), $3.26(\mathrm{dd}, J=17.54 .5 \mathrm{~Hz}, 1 \mathrm{H}), 2.50(\mathrm{~s}, 3 \mathrm{H}), 2.41(\mathrm{~s}, 3 \mathrm{H}), 2.38(\mathrm{~s}, 3 \mathrm{H}), 1.94-1.88(\mathrm{~m}$, $1 \mathrm{H}), 1.53-1.46(\mathrm{~m}, 1 \mathrm{H}), 1.39-1.34(\mathrm{~m}, 1 \mathrm{H}), 1.25-1.19(\mathrm{~m}, 1 \mathrm{H}), 0.84(\mathrm{t}, J=7.5 \mathrm{~Hz}, 3 \mathrm{H})$. ${ }^{13}$ C NMR (125 MHz, $\left.\mathrm{CDCl}_{3}\right): \delta 174.43,147.52,145.00,136.92,135.88,135.82,135.26$, $133.28,132.79,130.22,130.10,127.92,126.37,125.68,125.15,120.83,119.01,117.09$, 114.81, 37.83, 35.62, 33.65, 22.01, 21,61, 20.84, 20.65, 13.67. HRMS (ESI) m/z Calcd for $\left[\mathrm{C}_{29} \mathrm{H}_{29} \mathrm{NNaO}_{4} \mathrm{~S}, \mathrm{M}+\mathrm{Na}\right]^{+}$: 510.1709; Found 510.1713.

Optical Rotation: $[\alpha]^{25} \mathrm{D}-40.0\left(c=0.2, \mathrm{CHCl}_{3}\right) .98 \%$ ee (HPLC condition: Chiralpak IA column, $n$-Hexane $/ i-\mathrm{PrOH}=90: 10$, flow rate $=1.0 \mathrm{~mL} / \mathrm{min}$, wave length $=254 \mathrm{~nm}, t_{\mathrm{R}}=$ $6.51 \mathrm{~min}$ for minor isomer, $t_{\mathrm{R}}=7.70 \mathrm{~min}$ for major isomer).

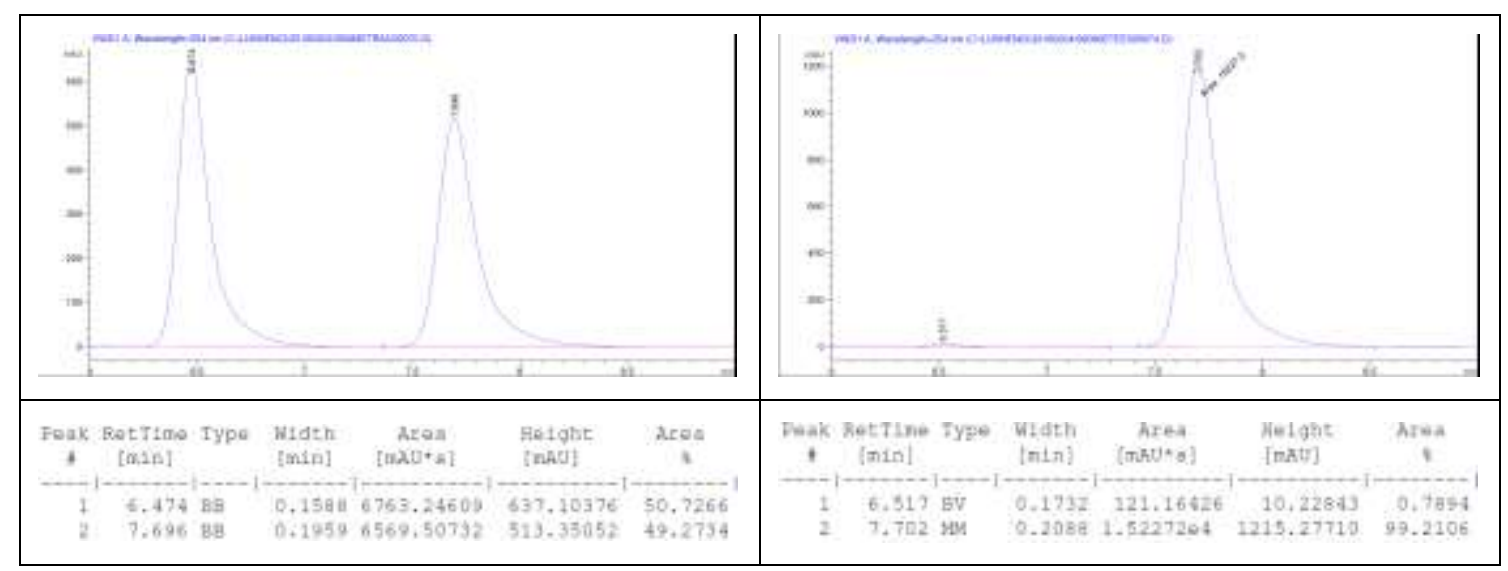

(S)-7-butyl-2,11-dimethyl-9-tosyl-7,8-dihydrobenzo[2,3] oxocino[5,4-b]indol-6(9H)-one.

(Scheme 5, 6i)

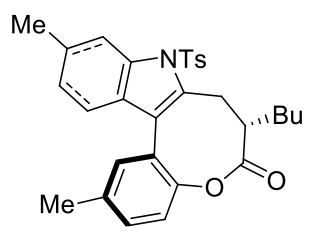

Used Enal 2c. White solid, MP: $135-136{ }^{\circ} \mathrm{C} .{ }^{1} \mathbf{H}$ NMR $\left(500 \mathrm{MHz}, \mathrm{CDCl}_{3}\right): \delta 8.02(\mathrm{~s}, 1 \mathrm{H})$, $7.68(\mathrm{~d}, J=8.5 \mathrm{~Hz}, 2 \mathrm{H}), 7.29-7.27(\mathrm{~m}, 2 \mathrm{H}), 7.24-7.23(\mathrm{~m}, 2 \mathrm{H}), 7.15(\mathrm{~d}, J=8.0 \mathrm{~Hz}, 1 \mathrm{H})$, $7.10(\mathrm{~d}, J=8.5 \mathrm{~Hz}, 1 \mathrm{H}), 7.05(\mathrm{~d}, J=8.0 \mathrm{~Hz}, 1 \mathrm{H}), 3.69(\mathrm{dd}, J=17.012 .0 \mathrm{~Hz}, 1 \mathrm{H}), 3.37-3.26$ (m, 2H), $2.50(\mathrm{~s}, 3 \mathrm{H}), 2.42(\mathrm{~s}, 3 \mathrm{H}), 2.38(\mathrm{~s}, 3 \mathrm{H}), 1.92-1.87(\mathrm{~m}, 1 \mathrm{H}), 1.57-1.50(\mathrm{~m}, 1 \mathrm{H}), 1.31$ - $1.22(\mathrm{~m}, 3 \mathrm{H}), 1.20-1.15(\mathrm{~m}, 1 \mathrm{H}), 0.88(\mathrm{t}, J=7.5 \mathrm{~Hz}, 3 \mathrm{H}) .{ }^{13} \mathbf{C} \mathbf{N M R}\left(125 \mathrm{MHz}, \mathrm{CDCl}_{3}\right): \delta$ $174.43,147.55,144.98,136.93,135.91,135.80,135.25,133.31,132.79,130.23,130.09$, $127.93,126.37,125.68,125.15,120.82,119.01,117.10,114.82,38.18,33.66,33.37,29.63$, 
22.39, 22.00, 21.59, 20.84, 13.83. HRMS (ESI) $\mathrm{m} / \mathrm{z}$ Calcd for $\left[\mathrm{C}_{30} \mathrm{H}_{31} \mathrm{NNaO}_{4} \mathrm{~S}, \mathrm{M}+\mathrm{Na}\right]^{+}$: 524.1866; Found 524.1865.

Optical Rotation: $[\alpha]^{25}$ - $98.0\left(c=0.3, \mathrm{CHCl}_{3}\right) .98 \%$ ee (HPLC condition: Chiralpak IA column, $n$-Hexane $/ i-\mathrm{PrOH}=90: 10$, flow rate $=1.0 \mathrm{~mL} / \mathrm{min}$, wave length $=254 \mathrm{~nm}, t_{\mathrm{R}}=$ $6.22 \mathrm{~min}$ for minor isomer, $t_{\mathrm{R}}=7.67 \mathrm{~min}$ for major isomer).

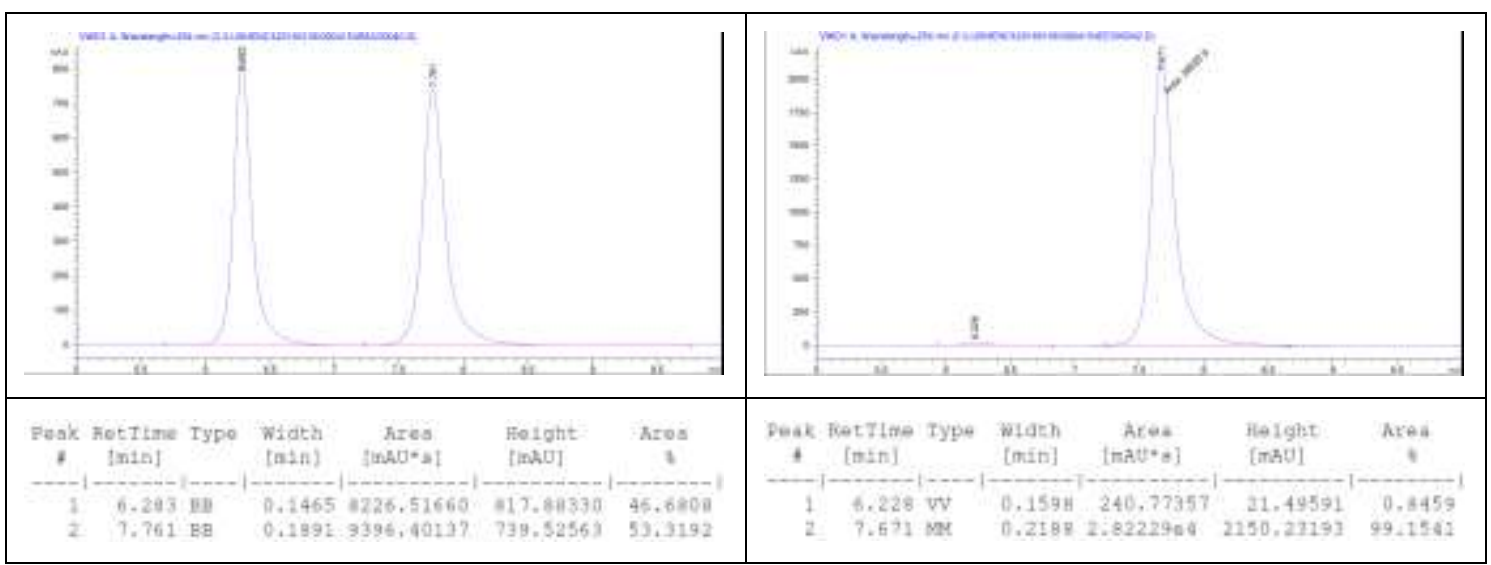

(S)-2,11-dimethyl-7-pentyl-9-tosyl-7,8-dihydrobenzo[2,3] oxocino[5,4-b]indol-6(9H)-one.

(Scheme 5, 6j)

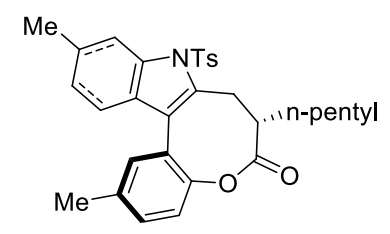

Used Enal 2d. Syrup. ${ }^{1} \mathbf{H}$ NMR (500 MHz, $\left.\mathrm{CDCl}_{3}\right): \delta 8.01(\mathrm{~s}, 1 \mathrm{H}), 7.67(\mathrm{~d}, J=8.5 \mathrm{~Hz}, 2 \mathrm{H})$, $7.29-7.27(\mathrm{~m}, 2 \mathrm{H}), 7.23-7.22(\mathrm{~m}, 2 \mathrm{H}), 7.14(\mathrm{~d}, J=8.0 \mathrm{~Hz}, 1 \mathrm{H}), 7.10(\mathrm{~d}, J=9.0 \mathrm{~Hz}, 1 \mathrm{H})$, $7.05(\mathrm{~d}, J=8.0 \mathrm{~Hz}, 1 \mathrm{H}), 3.68(\mathrm{dd}, J=17.512 .5 \mathrm{~Hz}, 1 \mathrm{H}), 3.37-3.31(\mathrm{~m}, 1 \mathrm{H}), 3.27(\mathrm{dd}, J=$ $17.54 .5 \mathrm{~Hz}, 1 \mathrm{H}), 2.50(\mathrm{~s}, 3 \mathrm{H}), 2.42(\mathrm{~s}, 3 \mathrm{H}), 2.38(\mathrm{~s}, 3 \mathrm{H}), 1.93-1.87(\mathrm{~m}, 1 \mathrm{H}), 1.55-1.50(\mathrm{~m}$, 1H), $1.34-1.21(\mathrm{~m}, 3 \mathrm{H}), 1.19-1.16(\mathrm{~m}, 3 \mathrm{H}), 0.87(\mathrm{t}, J=7.5 \mathrm{~Hz}, 3 \mathrm{H}) .{ }^{13} \mathbf{C}$ NMR (125 MHz, $\left.\mathrm{CDCl}_{3}\right): \delta 174.44,147.53,144.99,136.91,135.88,135.81,135.25,133.30,132.79,130.20$, $130.09,127.92,126.37,125.69,125.14,120.85,119.01,117.08,114.81,38.14,33.66,33.58$, 31.42, 27.11, 22.40, 22.00, 21.60, 20.84, 13.96. HRMS (ESI) $\mathrm{m} / \mathrm{z}$ Calcd for $\left[\mathrm{C}_{31} \mathrm{H}_{33} \mathrm{NNaO}_{4} \mathrm{~S}\right.$, $\mathrm{M}+\mathrm{Na}]^{+}:$538.2022; Found 538.2024.

Optical Rotation: $[\alpha]^{25}$ - $41.0\left(c=0.2, \mathrm{CHCl}_{3}\right) .99 \%$ ee (HPLC condition: Chiralpak IA column, $n$-Hexane $/ i-\mathrm{PrOH}=90: 10$, flow rate $=1.0 \mathrm{~mL} / \mathrm{min}$, wave length $=254 \mathrm{~nm}, t_{\mathrm{R}}=$ $6.05 \mathrm{~min}$ for minor isomer, $t_{\mathrm{R}}=7.39 \mathrm{~min}$ for major isomer). 


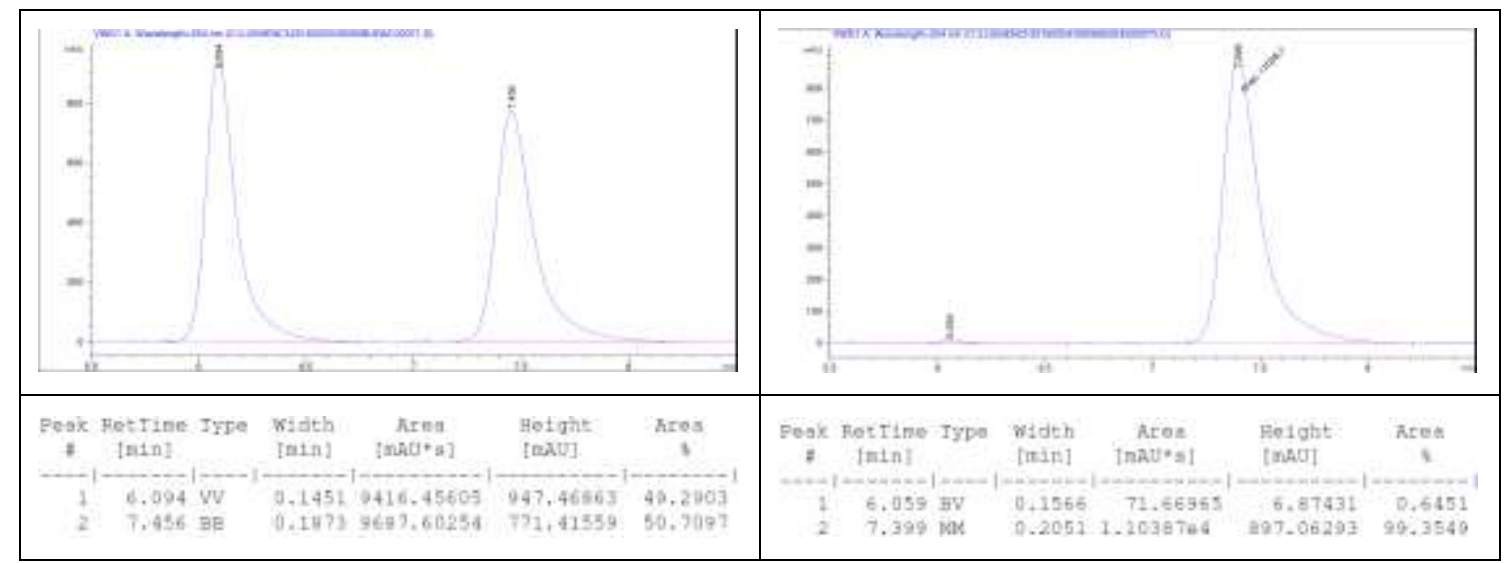

(S)-7-hexyl-2,11-dimethyl-9-tosyl-7,8-dihydrobenzo[2,3] oxocino[5,4-b]indol-6(9H)-one.

(Scheme 5, 6k)<smiles>Cc1ccc2c(c1)C1=C(C[C@H](C)C(=O)Oc3ccc(C)cc31)[NH2+]2</smiles>

Used 2-Chloroaldehyde 2e. Syrup. ${ }^{1} \mathbf{H}$ NMR $\left(500 \mathrm{MHz}, \mathrm{CDCl}_{3}\right): \delta 8.01(\mathrm{~s}, 1 \mathrm{H}), 7.69(\mathrm{~d}, J=$ $8.5 \mathrm{~Hz}, 2 \mathrm{H}), 7.29-7.27(\mathrm{~m}, 2 \mathrm{H}), 7.23-7.22(\mathrm{~m}, 2 \mathrm{H}), 7.14(\mathrm{~d}, J=8.0 \mathrm{~Hz}, 1 \mathrm{H}), 7.10(\mathrm{~d}, J=8.0$ $\mathrm{Hz}, 1 \mathrm{H}), 7.05(\mathrm{~d}, J=8.0 \mathrm{~Hz}, 1 \mathrm{H}), 3.68(\mathrm{dd}, J=17.512 .5 \mathrm{~Hz}, 1 \mathrm{H}), 3.37-3.31(\mathrm{~m}, 1 \mathrm{H}), 3.27$ (dd, $J=17.55 .0 \mathrm{~Hz}, 1 \mathrm{H}), 2.49$ (s, 3H), $2.42(\mathrm{~s}, 3 \mathrm{H}), 2.38(\mathrm{~s}, 3 \mathrm{H}), 1.93-1.87(\mathrm{~m}, 1 \mathrm{H}), 1.55-$ $1.50(\mathrm{~m}, 1 \mathrm{H}), 1.33-1.16(\mathrm{~m}, 8 \mathrm{H}), 0.88(\mathrm{t}, J=7.0 \mathrm{~Hz}, 3 \mathrm{H}) .{ }^{13} \mathbf{C}$ NMR $\left(125 \mathrm{MHz}, \mathrm{CDCl}_{3}\right): \delta$ $174.41,147.54,144.98,136.92,135.90,135.80,135.25,133.31,132.78,130.19,130.08$, $127.92,126.37,125.69,125.14,120.85,119.00,117.08,114.82,38.11,33.67,33.62,31.55$, 28.88, 27.40, 22.51, 22.00, 21.60, 20.83, 14.00. HRMS (ESI) $\mathrm{m} / \mathrm{z}$ Calcd for $\left[\mathrm{C}_{32} \mathrm{H}_{35} \mathrm{NNaO}_{4} \mathrm{~S}\right.$, $\mathrm{M}+\mathrm{Na}]^{+}:$552.2179; Found 552.2175.

Optical Rotation: $[\alpha]^{25}$ - $42.0\left(c=0.1, \mathrm{CHCl}_{3}\right) .99 \%$ ee (HPLC condition: Chiralpak IA column, $n$-Hexane $/ i-\mathrm{PrOH}=90: 10$, flow rate $=1.0 \mathrm{~mL} / \mathrm{min}$, wave length $=254 \mathrm{~nm}, t_{\mathrm{R}}=$ $5.91 \mathrm{~min}$ for minor isomer, $t_{\mathrm{R}}=7.10 \mathrm{~min}$ for major isomer).

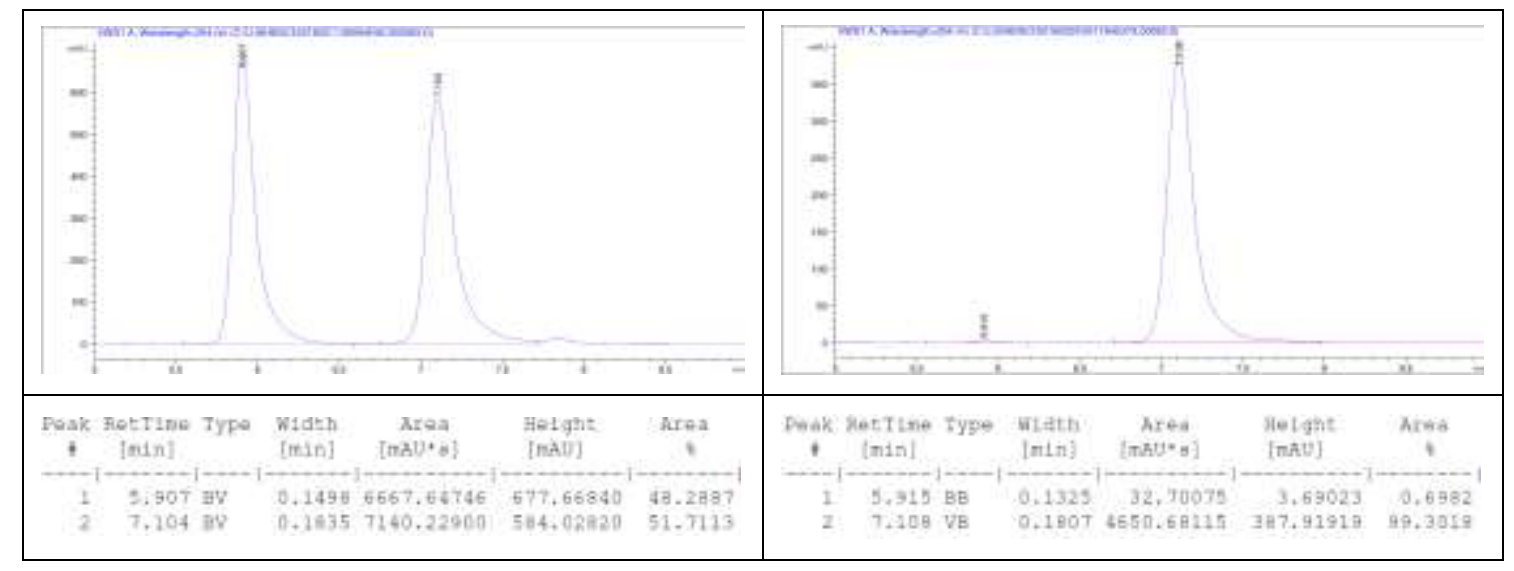

(S)-7-benzyl-2,11-dimethyl-9-tosyl-7,8-dihydrobenzo[2,3]oxocino[5,4-b]indol-6(9H)-one. 
(Scheme 5, 6l)

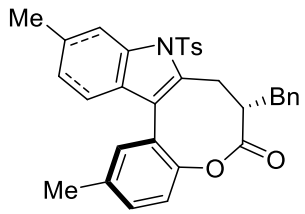

Used 2-Chloroaldehyde 2f. White solid, MP: $176-178{ }^{\circ} \mathrm{C} .{ }^{1} \mathbf{H}$ NMR $\left(500 \mathrm{MHz}, \mathrm{CDCl}_{3}\right): \delta$ $8.01(\mathrm{~s}, 1 \mathrm{H}), 7.68(\mathrm{~d}, J=8.0 \mathrm{~Hz}, 2 \mathrm{H}), 7.29-7.26(\mathrm{~m}, 4 \mathrm{H}), 7.24(\mathrm{~d}, J=7.0 \mathrm{~Hz}, 1 \mathrm{H}), 7.21(\mathrm{~s}$, $1 \mathrm{H}), 7.17(\mathrm{~d}, J=8.0 \mathrm{~Hz}, 1 \mathrm{H}), 7.13(\mathrm{~d}, J=8.0 \mathrm{~Hz}, 1 \mathrm{H}), 7.08(\mathrm{~d}, J=7.0 \mathrm{~Hz}, 2 \mathrm{H}), 7.04(\mathrm{~d}, J=$ $8.0 \mathrm{~Hz}, 1 \mathrm{H}), 6.83(\mathrm{~d}, J=8.0 \mathrm{~Hz}, 1 \mathrm{H}), 3.84(\mathrm{dd}, J=17.512 .5 \mathrm{~Hz}, 1 \mathrm{H}), 3.72-3.66(\mathrm{~m}, 1 \mathrm{H})$, 3.34 (dd, $J=17.55 .0 \mathrm{~Hz}, 1 \mathrm{H}), 3.15(\mathrm{dd}, J=13.59 .5 \mathrm{~Hz}, 1 \mathrm{H}), 2.91$ (dd, $J=13.55 .0 \mathrm{~Hz}, 1 \mathrm{H}$ ), 2.49 (s, 3H), 2.41 (s, 3H), 2.39 (s, 3H). ${ }^{13} \mathbf{C}$ NMR $\left(125 \mathrm{MHz}, \mathrm{CDCl}_{3}\right): \delta 173.53,147.32$, $145.01,138.19,136.94,135.85,135.33,132.91,132.73,131.58,130.20,130.11,128.66$, $128.52,127.87,126.67,126.40,125.53,125.19,120.89,119.05,117.20,114.85,39.81,39.22$, 33.37, 22.00, 21.61, 20.85. HRMS (ESI) $\mathrm{m} / \mathrm{z}$ Calcd for $\left[\mathrm{C}_{33} \mathrm{H}_{29} \mathrm{NNaO}_{4} \mathrm{~S}, \mathrm{M}+\mathrm{Na}\right]^{+}: 558.1709$; Found 558.1706.

Optical Rotation: $[\alpha]^{25}$ D $15.0\left(c=0.1, \mathrm{CHCl}_{3}\right) .98 \%$ ee (HPLC condition: Chiralpak IA column, $n$-Hexane $/ i-\mathrm{PrOH}=90: 10$, flow rate $=1.0 \mathrm{~mL} / \mathrm{min}$, wave length $=254 \mathrm{~nm}, t_{\mathrm{R}}=$ $8.67 \mathrm{~min}$ for minor isomer, $t_{\mathrm{R}}=11.51 \mathrm{~min}$ for major isomer).

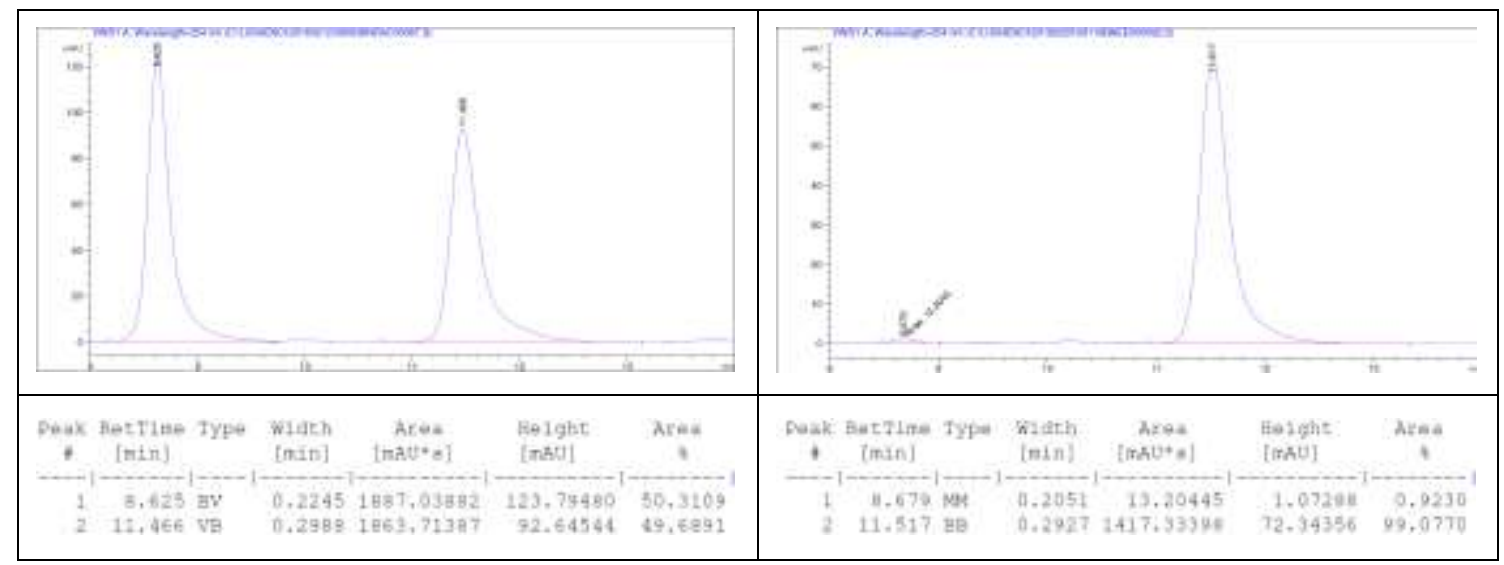

(S)-7-benzyl-2,11-dimethyl-9-tosyl-7,8-dihydrobenzo[2,3]oxocino[5,4-b]indol-6(9H)-one.

(Scheme 5, 6m)

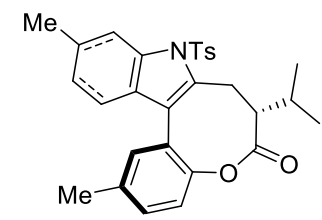

Used 2-Benzoylisovaleraldehyde 2a. White solid, MP: 167 - $168{ }^{\circ} \mathrm{C} .{ }^{1} \mathbf{H}$ NMR $(500 \mathrm{MHz}$, $\left.\mathrm{CDCl}_{3}\right): \delta 8.01(\mathrm{~s}, 1 \mathrm{H}), 7.69(\mathrm{~d}, J=8.5 \mathrm{~Hz}, 2 \mathrm{H}), 7.29-7.28(\mathrm{~m}, 2 \mathrm{H}), 7.24-7.22(\mathrm{~m}, 2 \mathrm{H}), 7.13$ $(\mathrm{d}, J=8.0 \mathrm{~Hz}, 1 \mathrm{H}), 7.09(\mathrm{~d}, J=8.0 \mathrm{~Hz}, 1 \mathrm{H}), 7.04(\mathrm{~d}, J=8.0 \mathrm{~Hz}, 1 \mathrm{H}), 3.68(\mathrm{dd}, J=17.513 .0$ $\mathrm{Hz}, 1 \mathrm{H}), 3.37$ (dd, $J=17.55 .0 \mathrm{~Hz}, 1 \mathrm{H}), 3.01-2.96(\mathrm{~m}, 1 \mathrm{H}), 2.49$ (s, 3H), $2.42(\mathrm{~s}, 3 \mathrm{H}), 2.38$ $(\mathrm{s}, 3 \mathrm{H}), 2.12-2.06(\mathrm{~m}, 1 \mathrm{H}), 0.97(\mathrm{~d}, J=7.0 \mathrm{~Hz}, 3 \mathrm{H}), 0.94(\mathrm{~d}, J=7.0 \mathrm{~Hz}, 3 \mathrm{H}) .{ }^{13} \mathbf{C}$ NMR $(125$ $\left.\mathrm{MHz}, \mathrm{CDCl}_{3}\right): \delta 174.40,147.67,144.98,136.94,135.90,135.69,135.21,133.76,132.82$, $130.30,130.08,127.93,126.44,125.65,125.11,120.64,119.01,116.92,114.78,45.62,32.01$, 
31.40, 22.00, 21.61, 21.31, 20.86, 19.63. HRMS (ESI) $\mathrm{m} / \mathrm{z}$ Calcd for $\left[\mathrm{C}_{29} \mathrm{H}_{29} \mathrm{NNaO}_{4} \mathrm{~S}, \mathrm{M}+\right.$ $\mathrm{Na}^{+}:$510.1709; Found 510.1706.

Optical Rotation: $[\alpha]^{25}$ - $45.0\left(c=0.1, \mathrm{CHCl}_{3}\right) .99 \%$ ee (HPLC condition: Chiralpak IA column, $n$-Hexane $/ i-\mathrm{PrOH}=90: 10$, flow rate $=1.0 \mathrm{~mL} / \mathrm{min}$, wave length $=254 \mathrm{~nm}, t_{\mathrm{R}}=$ $6.15 \mathrm{~min}$ for minor isomer, $t_{\mathrm{R}}=7.08 \mathrm{~min}$ for major isomer).

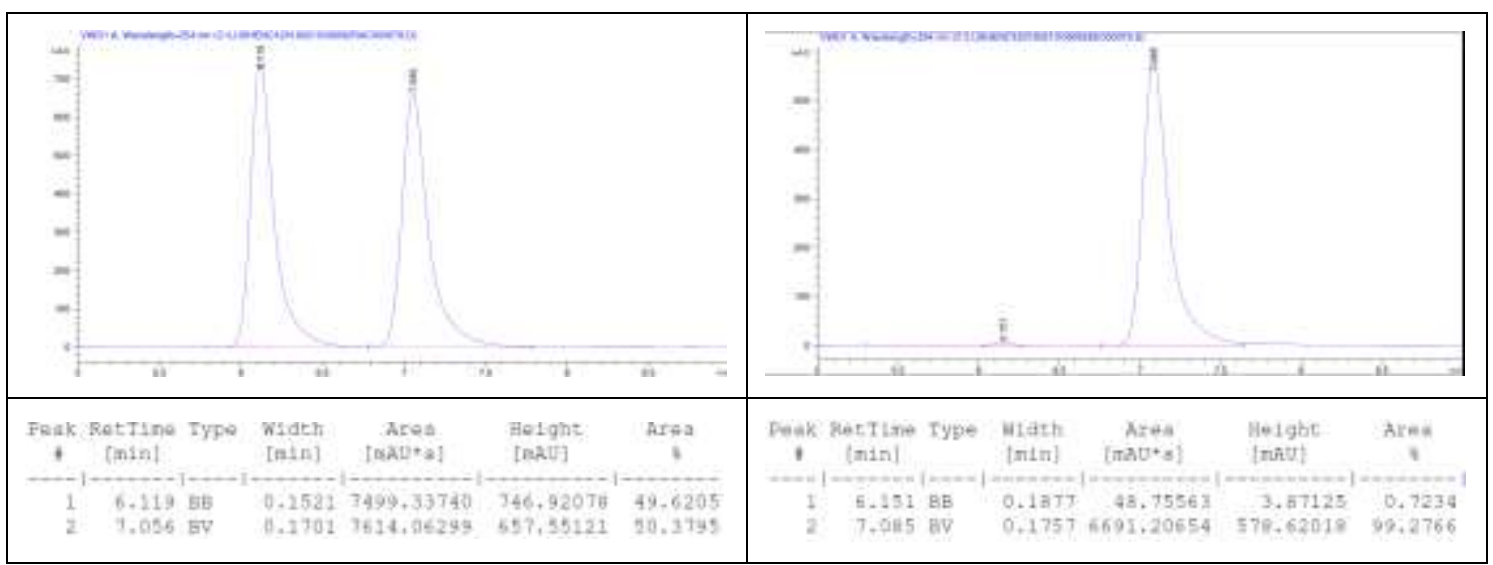

(S)-7-(3-((tert-butyldimethylsilyl)oxy)propyl)-2,11-dimethyl-9-tosyl-7,8-dihydrobenzo[2,3]oxocin o[5,4-b]indol-6(9H)-one. (Scheme 5, 6n)<smiles></smiles>

Used Enal 2g. Syrup. ${ }^{1} \mathbf{H}$ NMR (500 MHz, $\left.\mathrm{CDCl}_{3}\right): \delta 8.02(\mathrm{~s}, 1 \mathrm{H}), 7.67(\mathrm{~d}, J=8.5 \mathrm{~Hz}, 2 \mathrm{H})$, $7.28-7.27(\mathrm{~m}, 2 \mathrm{H}), 7.27-7.21(\mathrm{~m}, 2 \mathrm{H}), 7.14(\mathrm{~d}, J=8.0 \mathrm{~Hz}, 1 \mathrm{H}), 7.10(\mathrm{~d}, J=8.5 \mathrm{~Hz}, 1 \mathrm{H})$, $7.05(\mathrm{~d}, J=8.0 \mathrm{~Hz}, 1 \mathrm{H}), 3.67(\mathrm{dd}, J=17.512 .5 \mathrm{~Hz}, 1 \mathrm{H}), 3.55-3.50(\mathrm{~m}, 2 \mathrm{H}), 3.39-3.34(\mathrm{~m}$, $1 \mathrm{H}), 3.29(\mathrm{dd}, J=17.55 .0 \mathrm{~Hz}, 1 \mathrm{H}), 2.50(\mathrm{~s}, 3 \mathrm{H}), 2.41$ (s, 3H), $2.38(\mathrm{~s}, 3 \mathrm{H}), 1.89-1.84(\mathrm{~m}$, $1 \mathrm{H}), 1.69-1.64(\mathrm{~m}, 1 \mathrm{H}), 1.58-1.54(\mathrm{~m}, 1 \mathrm{H}), 1.40-1.36(\mathrm{~m}, 1 \mathrm{H}), 0.87(\mathrm{~s}, 9 \mathrm{H}), 0.02(\mathrm{~s}, 3 \mathrm{H})$, 0.001 (s, 3H). ${ }^{13} \mathbf{C}$ NMR (125 MHz, $\left.\mathrm{CDCl}_{3}\right): \delta 174.26,147.49,144.99,136.95,135.89$, $135.87,135.27,133.21,132.80,130.24,130.08,127.90,126.37,125.66,125.15,120.83$, 119.00, 117.09, 114.83, 62.19, 37.74, 33.64, 30.34, 29.83, 25.92, 22.00, 21.59, 20.82, 18.30 . -5.38, -5.42. HRMS (ESI) $\mathrm{m} / \mathrm{z}$ Calcd for $\left[\mathrm{C}_{35} \mathrm{H}_{43} \mathrm{NNaO}_{5} \mathrm{SSi}, \mathrm{M}+\mathrm{Na}\right]^{+}:$640.2523; Found 640.2525 .

Optical Rotation: $[\alpha]^{25}-76.0\left(c=0.2, \mathrm{CHCl}_{3}\right) .98 \%$ ee (HPLC condition: Chiralpak IA column, $n$-Hexane $/ i-\mathrm{PrOH}=90: 10$, flow rate $=1.0 \mathrm{~mL} / \mathrm{min}$, wave length $=254 \mathrm{~nm}, t_{\mathrm{R}}=$ $4.86 \mathrm{~min}$ for minor isomer, $t_{\mathrm{R}}=5.35 \mathrm{~min}$ for major isomer). 


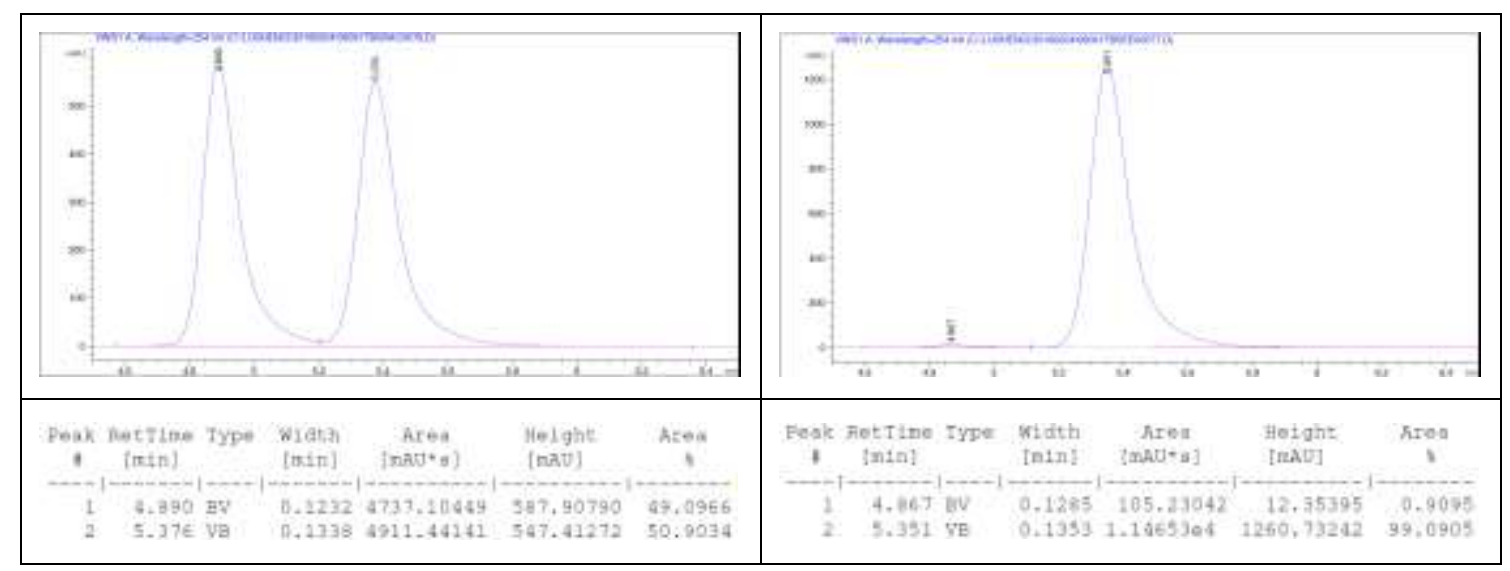




\section{The synthesis of substituted bridged biaryls and axially chiral biaryls}<smiles>Cc1cc([18OH])c2c(c1)OC(=O)C([18OH])Cc1oc3ccccc3c1-2</smiles>

Used Enal 2c. White solid. MP: 181-183. ${ }^{1} \mathbf{H}$ NMR (500 MHz, $\left.\mathrm{CDCl}_{3}\right): \delta 7.30(\mathrm{~s}, 1 \mathrm{H})$, 7.26-7.23 (m, 3H), $7.12-7.01(\mathrm{~m}, 5 \mathrm{H}), 6.79(\mathrm{t}, J=10.0 \mathrm{~Hz}, 1 \mathrm{H}), 6.66(\mathrm{~d}, J=10.0 \mathrm{~Hz}, 1 \mathrm{H})$, 3.50-3.40 (m, 1H), 3.39-3.36 (m, 1H), 3.17-3.11 (m, 1H), $2.52(\mathrm{~s}, 3 \mathrm{H}), 2.01-1.99(\mathrm{~m}, 1 \mathrm{H})$, 1.65-1.64 (m, 1H), 1.45-1.27 (m, 4H), $0.94(\mathrm{t}, J=10.0 \mathrm{~Hz}, 3 \mathrm{H}) .{ }^{13} \mathbf{C} \mathbf{~ N M R}\left(125 \mathrm{MHz}, \mathrm{CDCl}_{3}\right)$ $\delta 174.03,153.85,151.91,150.46,143.68,140.41,140.08,129.35,128.73,127.87,127.69$, $127.16,123.73,121.96,120.72,120.18,120.16,111.88,110.31,38.85,33.00,32.73,29.72$, 22.55, 21.21, 13.90. HRMS (ESI) $\mathrm{m} / \mathrm{z}$ Calcd for $\left[\mathrm{C}_{28} \mathrm{H}_{26} \mathrm{NaO}_{3}, \mathrm{M}+\mathrm{Na}\right]^{+}$: 433.1724; Found 433.1728 .

Optical Rotation: $[\alpha]^{25}$ D $15.0\left(c=0.1, \mathrm{CHCl}_{3}\right) .86 \%$ ee (HPLC condition: Chiralpak ID column, $n$-Hexane $/ i-\mathrm{PrOH}=90: 10$, flow rate $=1.0 \mathrm{~mL} / \mathrm{min}$, wave length $=254 \mathrm{~nm}, t_{\mathrm{R}}=$ $6.98 \mathrm{~min}$ for minor isomer, $t_{\mathrm{R}}=18.91 \mathrm{~min}$ for major isomer).

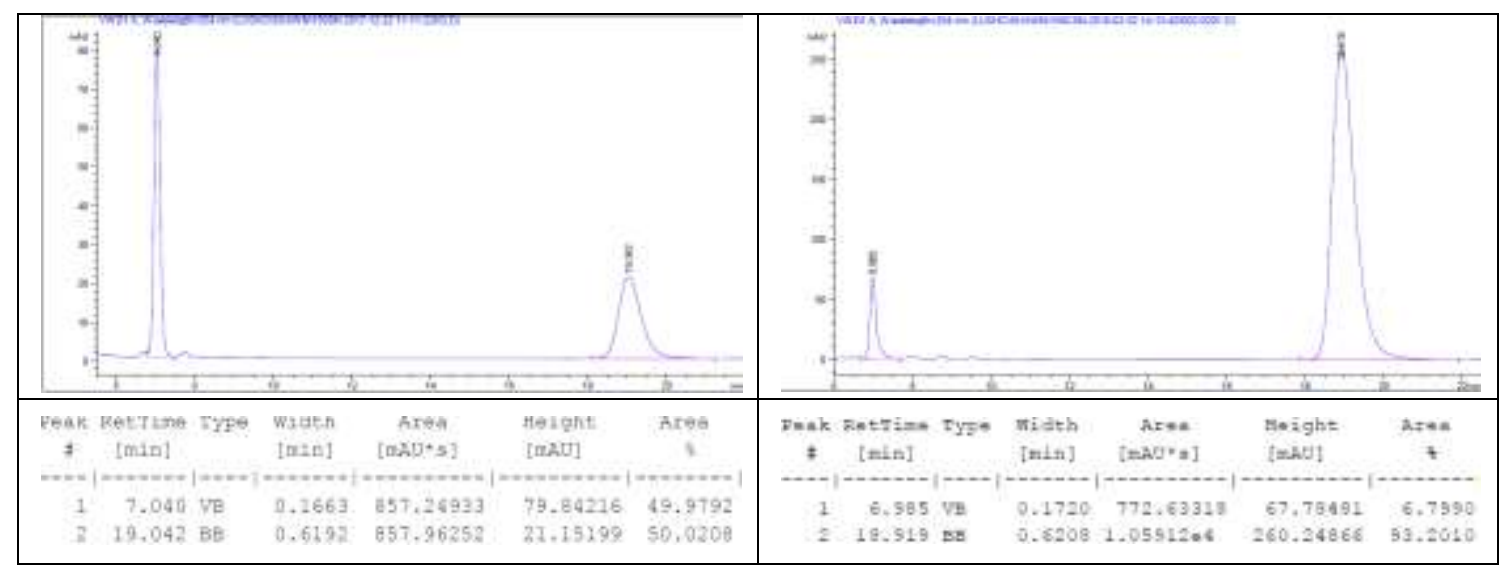

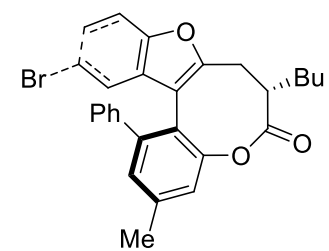

Used Enal 2c. White solid. MP: 201-202. ${ }^{1} \mathbf{H}$ NMR (500 MHz, $\mathrm{CDCl}_{3}$ ): $\delta 7.30$ (s, 1H), 7.22-7.21 (m, 2H), $7.16-7.09(\mathrm{~m}, 6 \mathrm{H}), 6.76(\mathrm{~s}, 1 \mathrm{H}), 3.47-3.43(\mathrm{~m}, 1 \mathrm{H}), 3.35(\mathrm{dd}, J=20.0$ $10.0 \mathrm{~Hz}, 1 \mathrm{H}), 3.12(\mathrm{dd}, J=15.014 .0 \mathrm{~Hz}, 1 \mathrm{H}), 2.52(\mathrm{~s}, 3 \mathrm{H}), 2.00-1.97(\mathrm{~m}, 1 \mathrm{H}), 1.65-1.59(\mathrm{~m}$, 2H), 1.41-1.26 (m, 4H), $0.93(\mathrm{t}, J=10.0 \mathrm{~Hz}, 3 \mathrm{H}) .{ }^{13} \mathbf{C}$ NMR $\left(125 \mathrm{MHz}, \mathrm{CDCl}_{3}\right): \delta 173.80$, $153.36,152.56,150.35,143.66,140.49,140.13,129.50,129.34,128.72,127.94,127.44$, 126.59, 123.04, 120.79, 119.49, 115.17, 111.79, 111.58, 38.70, 32.97, 32.68, 29.67, 22.52, 21.21, 13.87. HRMS (ESI) $\mathrm{m} / \mathrm{z}$ Calcd for $\left[\mathrm{C}_{28} \mathrm{H}_{25} \mathrm{BrNaO}_{3}, \mathrm{M}+\mathrm{Na}\right]^{+}:$511.0879; Found 511.0875 . 
Optical Rotation: $[\alpha]^{25} \mathrm{D}-27.0\left(c=0.1, \mathrm{CHCl}_{3}\right) .81 \%$ ee (HPLC condition: Chiralpak IC column, $n$-Hexane $/ i-\mathrm{PrOH}=99: 1$, flow rate $=1.0 \mathrm{~mL} / \mathrm{min}$, wave length $=254 \mathrm{~nm}, t_{\mathrm{R}}=$ $14.13 \mathrm{~min}$ for major isomer, $t_{\mathrm{R}}=20.73 \mathrm{~min}$ for minor isomer).

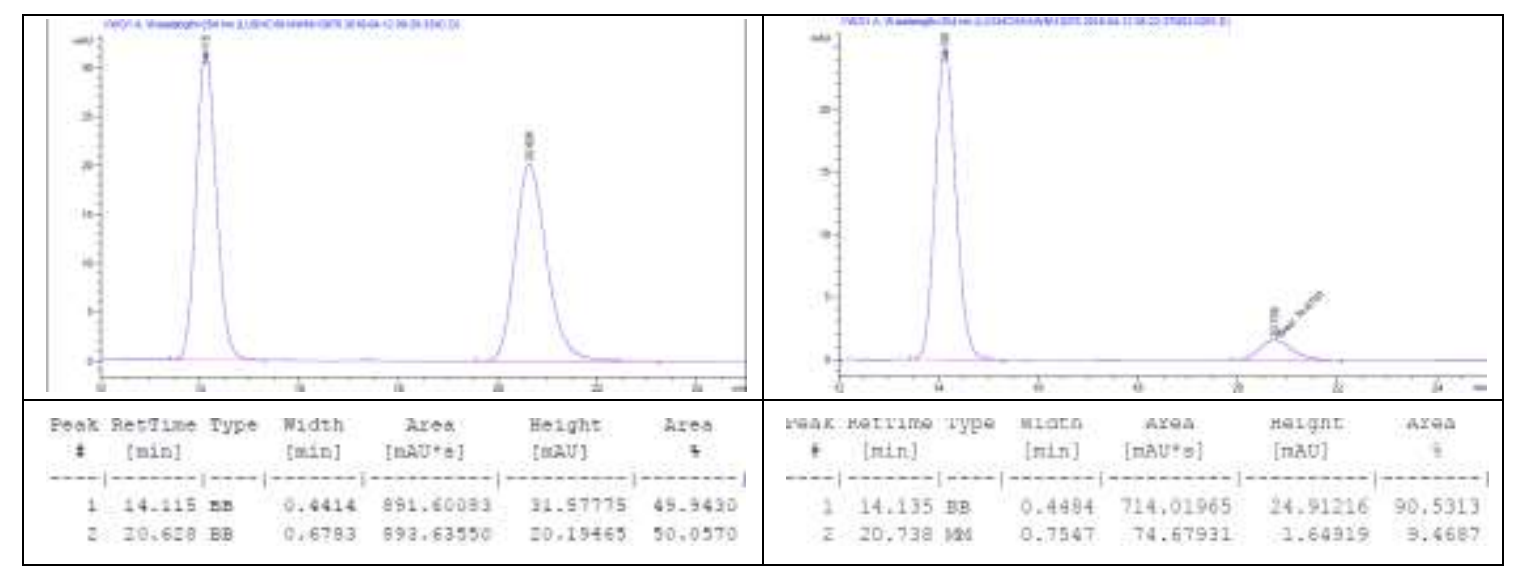

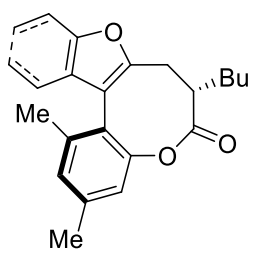

Used Enal 2c. Syrup. ${ }^{1} \mathbf{H}$ NMR $\left(500 \mathrm{MHz}, \mathrm{CDCl}_{3}\right): \delta 7.37-7.34(\mathrm{~m}, 1 \mathrm{H}), 7.18(\operatorname{td}, J=10.0$ $5.0 \mathrm{~Hz}, 1 \mathrm{H}), 7.10(\mathrm{td}, J=10.05 .0 \mathrm{~Hz}, 1 \mathrm{H}), 7.06(\mathrm{~d}, J=5.0 \mathrm{~Hz}, 1 \mathrm{H}), 7.01(\mathrm{~s}, 1 \mathrm{H}), 3.15-3.07$ (m, 2H), 2.99-2.94 (m, 1H), $2.33(\mathrm{~s}, 3 \mathrm{H}), 2.10(\mathrm{~s}, 3 \mathrm{H}), 1.81-1.75(\mathrm{~m}, 1 \mathrm{H}), 1.47-1.42(\mathrm{~m}, 1 \mathrm{H})$, $1.25-1.12(\mathrm{~m}, 1 \mathrm{H}), 0.80(\mathrm{t}, J=10.0 \mathrm{~Hz}, 3 \mathrm{H}) .{ }^{13} \mathbf{C} \mathbf{N M R}\left(125 \mathrm{MHz}, \mathrm{CDCl}_{3}\right): \delta 174.28,154.16$, $151.91,150.23,139.62,129.23,128.55,124.17,122.69,121.08,120.23,119.06,111.44$, 111.15, 38.67, 32.82, 32.69, 29.63, 22.46, 21.09, 20.72, 13.85. HRMS (ESI) m/z Calcd for $\left[\mathrm{C}_{23} \mathrm{H}_{24} \mathrm{NaO}_{3}, \mathrm{M}+\mathrm{Na}\right]^{+}:$371.1617; Found 371.1614.

Optical Rotation: $[\alpha]^{25}$ - $12.0\left(c=0.1, \mathrm{CHCl}_{3}\right) .91 \%$ ee (HPLC condition: Chiralpak ID column, $n$-Hexane $/ i-\mathrm{PrOH}=90: 10$, flow rate $=1.0 \mathrm{~mL} / \mathrm{min}$, wave length $=254 \mathrm{~nm}, t_{\mathrm{R}}=$ $6.79 \mathrm{~min}$ for minor isomer, $t_{\mathrm{R}}=19.82 \mathrm{~min}$ for major isomer).

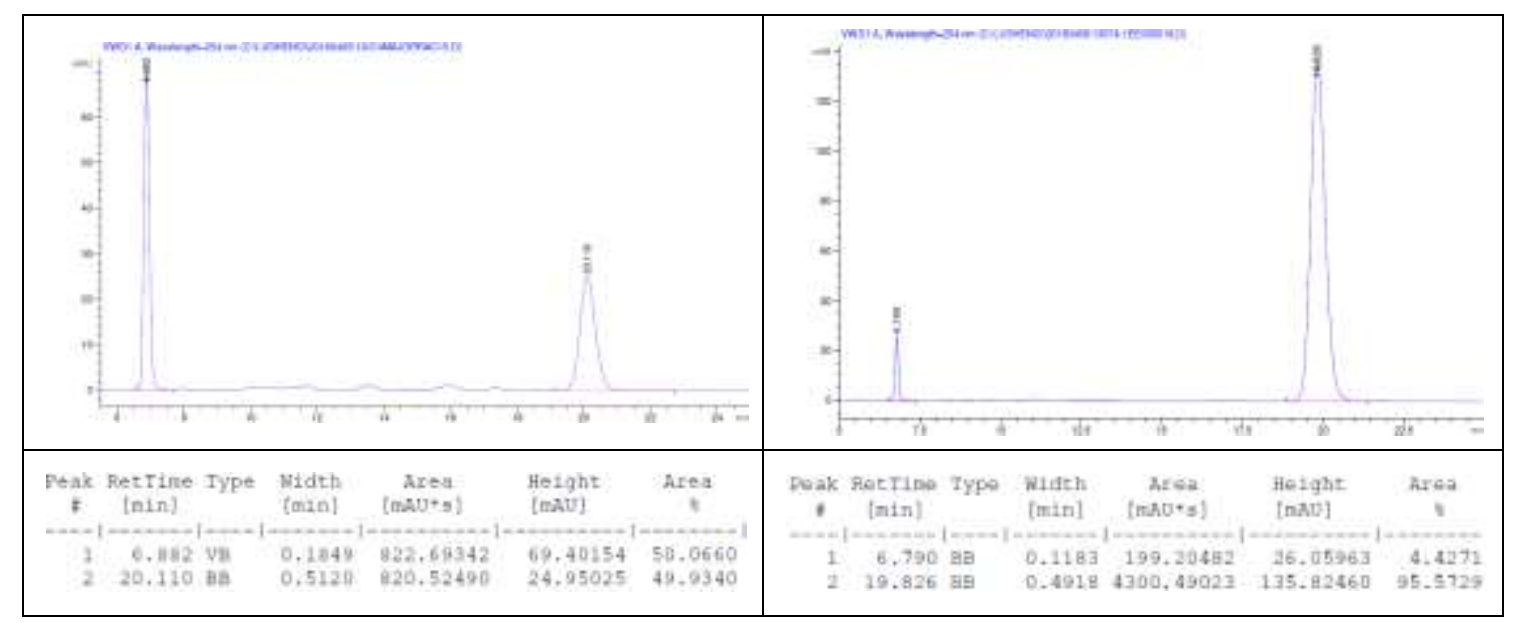




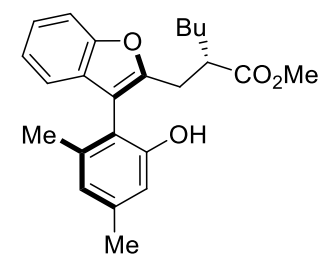

Syrup. ${ }^{1} \mathbf{H}$ NMR $\left(500 \mathrm{MHz}, \mathrm{CDCl}_{3}\right): \delta$ 7.52-7.50 (m, 1H), 7.33-7.30 (m, 1H), 7.23 -7.21 (m, $2 \mathrm{H}), 6.75(\mathrm{~d}, J=10.0 \mathrm{~Hz}, 1 \mathrm{H}), 4.98(\mathrm{~s}, 1 \mathrm{H}), 3.63(\mathrm{~s}, 3 \mathrm{H}), 3.01-3.00(\mathrm{~m}, 1 \mathrm{H}), 2.87-2.81(\mathrm{~m}$, $1 \mathrm{H}), 2.38(\mathrm{~s}, 3 \mathrm{H}), 2.05(\mathrm{~s}, 3 \mathrm{H}), 1.67-1.66(\mathrm{~m}, 1 \mathrm{H}), 1.54-1.51(\mathrm{~m}, 1 \mathrm{H}), 1.31-1.20(\mathrm{~m}, 4 \mathrm{H}), 0.87$ $(\mathrm{t}, J=10.0 \mathrm{~Hz}, 3 \mathrm{H}) .{ }^{13} \mathrm{C}$ NMR $\left(125 \mathrm{MHz}, \mathrm{CDCl}_{3}\right): \delta 175.34,154.70,154.34,154.01,139.51$, $138.82,128.62$, 124.31, 123.06, 122.98, 119.97, 113.77, 113.36, 111.16, 110.83, 51.75, 43.95, $31.62,29.41,29.19,22.49,21.34,20.00,13.86$. HRMS (ESI) m/z Calcd for $\left[\mathrm{C}_{24} \mathrm{H}_{28} \mathrm{NaO}_{4}, \mathrm{M}\right.$ $+\mathrm{Na}]^{+}:$403.1880; Found 403.1884.

Optical Rotation: $[\alpha]^{25}$ - $23.0\left(c=0.1, \mathrm{CHCl}_{3}\right) .91 \%$ ee (HPLC condition: Chiralpak IC column, $n$-Hexane $/ i-\mathrm{PrOH}=95: 5$, flow rate $=1.0 \mathrm{~mL} / \mathrm{min}$, wave length $=254 \mathrm{~nm}, t_{\mathrm{R}}=$ $5.43 \mathrm{~min}$ for major isomer, $t_{\mathrm{R}}=7.29 \mathrm{~min}$ for minor isomer).

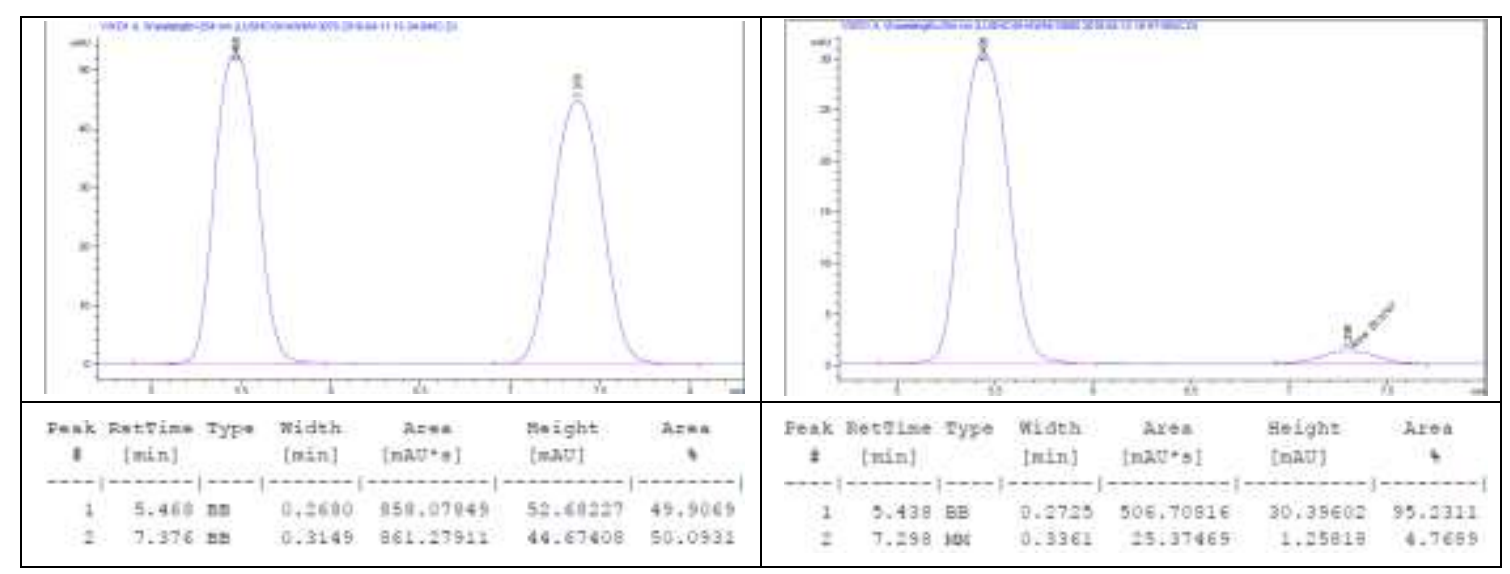




\section{Crystal structures of $4 b, 6 g$ and $4 u$}

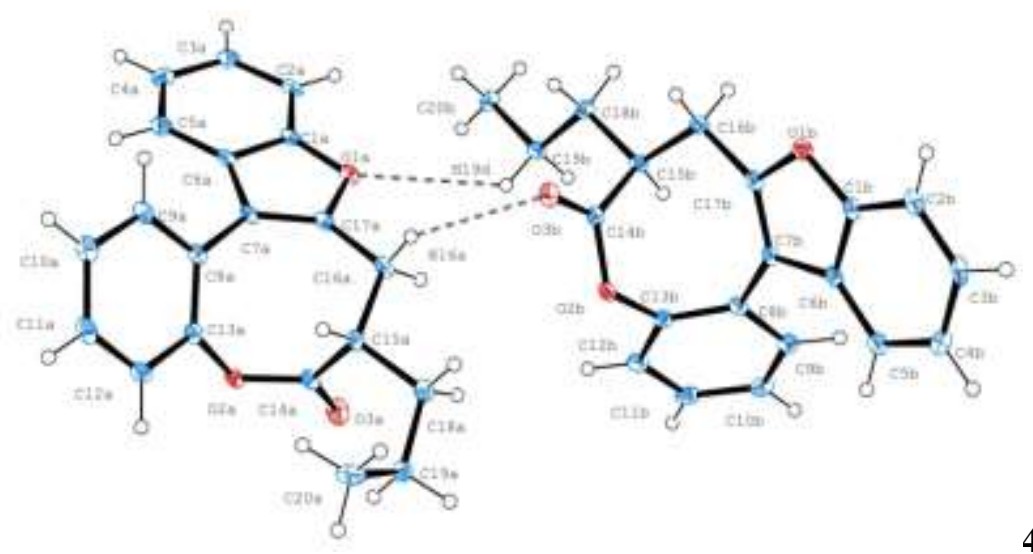

4b

Identification code

Empirical formula

Formula weight

Temperature

Wavelength

Crystal system

Space group

Unit cell dimensions

Volume

$\mathrm{Z}$

Density (calculated)

Absorption coefficient

$\mathrm{F}(000)$

Crystal size

Theta range for data collection

Index ranges

Reflections collected

Independent reflections

Completeness to theta $=67.679^{\circ}$

Refinement method

Data / restraints / parameters

Goodness-of-fit on $\mathrm{F}^{2}$

Final R indices [I $>2 \operatorname{sigma}(\mathrm{I})]$ 4b

$\mathrm{C}_{40} \mathrm{H}_{36} \mathrm{O}_{6}$

612.69

100(2) K

$1.54178 \AA$

Monoclinic

P 2(1)

$$
\begin{array}{ll}
\mathrm{a}=11.9855(13) \AA & \alpha=90^{\circ} \\
\mathrm{b}=7.8837(9) \AA & \beta=90.079(2)^{\circ} \\
\mathrm{c}=16.3210(18) \AA & \gamma=90^{\circ}
\end{array}
$$

1542.2(3) $\AA^{3}$

2

$1.319 \mathrm{Mg} / \mathrm{m}^{3}$

$0.706 \mathrm{~mm}^{-1}$

648

$0.561 \times 0.488 \times 0.206 \mathrm{~mm}^{3}$

2.707 to $74.669^{\circ}$

$-7<=\mathrm{h}<=14,-8<=\mathrm{k}<=9,-19<=1<=20$

10098

$5199[\mathrm{R}(\mathrm{int})=0.0311]$

$98.4 \%$

Full-matrix least-squares on $\mathrm{F}^{2}$

5199 / 1 / 419

1.072

$\mathrm{R} 1=0.0327, \mathrm{wR} 2=0.0828$ 
$\mathrm{R}$ indices (all data)

Absolute structure parameter

Extinction coefficient

Largest diff. peak and hole
$\mathrm{R} 1=0.0330, \mathrm{wR} 2=0.0870$

$-0.08(7)$

$0.0004(8)$

0.256 and -0.253 e. $\AA^{-3}$

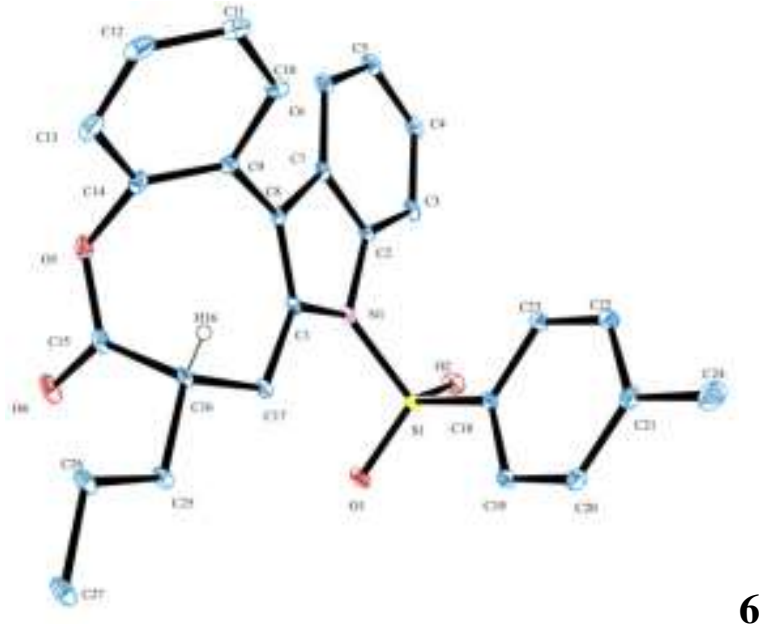

Identification code

Empirical formula

Formula weight

Temperature

Wavelength

Crystal system

Space group

Unit cell dimensions

Volume

Z

Density (calculated)

Absorption coefficient

$\mathrm{F}(000)$

Crystal size

Theta range for data collection

Index ranges

Reflections collected

Independent reflections

Completeness to theta $=67.679^{\circ}$
$6 \mathrm{~g}$

\section{$\mathrm{C}_{27} \mathrm{H}_{25} \mathrm{NO}_{4} \mathrm{~S}$}

459.54

100(2) K

$1.54178 \AA$

Monoclinic

P 2(1)

$$
\begin{array}{ll}
\mathrm{a}=8.1622(4) \AA & \alpha=90^{\circ} \\
\mathrm{b}=7.4452(3) \AA & \beta=93.3480(10)^{\circ} \\
\mathrm{c}=18.6526(8) \AA & \gamma=90^{\circ}
\end{array}
$$

1131.57(9) $\AA^{3}$

2

$1.349 \mathrm{Mg} / \mathrm{m}^{3}$

$1.556 \mathrm{~mm}^{-1}$

484

$0.554 \times 0.090 \times 0.057 \mathrm{~mm}^{3}$

2.373 to $72.369^{\circ}$

$-10<=\mathrm{h}<=10,-9<=\mathrm{k}<=8,-22<=\mathrm{l}<=23$

23933

$4390[\mathrm{R}($ int $)=0.0352]$

$100.0 \%$ 
Refinement method

Data / restraints / parameters

Goodness-of-fit on $\mathrm{F}^{2}$

Final $\mathrm{R}$ indices [I $>2 \operatorname{sigma}(\mathrm{I})]$

$\mathrm{R}$ indices (all data)

Absolute structure parameter

Largest diff. peak and hole
Full-matrix least-squares on $\mathrm{F}^{2}$

$4390 / 1 / 300$

1.061

$\mathrm{R} 1=0.0254, w \mathrm{R} 2=0.0637$

$\mathrm{R} 1=0.0259, \mathrm{wR} 2=0.0641$

$0.082(5)^{*}$

0.185 and -0.304 e. $\AA^{-3}$

*Flack x determined using 1912 quotients [(I+)-(I-)]/[(I+)+(I-)]

(Parsons, Flack and Wagner, Acta Cryst. B69 (2013) 249-259).

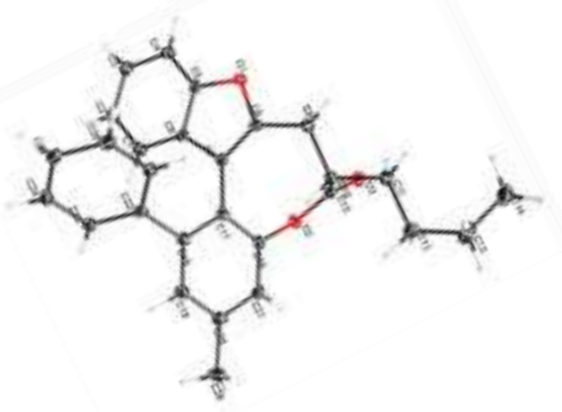

$4 u$

Identification code

Empirical formula

Formula weight

Temperature

Wavelength

Crystal system

Space group

Unit cell dimensions

Volume

$\mathrm{Z}$

Density (calculated)

Absorption coefficient

$\mathrm{F}(000)$

Crystal size

\section{$4 u$}

C28 H26 O3

410.49

100(2) K

$1.54178 \AA$

Monoclinic

P21

$$
\begin{array}{ll}
\mathrm{a}=7.9247(4) \AA & \alpha=90^{\circ} . \\
\mathrm{b}=10.0883(5) \AA & \beta=99.2020(10)^{\circ} . \\
\mathrm{c}=13.7799(7) \AA & \gamma=90^{\circ} .
\end{array}
$$

1087.48(9) $\AA^{3}$

2

$1.254 \mathrm{Mg} / \mathrm{m}^{3}$

$0.634 \mathrm{~mm}^{-1}$

436

$0.230 \times 0.200 \times ? 0.153 \mathrm{~mm}^{3}$ 
Theta range for data collection

Index ranges

Reflections collected

Independent reflections

Completeness to theta $=67.679^{\circ}$

Absorption correction

Max. and min. transmission

Refinement method

Data / restraints / parameters

Goodness-of-fit on $\mathrm{F}^{2}$

Final R indices [I>2sigma(I)]

$\mathrm{R}$ indices (all data)

Absolute structure parameter

Extinction coefficient

Largest diff. peak and hole
3.249 to $70.152^{\circ}$.

$-9<=\mathrm{h}<=9,-12<=\mathrm{k}<=12,-16<=1<=16$

71472

$4127[\mathrm{R}(\mathrm{int})=0.0265]$

$100.0 \%$

Semi-empirical from equivalents

0.7533 and 0.7106

Full-matrix least-squares on $\mathrm{F}^{2}$

4127 / 1 / 283

1.020

$\mathrm{R} 1=0.0244, \mathrm{wR} 2=0.0668$

$\mathrm{R} 1=0.0245, \mathrm{wR} 2=0.0669$

$0.06(2)$

$0.0123(10)$

0.205 and -0.181 e. $\AA^{-3}$ 


\section{Investigation of side reaction pathway}

There is a competitive reaction pathway for our catalytic reactions, which contributed to the low to moderate yields of our products. We were able to isolate and characterize the side products of the reaction to be tetracyclic indole- or benzofuran structures $\mathbf{9}$ and $\mathbf{1 1}$ as shown below. We later discovered that substrates $\mathbf{1}$ or $\mathbf{5}$ could undergo intramolecular cyclization simply in the presence of DIPEA to deliver $\mathbf{8}$ or the analogous $\mathbf{1 0}$. These intermediates can produce $\mathbf{9}$ or $\mathbf{1 1}$ exclusively under NHC catalysis.

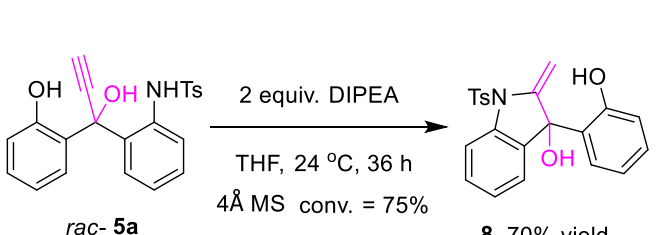

$8,70 \%$ yield
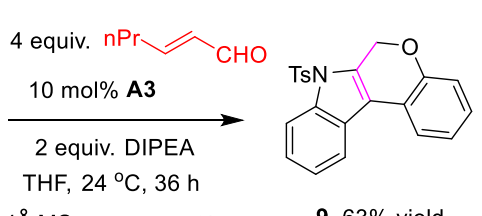

$4 \AA \mathrm{MS}$ conv. $=70 \%$
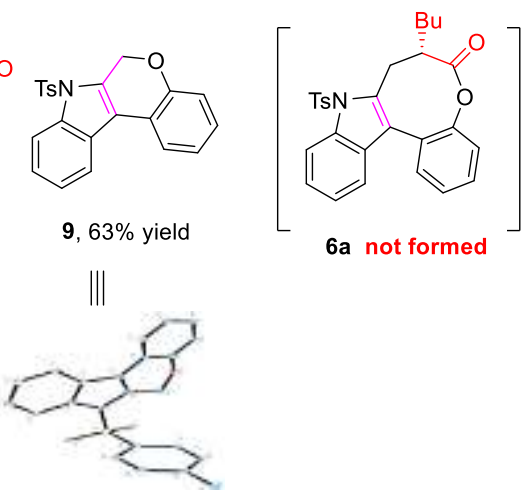

6a not formed
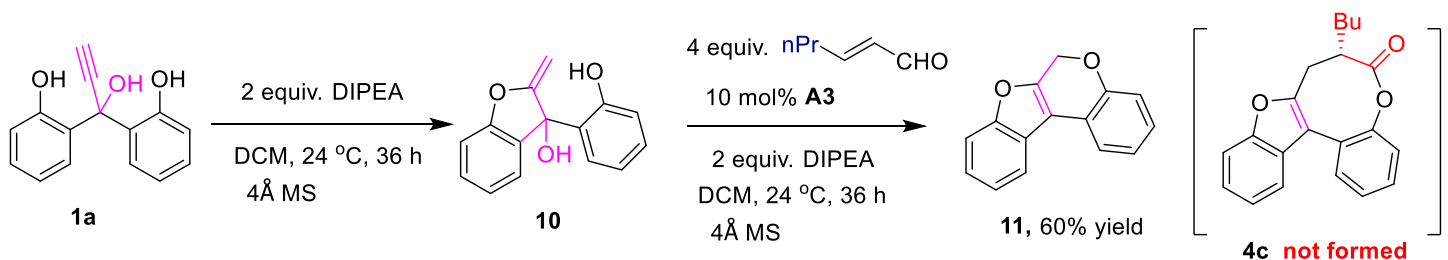

3-(2-hydroxyphenyl)-2-methylene-1-tosylindolin-3-ol (8).

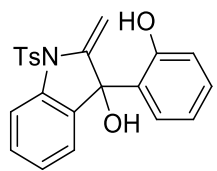

Syrup. ${ }^{1}$ H NMR $\left(500 \mathrm{MHz}, \mathrm{CDCl}_{3}\right): \delta 8.95(\mathrm{~s}, 1 \mathrm{H}), 7.98(\mathrm{~d}, J=8.5 \mathrm{~Hz}, 1 \mathrm{H}), 7.62(\mathrm{~d}, J=8.5$ $\mathrm{Hz}, 2 \mathrm{H}), 7.43(\mathrm{td}, J=7.51 .0 \mathrm{~Hz}, 1 \mathrm{H}), 7.33(\mathrm{~d}, J=7.5 \mathrm{~Hz}, 1 \mathrm{H}), 7.21-7.15(\mathrm{~m}, 3 \mathrm{H}), 7.07$ (td, $J=7.51 .0 \mathrm{~Hz}, 1 \mathrm{H}), 6.85(\mathrm{~d}, J=8.5 \mathrm{~Hz}, 1 \mathrm{H}), 6.36(\mathrm{t}, J=7.5 \mathrm{~Hz}, 1 \mathrm{H}), 5.98(\mathrm{~s}, 1 \mathrm{H}), 5.81(\mathrm{~d}, J$ $=8.5 \mathrm{~Hz}, 1 \mathrm{H}), 5.27(\mathrm{~d}, J=2.0 \mathrm{~Hz}, 1 \mathrm{H}), 2.96(\mathrm{~s}, 1 \mathrm{H}), 2.40(\mathrm{~s}, 3 \mathrm{H}) .{ }^{13} \mathbf{C}$ NMR $(125 \mathrm{MHz}$, $\left.\mathrm{CDCl}_{3}\right): \delta 155.20,151.96,145.04,140.49,134.11,133.79,130.88,129.65,129.19,128.46$, $127.41,126.15,125.78,125.32,119.35,117.91,116.43,102.32,84.28,21.59$. HRMS (ESI) $\mathrm{m} / \mathrm{z}$ Calcd for $\left[\mathrm{C}_{22} \mathrm{H}_{19} \mathrm{NNaO}_{4} \mathrm{~S}, \mathrm{M}+\mathrm{Na}\right]^{+}$: 416.0927; Found 416.0928. 
7-tosyl-6,7-dihydrochromeno[3,4-b]indole (Scheme 5b, 9)

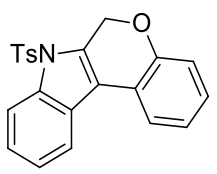

White solid, MP: $130-132{ }^{\circ} \mathrm{C} .{ }^{1} \mathbf{H}$ NMR $\left(500 \mathrm{MHz}, \mathrm{CDCl}_{3}\right): \delta 8.20(\mathrm{dd}, J=7.52 .0 \mathrm{~Hz}, 1 \mathrm{H})$, $7.94(\mathrm{dd}, J=7.52 .0 \mathrm{~Hz}, 1 \mathrm{H}), 7.76(\mathrm{dd}, J=7.51 .5 \mathrm{~Hz}, 1 \mathrm{H}), 7.72(\mathrm{~d}, J=8.5 \mathrm{~Hz}, 2 \mathrm{H}), 7.41-$ $7.31(\mathrm{~m}, 2 \mathrm{H}), 7.24(\mathrm{~d}, J=8.5 \mathrm{~Hz}, 2 \mathrm{H}), 7.20(\mathrm{td}, J=7.52 .0 \mathrm{~Hz}, 1 \mathrm{H}), 7.07(\mathrm{td}, J=7.51 .0 \mathrm{~Hz}$, $1 \mathrm{H}), 7.03(\mathrm{~d}, J=8.0 \mathrm{~Hz}, 1 \mathrm{H}), 5.76(\mathrm{~s}, 2 \mathrm{H}), 2.35(\mathrm{~s}, 3 \mathrm{H}) .{ }^{13} \mathrm{C} \mathbf{~ N M R}\left(125 \mathrm{MHz}, \mathrm{CDCl}_{3}\right): \delta$ $152.22,145.35,136.92,135.07,133.31,130.09,130.02,128.06,126.58,124.83,124.33$, 123.41, 122.07, 120.31, 120.21, 116.54, 114.62, 114.31, 64.19, 21.57. HRMS (ESI) m/z Calcd for $\left[\mathrm{C}_{22} \mathrm{H}_{17} \mathrm{NNaO}_{3} \mathrm{~S}, \mathrm{M}+\mathrm{Na}\right]^{+}:$398.0821; Found 398.0820.

6H-benzofuro[2,3-c]chromene (11)

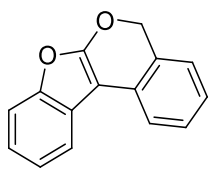

Syrup. ${ }^{1} \mathbf{H}$ NMR $\left(500 \mathrm{MHz}, \mathrm{CDCl}_{3}\right): \delta 7.92(\mathrm{~d}, J=7.0 \mathrm{~Hz}, 1 \mathrm{H}), 7.69(\mathrm{~d}, J=7.5 \mathrm{~Hz}, 1 \mathrm{H}), 7.55$ $(\mathrm{dd}, J=7.01 .5 \mathrm{~Hz}, 1 \mathrm{H}), 7.39-7.34(\mathrm{~m}, 2 \mathrm{H}), 7.18(\mathrm{t}, J=7.5 \mathrm{~Hz}, 1 \mathrm{H}), 7.09$ (t, $J=7.5 \mathrm{~Hz}, 1 \mathrm{H})$, $7.01(\mathrm{~d}, J=7.5 \mathrm{~Hz}, 1 \mathrm{H}), 5.52(\mathrm{~s}, 2 \mathrm{H}) .{ }^{13} \mathbf{C} \mathbf{N M R}\left(125 \mathrm{MHz}, \mathrm{CDCl}_{3}\right): \delta 155.60,151.78,148.29$, $127.65,124.28,124.00,123.61,123.17,122.07,120.12,117.71,116.38,112.04,111.80$, 63.79. HRMS (ESI) $\mathrm{m} / \mathrm{z}$ Calcd for $\left[\mathrm{C}_{15} \mathrm{H}_{10} \mathrm{NaO}_{2}, \mathrm{M}+\mathrm{Na}\right]^{+}:$245.0573; Found 245.0577. 


\section{Reference.}

1. Kerr, M. S.; Alaniz, J. R. D.; Rovis, T. J. Org. Chem. 2005, 70, 5725 - 5728.

2. He, M.; Struble, J. R.; Bode, J. W. J. Am. Chem. Soc. 2006, 128, $8418-8420$.

3. Ling, K. B.; Smith, A. D. Chem. Commun. 2011, 47, 373 - 375.

4. Shan, G.; Han, X.; Lin, Y.; Yu, S.; Rao, Y. Org. Biomol. Chem. 2013, 11, 2318-2322.

5. Li, H.; Li, X.; Wang, H.-Y.; Winston-McPherson, G. N.; Geng, H.-M. J.; Guzei, I. A.; Tang, W. Chem. Commun. 2014, 50, 12293-12296.

6. Jing, Q.; Sandoval, C. A.; Wang, Z.; Ding, K. Eur. J. Org. Chem. 2006, 3606-3616.

7. Gardikis, Y.; Tsoungas, P. G.; Potamitis, C.; Zervou, M.; Cordopatis, P. Heterocycles, 2011, 83, 1077-1091. 


\section{NMR spectra}

$(M, S)$-7-isopropyl-7,8-dihydro-6H-benzo[2,3]oxocino[5,4-b]benzofuran-6-one. (Scheme 3, 4a)
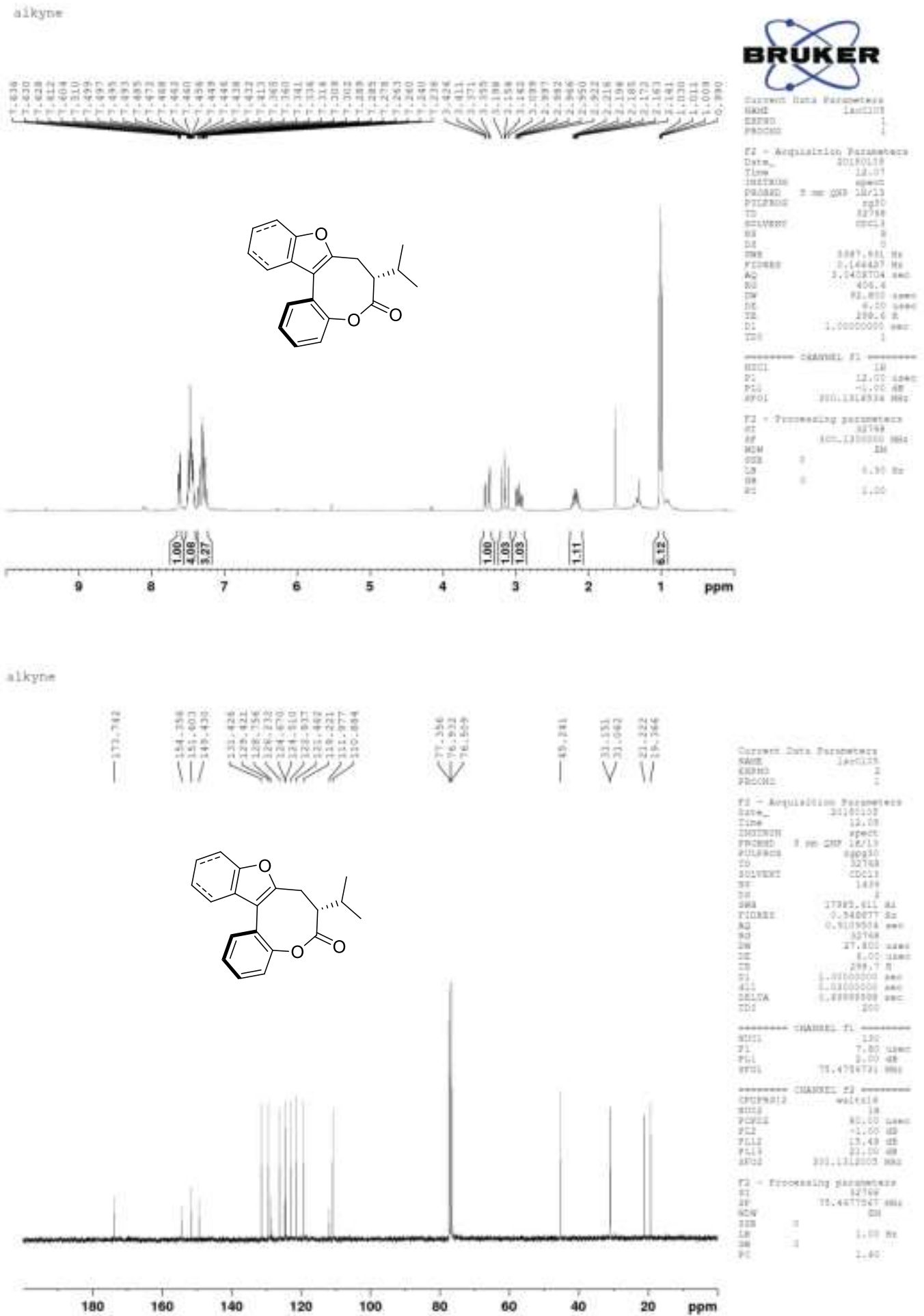
( $M, S$ )-7-propyl-7,8-dihydro-6H-benzo[2,3] oxocino[5,4-b]benzofuran-6-one. (Scheme 4a, 4b)

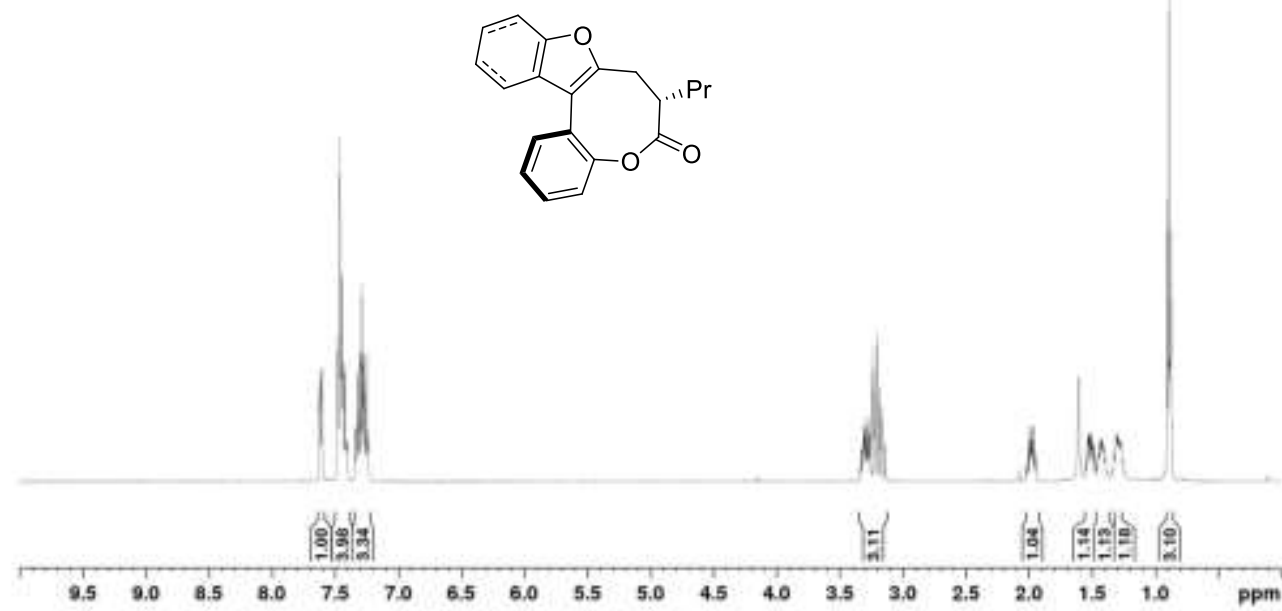

propre 1 a ring

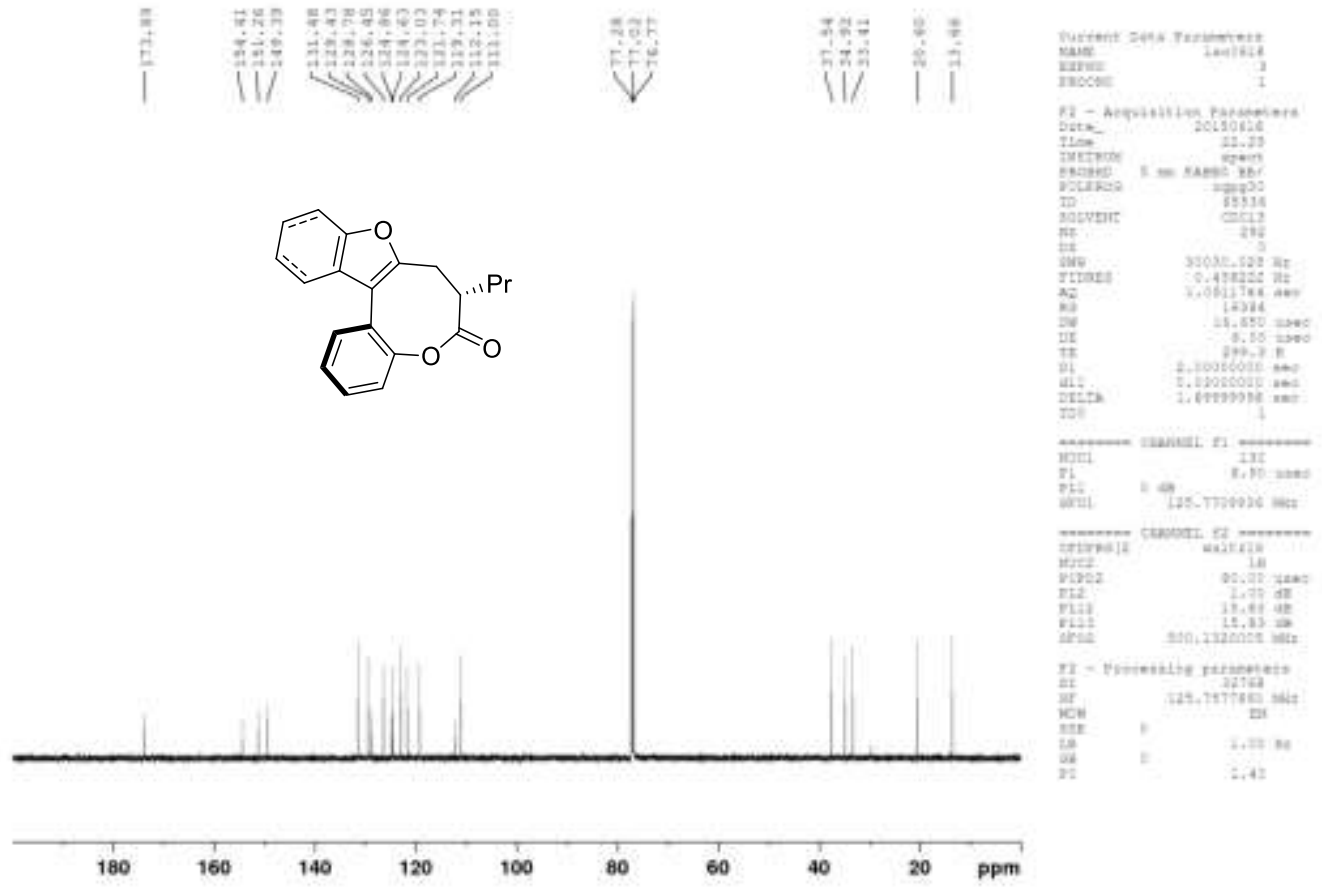


(M, S)-7-butyl-7,8-dihydro-6H-benzo[2,3] oxocino[5,4-b]benzofuran-6-one. (Scheme 4a, 4c) buty1 8
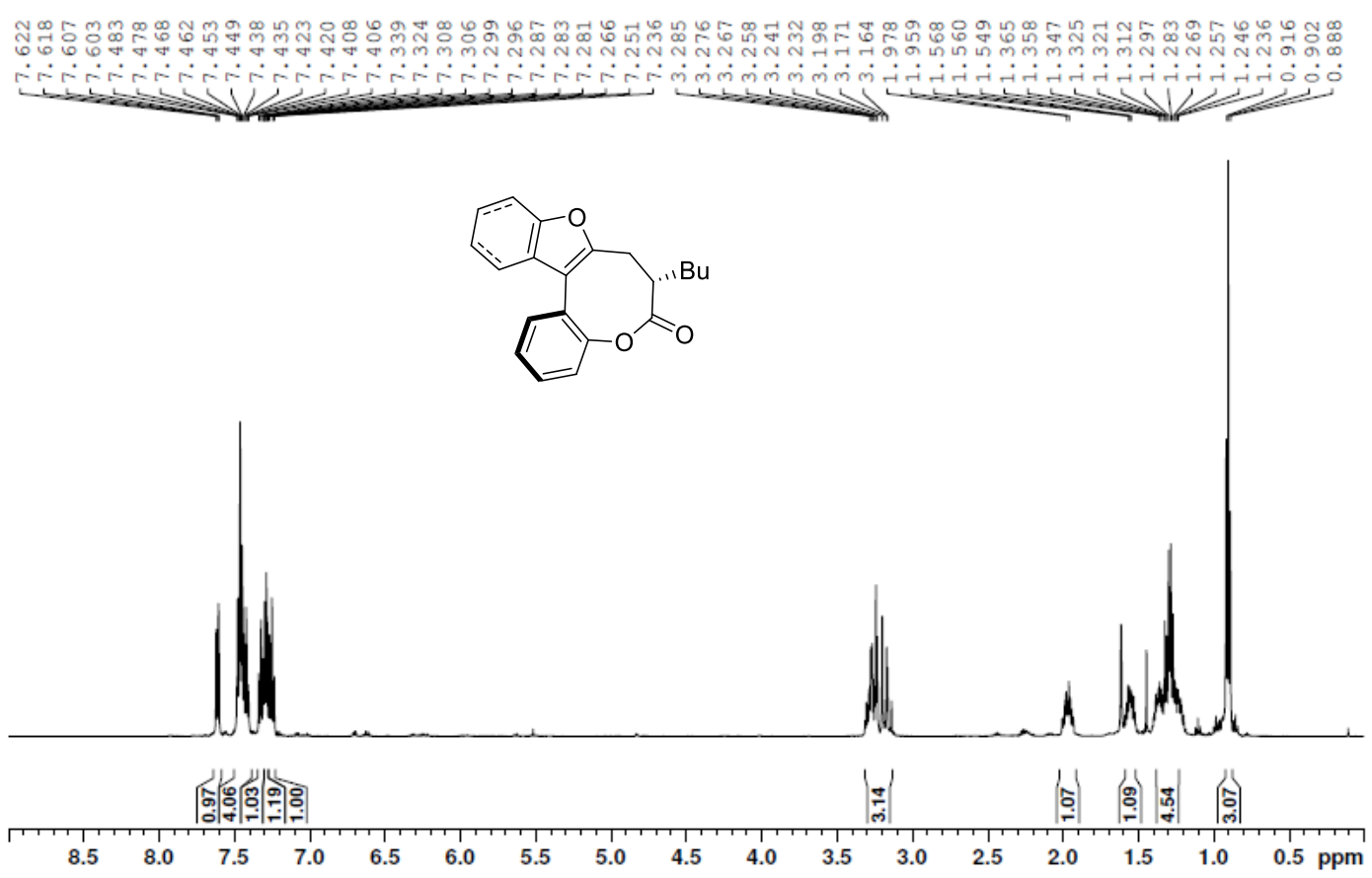

อดบ 24 busy 1 it ring
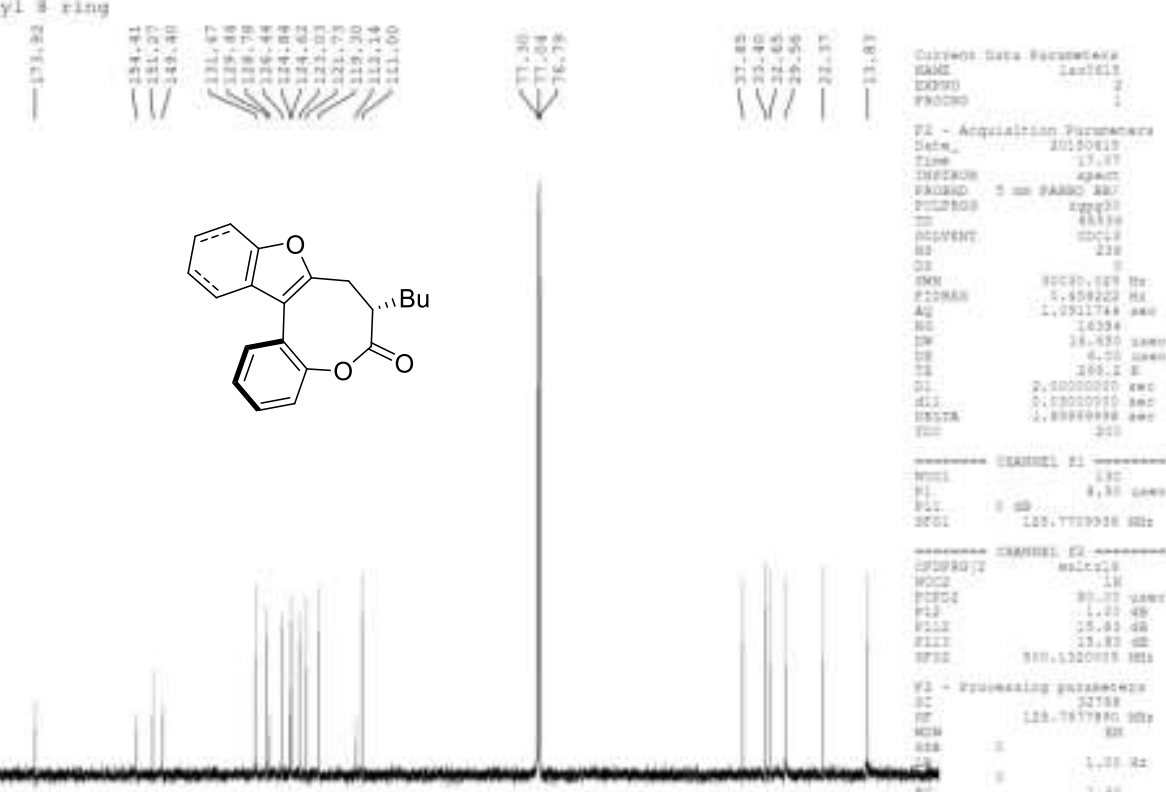

$\begin{array}{llllllllllllllllllllllllllll}190 & 180 & 170 & 160 & 150 & 140 & 130 & 120 & 110 & 100 & 90 & 80 & 70 & 60 & 50 & 40 & 30 & 20 & \text { ppm }\end{array}$ 
(M, S)-7-pentyl-7,8-dihydro-6H-benzo[2,3]oxocino[5,4-b]benzofuran-6-one. (Scheme 4a, 4d)
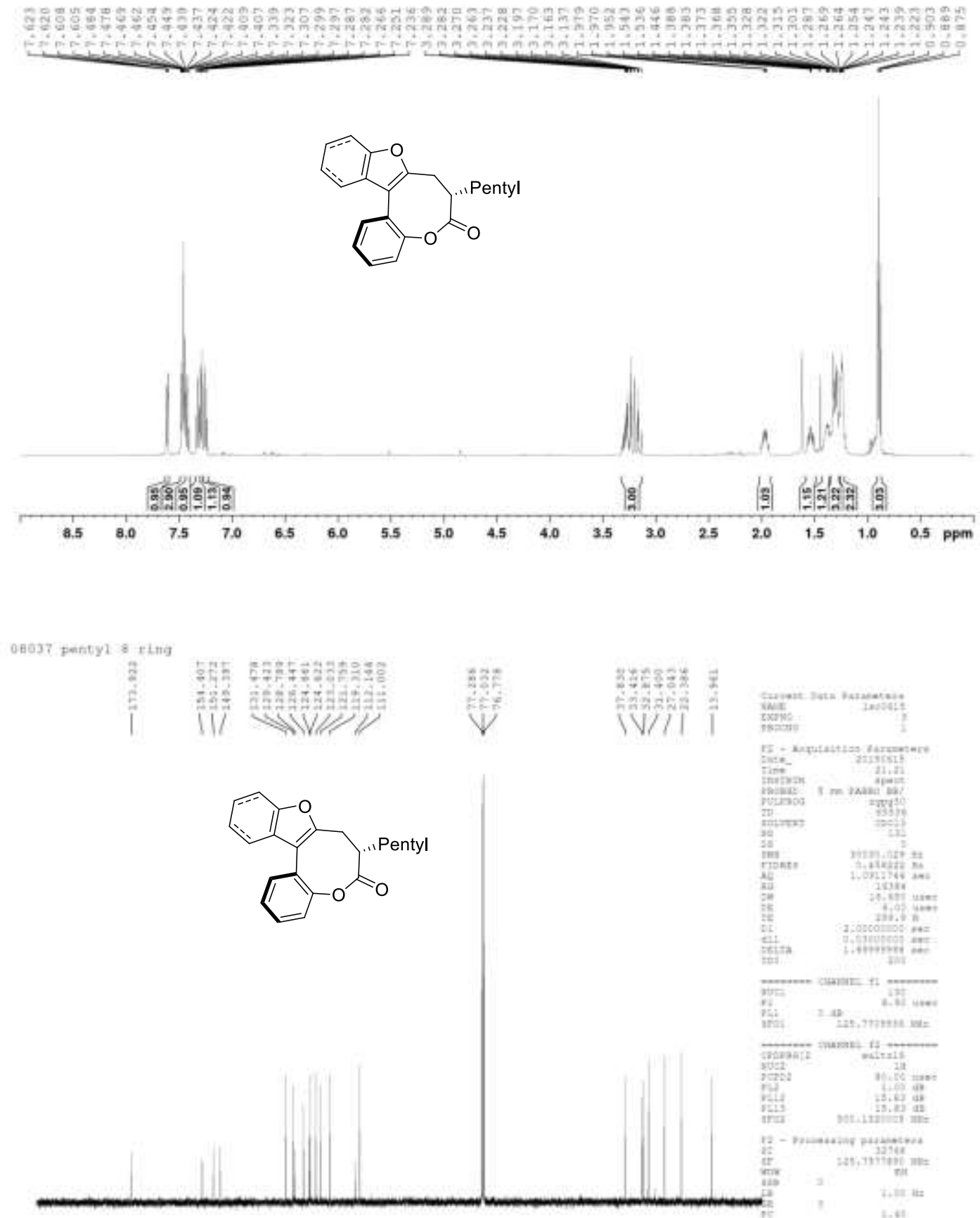

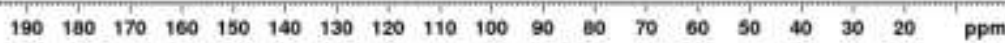


(M, S)-7-hexyl-7,8-dihydro-6H-benzo[2,3]oxocino[5,4-b]benzofuran-6-one. (Scheme 4a, 4e)

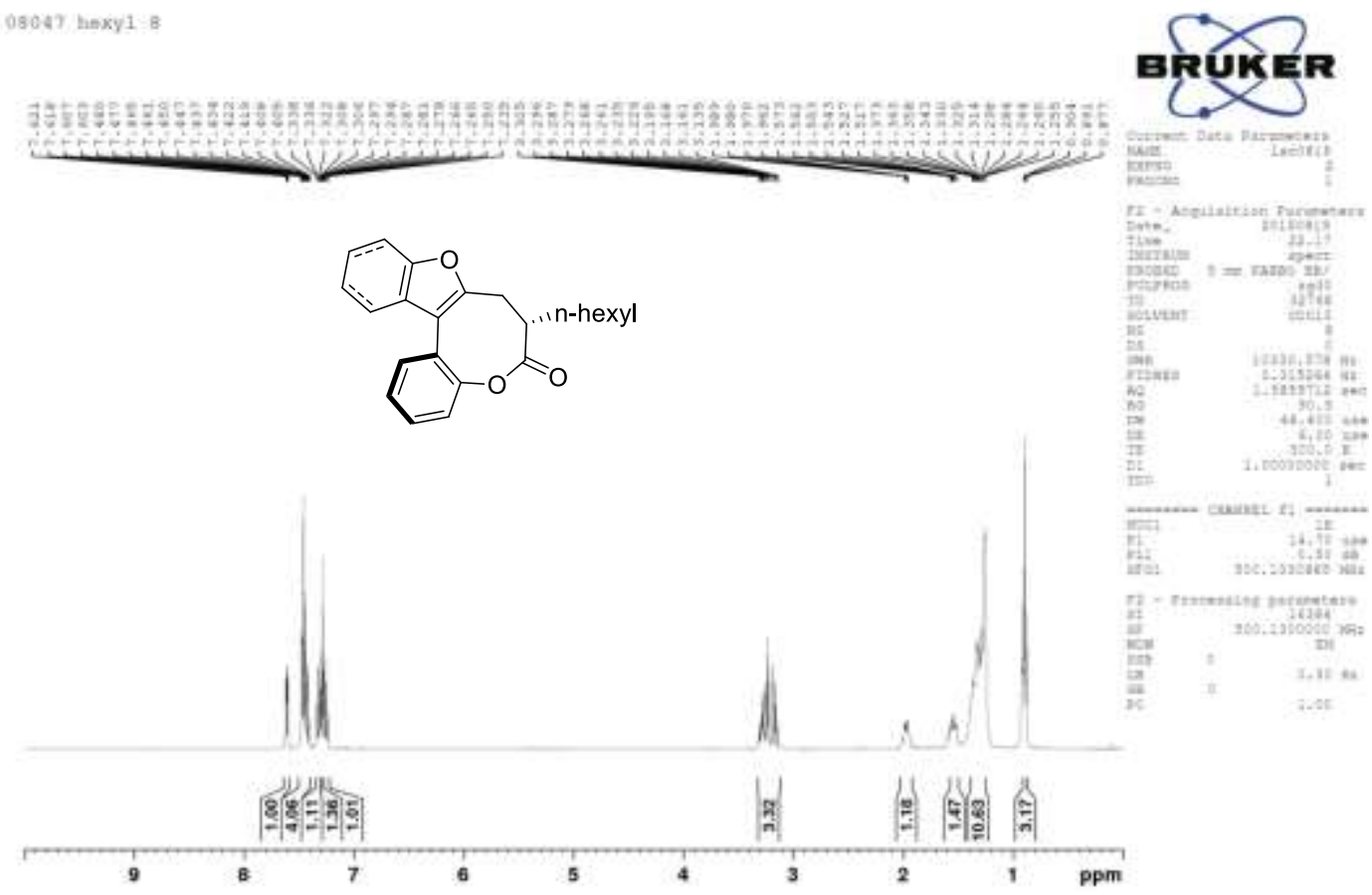

08002 hexyl 8

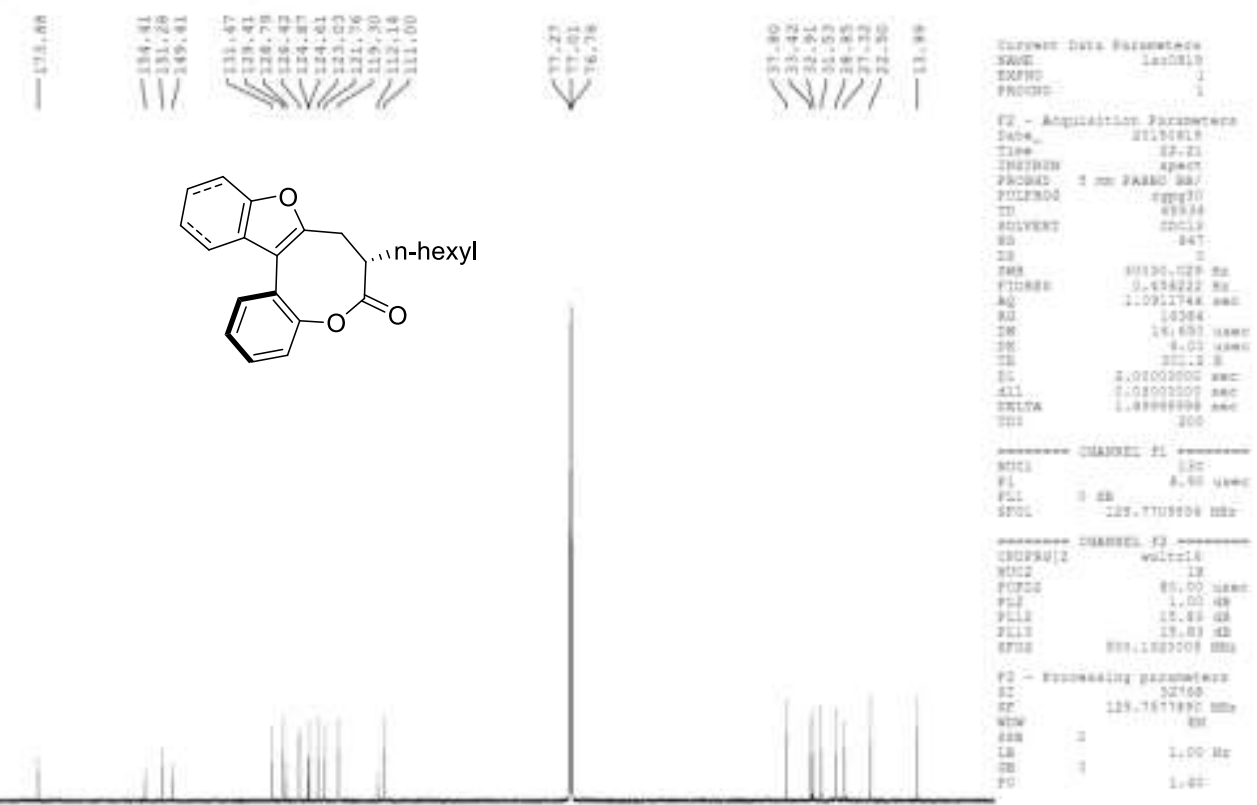

$\begin{array}{lllllllllllllllllllllllllll}190 & 180 & 170 & 160 & 150 & 140 & 130 & 120 & 110 & 100 & 90 & 80 & 70 & 60 & 50 & 40 & 30 & 20 & \mathrm{ppm}\end{array}$ 
(M, S)-7-benzyl-7,8-dihydro-6H-benzo[2,3] oxocino[5,4-b]benzofuran-6-one. (Scheme 4a, 4f)
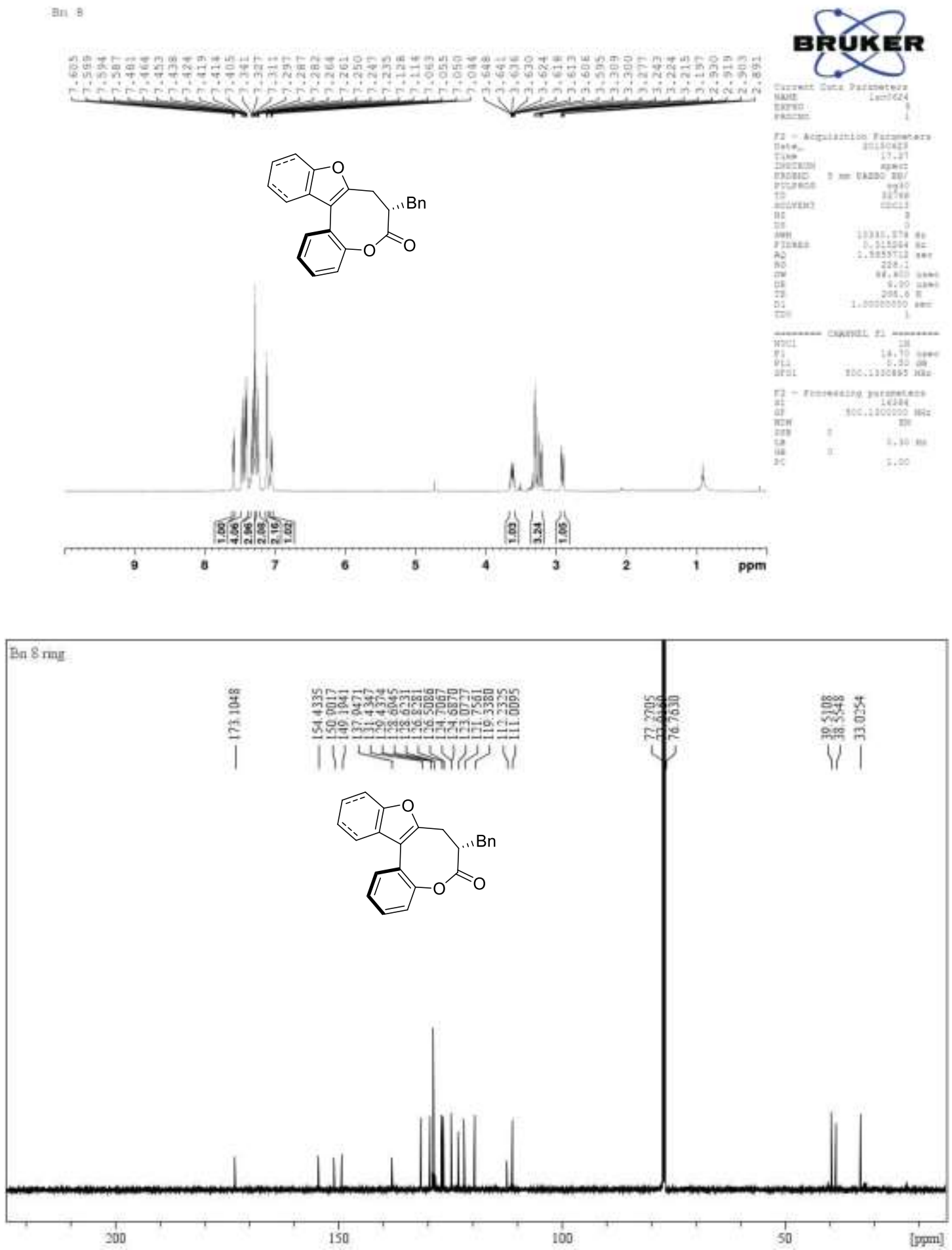
(M, S)-7-(3-((tert-butyldimethylsilyl)oxy)propyl)-7,8-dihydro-6H-benzo[2,3]oxocino[5,4-b] benzofuran-6-one. (Scheme 4a, 4g)

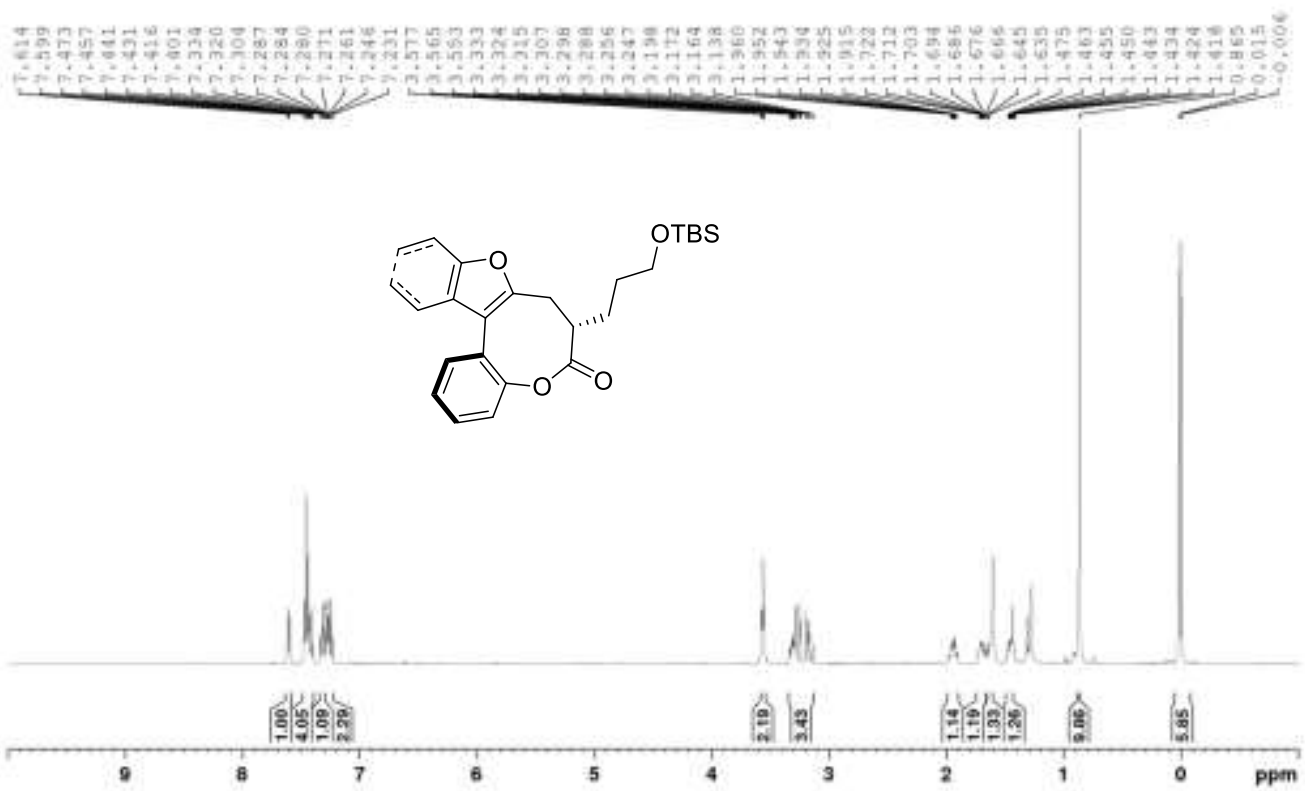

08038 TBSOCHZCH?

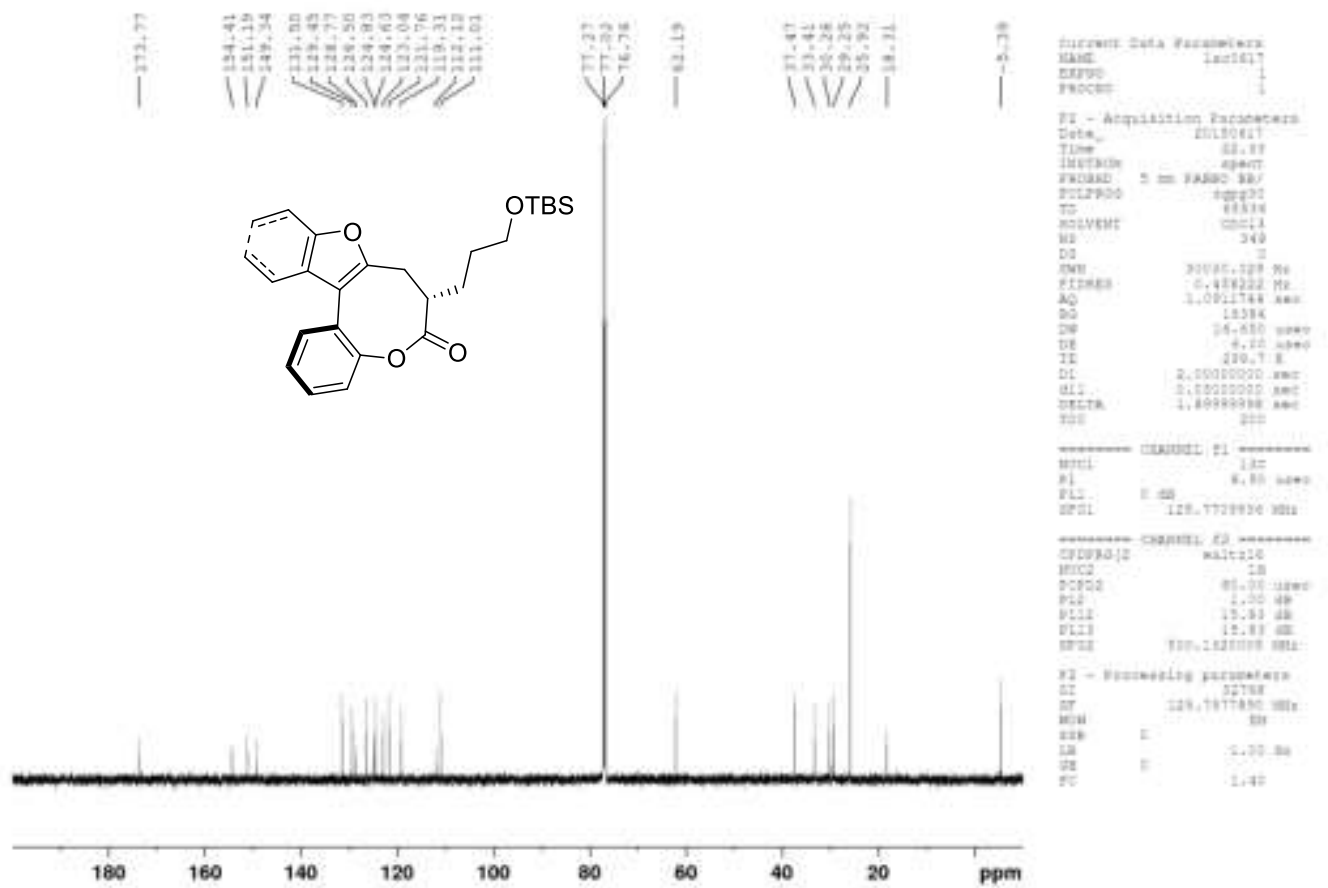


$(M, S)$-7-butyl-3,11-dimethyl-7,8-dihydro-6H-benzo[2,3]oxocino[5,4- $b$ ]benzofuran-6-one. (Scheme 4a, 4h)
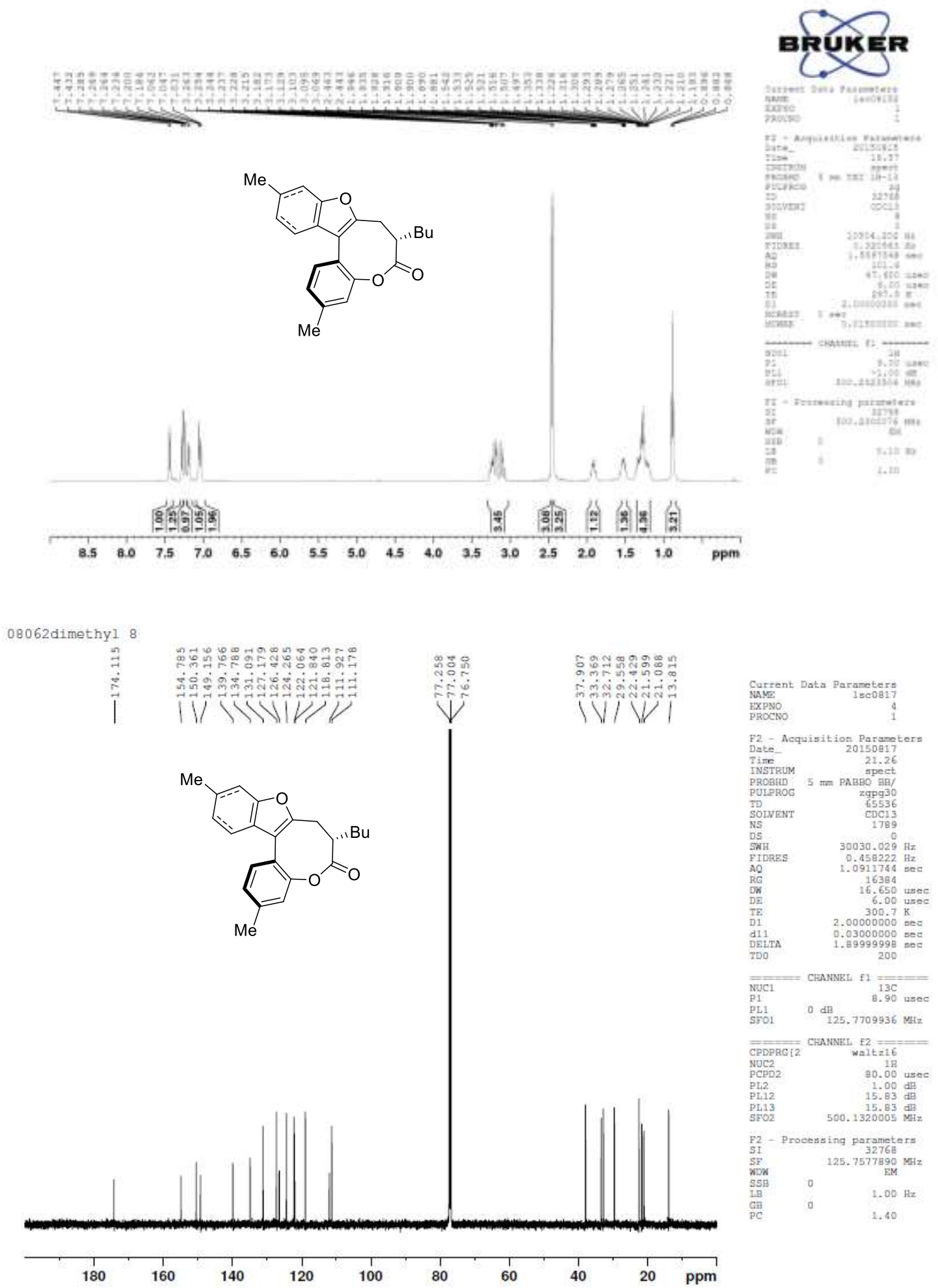
$(M, S)$-3,11-di-tert-butyl-7-butyl-7,8-dihydro-6H-benzo[2,3]oxocino[5,4-b]benzofuran-6-one. (Scheme 4a, 4i)

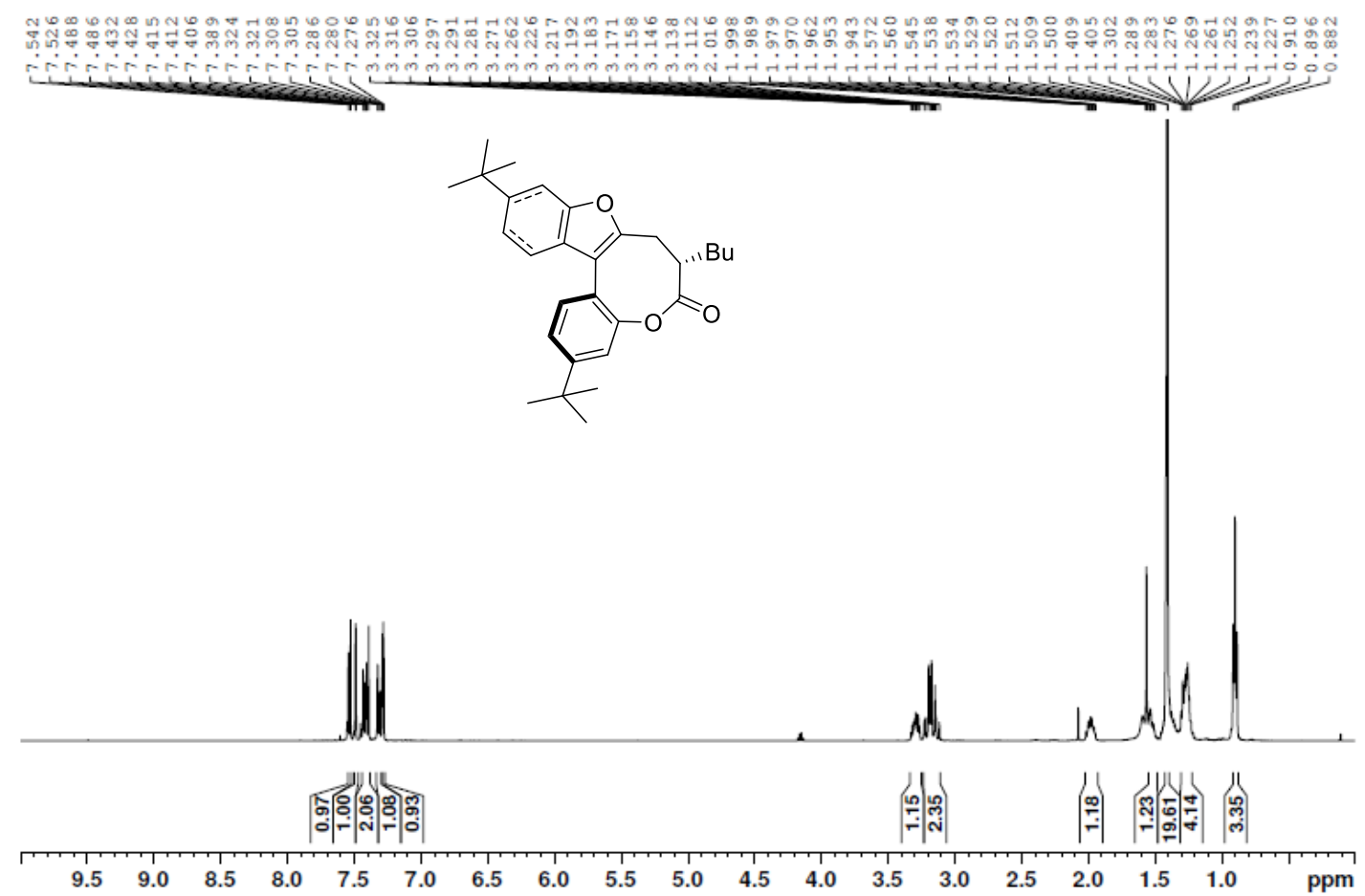
08126 ditertbaty1 $=$
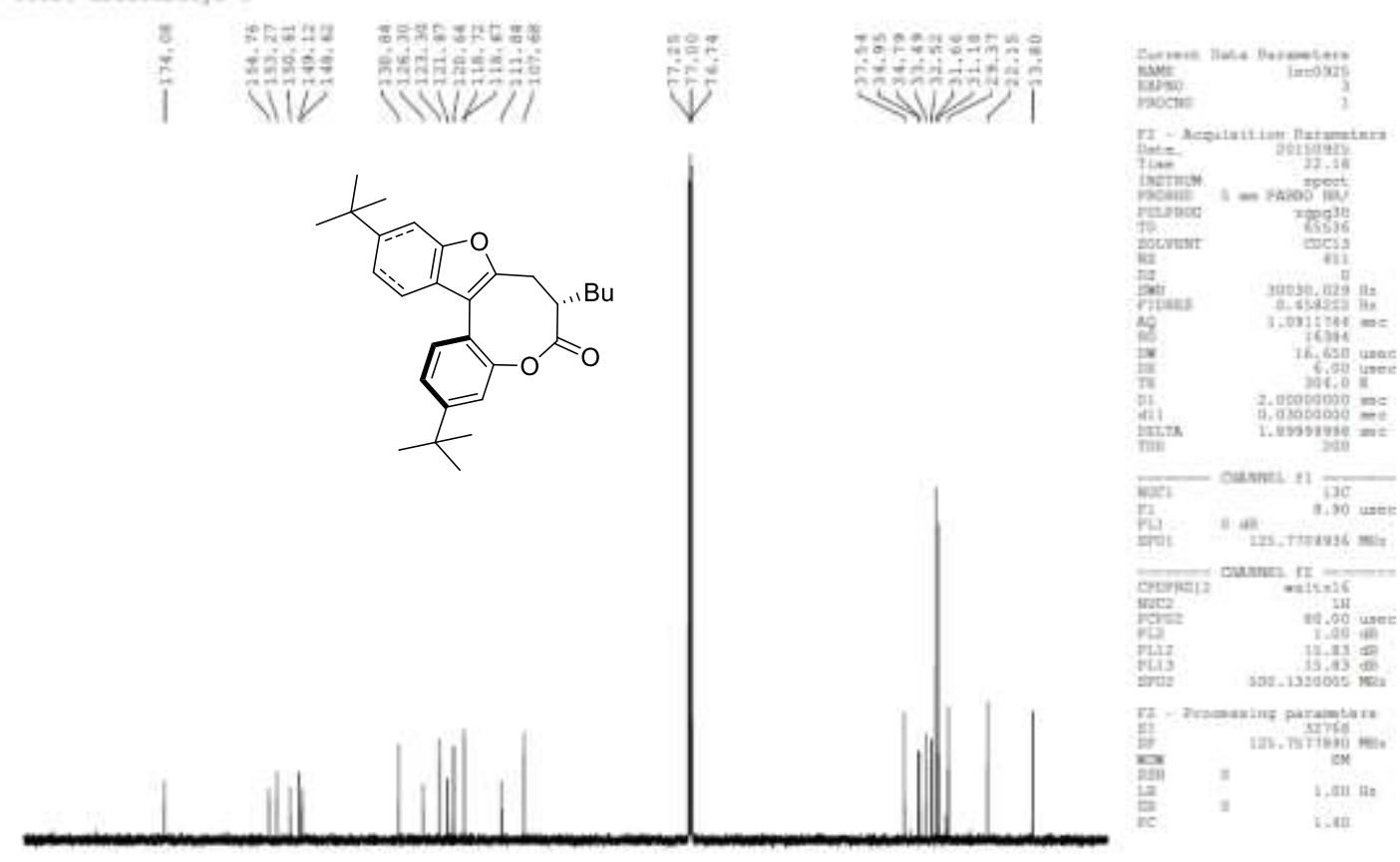

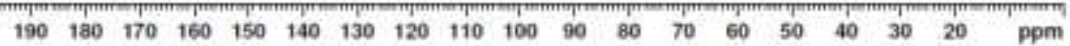


( $M, S$ )-7-butyl-3,11-dimethoxy-7,8-dihydro-6H-benzo[2,3] oxocino[5,4-b]benzofuran-6-one. (Scheme 4a, 4j)
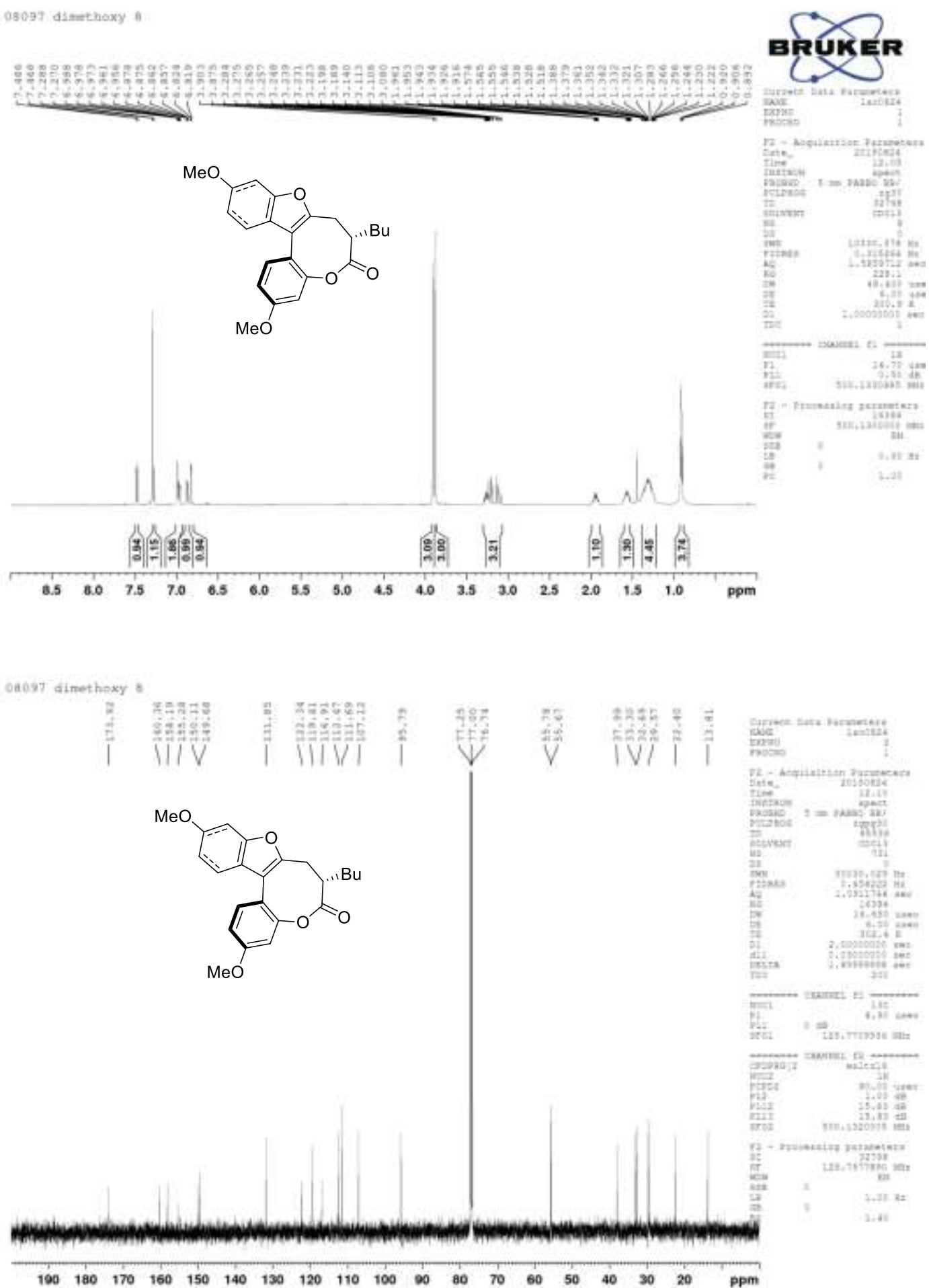
( $M, S$ )-7-butyl-3,11-difluoro-7,8-dihydro-6H-benzo[2,3] oxocino[5,4-b]benzofuran-6-one. (Scheme 4a, 4k)
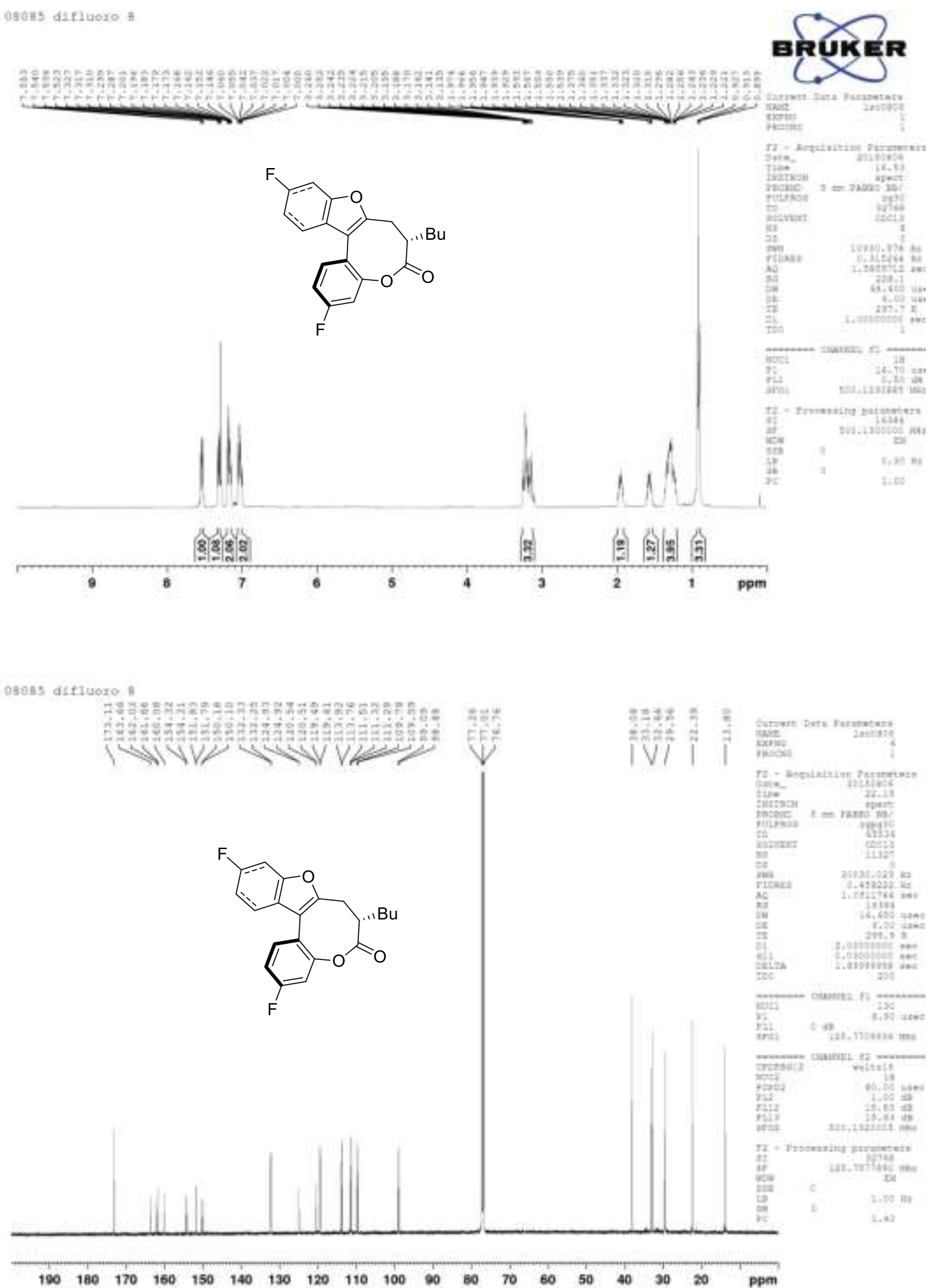
$(M, S)$-7-butyl-3,11-dichloro-7,8-dihydro-6H-benzo[2,3] oxocino[5,4-b]benzofuran-6-one. (Scheme 4a, 4l)
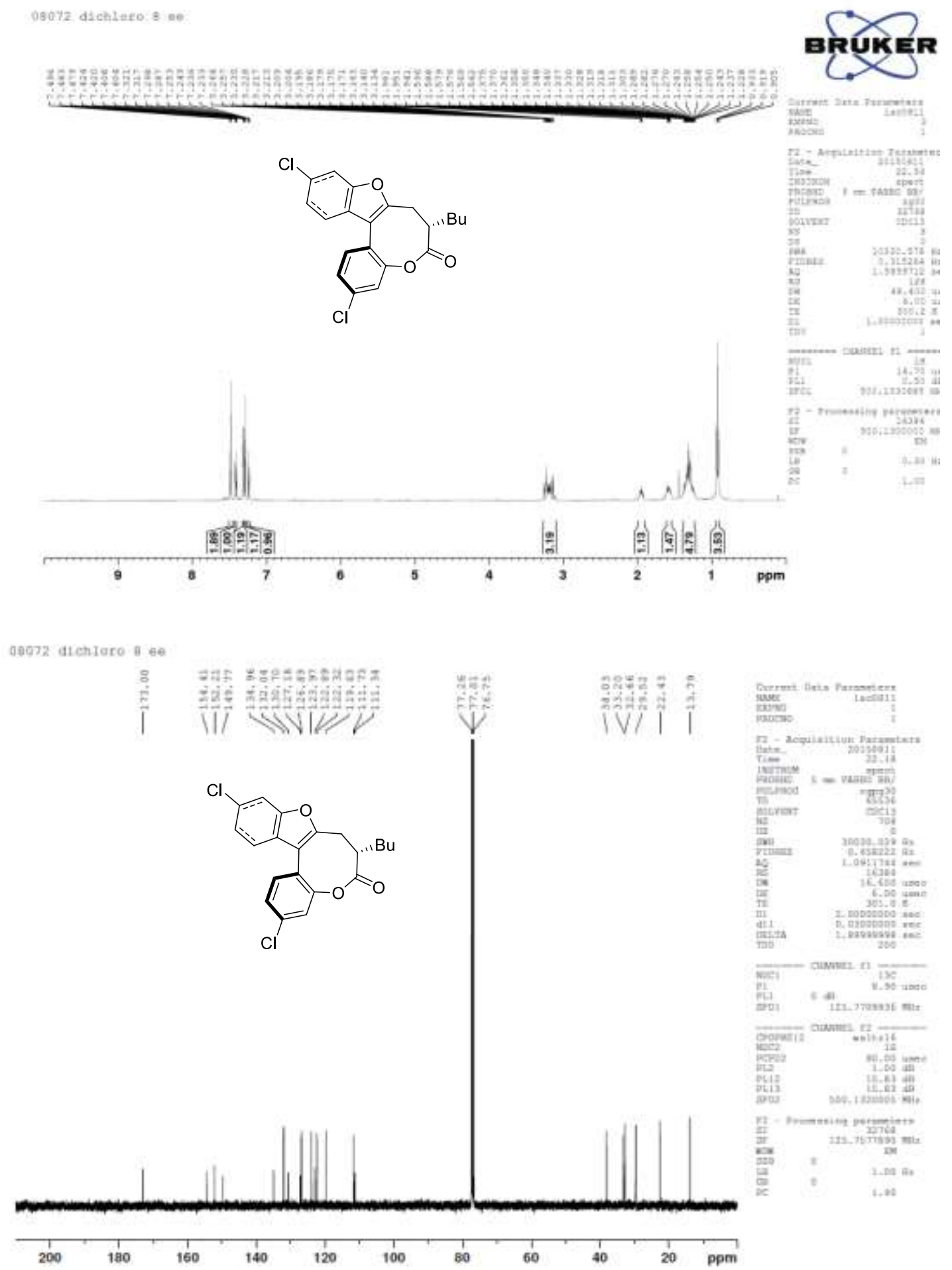
( $M, S)$-3,11-dibromo-7-butyl-7,8-dihydro-6H-benzo[2,3] oxocino[5,4- $b$ ]benzofuran-6-one. (Scheme 4a, 4m)
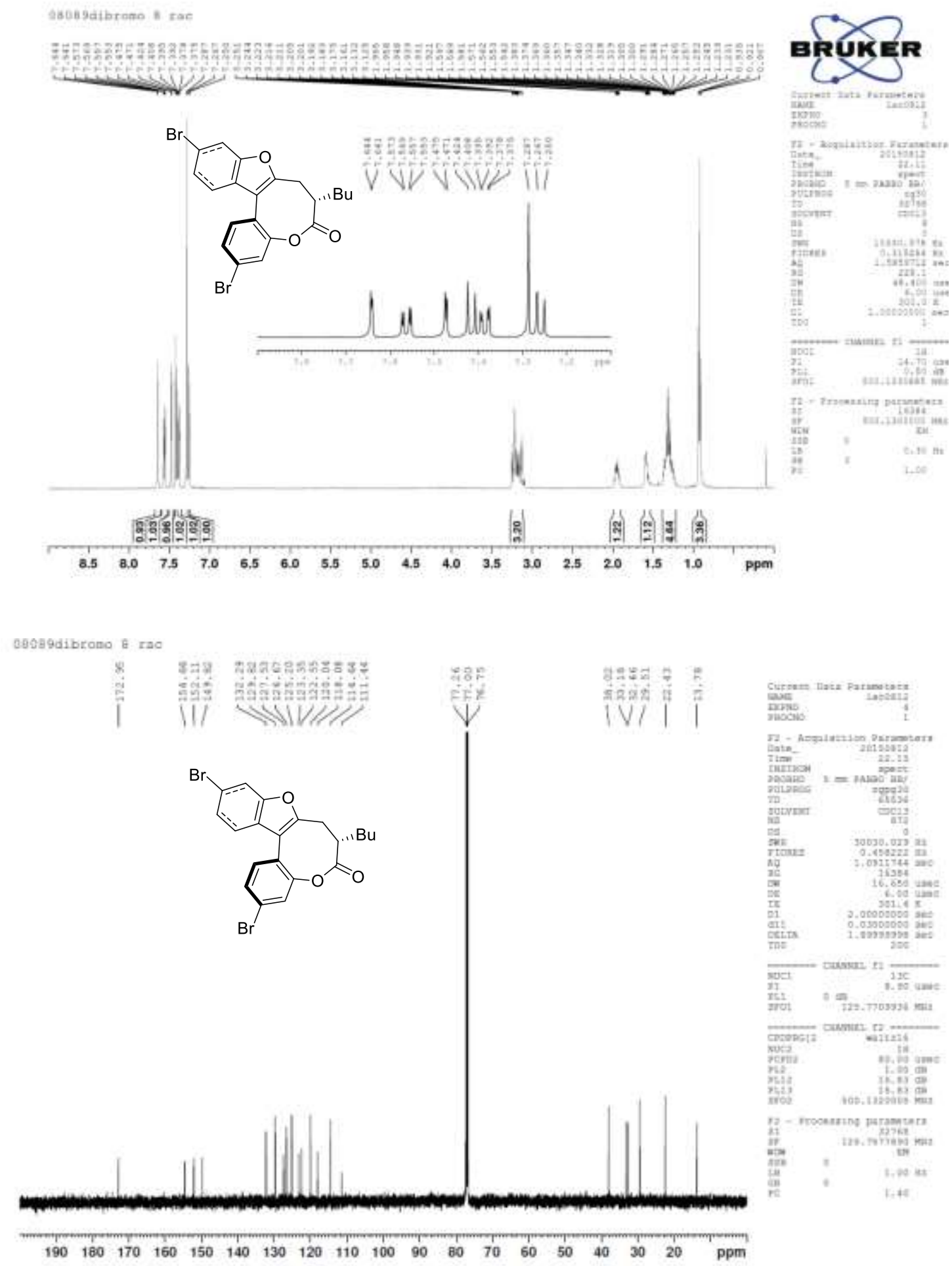
$(M, S)$-7-butyl-2,12-dimethyl-7,8-dihydro-6H-benzo[2,3] oxocino[5,4- $b$ ]benzofuran-6-one. (Scheme 4a, 4n)

08109 53dinechil :

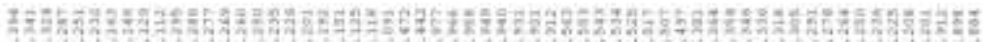
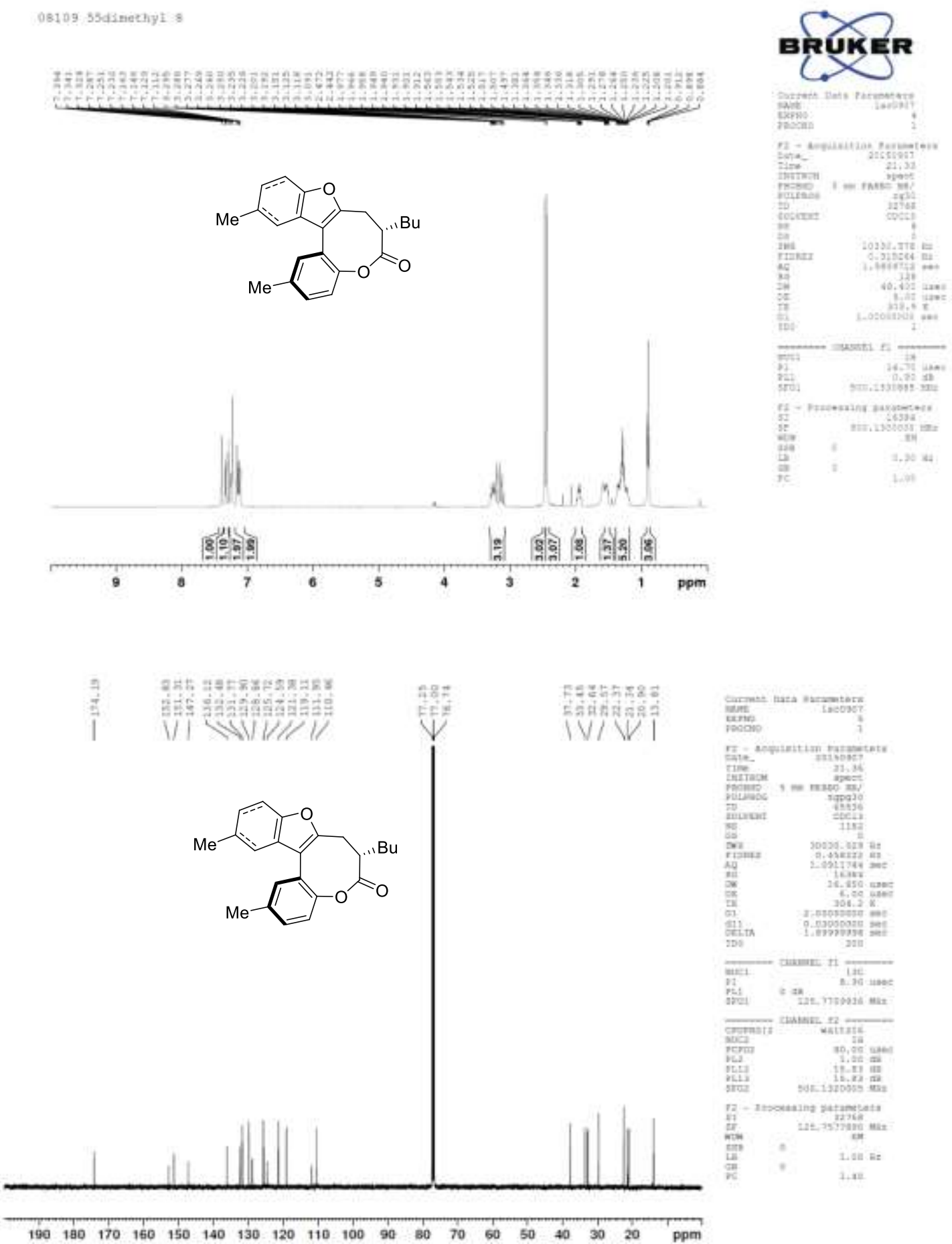
$(M, S)$-2,12-di-tert-butyl-7-butyl-7,8-dihydro-6H-benzo[2,3] oxocino[5,4-b]benzofuran-6-one. (Scheme 4a, 4o)
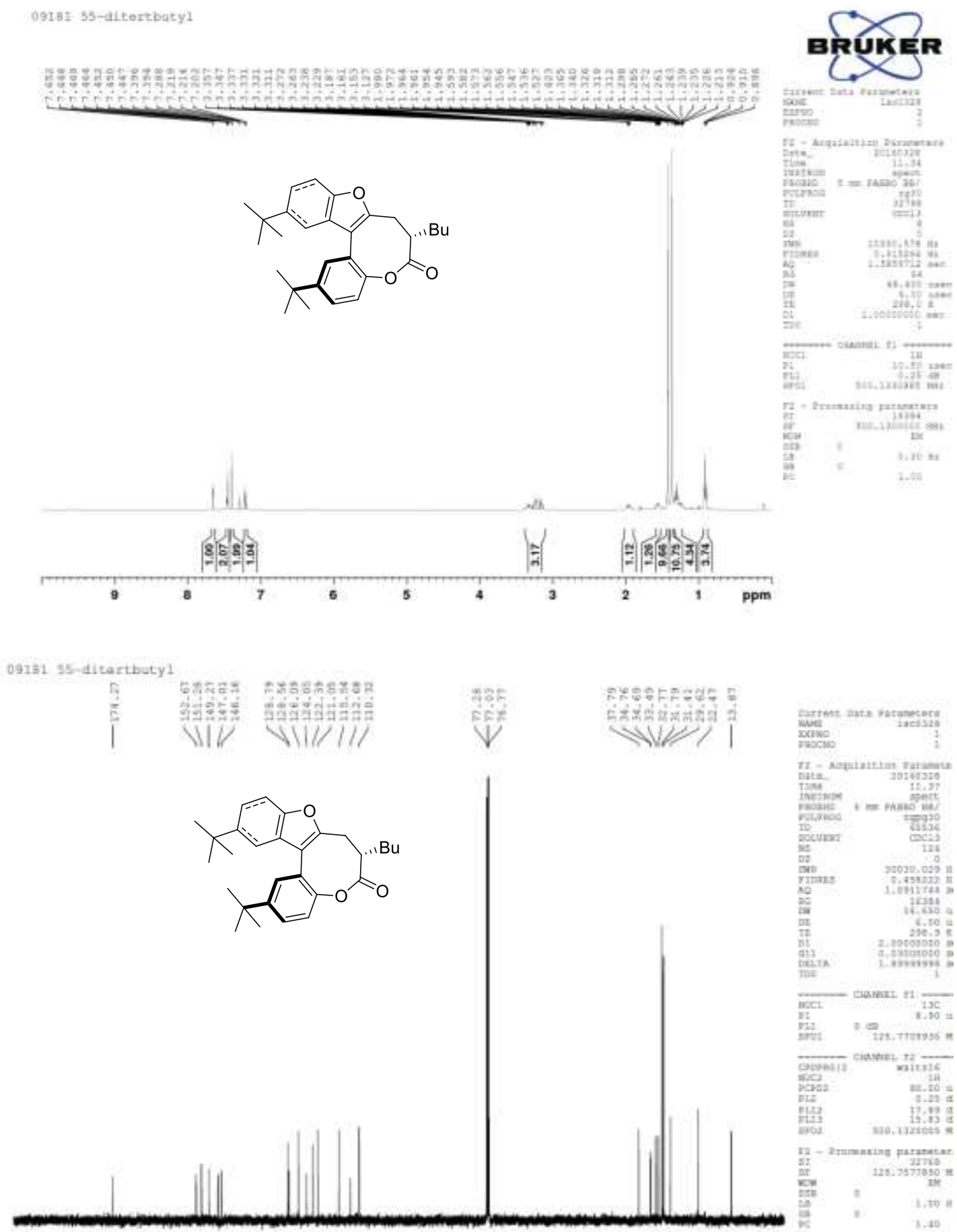

$\begin{array}{llllllllllllllllll}190 & 180 & 170 & 160 & 150 & 140 & 130 & 120 & 110 & 100 & 90 & 80 & 70 & 60 & 50 & 40 & 30 & 20\end{array}$ 
( $M, S)$-2,12-dibromo-7-butyl-7,8-dihydro-6H-benzo[2,3] oxocino[5,4-b]benzofuran-6-one. (Scheme 4a, 4p)
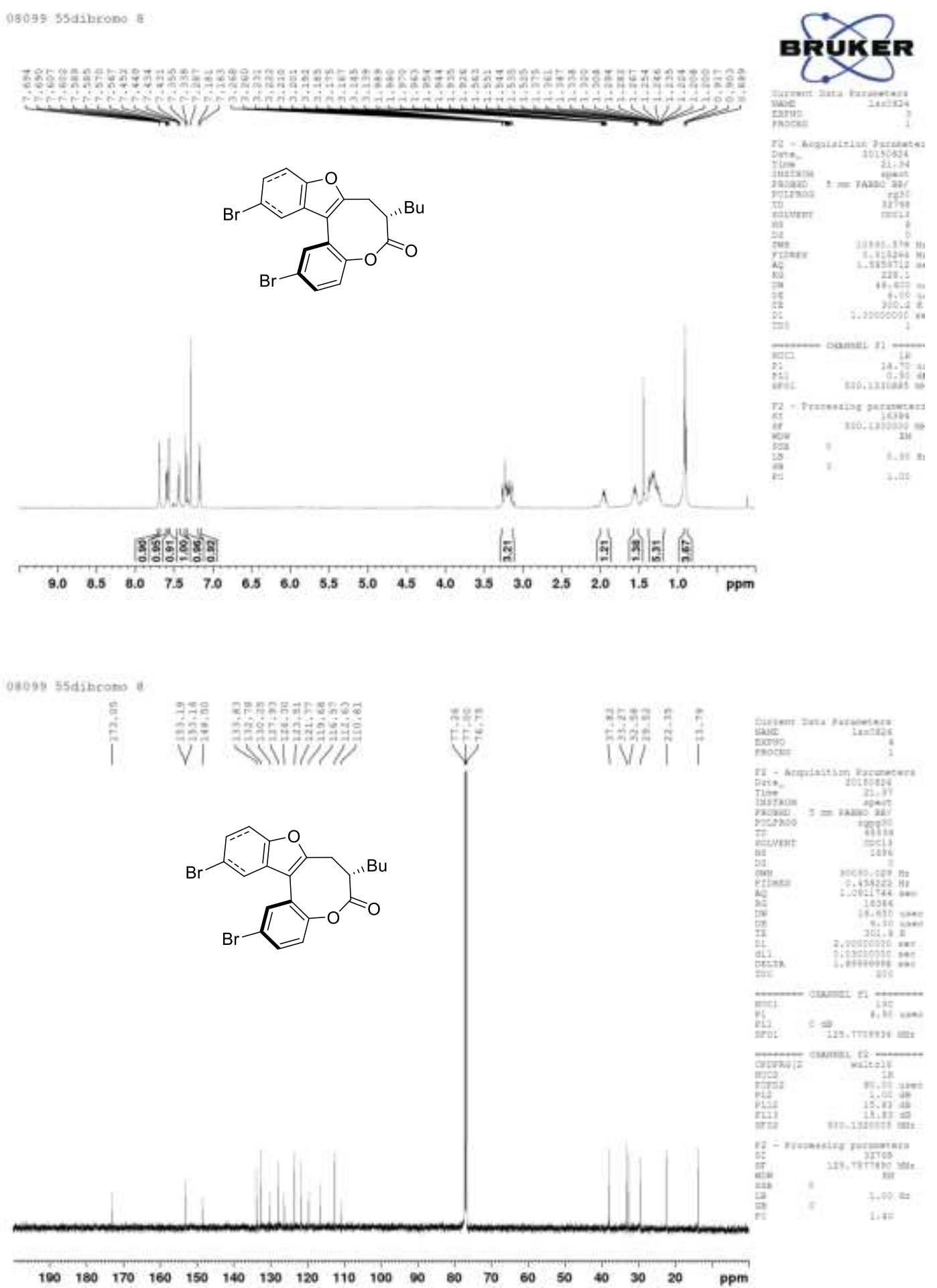
(M, 7S)-7-butyl-8-methyl-7,8-dihydro-6H-benzo[2,3] oxocino[5,4-b]benzofuran-6-one.

(Scheme 4a, 4q)
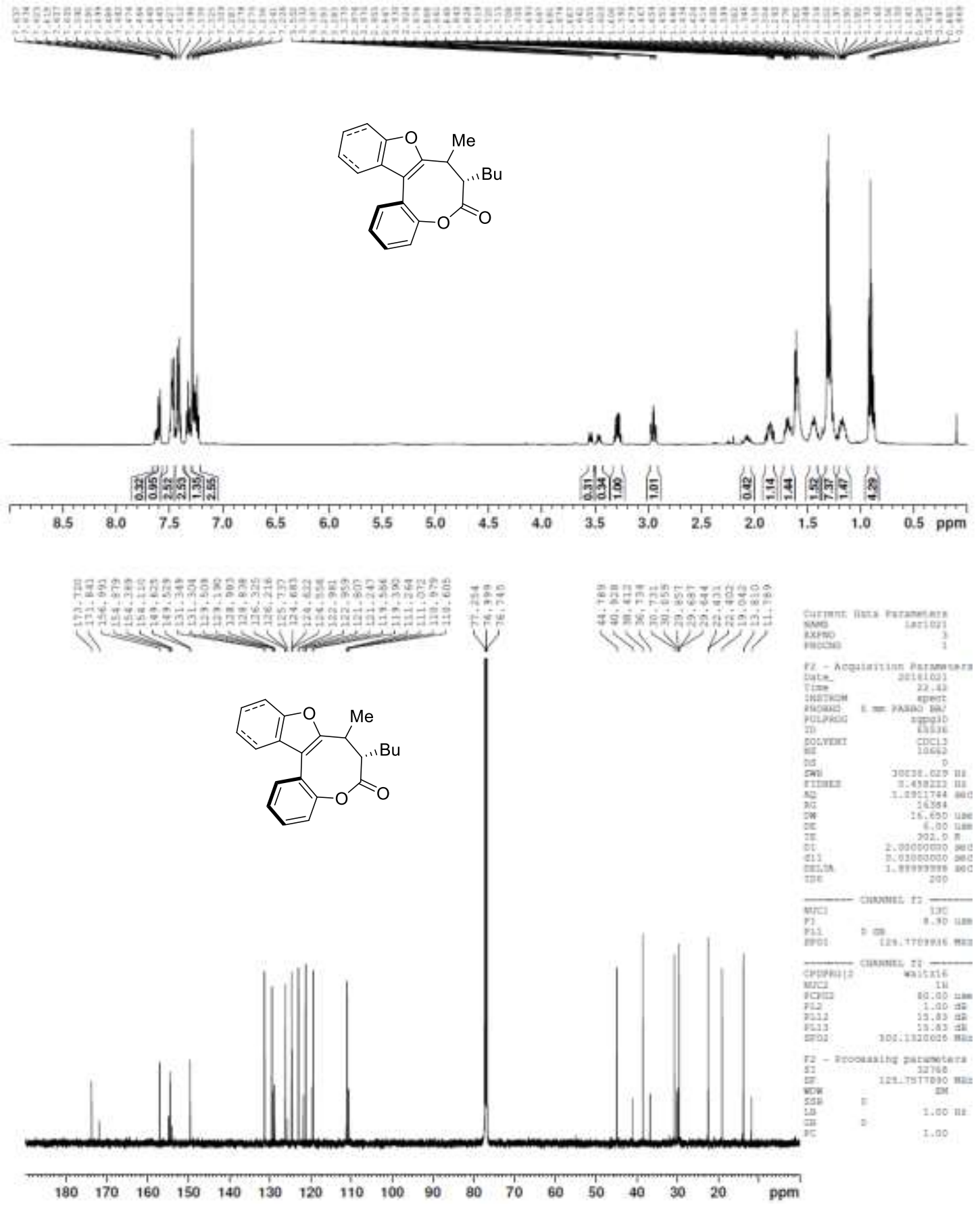
$(M, 7 R, 8 S)$-7-isopropyl-8-methyl-7,8-dihydro-6H-benzo[2,3]oxocino[5,4- $b$ ]benzofuran-6-one. (Scheme 4a, 4r)
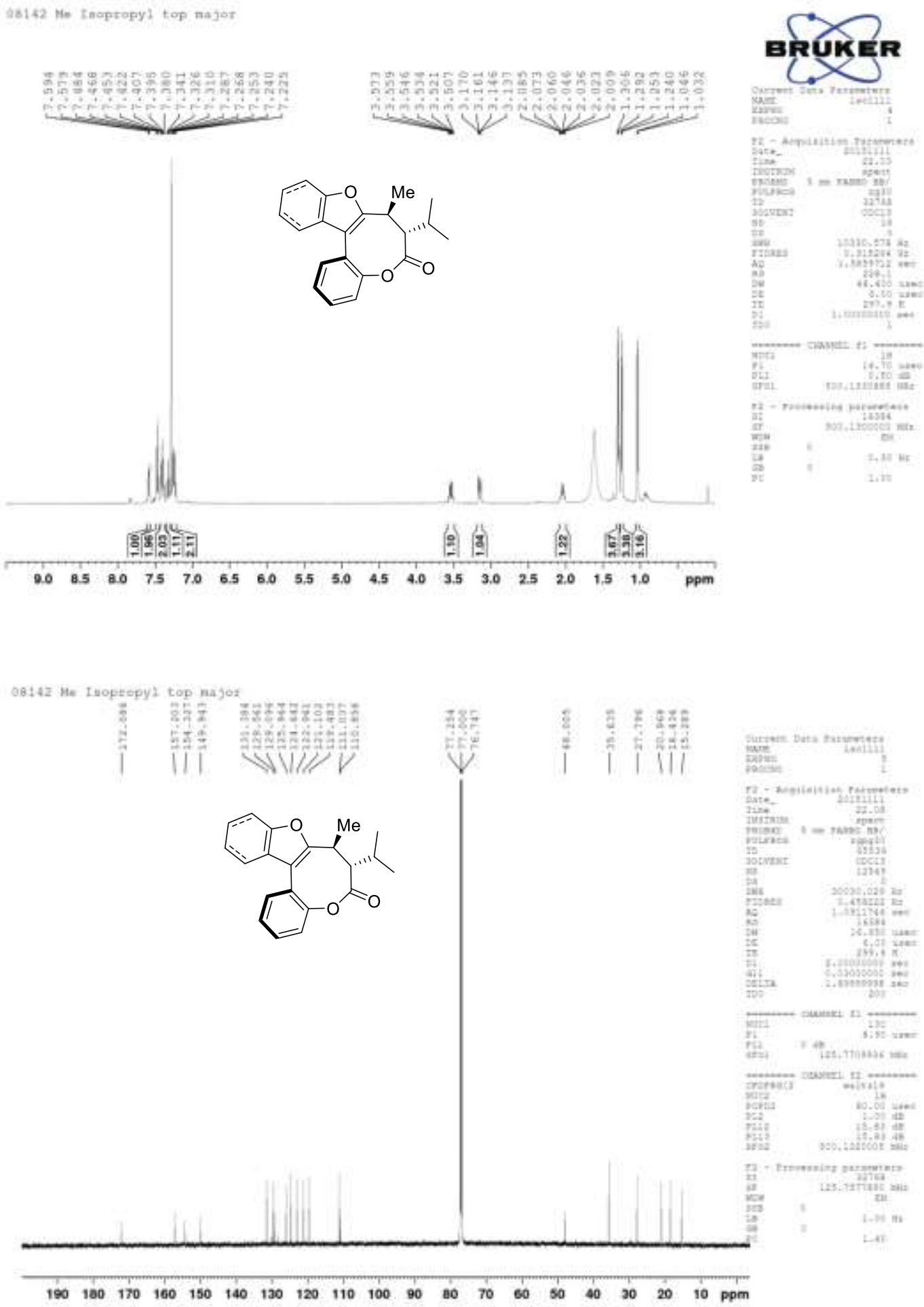
( $M, 7 R, 8 R$ )-7-isopropyl-8-methyl-7,8-dihydro-6H-benzo[2,3] oxocino[5,4- $b$ ]benzofuran-6-one. (Scheme 4a, 4r')
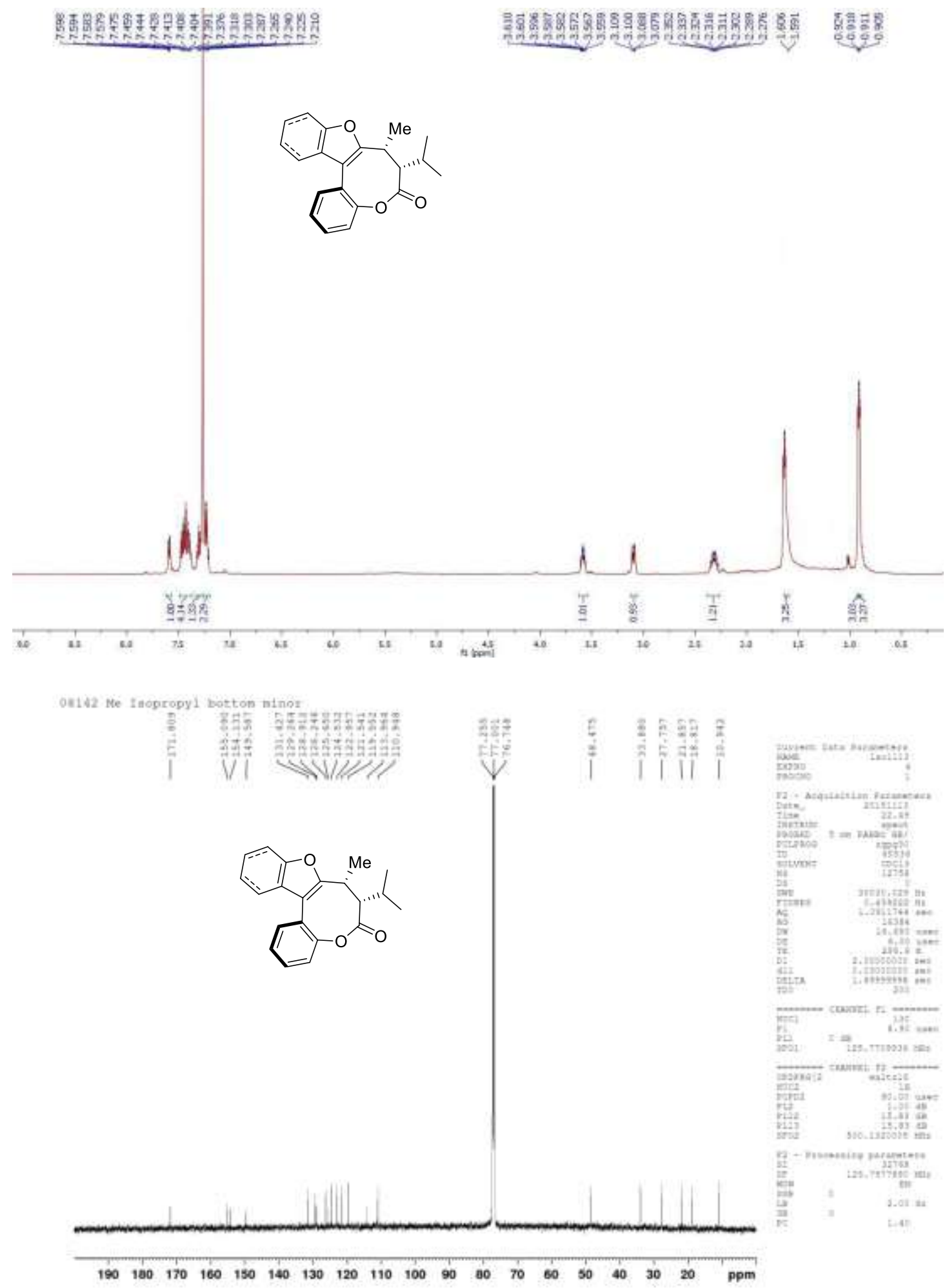
( $M, S$ )-7-butyl-3-methoxy-7,8-dihydro-6H-benzo[2,3] oxocino[5,4-b] benzofuran-6-one.

(Scheme 4b, 4s)

aBo4f noo top najor

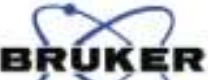

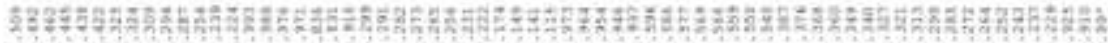
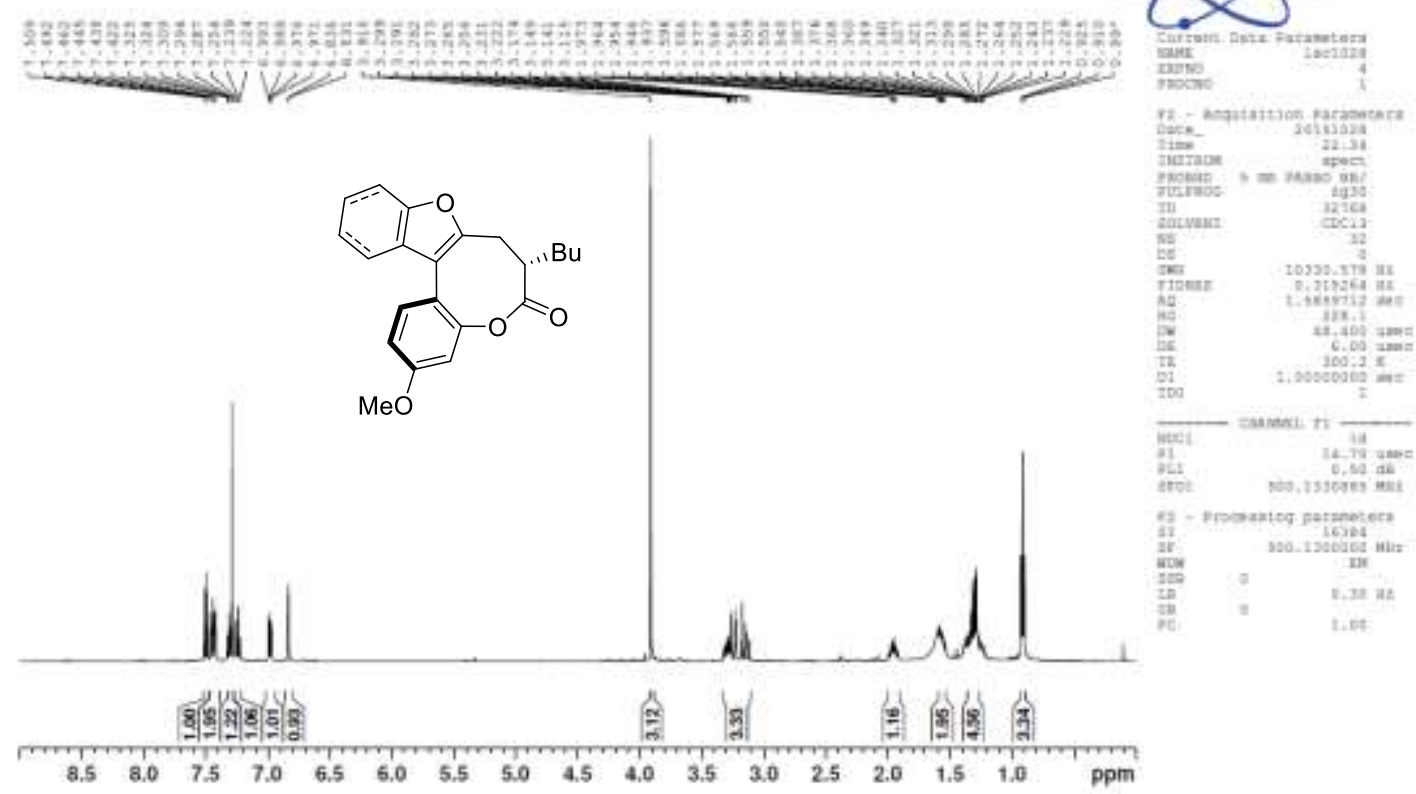

Oebah Mod top major

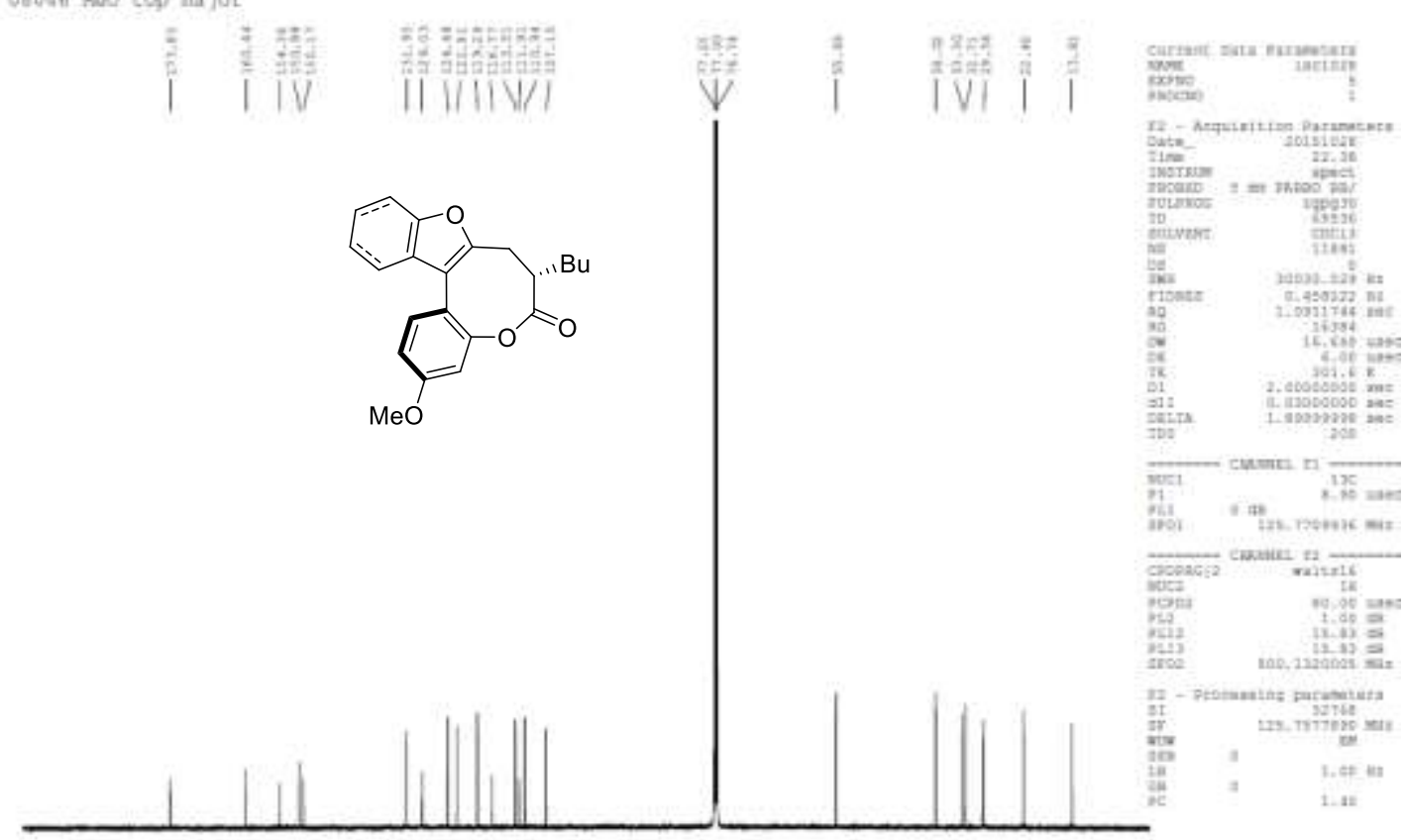

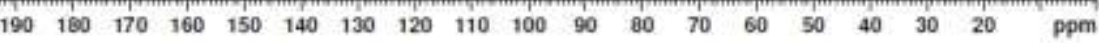


$(M, S)$-7-butyl-12-nitro-7,8-dihydro-6H-benzo[2,3]oxocino[5,4-b]benzofuran-6-one. (Scheme $4 \mathrm{~b}, 4 \mathrm{t})$

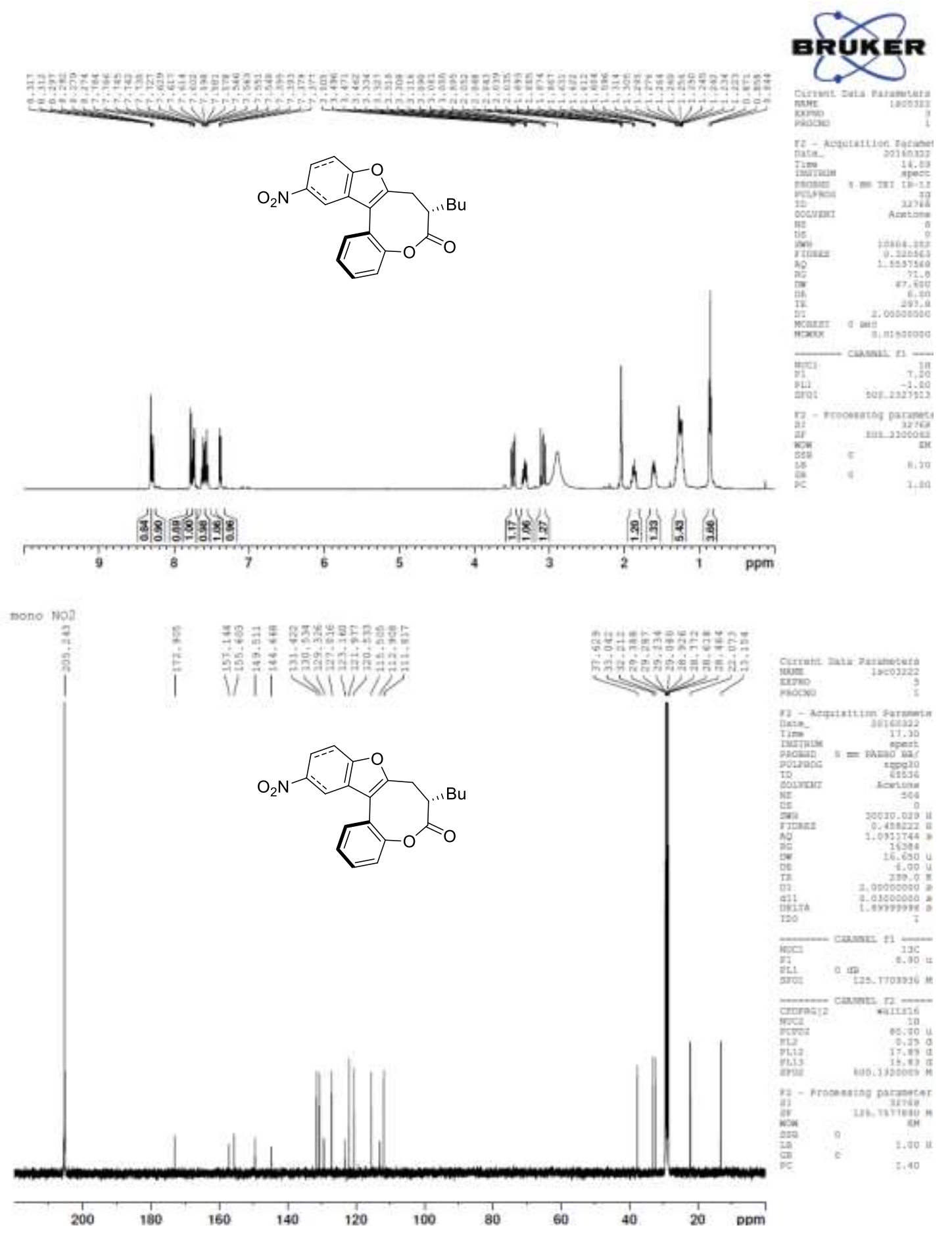


$(M, S)$-7-butyl-9-tosyl-7,8-dihydrobenzo[2,3]oxocino[5,4-b]indol-6(9H)-one. (Scheme 5, 6a)

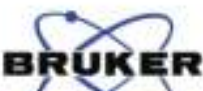

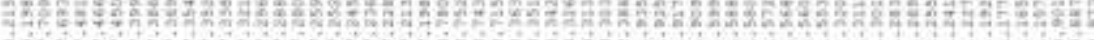
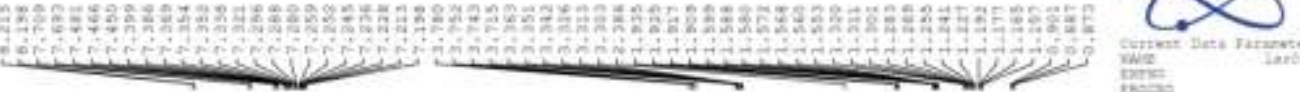

Wide
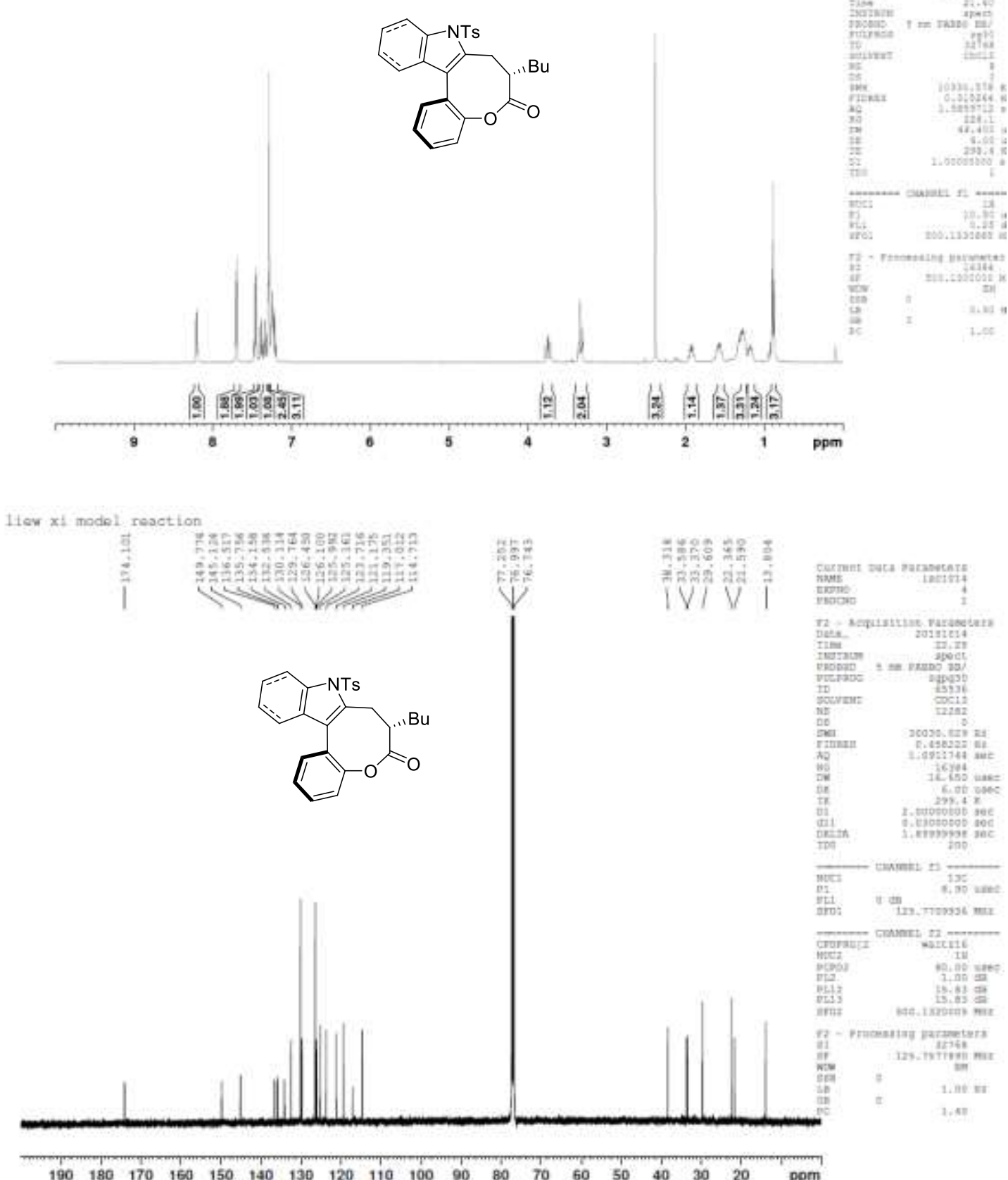
( $M, S$ )-7-butyl-2-methyl-9-tosyl-7,8-dihydrobenzo[2,3] oxocino[5,4-b]indol-6(9H)-one. (Scheme 5,, 6b)

as028 3-Ne entar pdt
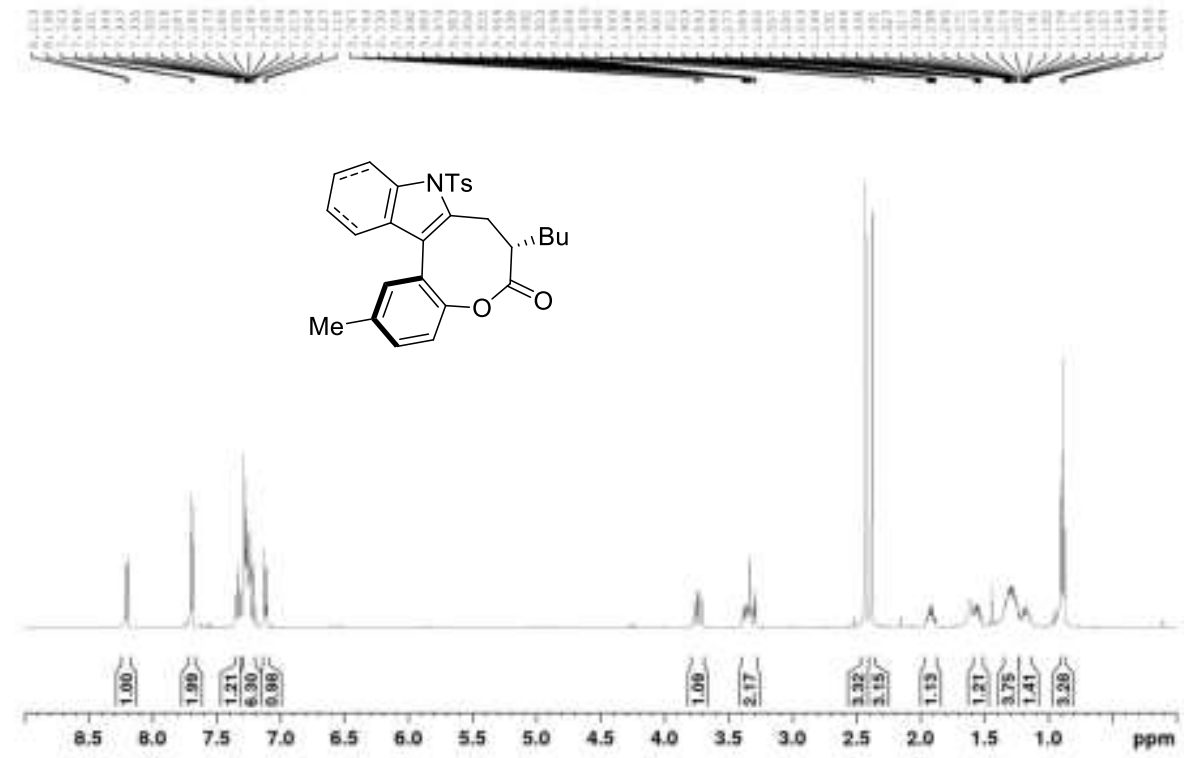

09028 5-Ma estor pdt
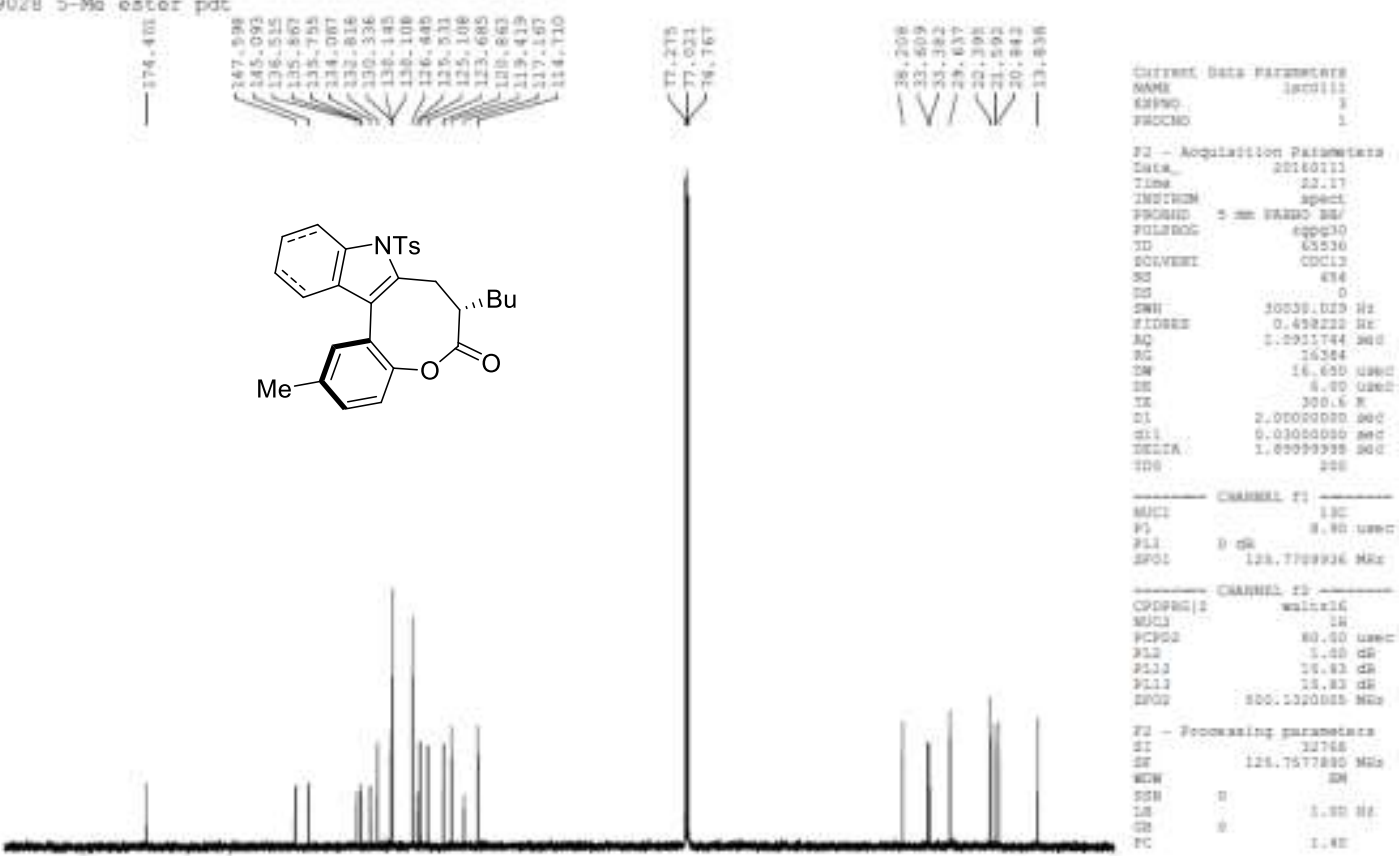

$\begin{array}{lllllllllllllllllll}190 & 180 & 170 & 160 & 150 & 140 & 130 & 120 & 110 & 100 & 90 & 80 & 70 & 60 & 50 & 40 & 30 & 20 & \text { ppm }\end{array}$ 
( $M, S)$-2-(tert-butyl)-7-butyl-9-tosyl-7,8-dihydrobenzo[2,3]oxocino[5,4-b]indol-6(9H)-one. (Scheme 5, 6c)
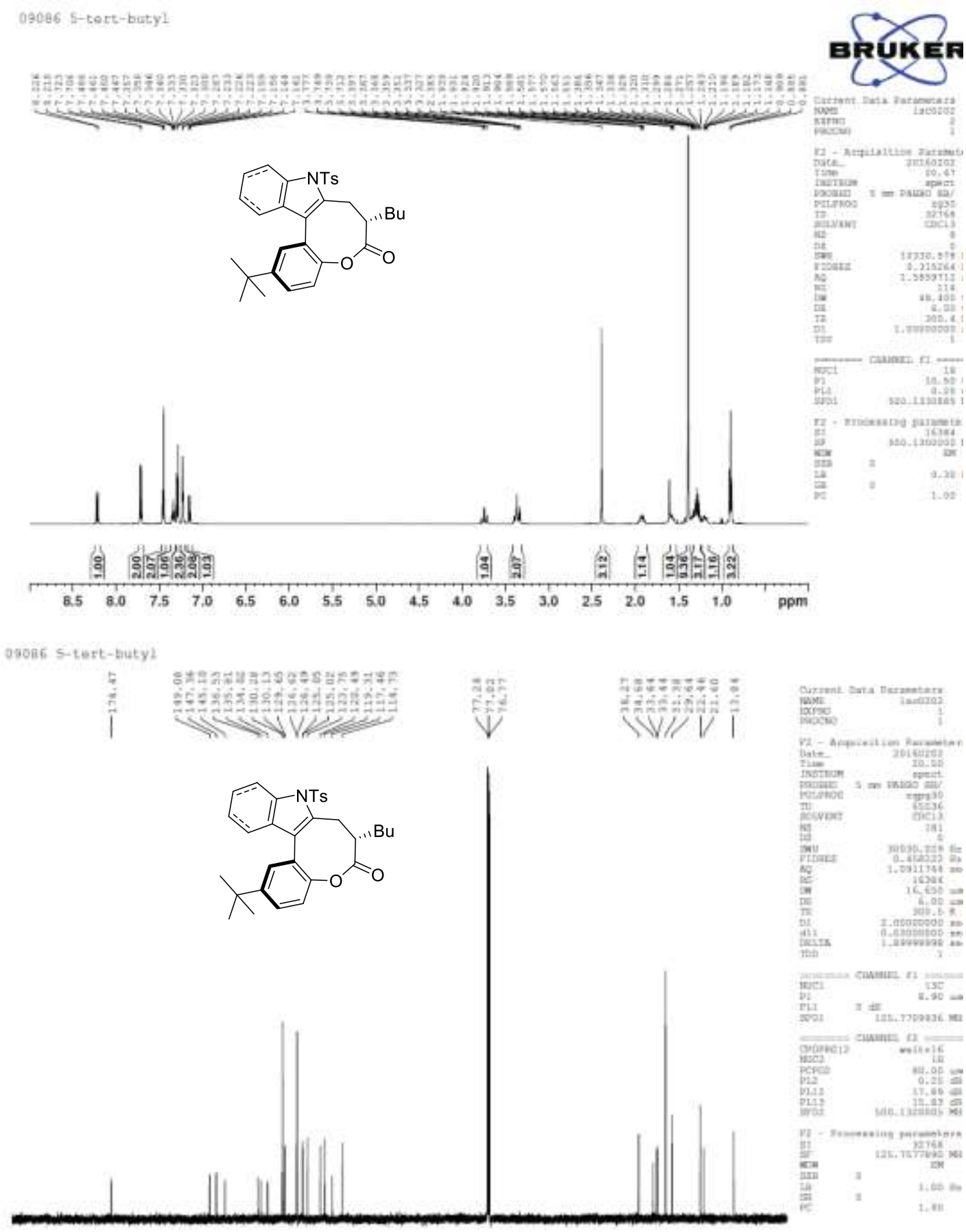

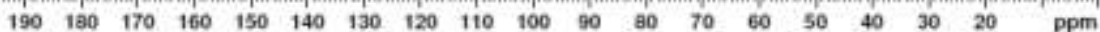


( $M, S$ )-7-butyl-3-methyl-9-tosyl-7,8-dihydrobenzo[2,3] oxocino[5,4-b]indol-6(9H)-one.

(Scheme 5, 6d)

09074 4-Me of pat

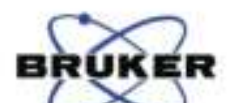

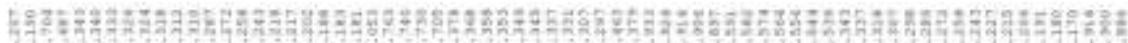
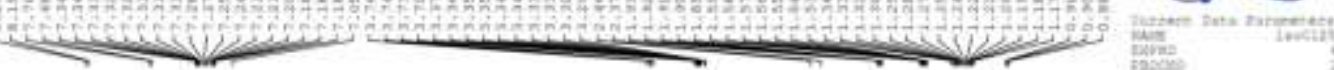<smiles>CCCCC[C@@H]1Cc2c([N+]#[SH])cccc2-c2ccc(C)cc2OC1=O</smiles>

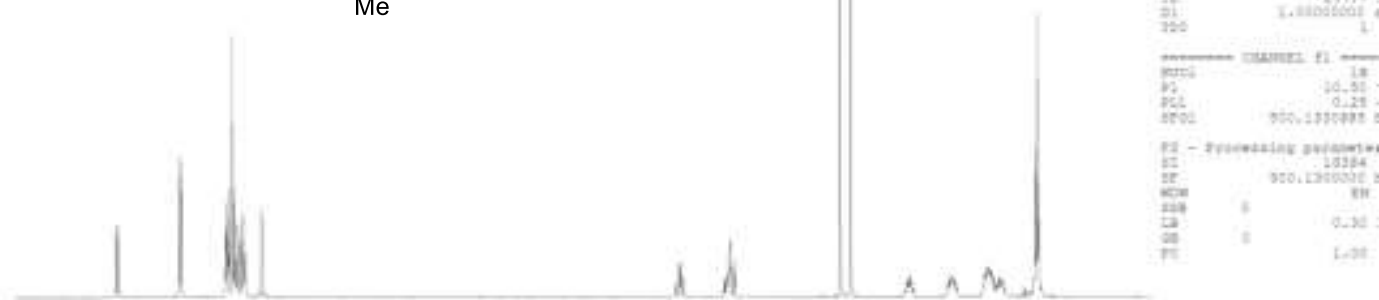

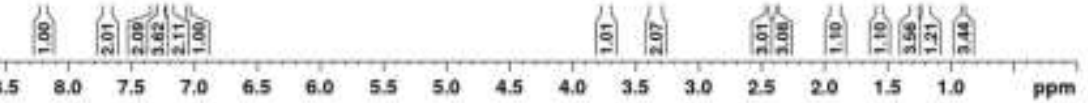
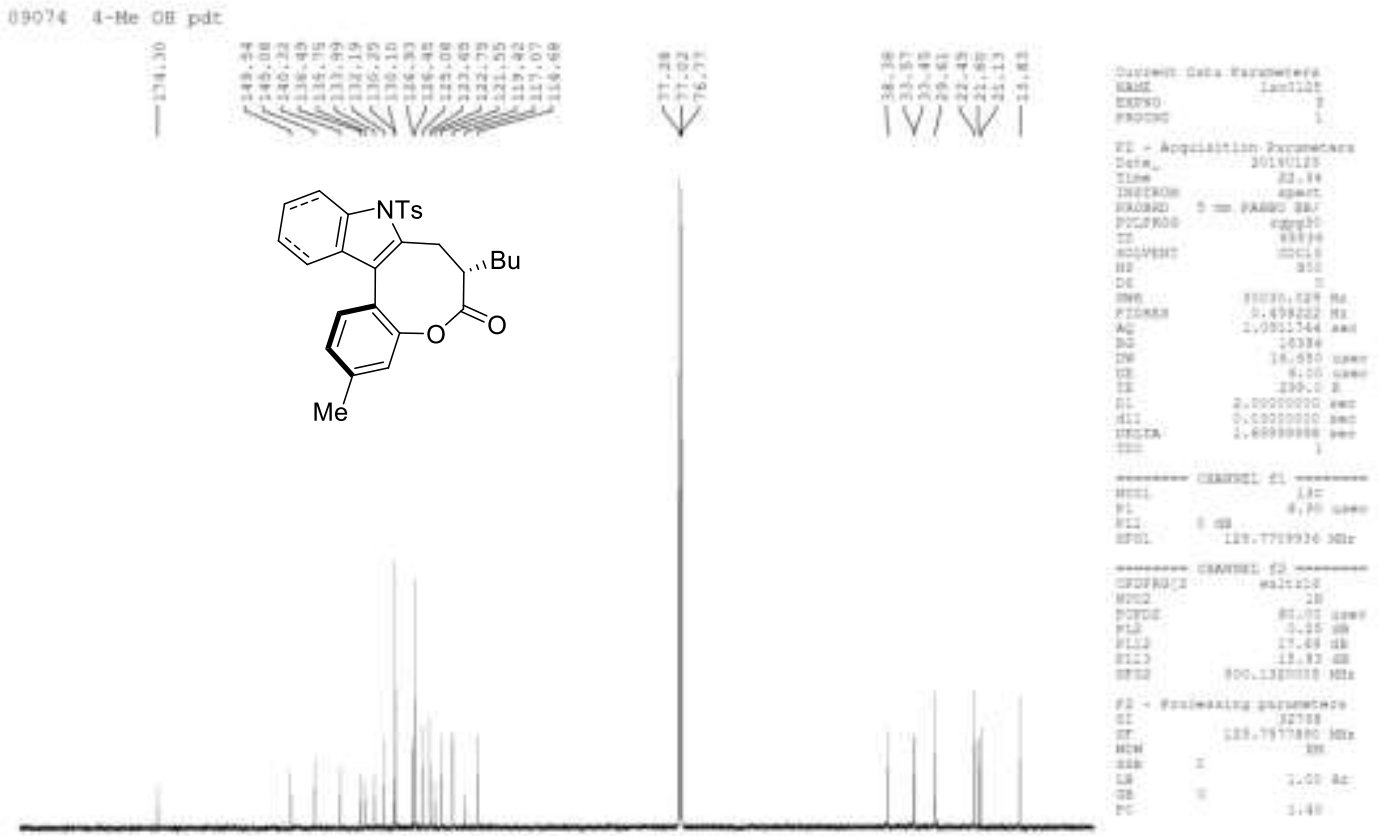

$\begin{array}{llllllllllllllllllll}190 & 180 & 170 & 160 & 150 & 140 & 130 & 120 & 110 & 100 & 00 & 80 & 70 & 60 & 50 & 40 & 30 & 20 & \text { ppm }\end{array}$ 
$(M, S)$-7-butyl-2,12-dimethyl-9-tosyl-7,8-dihydrobenzo[2,3] oxocino[5,4-b]indol-6(9H)-one. (Scheme 5, 6e)
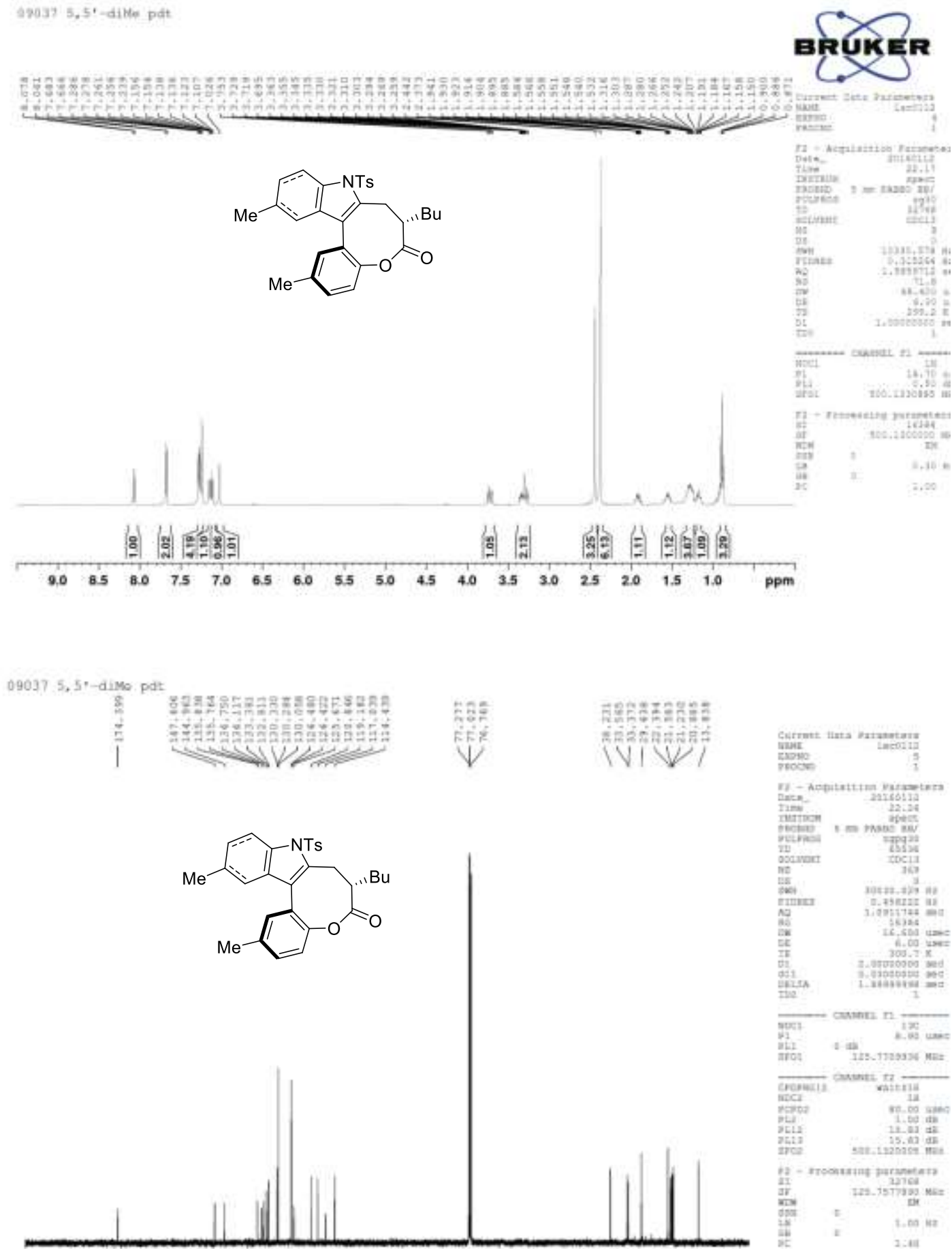

$\begin{array}{llllllllllllllllllllllllllll}190 & 180 & 170 & 160 & 150 & 140 & 130 & 120 & 110 & 100 & 90 & 80 & 70 & 60 & 50 & 40 & 30 & 20 & \text { ppm }\end{array}$ 
$(M, S)$-12-bromo-7-butyl-2-methyl-9-tosyl-7,8-dihydrobenzo[2,3] oxocino[5,4-b]indol-6(9H) -one. (Scheme 5, 6f)
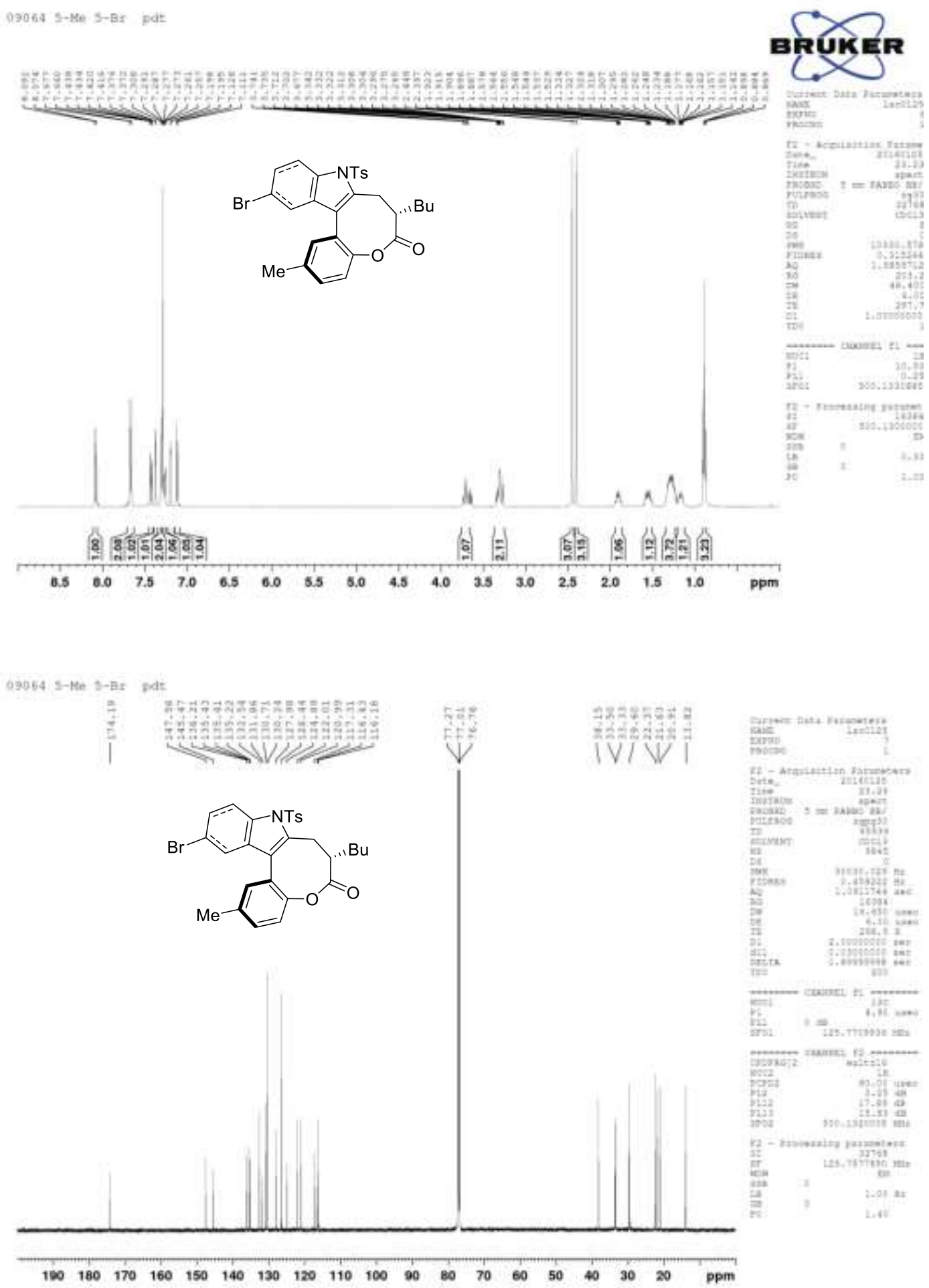
$(M, S)$-7-propyl-9-tosyl-7,8-dihydrobenzo[2,3] oxocino[5,4- $b]$ indol-6(9H)-one. (Scheme 5,

6g)

Irewat node in Et aldebivie for: $\mathrm{X}$-ray

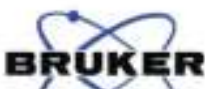

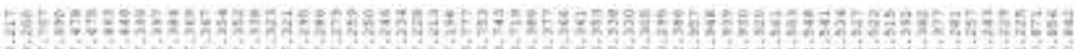
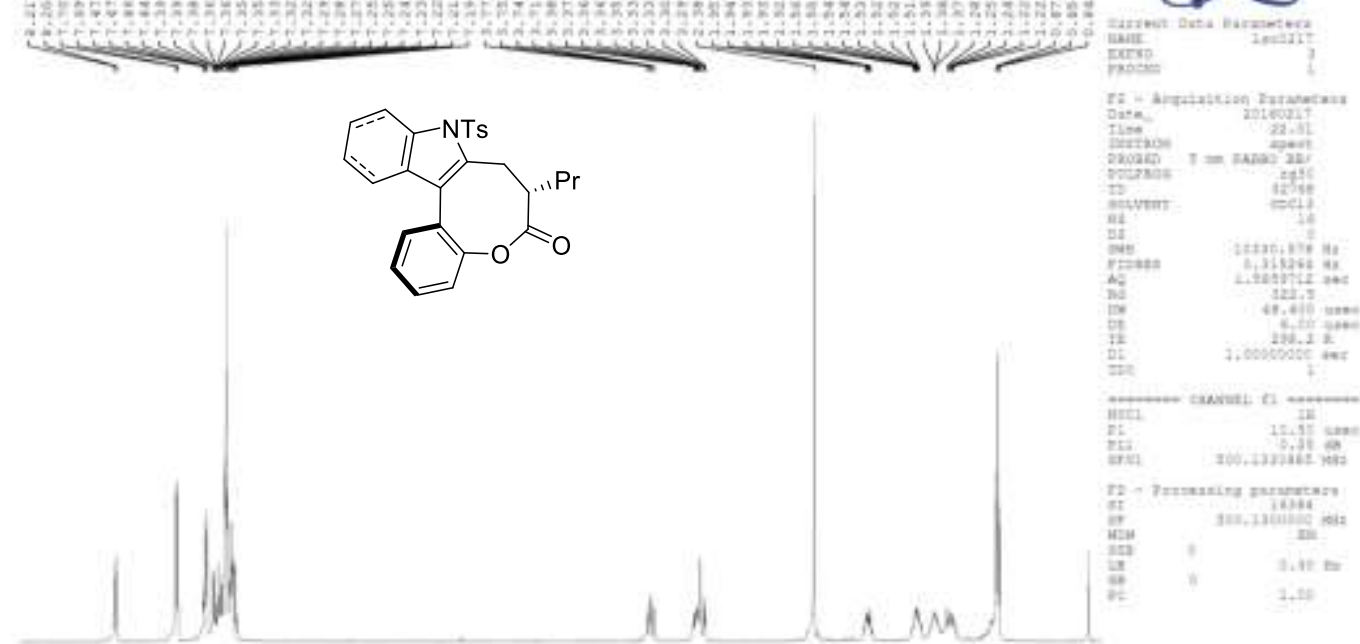

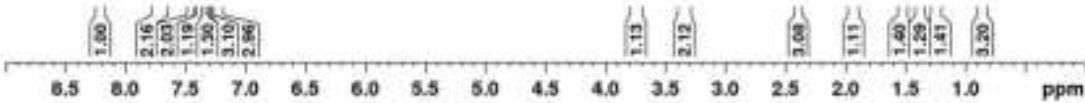

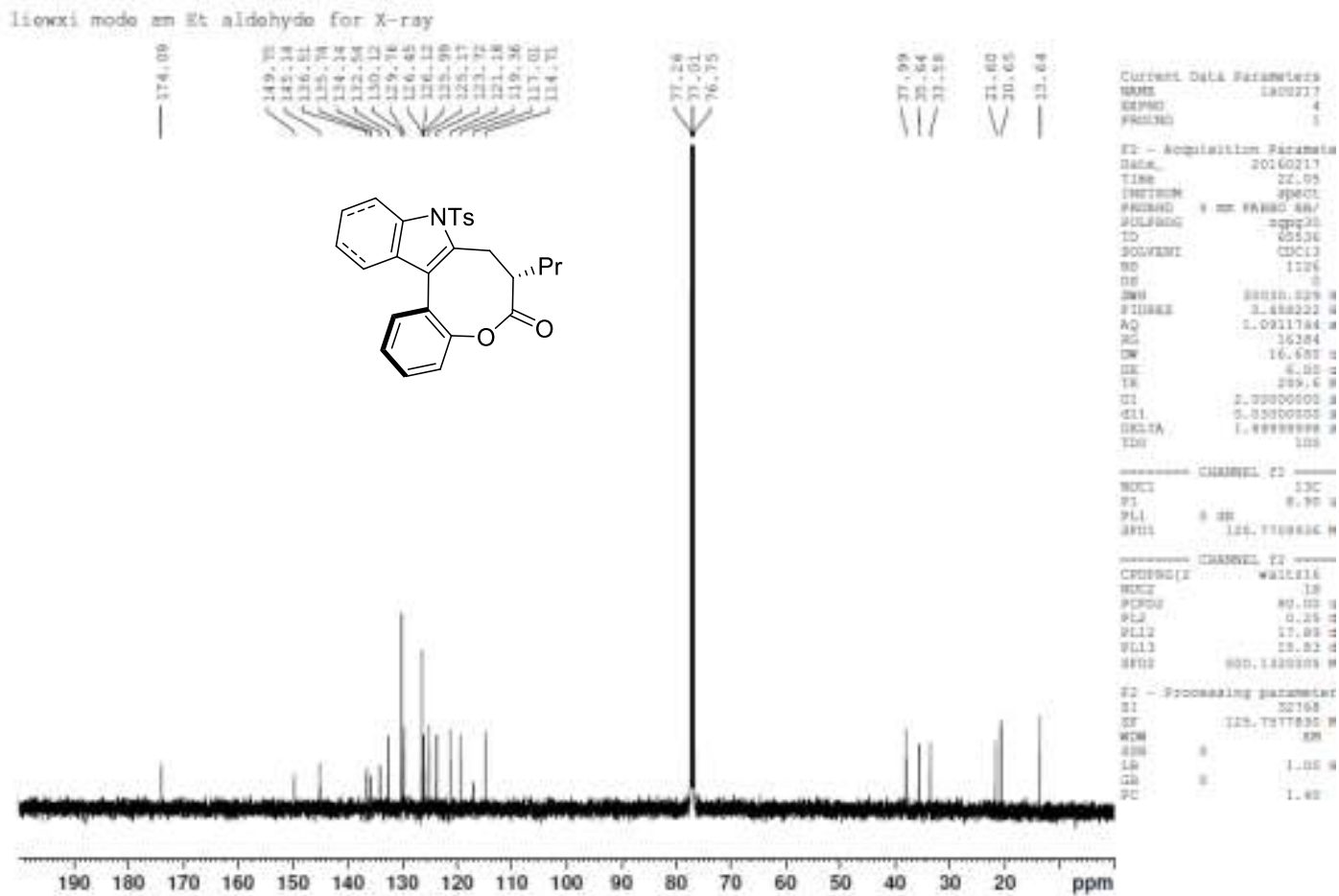


$(M, S)$-2,11-dimethyl-7-propyl-9-tosyl-7,8-dihydrobenzo[2,3] oxocino[5,4-b]indol-6(9H)-one. (Scheme 5, 6h)
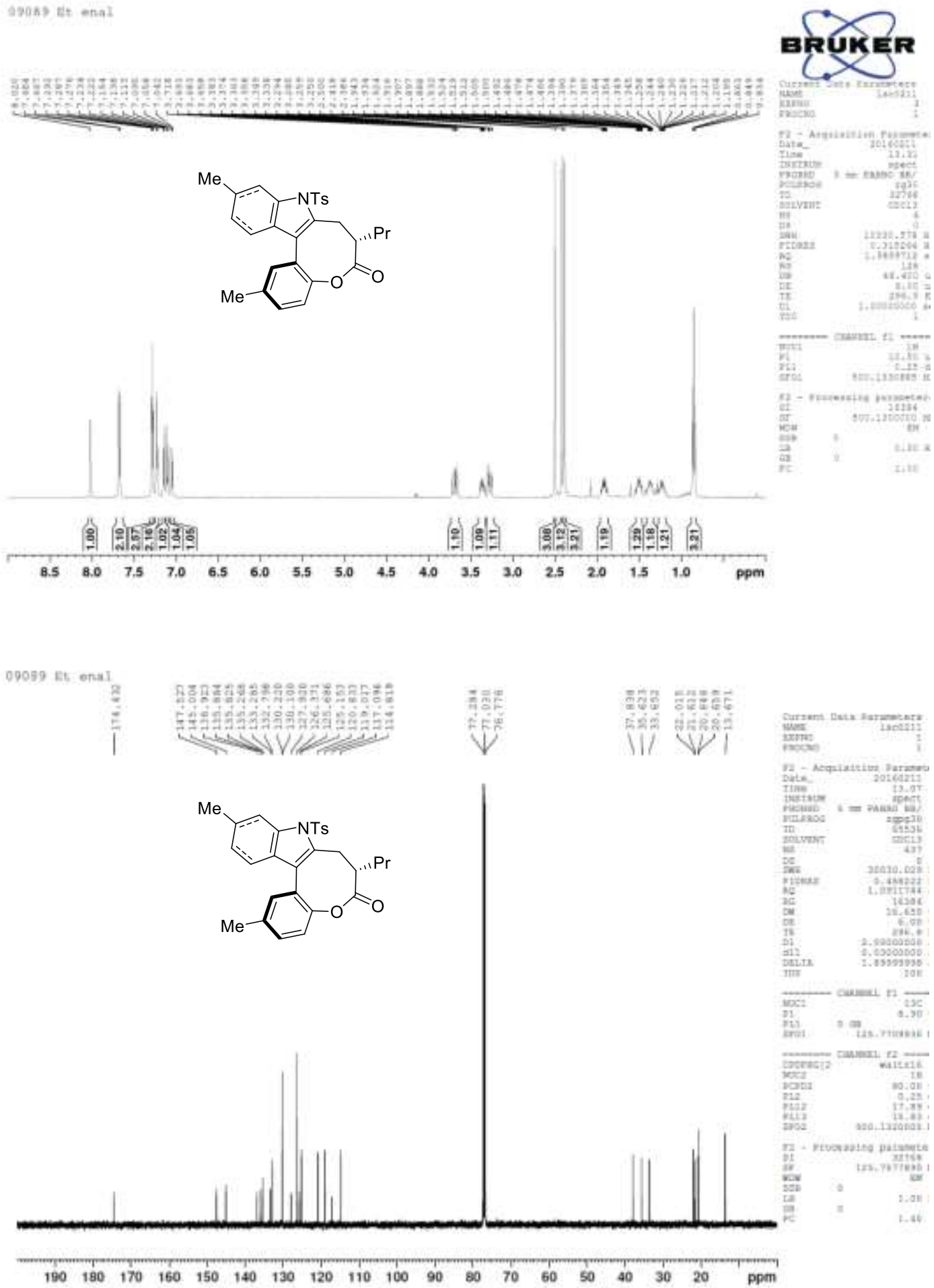
( $M, S$ )-7-butyl-2,11-dimethyl-9-tosyl-7,8-dihydrobenzo[2,3]oxocino[5,4- $b]$ indol-6(9H)-one. (Scheme 5, 6i)
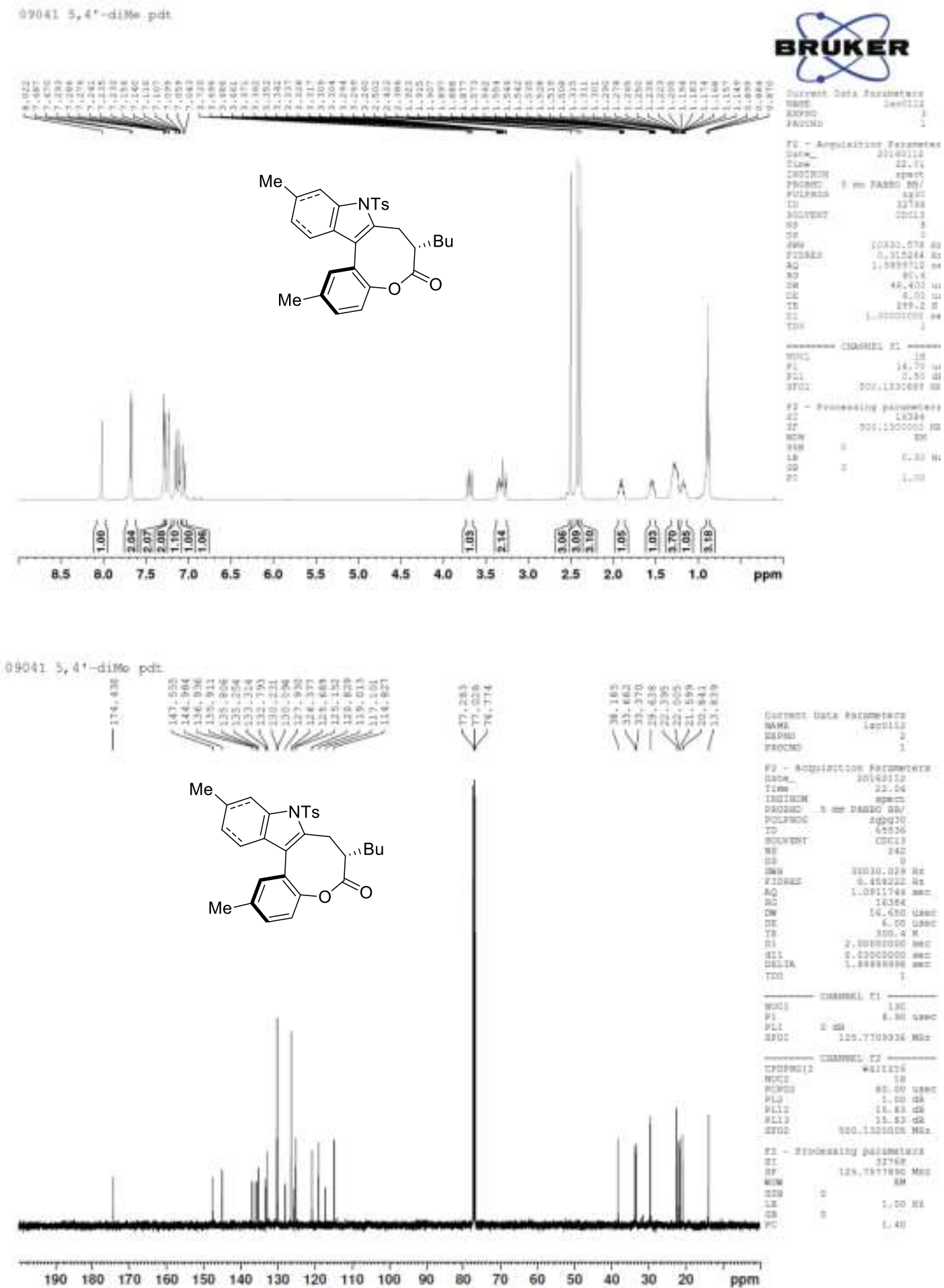
$(M, S)$-2,11-dimethyl-7-pentyl-9-tosyl-7,8-dihydrobenzo[2,3] oxocino[5,4- $b]$ indol-6(9H)-one. (Scheme 5, 6j)
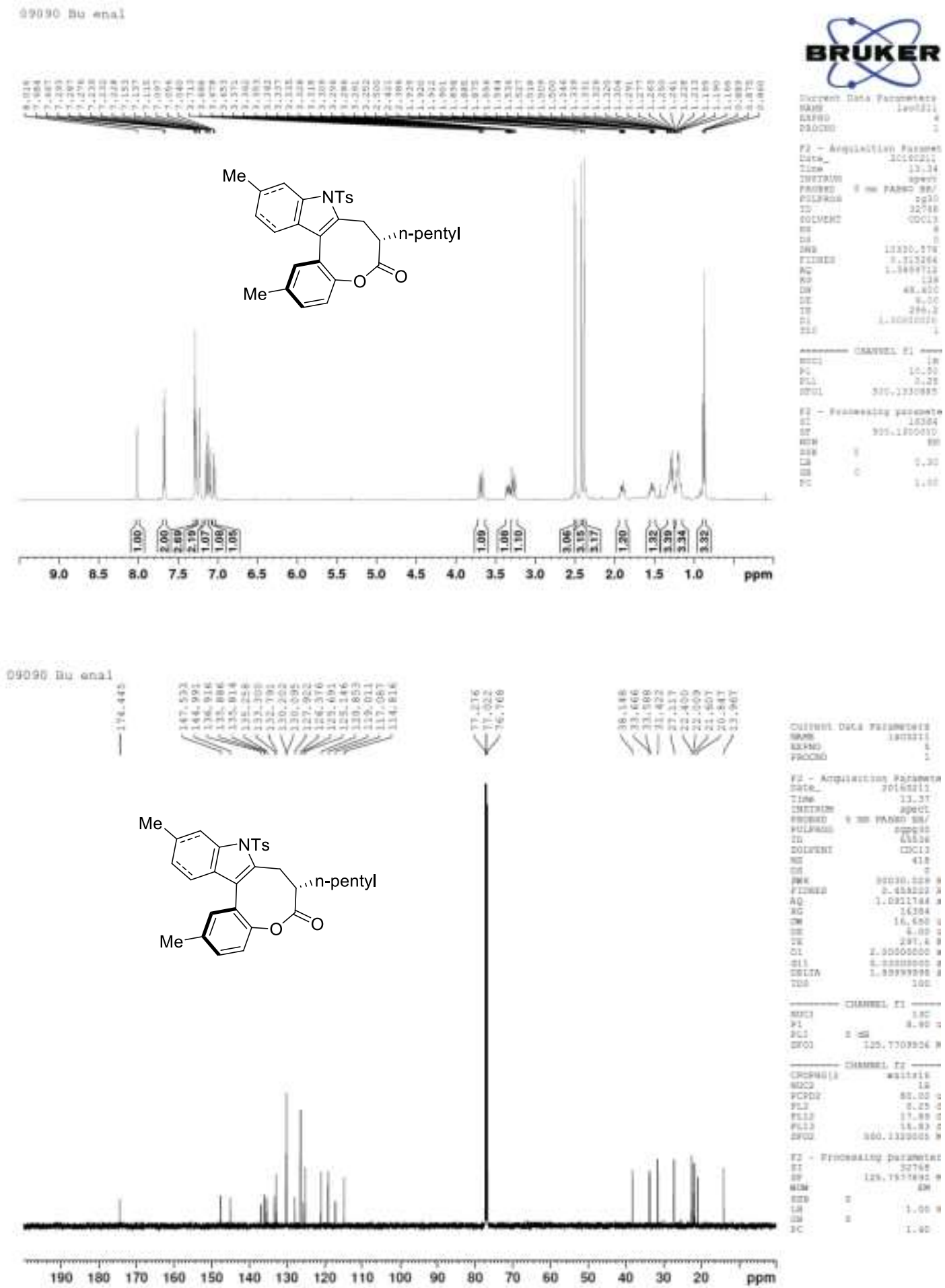
$(M, S)$-7-hexyl-2,11-dimethyl-9-tosyl-7,8-dihydrobenzo[2,3]oxocino[5,4-b]indol-6(9H)-one. (Scheme 5, 6k)
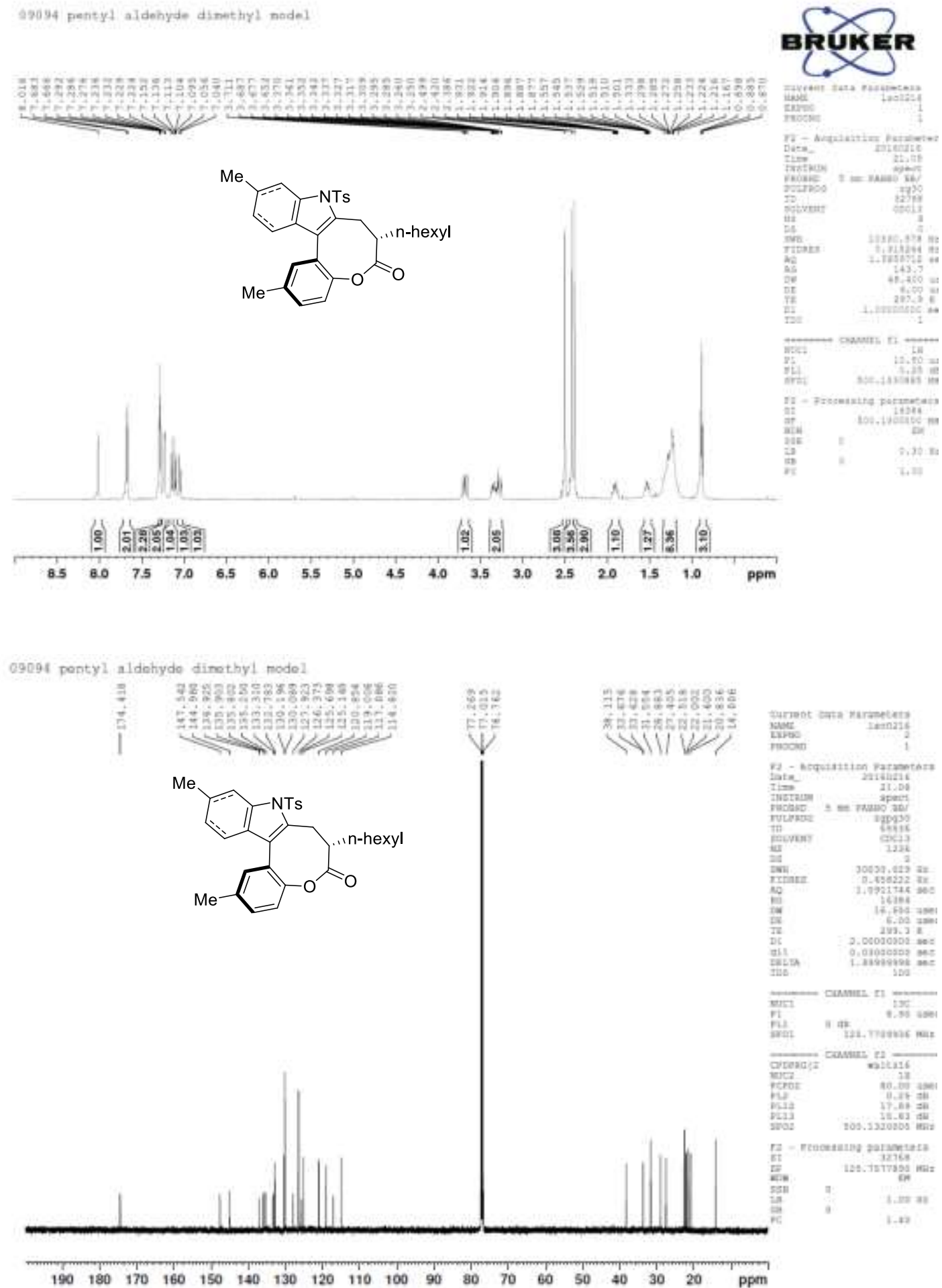
$(M, S)$-7-benzyl-2,11-dimethyl-9-tosyl-7,8-dihydrobenzo[2,3] oxocino[5,4-b]indol-6(9H)-one. (Scheme 5, 6l)
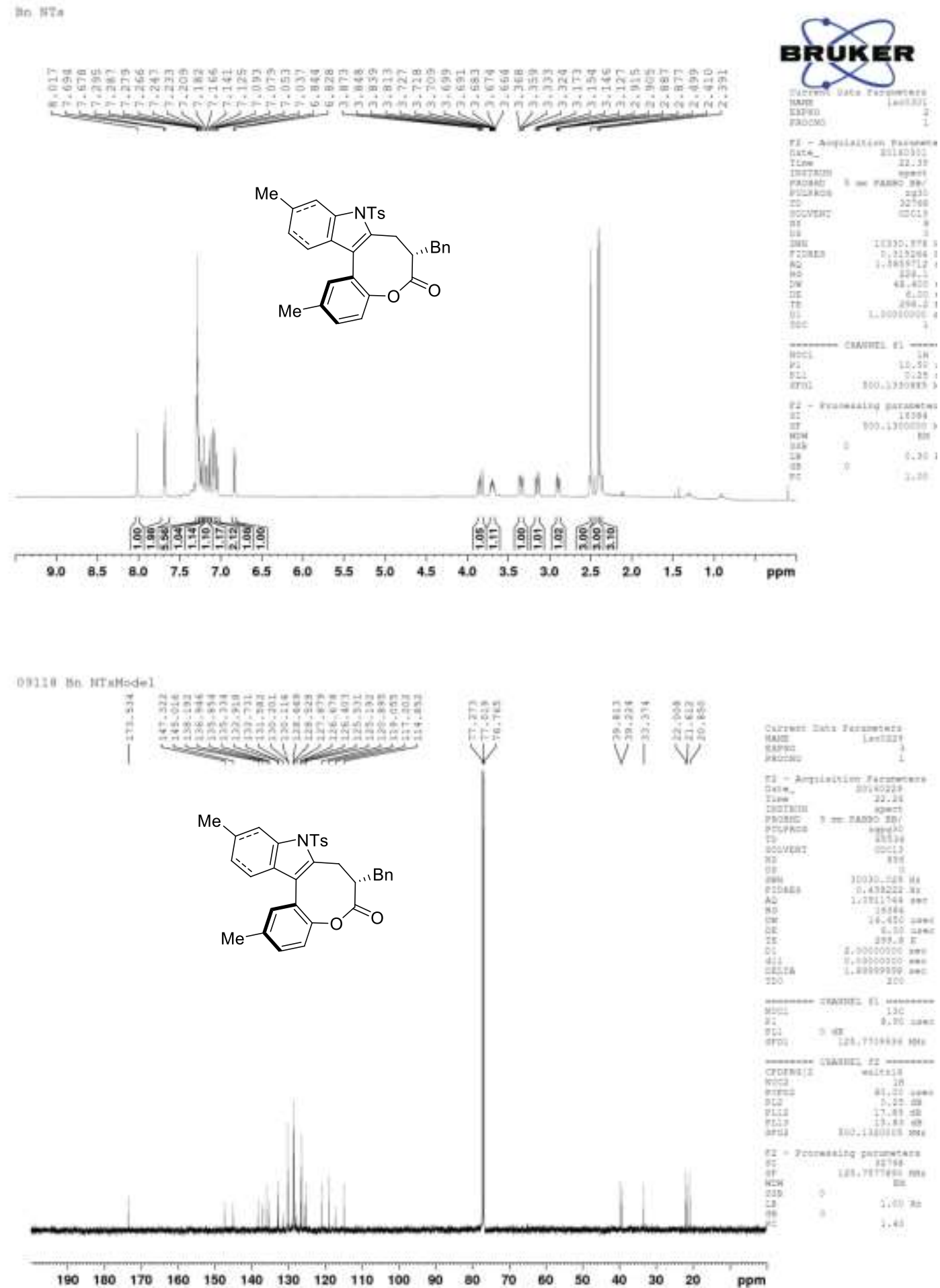
$(M, S)$-7-benzyl-2,11-dimethyl-9-tosyl-7,8-dihydrobenzo[2,3]oxocino[5,4-b]indol-6(9H)-one. (Scheme 5, 6m)
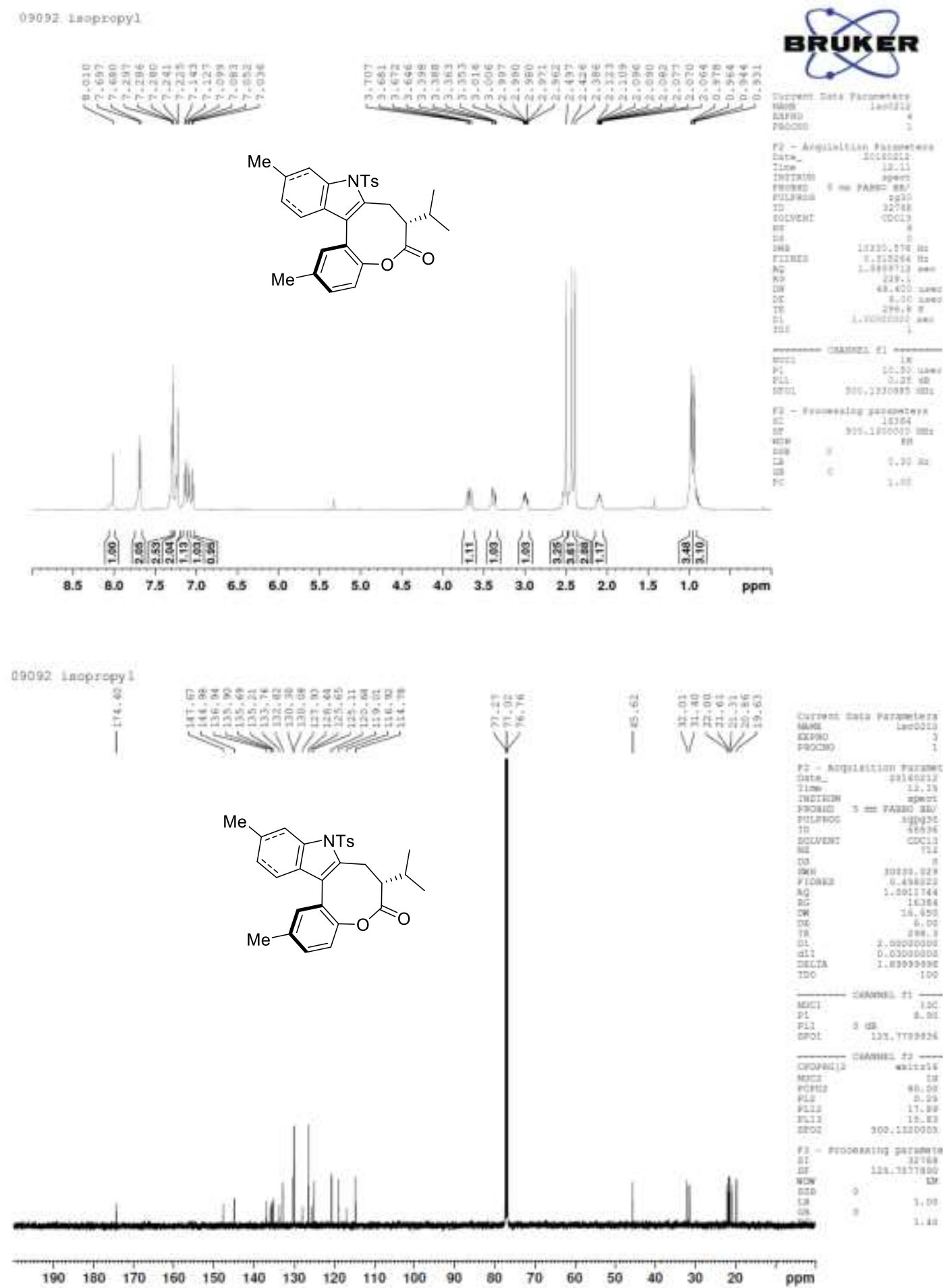
(M, S)-7-(3-((tert-butyldimethylsilyl)oxy)propyl)-2,11-dimethyl-9-tosyl-7,8-dihydrobenzo[2,3] oxocino[5,4-b]indol-6(9H)-one. (Scheme 5, 6n)
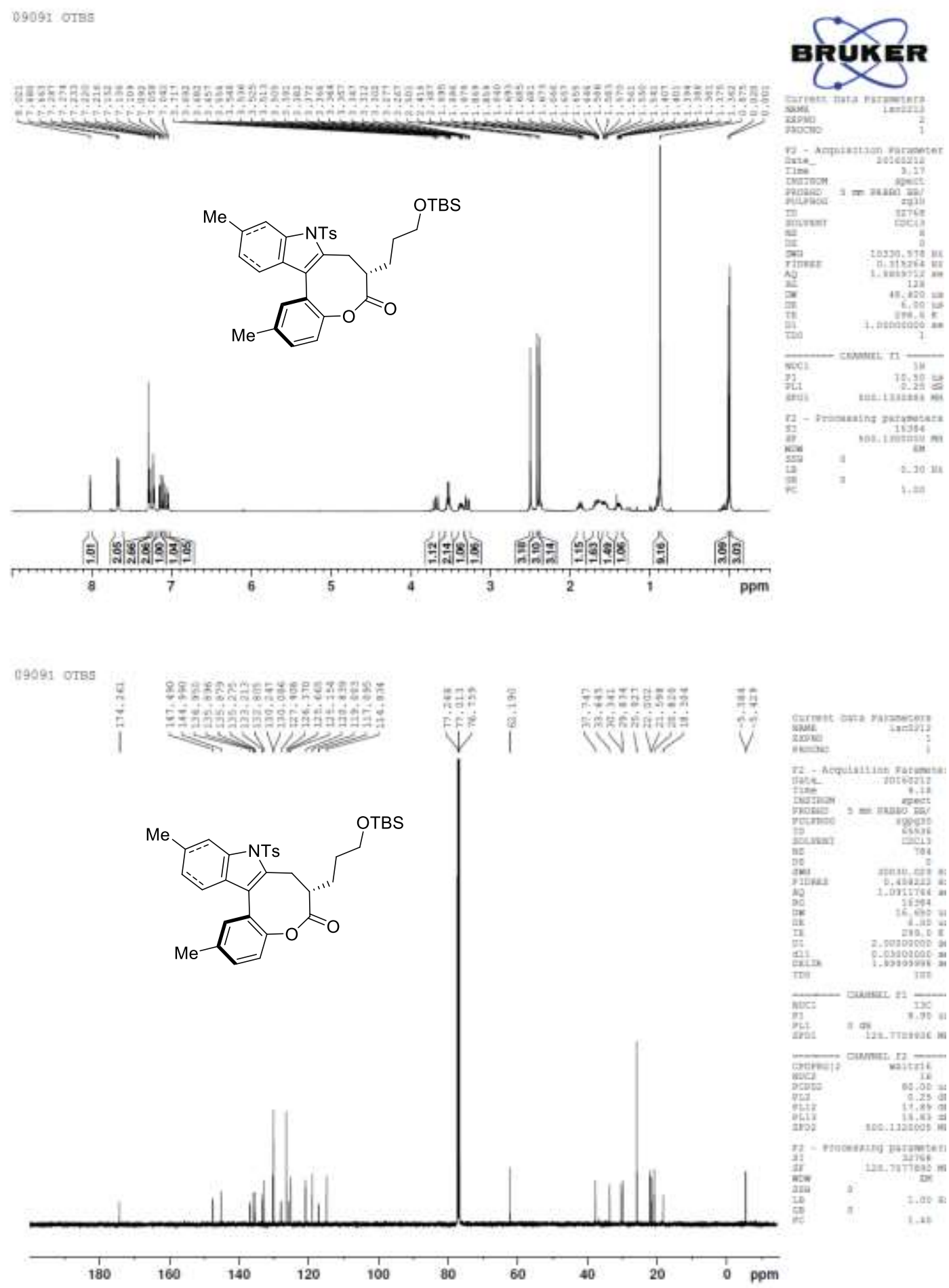


\section{D-NOESY Assignments}

(7R,8R)-7-isopropyl-8-methyl-7,8-dihydro-6H-benzo[2,3] oxocino[5,4-b]benzofuran-6-one . (Scheme 4a, 4r')
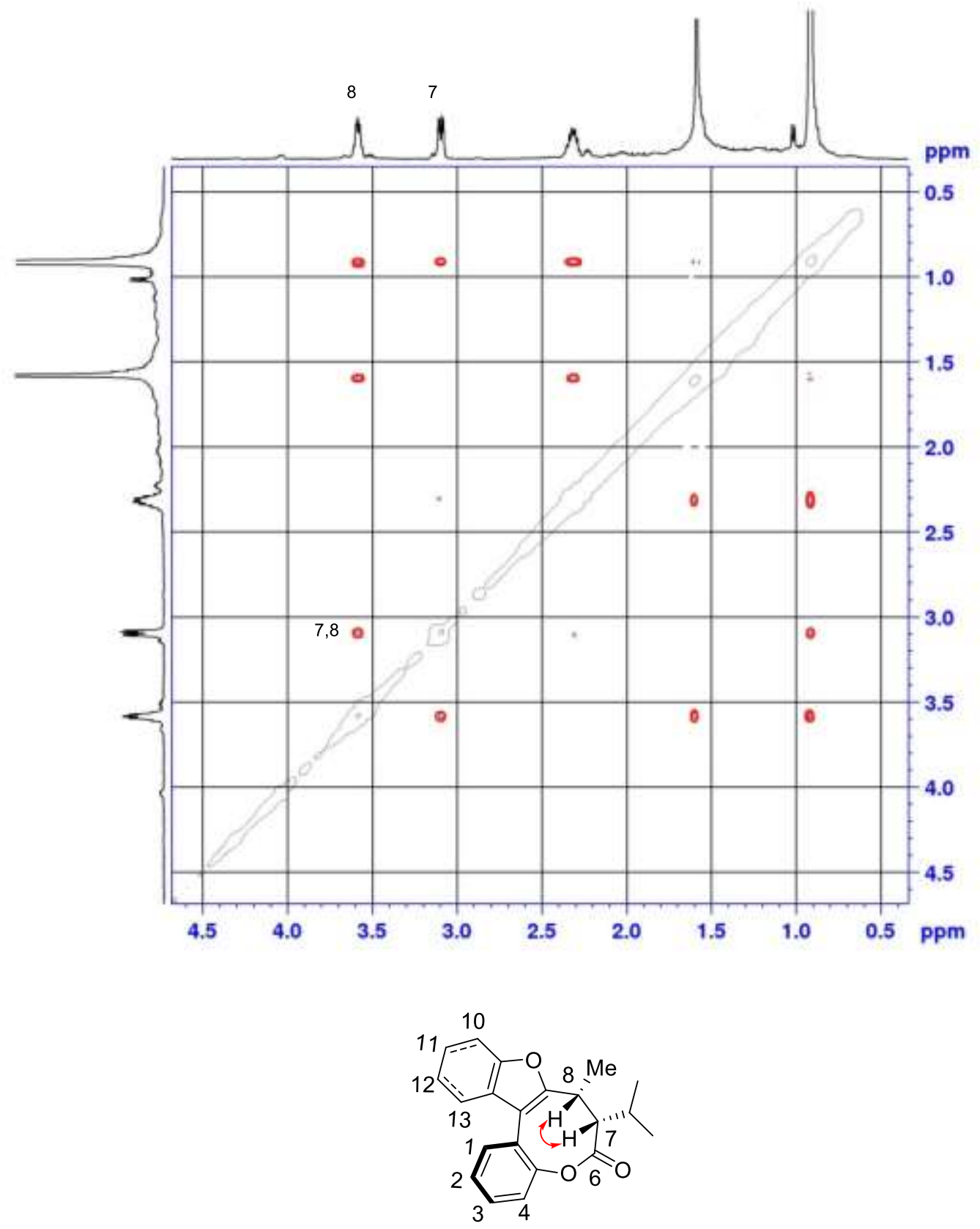
(S)-7-butyl-3-methoxy-7,8-dihydro-6H-benzo[2,3] oxocino[5,4-b]benzofuran-6-one. (Scheme $4 \mathrm{~b}, 4 \mathrm{~s})$

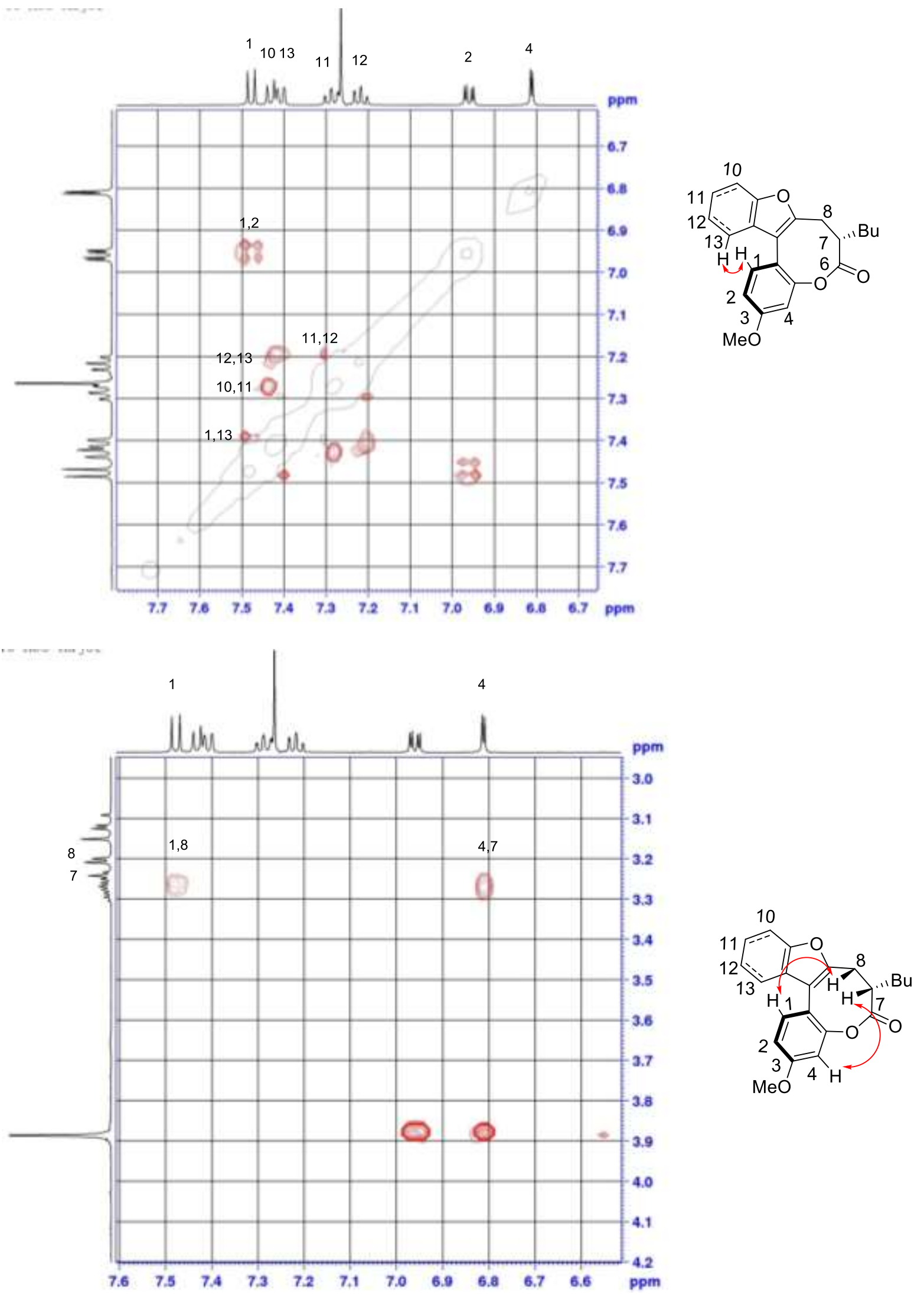


(S)-7-butyl-12-nitro-7,8-dihydro-6H-benzo[2,3] oxocino[5,4-b]benzofuran-6-one. (Scheme 4b, 4t)
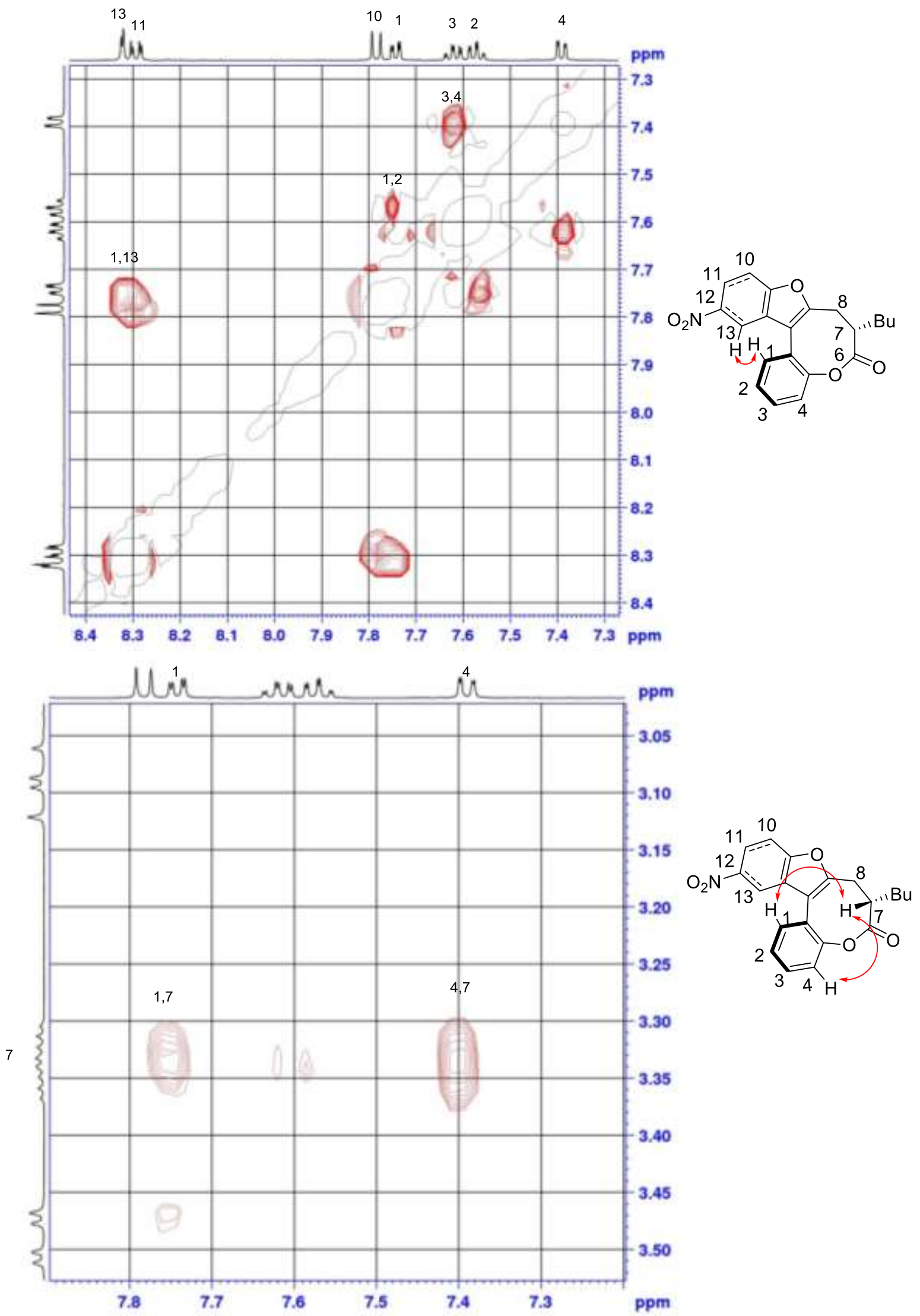
(M, S)-7-butyl-3-methyl-1-phenyl-7,8-dihydro-6H-benzo[b]benzofuro[3,2-d]oxocin-6-one

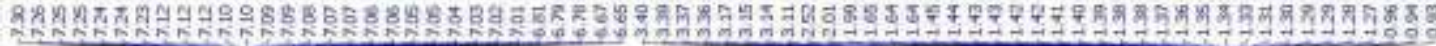

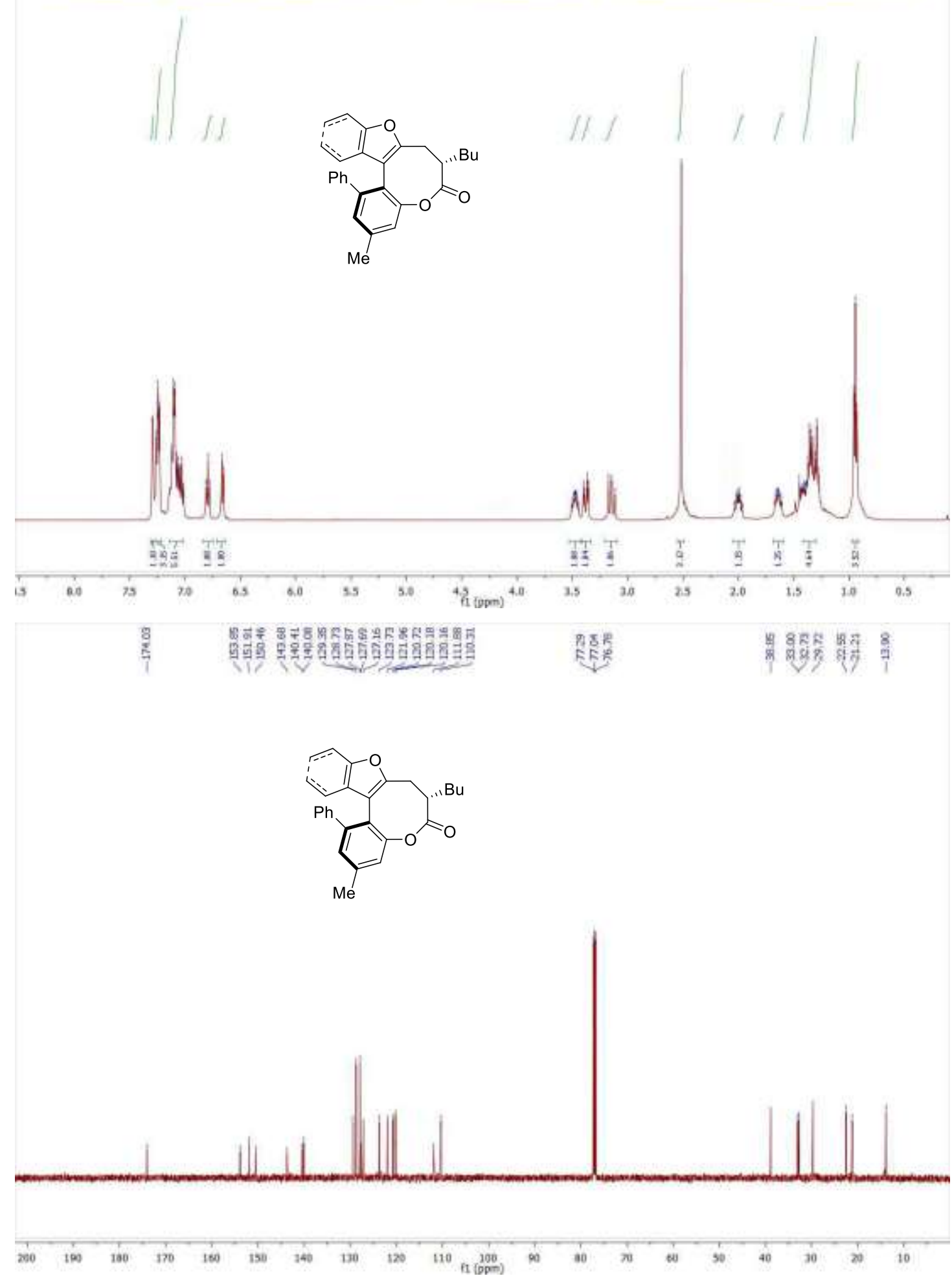


(M, S)-12-bromo-7-butyl-3-methyl-1-phenyl-7,8-dihydro-6H-benzo[b]benzofuro[3,2-d] oxocin-6 -one

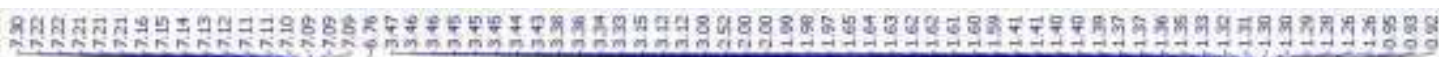<smiles>CC(C)Cc1cc([N+](=O)[O-])cc2c1-c1cc(Br)ccc1OC(=O)C2</smiles>
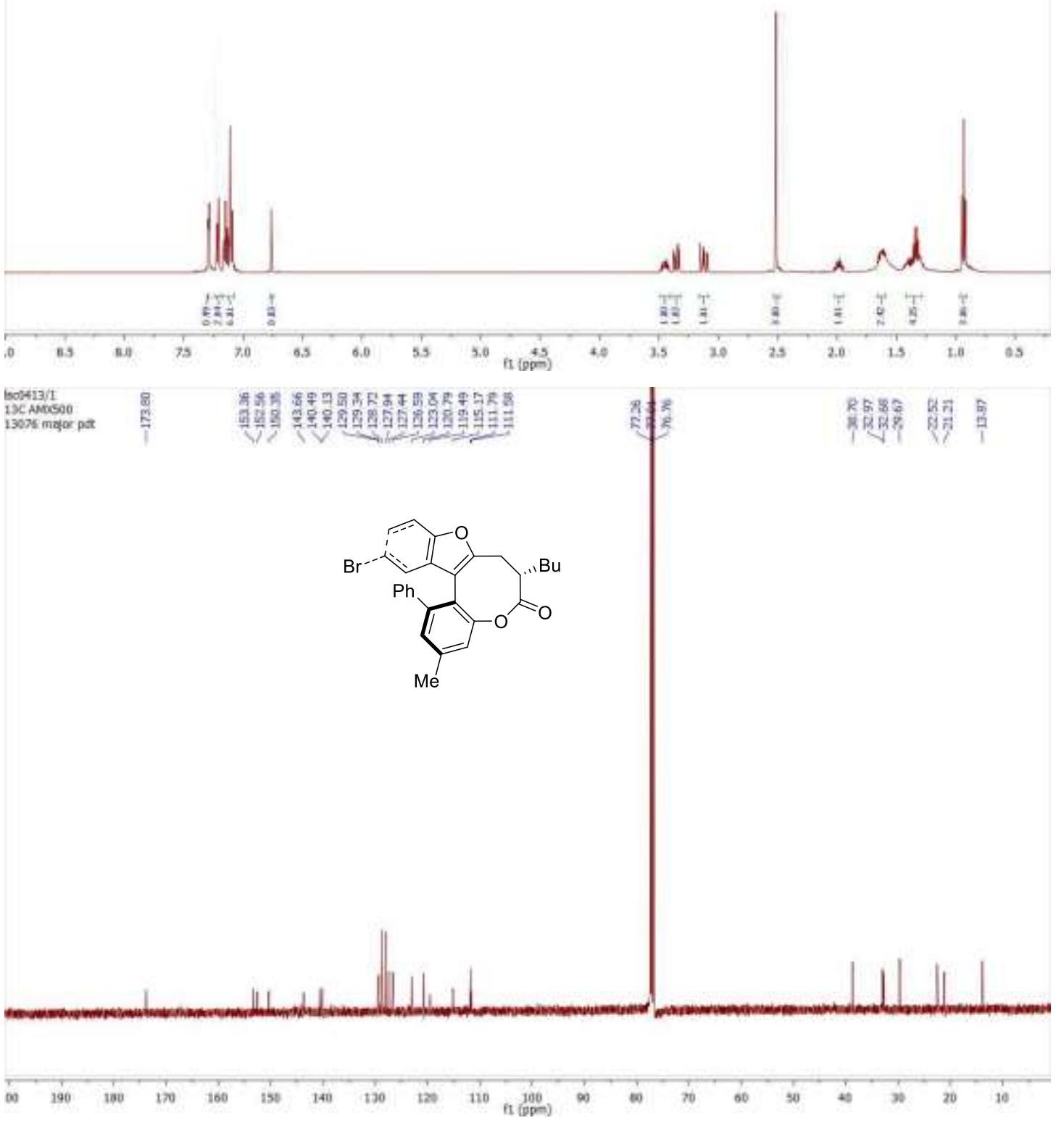
(M, S)-7-butyl-1,3-dimethyl-7,8-dihydro-6H-benzo[b]benzofuro[3,2-d]oxocin-6-one

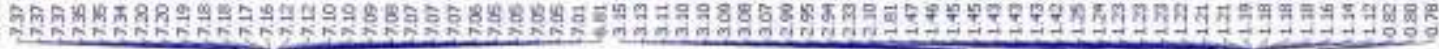<smiles>Cc1cc2c(c([N+](=O)[O-])c1)Cc1oc3ccccc3c1C(Br)CC2=O</smiles>

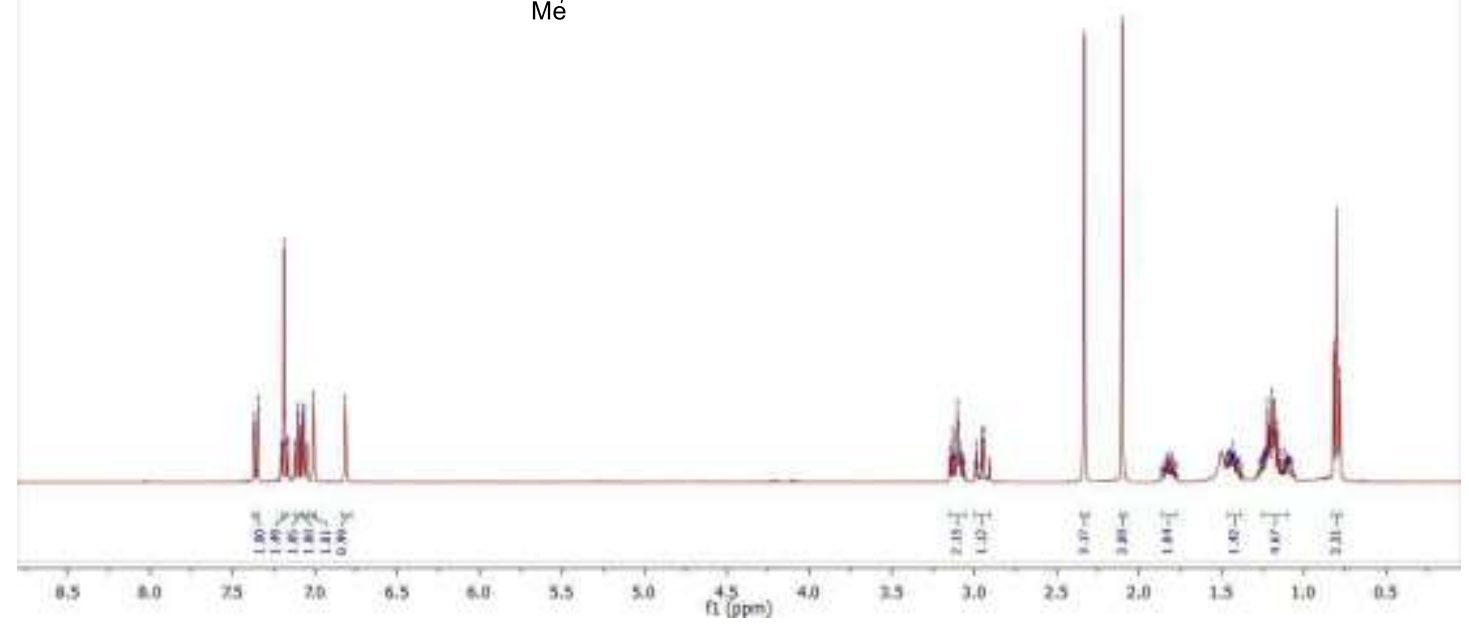

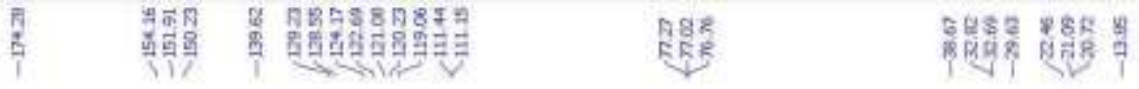

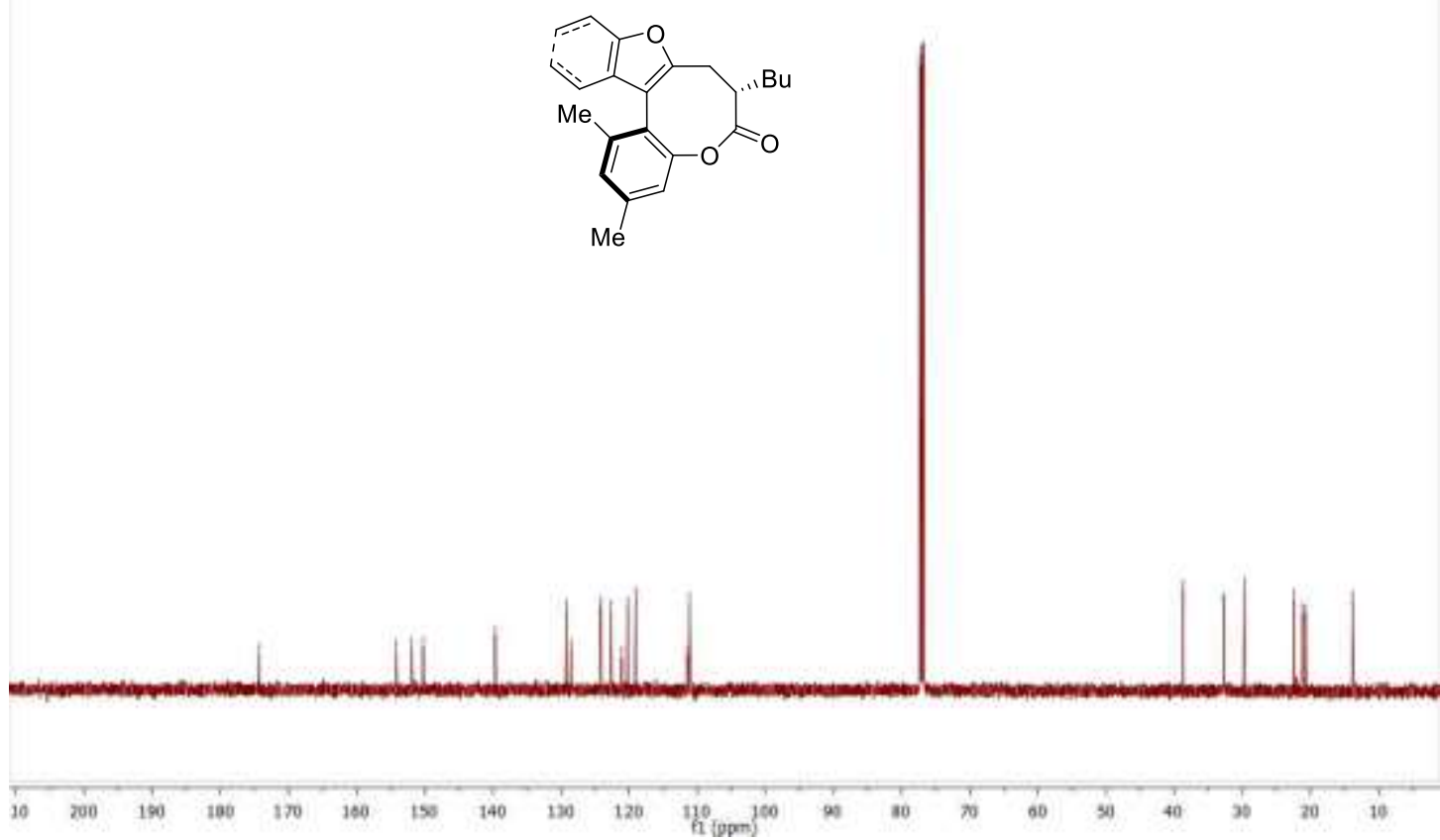


(S)-methyl 2-(((R)-3-(2-hydroxy-4,6-dimethylphenyl)benzofuran-2-yl)methyl)hexanoate

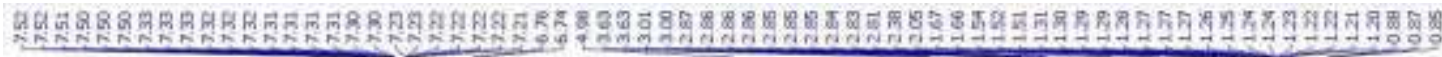<smiles>CC(=O)CC(Br)(Br)c1oc2ccccc2c1-c1c(O)cc([N+](=O)[O-])cc1[N+](=O)[O-]</smiles>

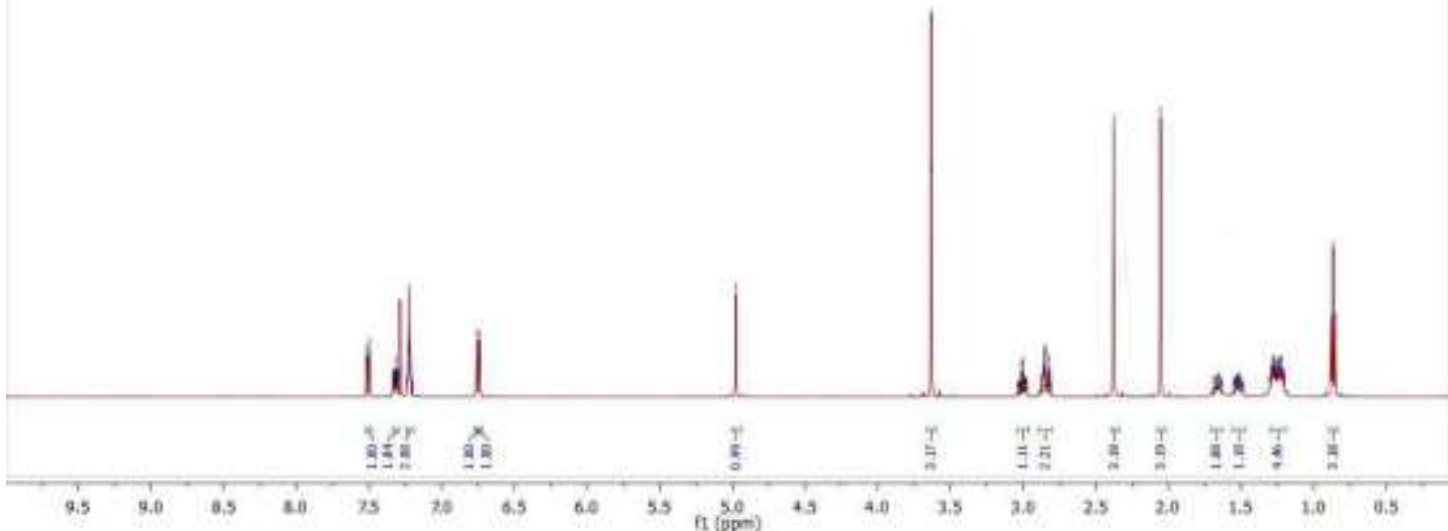

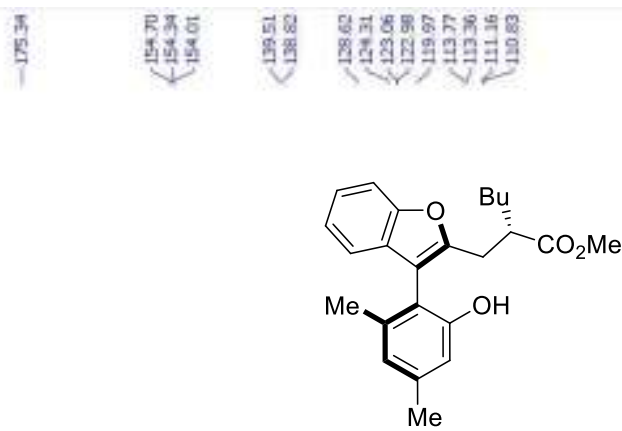
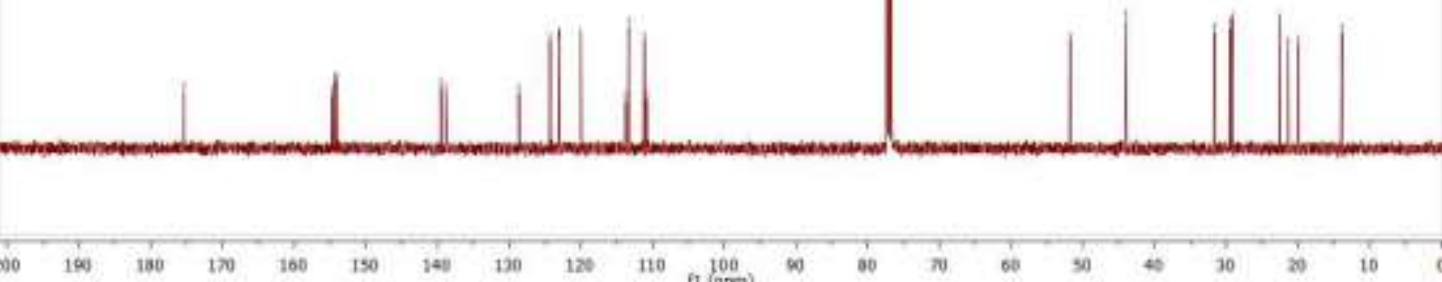


\section{Computational Data}

\subsection{Computational Details and References}

Full Reference of Gaussian09:

Gaussian 09, Revision B.01, M. J. Frisch, G. W. Trucks, H. B. Schlegel, G. E. Scuseria, M. A. Robb, J. R. Cheeseman, G. Scalmani, V. Barone, B. Mennucci, G. A. Petersson, H. Nakatsuji, M. Caricato, X. Li, H. P. Hratchian, A. F. Izmaylov, J. Bloino, G. Zheng, J. L. Sonnenberg, M. Hada, M. Ehara, K. Toyota, R. Fukuda, J. Hasegawa, M. Ishida, T. Nakajima, Y. Honda, O. Kitao, H. Nakai, T. Vreven, J. A. Montgomery, Jr., J. E. Peralta, F. Ogliaro, M. Bearpark, J. J. Heyd, E. Brothers, K. N. Kudin, V. N. Staroverov, T.Keith, R. Kobayashi, J. Normand, K. Raghavachari, A. Rendell, J. C. Burant, S. S. Iyengar, J. Tomasi, M. Cossi, N. Rega, J. M. Millam, M. Klene, J. E. Knox, J. B. Cross, V. Bakken, C. Adamo, J. Jaramillo, R. Gomperts, R. E. Stratmann, O. Yazyev, A. J. Austin, R. Cammi, C. Pomelli, J. W. Ochterski, R. L. Martin, K. Morokuma, V. G. Zakrzewski, G. A. Voth, P. Salvador, J. J. Dannenberg, S. Dapprich, A. D. Daniels, O. Farkas, J. B. Foresman, J. V. Ortiz, J. Cioslowski, and D. J. Fox, Gaussian, Inc., Wallingford CT, 2010.

All calculations were performed using the Gaussian 09 suite of programs. Equilibrium structures and transitions states were fully optimized using M06-2X density functional method with the 6-31G(d) basis sets, while single point energies were computed at M06-2X/6-311+G(2d, p) level. Frequency analyses at $298 \mathrm{~K}$ were performed on M06-2X/6-31G* optimized geometries to obtain thermodynamic corrections and to confirm the nature of the stationary points as equilibrium structures (with all real frequencies) or transition states (with only one imaginary frequency), and relative free energies, $\Delta \mathrm{G}_{298}$, were reported in all cases. For all calculations mentioned above, the solvent effect was modeled using the SMD model with tetrahydrofuran (THF) as the solvent.

\subsection{Alternative Mechanistic Pathway}

We proposed in Figure 4 of the main text that INT-2 first undergoes an eight-membered ring cyclization followed by a NHC-catalyzed nucleophilic addition to the internal allene moiety of INT-4 to afford product 4, shown in Figure 1S as pathway A. An alternative mechanistic pathway B, diverging from pathway A at INT-2, was also proposed and calculated. Starting from INT-2, the phenoxide moiety attacks the central carbon of the allene moiety to form a benzofuran intermedaite INT-3B through TS3B, with the help of the other phenol moiety to protonate the resulting carbanion. This step has a barrier of $14.7 \mathrm{kcal} / \mathrm{mol}$ and was calculated to be irreversible. Next, the phenoxide of INT-3B attacks the azolium moiety through TS4B to afford a tetrahedral intermediate INT-4B. Subsequent cleavage of C-C bond of INT-4B and leave of NHC catalyst through TS5B affords product 4. Compared to pathway A, which has three reversible steps before the irreversible formation of product $\mathbf{4}$, pathway B has an irreversible C-O bond formation step through TS3B. Giving the closeness of energies among the various relevant transition states of pathway $\mathrm{A}$ and $\mathrm{B}$, we were unable 
to rule out either pathway with confidence. Hence, our calculations suggested the reaction is likely to have two competitive pathways with a slight preference for the pathway A.
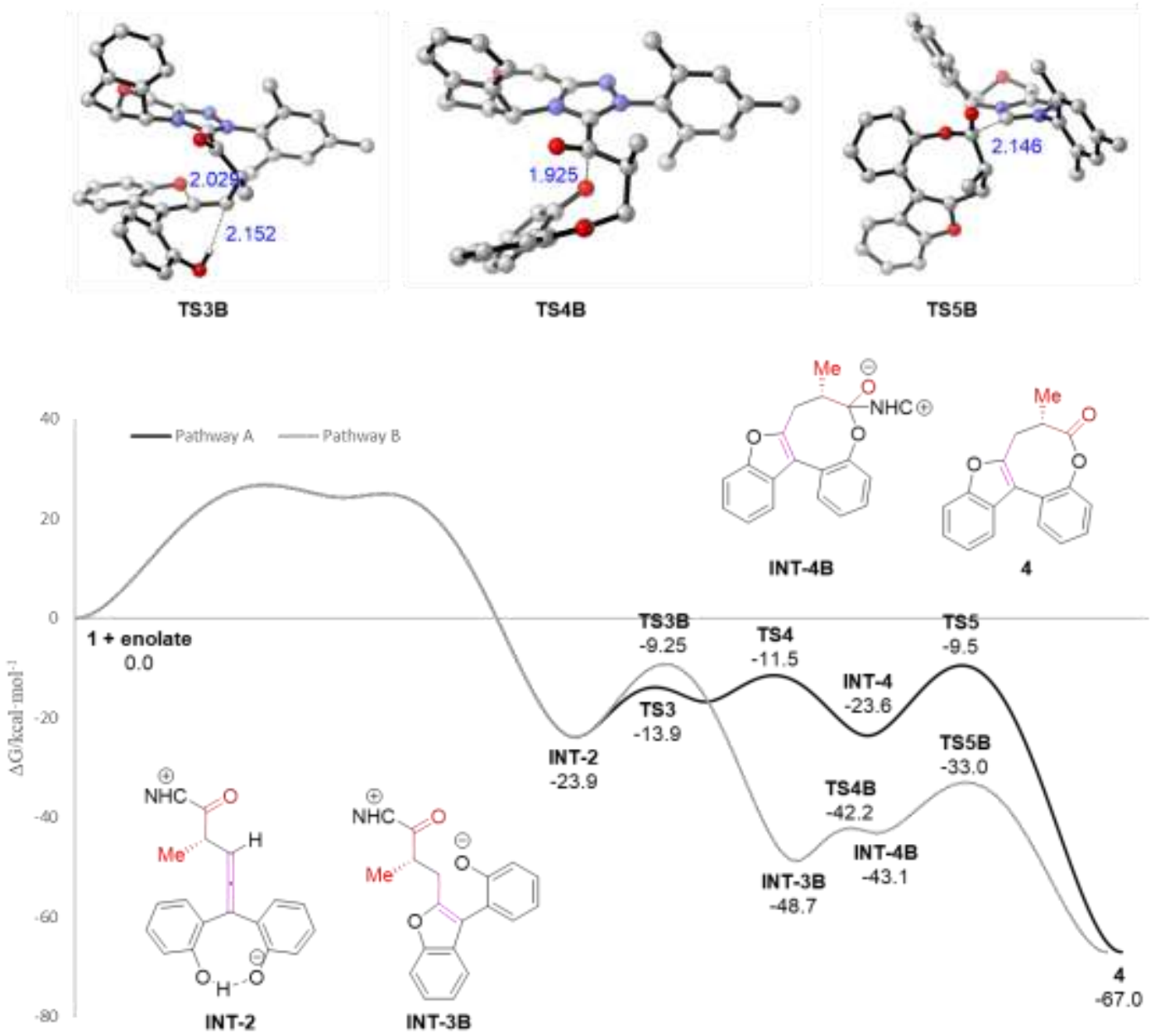

Figure 1S. | Two mechanistic pathways to form the cyclization product 4 from INT2. Relative free energies at $298 \mathrm{~K}, \Delta \mathrm{G}^{298}$, are reported in $\mathrm{kcal} / \mathrm{mol}$, and distances in $\AA$. For the shown TS structures, C-H hydrogen atoms are omitted for clarity.

\subsection{Origin of Enantioselectivity}

Our calculated reaction profiles, shown in Scheme 7 and Figure 1S, indicate that the enantioselectivity of the reaction is governed by the irreversible $\mathrm{C}-\mathrm{C}$ bond formation through TS1. To unravel the origin of enantioselectivity, we optimized different $S$-producing and $R$-producing TS1 structures. The lowest energy $S$-producing TS, namely TS1-S which is TS1 of Figure 4 renamed for the purpose of comparing for enantioselectivity, and the lowest energy $R$-producing TS1, namely TS1-R, are presented in Figure 2S. TS1-R was calculated to be $9.2 \mathrm{~kJ} / \mathrm{mol}$ higher in energy than TS1-S, in excellent agreement with experiments that the $S$ product is preferred. It is important to note the different conformations of the enolate moiety in the transition states. In the high energy TS1-R, the conformation of the enolate resulted in close proximity between the enolate and the catalyst moieties, as highlighted in Figure 2S. 


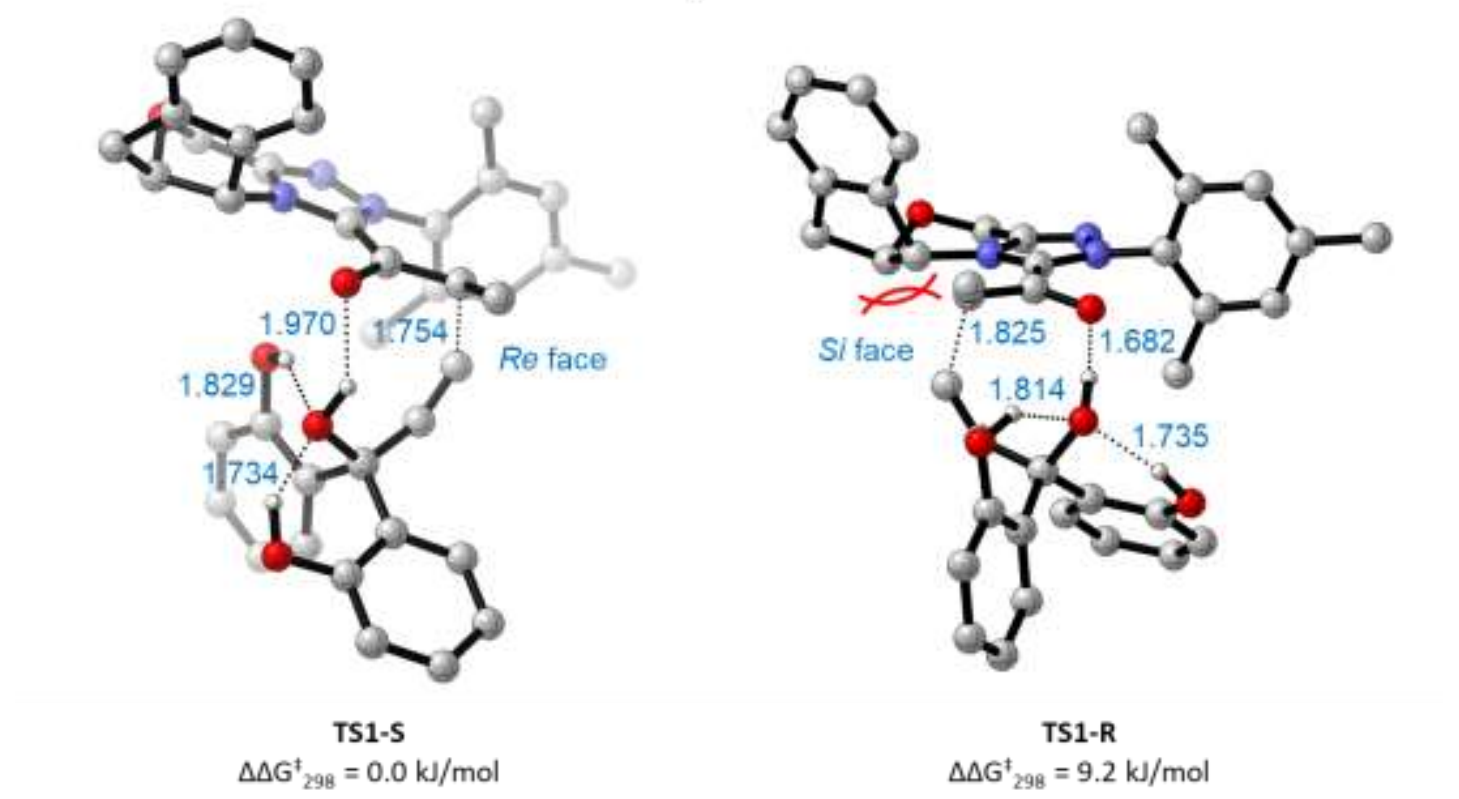

Figure 2S. | Optimized structures of the lowest-energy S-producing TS1-S and R-producing TS1-R, and their relative free energies calculated at M06-2X/6-311+G(2d,p)//M06-2X/6-31G* level. Distances are reported in $\AA$. C-H hydrogen atoms are omitted for clarity.

\subsection{Optimized Structures and Associated Thermodynamic Data}

\subsubsection{Pathway A and B}

$\begin{array}{crrr}\text { 1a } & & & \\ \mathrm{C} & -1.37844800 & 0.33548800 & 0.00670800 \\ \mathrm{C} & -3.90201000 & -0.81156500 & -0.37837200 \\ \mathrm{C} & -1.85293500 & -0.65205800 & 0.89140600 \\ \mathrm{C} & -2.18846600 & 0.73131900 & -1.05821600 \\ \mathrm{C} & -3.44445500 & 0.16760400 & -1.25810100 \\ \mathrm{C} & -3.11377500 & -1.21727500 & 0.69100200 \\ \mathrm{H} & -1.81969800 & 1.48800400 & -1.74378800 \\ \mathrm{H} & -4.05685400 & 0.48923300 & -2.09381800 \\ \mathrm{H} & -3.45165000 & -1.97573000 & 1.39008500 \\ \mathrm{H} & -4.87968800 & -1.26200400 & -0.52171000 \\ \mathrm{C} & 0.02871600 & 0.88710500 & 0.20674000 \\ \mathrm{C} & 1.12573400 & -0.13470000 & -0.11824200 \\ \mathrm{C} & 3.18120700 & -1.90522500 & -0.80819200 \\ \mathrm{C} & 0.85555600 & -1.29509600 & -0.84488700 \\ \mathrm{C} & 2.45380100 & 0.13217700 & 0.26694000 \\ \mathrm{C} & 3.47104700 & -0.75809600 & -0.08111700 \\ \mathrm{C} & 1.87034200 & -2.18101100 & -1.19153300\end{array}$




$\begin{array}{cccc}\mathrm{H} & -0.16539600 & -1.50176100 & -1.15043200 \\ \mathrm{H} & 4.48347100 & -0.52511100 & 0.23327700 \\ \mathrm{H} & 1.63657600 & -3.07721100 & -1.75634000 \\ \mathrm{H} & 3.98348400 & -2.58803700 & -1.07160100 \\ \mathrm{C} & 0.26458700 & 2.11156400 & -0.58596400 \\ \mathrm{H} & 0.68618500 & 4.01133400 & -1.76542000 \\ \mathrm{C} & 0.48379500 & 3.11933700 & -1.20884600 \\ 0 & 0.21455700 & 1.21659400 & 1.60814000 \\ \mathrm{H} & -0.42655100 & 1.91337300 & 1.84261000 \\ 0 & -1.12805700 & -1.11993600 & 1.93644400 \\ \mathrm{H} & -0.39225300 & -0.50592000 & 2.11691700 \\ 0 & 2.81917900 & 1.24428200 & 0.95172900 \\ \mathrm{H} & 2.02875700 & 1.62889900 & 1.37375200\end{array}$

Zero-point correction=

0.235282 (Hartree/Particle)

Thermal correction to Energy=

0.250218

Thermal correction to Enthalpy=

0.251162

Thermal correction to Gibbs Free Energy=

0.193619

Sum of electronic and zero-point Energies=

$-803.867229$

Sum of electronic and thermal Energies=

$-803.852294$

Sum of electronic and thermal Enthalpies=

$-803.851350$

Sum of electronic and thermal Free Energies=

$-803.908892$

$\mathrm{E}(\mathrm{RM} 062 \mathrm{X} / 6-311+\mathrm{G}(2 \mathrm{~d}, \mathrm{p}))=-804.367776314$

$\begin{array}{cccc}4 & & & \\ \mathrm{C} & 2.18923600 & -1.20789300 & 0.90285600 \\ 0 & 2.40324100 & -2.07371900 & 1.70908100 \\ \mathrm{C} & 2.16363000 & -1.45515100 & -0.59594900 \\ \mathrm{C} & 0.80263400 & -2.08596700 & -0.92921200 \\ \mathrm{C} & -0.36864600 & -1.25443500 & -0.52360800 \\ \mathrm{C} & -0.53692400 & 0.06288500 & -0.21620500 \\ \mathrm{C} & -1.96039900 & 0.22845300 & 0.02380300 \\ \mathrm{C} & -4.68387700 & -0.23641000 & 0.34436000 \\ \mathrm{C} & -2.78955300 & 1.29591600 & 0.38872200 \\ \mathrm{C} & -2.52345300 & -1.03755100 & -0.16135200 \\ \mathrm{C} & -3.87616600 & -1.31094100 & -0.01053000 \\ \mathrm{C} & -4.14740000 & 1.04788300 & 0.54233300 \\ \mathrm{H} & -2.38038000 & 2.28887600 & 0.55050100 \\ \mathrm{H} & -4.27218600 & -2.30950800 & -0.16046000 \\ \mathrm{H} & -4.80996900 & 1.86020200 & 0.82434700 \\ \mathrm{H} & -5.74985900 & -0.39433100 & 0.47432000 \\ \mathrm{C} & 0.44752100 & 1.16164700 & -0.19291900 \\ \mathrm{C} & 2.24135600 & 3.33427000 & -0.16106600 \\ \mathrm{C} & 1.63699700 & 1.11485700 & 0.54524900 \\ \mathrm{C} & 0.18172600 & 2.33545300 & -0.91227000\end{array}$




$\begin{array}{cccc}\mathrm{C} & 1.06288900 & 3.41099000 & -0.90038500 \\ \mathrm{C} & 2.52740100 & 2.18257800 & 0.56390100 \\ \mathrm{H} & -0.73377800 & 2.38955300 & -1.49473400 \\ \mathrm{H} & 0.83011200 & 4.30518100 & -1.46958900 \\ \mathrm{H} & 3.43144900 & 2.09707700 & 1.15811400 \\ \mathrm{H} & 2.93727000 & 4.16701300 & -0.14550200 \\ \mathrm{O} & -1.55481000 & -1.93416900 & -0.49330500 \\ \mathrm{O} & 1.93587200 & 0.04421300 & 1.37037800 \\ \mathrm{H} & 0.73022600 & -3.05470500 & -0.41988000 \\ \mathrm{H} & 0.75406400 & -2.28741300 & -2.00651500 \\ \mathrm{C} & 3.29622600 & -2.40486300 & -0.97876600 \\ \mathrm{H} & 4.27115800 & -1.96456600 & -0.75213200 \\ \mathrm{H} & 3.25538400 & -2.61727600 & -2.05089300 \\ \mathrm{H} & 3.20723900 & -3.34611800 & -0.43029000 \\ \mathrm{H} & 2.27567700 & -0.51491200 & -1.14027700\end{array}$

Zero-point correction=

0.280894 (Hartree/Particle)
0.296683
0.297627
0.237789
-919.406367
-919.390578
-919.389634
-919.449472

Thermal correction to Energy=

Thermal correction to Enthalpy=

Thermal correction to Gibbs Free Energy=

Sum of electronic and zero-point Energies=

Sum of electronic and thermal Energies=

$-919.449472$

Sum of electronic and thermal Free Energies=

$\mathrm{E}(\mathrm{RM} 062 \mathrm{X} / 6-311+\mathrm{G}(2 \mathrm{~d}, \mathrm{p}))=-919.960298170$

enolate

$\begin{array}{lrrc}\mathrm{C} & -0.19938600 & 0.01239000 & 0.55738200 \\ \mathrm{~N} & -1.13383500 & 0.70772900 & -0.10477300 \\ \mathrm{~N} & 0.94598400 & 0.71155500 & 0.36821800 \\ \mathrm{C} & 0.62970300 & 1.82698200 & -0.35910000 \\ \mathrm{~N} & -0.63046300 & 1.84628900 & -0.67057800 \\ \mathrm{C} & -2.51542300 & 0.39101900 & -0.34358300 \\ \mathrm{C} & -5.18206600 & -0.16047900 & -0.83871400 \\ \mathrm{C} & -2.82392500 & -0.61449100 & -1.26291800 \\ \mathrm{C} & -3.48422500 & 1.13841100 & 0.32993700 \\ \mathrm{C} & -4.82033300 & 0.84176100 & 0.06391600 \\ \mathrm{C} & -4.17432000 & -0.87297000 & -1.49278800 \\ \mathrm{H} & -5.59560900 & 1.40460000 & 0.57842900 \\ \mathrm{H} & -4.44435500 & -1.65150500 & -2.20264500 \\ \mathrm{C} & -3.09024000 & 2.20004100 & 1.32082400 \\ \mathrm{H} & -2.39480700 & 1.79792400 & 2.06671300 \\ \mathrm{H} & -3.97049100 & 2.58140700 & 1.84291100 \\ \mathrm{H} & -2.59167600 & 3.04056600 & 0.82658200 \\ \mathrm{C} & -1.74585800 & -1.40463000 & -1.95192900\end{array}$




\begin{tabular}{lrrr}
$\mathrm{H}$ & -1.29031200 & -2.11749500 & -1.25417300 \\
$\mathrm{H}$ & -0.95092800 & -0.75633400 & -2.33544600 \\
$\mathrm{H}$ & -2.16276900 & -1.97006600 & -2.78832000 \\
$\mathrm{C}$ & -6.63083500 & -0.49088600 & -1.08324400 \\
$\mathrm{H}$ & -6.94040800 & -1.34396600 & -0.46866500 \\
$\mathrm{H}$ & -6.80399800 & -0.76087800 & -2.12893000 \\
$\mathrm{H}$ & -7.27885400 & 0.35272400 & -0.83120600 \\
$\mathrm{C}$ & 2.31421000 & 0.43118200 & 0.83860000 \\
$\mathrm{H}$ & 2.23160000 & 0.05431900 & 1.85871900 \\
$\mathrm{C}$ & 3.15950800 & 1.72569300 & 0.77037900 \\
$\mathrm{H}$ & 2.94775500 & 2.41378800 & 1.59833000 \\
$\mathrm{C}$ & 1.64795600 & 2.89742900 & -0.61131900 \\
$\mathrm{H}$ & 1.47749600 & 3.72130200 & 0.09702800 \\
$\mathrm{H}$ & 1.54114700 & 3.28598300 & -1.62587600 \\
$\mathrm{O}$ & 2.94459900 & 2.37591900 & -0.47768200 \\
$\mathrm{C}$ & 4.59617900 & 1.20510700 & 0.72640700 \\
$\mathrm{H}$ & 4.95690400 & 1.00798000 & 1.74270600 \\
$\mathrm{H}$ & 5.26094700 & 1.93741400 & 0.26116100 \\
$\mathrm{C}$ & 4.44685100 & -0.07672100 & -0.06135100 \\
$\mathrm{C}$ & 3.69771300 & -2.38746100 & -1.40173000 \\
$\mathrm{C}$ & 5.40847000 & -0.79187000 & -0.76446800 \\
$\mathrm{C}$ & 3.12297300 & -0.52176700 & -0.02209200 \\
$\mathrm{C}$ & 2.73386200 & -1.67935600 & -0.68140900 \\
$\mathrm{C}$ & 5.02264200 & -1.95070800 & -1.44024400 \\
$\mathrm{H}$ & 6.43879200 & -0.44835000 & -0.79909300 \\
$\mathrm{H}$ & 1.71195900 & -2.03271000 & -0.60111600 \\
$\mathrm{H}$ & 5.75989200 & -2.51584900 & -2.00313900 \\
$\mathrm{H}$ & 3.41433400 & -3.29208200 & -1.93153200 \\
$\mathrm{C}$ & -0.28043900 & -1.23197800 & 1.40803500 \\
$\mathrm{C}$ & -1.51073100 & -1.50761200 & 1.94529600 \\
$\mathrm{H}$ & -2.36272900 & -0.87252500 & 1.73384700 \\
$\mathrm{C}$ & -1.69362300 & -2.66917300 & 2.87234300 \\
$\mathrm{H}$ & -2.44315900 & -3.38311300 & 2.50424400 \\
$\mathrm{H}$ & -2.02688700 & -2.35773300 & 3.87204900 \\
\hline & -0.74467200 & -3.20084300 & 2.98287100 \\
\hline & 0.83253100 & -1.82755000 & 1.57082000
\end{tabular}

Zero-point correction=

Thermal correction to Energy=

0.451098 (Hartree/Particle)
0.476803
0.477747
0.395070
-1243.333362
-1243.307657
-1243.306713
-1243.389390


$\mathrm{E}(\mathrm{RM} 062 \mathrm{X} / 6-311+\mathrm{G}(2 \mathrm{~d}, \mathrm{p}))=-1244.15139157$

INT-1

\begin{tabular}{|c|c|c|c|}
\hline $\mathrm{N}$ & 2.30412600 & 1.73958300 & 1.6010020 \\
\hline $\mathrm{C}$ & 2.76887900 & 0.52148700 & 1.6141060 \\
\hline $\mathrm{N}$ & 2.29052300 & -0.19756400 & 0.5614180 \\
\hline $\mathrm{C}$ & 1.45415900 & 0.61652400 & -0.1237640 \\
\hline $\mathrm{N}$ & 1.49423500 & 1.78563300 & 0.516515 \\
\hline $\mathrm{C}$ & 0.84562000 & 3.03981400 & 0.228529 \\
\hline $\mathrm{C}$ & -0.32206100 & 5.49229800 & -0.2422040 \\
\hline $\mathrm{C}$ & -0.24142400 & 3.40710900 & 1.0187350 \\
\hline $\mathrm{C}$ & 1.40263400 & 3.85284900 & -0.7651160 \\
\hline $\mathrm{C}$ & 0.78624300 & 5.07835400 & -0.9926910 \\
\hline $\mathrm{C}$ & -0.81428300 & 4.65419400 & 0.7567260 \\
\hline $\mathrm{H}$ & 1.18565000 & 5.73376700 & -1.7632910 \\
\hline $\mathrm{H}$ & -1.67434900 & 4.96635000 & 1.3439050 \\
\hline $\mathrm{C}$ & 2.61414600 & 3.41115300 & -1.54276100 \\
\hline $\mathrm{H}$ & 2.97620400 & 4.22355800 & -2.1762510 \\
\hline $\mathrm{H}$ & 2.38859100 & 2.55511400 & -2.1912230 \\
\hline $\mathrm{H}$ & 3.42609600 & 3.11051700 & -0.8711390 \\
\hline $\mathrm{C}$ & -0.77688200 & 2.50259700 & 2.0946190 \\
\hline $\mathrm{H}$ & -0.29430900 & 2.71739900 & 3.05538400 \\
\hline $\mathrm{H}$ & -0.60286300 & 1.45112800 & 9060 \\
\hline $\mathrm{H}$ & -1.85390400 & 2.64555600 & 2.2117130 \\
\hline $\mathrm{C}$ & -0.96142600 & 6.82695700 & -0.51922200 \\
\hline $\mathrm{H}$ & -0.23303300 & 7.63796000 & -0.4160060 \\
\hline $\mathrm{H}$ & -1.78939100 & 7.01963900 & 0.1671870 \\
\hline $\mathrm{H}$ & -1.34980800 & 6.86701700 & -1.54230500 \\
\hline $\mathrm{C}$ & 0.63519900 & 0.15458100 & -1.3136300 \\
\hline $\mathrm{C}$ & 2.65316300 & -1.61140500 & 0.3487520 \\
\hline $\mathrm{H}$ & 1.73373400 & -2.15787900 & 0.1367320 \\
\hline $\mathrm{C}$ & 3.32677500 & -2.18465000 & 1.6174350 \\
\hline $\mathrm{H}$ & 2.59337000 & -2.45036200 & 2.3886200 \\
\hline $\mathrm{C}$ & 3.64945400 & -0.10283100 & 2.6528420 \\
\hline $\mathrm{H}$ & 3.03580300 & -0.34184700 & 3.5335420 \\
\hline $\mathrm{H}$ & 4.42830200 & 0.60231600 & 2.948463 \\
\hline 0 & 4.26688600 & -1.25090400 & 2.13106800 \\
\hline $\mathrm{C}$ & 4.13147800 & -3.36688000 & 1.0738940 \\
\hline $\mathrm{H}$ & 4.97713200 & -3.59387200 & $1.727850 c$ \\
\hline $\mathrm{H}$ & 3.49395000 & -4.25646200 & 1.0147910 \\
\hline $\mathrm{C}$ & 3.70045100 & -1.84701500 & -0.7204620 \\
\hline $\mathrm{C}$ & 5.76404300 & -2.70373100 & -2.35186100 \\
\hline $\mathrm{C}$ & 3.89348400 & -1.21754300 & -1.9421780 \\
\hline $\mathrm{C}$ & 4.53371800 & -2.88804600 & -0.3025210 \\
\hline
\end{tabular}




\begin{tabular}{|c|c|c|c|}
\hline $\mathrm{C}$ & 5.56691900 & -3.32879100 & -1.11927100 \\
\hline $\mathrm{C}$ & 4.94025000 & -1.65346300 & -2.75665600 \\
\hline $\mathrm{H}$ & 3.25640500 & -0.39950600 & -2.26428800 \\
\hline $\mathrm{H}$ & 6.22011900 & -4.13533500 & -0.79805200 \\
\hline $\mathrm{H}$ & 5.11310800 & -1.16903100 & -3.71269900 \\
\hline $\mathrm{H}$ & 6.57357500 & -3.03106600 & -2.99734100 \\
\hline 0 & 0.81231200 & -1.01092600 & -1.63690400 \\
\hline $\mathrm{C}$ & -0.34371900 & 1.05683700 & -1.96457700 \\
\hline $\mathrm{C}$ & -0.77199300 & 0.51152100 & -3.32589600 \\
\hline $\mathrm{H}$ & -1.38235100 & -0.39224600 & -3.22769100 \\
\hline $\mathrm{H}$ & 0.08565400 & 0.27418600 & -3.96374700 \\
\hline $\mathrm{H}$ & -1.38942000 & 1.25990400 & -3.82931800 \\
\hline $\mathrm{H}$ & 0.15327000 & 2.02109500 & -2.10558000 \\
\hline $\mathrm{H}$ & -1.70096600 & 2.48926000 & -1.04726200 \\
\hline $\mathrm{C}$ & -1.60625300 & 1.40040200 & -1.06195700 \\
\hline $\mathrm{C}$ & -2.54786400 & 0.66354800 & -0.48369500 \\
\hline $\mathrm{C}$ & -2.74019600 & -0.76286100 & -0.25006100 \\
\hline $\mathrm{C}$ & -4.01658000 & -1.29946300 & -0.90873100 \\
\hline $\mathrm{C}$ & -6.40959000 & -2.29050500 & -2.01578400 \\
\hline $\mathrm{C}$ & -4.94489200 & -0.44924600 & -1.51378200 \\
\hline $\mathrm{C}$ & -4.30740800 & -2.68069000 & -0.88264100 \\
\hline $\mathrm{C}$ & -5.50105400 & -3.16168300 & -1.42682100 \\
\hline $\mathrm{C}$ & -6.12779300 & -0.92745100 & -2.07180600 \\
\hline $\mathrm{H}$ & -4.72577900 & 0.61330300 & -1.51658100 \\
\hline $\mathrm{H}$ & -5.68926800 & -4.23003000 & -1.38064700 \\
\hline $\mathrm{H}$ & -6.82724700 & -0.23727700 & -2.53313900 \\
\hline $\mathrm{H}$ & -7.33374700 & -2.67964600 & -2.43377700 \\
\hline $\mathrm{C}$ & -2.74031100 & -0.95580700 & 1.27920000 \\
\hline $\mathrm{C}$ & -2.73290700 & -1.02608200 & 4.09256400 \\
\hline $\mathrm{C}$ & -3.93189900 & -0.95123900 & 2.00606400 \\
\hline $\mathrm{C}$ & -1.53153100 & -1.00678600 & 1.99734700 \\
\hline $\mathrm{C}$ & -1.53223500 & -1.03773500 & 3.39272600 \\
\hline $\mathrm{C}$ & -3.94043400 & -0.99059800 & 3.39735300 \\
\hline $\mathrm{H}$ & -4.87164600 & -0.90072600 & 1.46297900 \\
\hline $\mathrm{H}$ & -0.57592000 & -1.07403500 & 3.90677300 \\
\hline $\mathrm{H}$ & -4.88434000 & -0.98518000 & 3.93324300 \\
\hline $\mathrm{H}$ & -2.72396300 & -1.05079300 & 5.17838200 \\
\hline 0 & -1.63814900 & -1.74962100 & -0.68689900 \\
\hline $\mathrm{H}$ & -1.39243800 & -1.54892400 & -1.60480800 \\
\hline 0 & -3.47219100 & -3.59093200 & -0.32567200 \\
\hline $\mathrm{H}$ & -2.58329900 & -3.17787300 & -0.30652500 \\
\hline 0 & -0.32045300 & -1.00165000 & 1.36634200 \\
\hline $\mathrm{H}$ & -0.51563200 & -1.31162700 & 0.44536300 \\
\hline
\end{tabular}

Zero-point correction=

0.690716 (Hartree/Particle) 


$\begin{array}{lc}\text { Thermal correction to Energy= } & 0.731103 \\ \text { Thermal correction to Enthalpy= } & 0.732047 \\ \text { Thermal correction to Gibbs Free Energy= } & 0.618203 \\ \text { Sum of electronic and zero-point Energies }= & -2047.195292 \\ \text { Sum of electronic and thermal Energies }= & -2047.154905 \\ \text { Sum of electronic and thermal Enthalpies }= & -2047.153961 \\ \text { Sum of electronic and thermal Free Energies }= & -2047.267805\end{array}$

$\mathrm{E}(\mathrm{RM} 062 \mathrm{X} / 6-311+\mathrm{G}(2 \mathrm{~d}, \mathrm{p}))=-2048.51009529$

$\begin{array}{cccc}\text { INT-2 } & & & \\ \mathrm{C} & 0.40119600 & -1.09308500 & -0.14529100 \\ \mathrm{~N} & 1.49218500 & -1.19950700 & 0.61719800 \\ \mathrm{~N} & -0.64584900 & -1.25989800 & 0.68171200 \\ \mathrm{C} & -0.13792100 & -1.40311600 & 1.93613900 \\ \mathrm{~N} & 1.16856500 & -1.39488600 & 1.91664100 \\ \mathrm{C} & 2.87796400 & -1.30508100 & 0.22683300 \\ \mathrm{C} & 5.53740400 & -1.53224000 & -0.49796100 \\ \mathrm{C} & 3.28425900 & -2.45974900 & -0.44959500 \\ \mathrm{C} & 3.75247400 & -0.27755400 & 0.59600600 \\ \mathrm{C} & 5.08433500 & -0.41662400 & 0.21097500 \\ \mathrm{C} & 4.62894700 & -2.54397600 & -0.81100200 \\ \mathrm{H} & 5.78759100 & 0.36911500 & 0.47657300 \\ \mathrm{H} & 4.97181700 & -3.42809900 & -1.34244300 \\ \mathrm{C} & 2.31997000 & -3.56073700 & -0.80404000 \\ \mathrm{H} & 1.71373000 & -3.29818200 & -1.68135200 \\ \mathrm{H} & 1.63863400 & -3.78592400 & 0.02272700 \\ \mathrm{H} & 2.86703200 & -4.47311400 & -1.05160400 \\ \mathrm{C} & 3.28305300 & 0.91113700 & 1.38578100 \\ \mathrm{H} & 4.06505400 & 1.67263100 & 1.42538500 \\ \mathrm{H} & 3.02910200 & 0.62353100 & 2.41169500 \\ \mathrm{H} & 2.38866900 & 1.35760300 & 0.94121400 \\ \mathrm{C} & 6.98096500 & -1.63185700 & -0.91346900 \\ \mathrm{H} & 7.64651500 & -1.37979200 & -0.08240400 \\ \mathrm{H} & 7.19738500 & -0.93201300 & -1.72819500 \\ \mathrm{H} & 7.22642100 & -2.63874700 & -1.25996700 \\ \mathrm{C} & -2.09321200 & -1.18594700 & 0.41452200 \\ \mathrm{H} & -2.25732200 & -0.34075400 & -0.25898600 \\ \mathrm{C} & -2.86878400 & -0.96116100 & 1.73561300 \\ \mathrm{H} & -2.83378800 & 0.08046000 & 2.06256900 \\ \mathrm{C} & -1.03702600 & -1.45025000 & 3.13255300 \\ \mathrm{H} & -1.01959100 & -0.45307000 & 3.58929100 \\ \mathrm{H} & -0.67581500 & -2.19085700 & 3.84849500 \\ \mathrm{C} & -2.33621500 & -1.81877900 & 2.74148400 \\ -4.26563000 & -1.48643300 & 1.40460000\end{array}$




\begin{tabular}{|c|c|c|c|}
\hline $\mathrm{H}$ & -4.85060100 & -0.69564000 & 0.92035500 \\
\hline $\mathrm{H}$ & -4.79155500 & -1.79711200 & 2.31107800 \\
\hline $\mathrm{C}$ & -3.97693000 & -2.61925600 & 0.44762200 \\
\hline $\mathrm{C}$ & -2.98041300 & -4.53012300 & -1.30286600 \\
\hline $\mathrm{C}$ & -4.76835600 & -3.71030700 & 0.11287900 \\
\hline $\mathrm{C}$ & -2.70276400 & -2.47349100 & -0.10872000 \\
\hline $\mathrm{C}$ & -2.18935300 & -3.42631000 & -0.97818200 \\
\hline $\mathrm{C}$ & -4.26174500 & -4.66598200 & -0.76954000 \\
\hline $\mathrm{H}$ & -5.75803700 & -3.82781100 & 0.54548600 \\
\hline $\mathrm{H}$ & -1.19233100 & -3.33007600 & -1.39658200 \\
\hline $\mathrm{H}$ & -4.86498900 & -5.53001300 & -1.03191900 \\
\hline $\mathrm{H}$ & -2.59228200 & -5.28861900 & -1.97573600 \\
\hline $\mathrm{C}$ & 0.26351000 & -0.80133100 & -1.62435500 \\
\hline 0 & -0.83206200 & -0.97800400 & -2.10257600 \\
\hline $\mathrm{C}$ & 1.42821400 & -0.25514900 & -2.42250000 \\
\hline $\mathrm{H}$ & 2.34986000 & -0.76707800 & -2.12718000 \\
\hline $\mathrm{C}$ & 1.18761900 & -0.47938500 & -3.91668000 \\
\hline $\mathrm{H}$ & 0.27811300 & 0.03009800 & -4.24230600 \\
\hline $\mathrm{H}$ & 1.09436700 & -1.54543300 & -4.14402900 \\
\hline $\mathrm{H}$ & 2.03386800 & -0.07851300 & -4.48052700 \\
\hline $\mathrm{C}$ & 1.57865500 & 1.23154900 & -2.13555500 \\
\hline $\mathrm{H}$ & 2.37358700 & 1.72340000 & -2.69648500 \\
\hline $\mathrm{C}$ & 0.78635100 & 1.94210400 & -1.37167000 \\
\hline $\mathrm{C}$ & -0.00136100 & 2.66366300 & -0.60652400 \\
\hline $\mathrm{C}$ & 0.44900500 & 2.99008800 & 0.78891600 \\
\hline $\mathrm{C}$ & 1.49109900 & 3.50139000 & 3.32330600 \\
\hline $\mathrm{C}$ & 1.39779900 & 3.99542700 & 0.97681900 \\
\hline $\mathrm{C}$ & -0.01238000 & 2.20945900 & 1.88443300 \\
\hline $\mathrm{C}$ & 0.54158000 & 2.50237100 & 3.15546400 \\
\hline $\mathrm{C}$ & 1.92645800 & 4.26610400 & 2.23733700 \\
\hline $\mathrm{H}$ & 1.73535800 & 4.56232900 & 0.11120600 \\
\hline $\mathrm{H}$ & 0.20258500 & 1.91055600 & 4.00233900 \\
\hline $\mathrm{H}$ & 2.66544100 & 5.05012500 & 2.36845600 \\
\hline $\mathrm{H}$ & 1.89573700 & 3.69130400 & 4.31490100 \\
\hline $\mathrm{C}$ & -1.24877800 & 3.25248800 & -1.19447300 \\
\hline $\mathrm{C}$ & -3.52946700 & 4.36843900 & -2.37597700 \\
\hline $\mathrm{C}$ & -2.53001700 & 2.86731300 & -0.74307100 \\
\hline $\mathrm{C}$ & -1.14238700 & 4.18335000 & -2.23232200 \\
\hline $\mathrm{C}$ & -2.26577600 & 4.74942600 & -2.82650700 \\
\hline $\mathrm{C}$ & -3.65786600 & 3.44030400 & -1.35148000 \\
\hline $\mathrm{H}$ & -0.14734600 & 4.46279900 & -2.56985000 \\
\hline $\mathrm{H}$ & -2.15607900 & 5.47434700 & -3.62665600 \\
\hline $\mathrm{H}$ & -4.63523300 & 3.12744600 & -0.99623400 \\
\hline $\mathrm{H}$ & -4.42176600 & 4.79594700 & -2.82505500 \\
\hline
\end{tabular}




$\begin{array}{llcc}0 & -0.88377300 & 1.25338700 & 1.71646300 \\ 0 & -2.74241800 & 1.95699700 & 0.21483500 \\ \mathrm{H} & -1.89683700 & 1.68758500 & 0.76711600\end{array}$

Zero-point correction $=$

0.663196 (Hartree/Particle)

Thermal correction to Energy=

0.701921

Thermal correction to Enthalpy=

0.702866

Thermal correction to Gibbs Free Energy=

0.593066

Sum of electronic and zero-point Energies=

$-1970.888549$

Sum of electronic and thermal Energies=

$-1970.849823$

Sum of electronic and thermal Enthalpies=

$-1970.848879$

Sum of electronic and thermal Free Energies=

$-1970.958679$

$\mathrm{E}(\mathrm{RM} 062 \mathrm{X} / 6-311+\mathrm{G}(2 \mathrm{~d}, \mathrm{p}))=-1972.13577394$

\section{INT-3B}

$\begin{array}{lrrr}\mathrm{C} & -1.22062800 & 0.76124500 & -0.17811100 \\ \mathrm{~N} & -2.42646100 & 1.09392000 & 0.25875500 \\ \mathrm{~N} & -0.37662300 & 1.68286600 & 0.31149600 \\ \mathrm{C} & -1.11495600 & 2.49658000 & 1.11780600 \\ \mathrm{~N} & -2.37979200 & 2.17827100 & 1.08123400 \\ \mathrm{C} & -3.68650700 & 0.46808100 & -0.03768300 \\ \mathrm{C} & -6.08644800 & -0.77846300 & -0.59532300 \\ \mathrm{C} & -4.23541600 & 0.63791200 & -1.30944400 \\ \mathrm{C} & -4.28816600 & -0.28633700 & 0.97653500 \\ \mathrm{C} & -5.49590700 & -0.90566600 & 0.66649300 \\ \mathrm{C} & -5.44681800 & -0.00744700 & -1.56588400 \\ \mathrm{H} & -5.98563400 & -1.51046700 & 1.42637900 \\ \mathrm{H} & -5.90035600 & 0.10335400 & -2.54767000 \\ \mathrm{C} & -3.55968100 & 1.47696000 & -2.36203500 \\ \mathrm{H} & -2.68173700 & 0.97279000 & -2.78282400 \\ \mathrm{H} & -3.22838100 & 2.43775900 & -1.95450100 \\ \mathrm{H} & -4.24971500 & 1.67457000 & -3.18532800 \\ \mathrm{C} & -3.63283800 & -0.43708400 & 2.32276100 \\ \mathrm{H} & -4.09737200 & -1.25464600 & 2.87883700 \\ \mathrm{H} & -3.74437000 & 0.48010600 & 2.91225900 \\ \mathrm{H} & -2.55704300 & -0.63446700 & 2.22269600 \\ \mathrm{C} & -7.40161800 & -1.45172300 & -0.88481100 \\ \mathrm{H} & -7.39287700 & -2.49195700 & -0.54556800 \\ \mathrm{H} & -7.62825700 & -1.43805900 & -1.95369000 \\ \mathrm{H} & -8.21839800 & -0.94421000 & -0.35966400 \\ \mathrm{C} & 1.09076500 & 1.66976200 & 0.29636000 \\ \mathrm{H} & 1.38918100 & 0.61692000 & 0.33491300 \\ \mathrm{C} & 1.61468700 & 2.42997300 & 1.53359100 \\ \mathrm{H} & 1.53450800 & 1.83745700 & 2.45361800 \\ \mathrm{C} & -0.44539500 & 3.48899400 & 2.02225600\end{array}$




\begin{tabular}{|c|c|c|c|}
\hline $\mathrm{H}$ & -0.54014100 & 3.11814700 & 3.05312400 \\
\hline $\mathrm{H}$ & -0.93592100 & 4.46204400 & 1.95529800 \\
\hline 0 & 0.90084300 & 3.65723700 & 1.65949800 \\
\hline $\mathrm{C}$ & 3.04194300 & 2.80011800 & 1.13028300 \\
\hline $\mathrm{H}$ & 3.71352000 & 1.95891700 & 1.33955200 \\
\hline $\mathrm{H}$ & 3.39444900 & 3.67191700 & 1.68706200 \\
\hline $\mathrm{C}$ & 1.75255400 & 2.41336100 & -0.84555300 \\
\hline $\mathrm{C}$ & 3.36505500 & 3.87151000 & -2.55738200 \\
\hline $\mathrm{C}$ & 2.90498200 & 3.03660200 & -0.35757700 \\
\hline $\mathrm{C}$ & 1.38681400 & 2.52140300 & -2.17968200 \\
\hline $\mathrm{C}$ & 2.20422300 & 3.26352000 & -3.03479000 \\
\hline $\mathrm{C}$ & 3.72375700 & 3.76274300 & -1.21227300 \\
\hline $\mathrm{H}$ & 0.48785900 & 2.04183800 & -2.55279300 \\
\hline $\mathrm{H}$ & 1.93270500 & 3.36650600 & -4.08102000 \\
\hline $\mathrm{H}$ & 4.61925700 & 4.25133200 & -0.83822700 \\
\hline $\mathrm{H}$ & 3.99038900 & 4.44421100 & -3.23584900 \\
\hline $\mathrm{C}$ & -0.76451300 & -0.44179900 & -0.97098300 \\
\hline 0 & 0.20187200 & -0.26784600 & -1.67938200 \\
\hline $\mathrm{C}$ & -1.50518900 & -1.76182800 & -0.89818100 \\
\hline $\mathrm{H}$ & -2.40443300 & -1.64675200 & -0.29089800 \\
\hline $\mathrm{C}$ & -1.89986800 & -2.15493800 & -2.32719900 \\
\hline $\mathrm{H}$ & -1.00627900 & -2.29850100 & -2.94100600 \\
\hline $\mathrm{H}$ & -2.52728100 & -1.38876100 & -2.79559200 \\
\hline $\mathrm{H}$ & -2.46532400 & -3.09068400 & -2.30724100 \\
\hline $\mathrm{C}$ & -0.63555700 & -2.86775700 & -0.22156800 \\
\hline $\mathrm{H}$ & -0.96168300 & -3.83247500 & -0.62805300 \\
\hline $\mathrm{H}$ & -0.82747400 & -2.85525500 & 0.84932600 \\
\hline $\mathrm{C}$ & 0.82806200 & -2.71636700 & -0.42203100 \\
\hline 0 & 1.29143500 & -3.02176700 & -1.67957700 \\
\hline $\mathrm{C}$ & 1.82424200 & -2.28894300 & 0.40519500 \\
\hline $\mathrm{C}$ & 3.02645300 & -2.30814000 & -0.41542600 \\
\hline $\mathrm{C}$ & 4.81418100 & -2.56821100 & -2.54283400 \\
\hline $\mathrm{C}$ & 2.62349400 & -2.76363000 & -1.67812400 \\
\hline $\mathrm{C}$ & 4.36939400 & -1.95381700 & -0.22737900 \\
\hline $\mathrm{C}$ & 5.24834300 & -2.08953300 & -1.29509200 \\
\hline $\mathrm{C}$ & 3.48416400 & -2.91321700 & -2.75743300 \\
\hline $\mathrm{H}$ & 4.71462600 & -1.57384600 & 0.72960500 \\
\hline $\mathrm{H}$ & 6.29167700 & -1.81769900 & -1.16621500 \\
\hline $\mathrm{H}$ & 3.12739000 & -3.27240900 & -3.71703200 \\
\hline $\mathrm{H}$ & 5.52719400 & -2.66403100 & -3.35589300 \\
\hline $\mathrm{C}$ & 1.74738600 & -1.89575500 & 1.82532700 \\
\hline $\mathrm{C}$ & 1.72507000 & -1.16823900 & 4.53643400 \\
\hline $\mathrm{C}$ & 2.76972800 & -2.29180600 & 2.69580000 \\
\hline $\mathrm{C}$ & 0.65248200 & -1.10110000 & 2.31472100 \\
\hline
\end{tabular}




$\begin{array}{lccc}\mathrm{C} & 0.69513500 & -0.76385900 & 3.70118900 \\ \mathrm{C} & 2.77977100 & -1.94259700 & 4.04394600 \\ \mathrm{H} & 3.57268900 & -2.91176700 & 2.30112400 \\ \mathrm{H} & -0.12700300 & -0.16184800 & 4.08337000 \\ \mathrm{H} & 3.58444800 & -2.27134500 & 4.69385600 \\ \mathrm{H} & 1.70673700 & -0.88112200 & 5.58587100 \\ 0 & -0.31238800 & -0.69587900 & 1.56200900\end{array}$

Zero-point correction= 0.667368 (Hartree/Particle)

Thermal correction to Energy= 0.705363

Thermal correction to Enthalpy=

0.706307

Thermal correction to Gibbs Free Energy=

0.596727

Sum of electronic and zero-point Energies=

$-1970.938310$

Sum of electronic and thermal Energies=

$-1970.900315$

Sum of electronic and thermal Enthalpies=

$-1970.899371$

Sum of electronic and thermal Free Energies=

$-1971.008951$

$\mathrm{E}(\mathrm{RM} 062 \mathrm{X} / 6-311+\mathrm{G}(2 \mathrm{~d}, \mathrm{p}))=-1972.17894993$

\section{INT-3}

$\begin{array}{lrrr}\mathrm{C} & -1.34948900 & 0.49226200 & 0.20078600 \\ \mathrm{~N} & -2.67908900 & 0.49899800 & 0.30402400 \\ \mathrm{~N} & -0.97013900 & 1.75790700 & 0.49326800 \\ \mathrm{C} & -2.10322700 & 2.45942500 & 0.80439600 \\ \mathrm{~N} & -3.16435400 & 1.72053600 & 0.67900800 \\ \mathrm{C} & -3.64265400 & -0.52568900 & -0.00829800 \\ \mathrm{C} & -5.52316900 & -2.45718000 & -0.62096500 \\ \mathrm{C} & -4.22539100 & -0.50067600 & -1.27538100 \\ \mathrm{C} & -3.99143500 & -1.44784000 & 0.98485200 \\ \mathrm{C} & -4.93483700 & -2.41367700 & 0.64838300 \\ \mathrm{C} & -5.16468100 & -1.49422000 & -1.56282200 \\ \mathrm{H} & -5.22073600 & -3.15097800 & 1.39524800 \\ \mathrm{H} & -5.62803200 & -1.50724100 & -2.54630400 \\ \mathrm{C} & -3.90437600 & 0.57732300 & -2.27605700 \\ \mathrm{H} & -2.85174500 & 0.87401200 & -2.24916400 \\ \mathrm{H} & -4.49987700 & 1.47473300 & -2.07258900 \\ \mathrm{H} & -4.13912900 & 0.24052300 & -3.28851100 \\ \mathrm{C} & -3.34772800 & -1.40153600 & 2.34315800 \\ \mathrm{H} & -2.27701500 & -1.62743500 & 2.28040800 \\ \mathrm{H} & -3.81796200 & -2.12481700 & 3.01295400 \\ \mathrm{H} & -3.44401100 & -0.40463200 & 2.78823500 \\ \mathrm{C} & -6.52764700 & -3.52980100 & -0.94891700 \\ \mathrm{H} & -7.33729800 & -3.54397900 & -0.21234900 \\ \mathrm{H} & -6.05804900 & -4.51914400 & -0.93442800 \\ \mathrm{H} & -6.96535900 & -3.37646300 & -1.93837000 \\ \mathrm{C} & 0.36799000 & 2.38453000 & 0.50500000\end{array}$




\begin{tabular}{|c|c|c|c|}
\hline $\mathrm{H}$ & 1.06257600 & 1.66282500 & 0.93814700 \\
\hline $\mathrm{C}$ & 0.32410500 & 3.68572300 & 1.33960300 \\
\hline $\mathrm{H}$ & 0.32616400 & 3.49388400 & 2.42037400 \\
\hline $\mathrm{C}$ & -2.02800000 & 3.85521600 & 1.34421300 \\
\hline $\mathrm{H}$ & -2.13370000 & 3.80792300 & 2.43827200 \\
\hline $\mathrm{H}$ & -2.84474400 & 4.45813100 & 0.94338600 \\
\hline 0 & -0.81556600 & 4.45789600 & 0.97673200 \\
\hline $\mathrm{C}$ & 1.53666900 & 4.46351500 & 0.82920000 \\
\hline $\mathrm{H}$ & 2.44230200 & 4.12904400 & 1.34860500 \\
\hline $\mathrm{H}$ & 1.41400800 & 5.53523200 & 1.00439100 \\
\hline $\mathrm{C}$ & 1.56901000 & 4.07806600 & -0.63236400 \\
\hline $\mathrm{C}$ & 1.33855700 & 3.00316700 & -3.17953200 \\
\hline $\mathrm{C}$ & 2.16359300 & 4.73752000 & -1.70051000 \\
\hline $\mathrm{C}$ & 0.87279500 & 2.88332900 & -0.83702300 \\
\hline $\mathrm{C}$ & 0.75453700 & 2.33007000 & -2.10464900 \\
\hline $\mathrm{C}$ & 2.03943600 & 4.19282800 & -2.97970300 \\
\hline $\mathrm{H}$ & 2.70397600 & 5.66741900 & -1.54521500 \\
\hline $\mathrm{H}$ & 0.24435700 & 1.38342000 & -2.24531900 \\
\hline $\mathrm{H}$ & 2.49039900 & 4.70082200 & -3.82715600 \\
\hline $\mathrm{H}$ & 1.25276500 & 2.59073000 & -4.18034400 \\
\hline $\mathrm{C}$ & -0.28648300 & -0.65233300 & 0.01212300 \\
\hline 0 & 0.76487200 & -0.13513800 & -0.52811200 \\
\hline $\mathrm{C}$ & -0.86485300 & -1.94665100 & -0.64181900 \\
\hline $\mathrm{H}$ & -1.87090600 & -2.12923400 & -0.25494600 \\
\hline $\mathrm{C}$ & -0.93486000 & -1.77798500 & -2.15691000 \\
\hline $\mathrm{H}$ & 0.07197000 & -1.63743400 & -2.56040800 \\
\hline $\mathrm{H}$ & -1.54287900 & -0.90799600 & -2.43283000 \\
\hline $\mathrm{H}$ & -1.38155500 & -2.66085500 & -2.62422800 \\
\hline $\mathrm{C}$ & -0.04544700 & -3.15000200 & -0.19881800 \\
\hline $\mathrm{H}$ & -0.55807000 & -4.09988000 & -0.06240700 \\
\hline $\mathrm{C}$ & 1.19706900 & -2.96406600 & 0.15445800 \\
\hline $\mathrm{C}$ & 2.30575600 & -2.38286800 & 0.55786200 \\
\hline $\mathrm{C}$ & 2.25098200 & -1.48832100 & 1.75072300 \\
\hline $\mathrm{C}$ & 2.20516400 & 0.27458900 & 3.95474000 \\
\hline $\mathrm{C}$ & 3.40188000 & -1.22404100 & 2.50231700 \\
\hline $\mathrm{C}$ & 1.05642300 & -0.81518600 & 2.11040300 \\
\hline $\mathrm{C}$ & 1.05244200 & 0.04874500 & 3.20896000 \\
\hline $\mathrm{C}$ & 3.39004600 & -0.36076700 & 3.59395600 \\
\hline $\mathrm{H}$ & 4.33139700 & -1.70728400 & 2.21711800 \\
\hline $\mathrm{H}$ & 0.11573200 & 0.54081300 & 3.45864600 \\
\hline $\mathrm{H}$ & 4.30278500 & -0.18659700 & 4.15516100 \\
\hline $\mathrm{H}$ & 2.17715700 & 0.95009600 & 4.80471100 \\
\hline $\mathrm{C}$ & 3.55046800 & -2.48951900 & -0.26645800 \\
\hline $\mathrm{C}$ & 5.84898700 & -2.75889100 & -1.84131400 \\
\hline
\end{tabular}




\begin{tabular}{|c|c|c|c|}
\hline $\mathrm{C}$ & 3.63496100 & -1.81897700 & -1.50377500 \\
\hline $\mathrm{C}$ & 4.61921300 & -3.28928700 & 0.15017300 \\
\hline $\mathrm{C}$ & 5.76978500 & -3.42834900 & -0.61967400 \\
\hline $\mathrm{C}$ & 4.79599000 & -1.96663800 & -2.27798000 \\
\hline $\mathrm{H}$ & 4.53008200 & -3.82166300 & 1.09431200 \\
\hline $\mathrm{H}$ & 6.58622900 & -4.05567000 & -0.27668600 \\
\hline $\mathrm{H}$ & 4.84342100 & -1.44195500 & -3.22748700 \\
\hline $\mathrm{H}$ & 6.73690800 & -2.85584200 & -2.45995400 \\
\hline 0 & -0.14348500 & -0.99805900 & 1.48826800 \\
\hline 0 & 2.65455900 & -1.05500300 & -2.00285600 \\
\hline $\mathrm{H}$ & 1.92138500 & -0.84099600 & -1.34164500 \\
\hline \multicolumn{3}{|c|}{ Zero-point correction= } & 0.663906 (Hartree/Particle) \\
\hline \multicolumn{3}{|c|}{ Thermal correction to Energy= } & 0.702186 \\
\hline \multicolumn{3}{|c|}{ Thermal correction to Enthalpy= } & 0.703130 \\
\hline \multicolumn{3}{|c|}{ Thermal correction to Gibbs Free Energy= } & 0.593193 \\
\hline \multicolumn{3}{|c|}{ Sum of electronic and zero-point Energies $=$} & -1970.882256 \\
\hline \multicolumn{3}{|c|}{ Sum of electronic and thermal Energies= } & -1970.843975 \\
\hline \multicolumn{3}{|c|}{ Sum of electronic and thermal Enthalpies= } & -1970.843031 \\
\hline \multicolumn{3}{|c|}{ Sum of electronic and thermal Free Energies= } & -1970.952969 \\
\hline \multicolumn{4}{|c|}{$\mathrm{E}(\mathrm{RM} 062 \mathrm{X} / 6-311+\mathrm{G}(2 \mathrm{~d}, \mathrm{p}))=-1972.12465396$} \\
\hline \multicolumn{4}{|c|}{ INT-4B } \\
\hline $\mathrm{C}$ & -1.35372100 & 0.58175700 & 0.12114200 \\
\hline $\mathrm{N}$ & -2.68625900 & 0.56353900 & 0.17769500 \\
\hline $\mathrm{N}$ & -1.01965800 & 1.87804700 & 0.31625500 \\
\hline $\mathrm{C}$ & -2.17813400 & 2.57348900 & 0.52779000 \\
\hline $\mathrm{N}$ & -3.21686600 & 1.79975300 & 0.42999100 \\
\hline $\mathrm{C}$ & -3.61103600 & -0.51945700 & -0.03360000 \\
\hline $\mathrm{C}$ & -5.41417900 & -2.57608500 & -0.43994500 \\
\hline $\mathrm{C}$ & -4.17854100 & -0.65825100 & -1.29989700 \\
\hline $\mathrm{C}$ & -3.93958400 & -1.33713400 & 1.05329200 \\
\hline $\mathrm{C}$ & -4.84327000 & -2.36927800 & 0.82104100 \\
\hline $\mathrm{C}$ & -5.07651200 & -1.71335000 & -1.48153800 \\
\hline $\mathrm{H}$ & -5.10924400 & -3.02951500 & 1.64361600 \\
\hline $\mathrm{H}$ & -5.52634900 & -1.85490700 & -2.46130600 \\
\hline $\mathrm{C}$ & -3.88296200 & 0.31615800 & -2.40869600 \\
\hline $\mathrm{H}$ & -2.82402400 & 0.58808000 & -2.45248600 \\
\hline $\mathrm{H}$ & -4.44884400 & 1.24306900 & -2.25986400 \\
\hline $\mathrm{H}$ & -4.16981800 & -0.10446500 & -3.37518500 \\
\hline $\mathrm{C}$ & -3.30892500 & -1.11815000 & 2.40104100 \\
\hline $\mathrm{H}$ & -2.22469300 & -1.27610600 & 2.36037300 \\
\hline $\mathrm{H}$ & -3.73486600 & -1.80336000 & 3.13725300 \\
\hline $\mathrm{H}$ & -3.47335300 & -0.09204800 & 2.74923500 \\
\hline $\mathrm{C}$ & -6.38734400 & -3.70586100 & -0.65137800 \\
\hline
\end{tabular}




\begin{tabular}{|c|c|c|c|}
\hline $\mathrm{H}$ & -5.94705600 & -4.66261200 & -0.35279400 \\
\hline $\mathrm{H}$ & -6.68904600 & -3.77905700 & -1.69911200 \\
\hline $\mathrm{H}$ & -7.28894700 & -3.56002300 & -0.04684400 \\
\hline $\mathrm{C}$ & 0.30462300 & 2.52627100 & 0.34137100 \\
\hline $\mathrm{H}$ & 0.99276500 & 1.84222000 & 0.84222200 \\
\hline $\mathrm{C}$ & 0.20271700 & 3.87435400 & 1.09245400 \\
\hline $\mathrm{H}$ & 0.15376800 & 3.74913100 & 2.18179700 \\
\hline $\mathrm{C}$ & -2.15447800 & 4.00922300 & 0.95597900 \\
\hline $\mathrm{H}$ & -2.33621800 & 4.04763700 & 2.04019800 \\
\hline $\mathrm{H}$ & -2.94749100 & 4.56559100 & 0.45296800 \\
\hline 0 & -0.92751200 & 4.60409900 & 0.62483700 \\
\hline $\mathrm{C}$ & 1.42493900 & 4.64276900 & 0.59129500 \\
\hline $\mathrm{H}$ & 2.31234100 & 4.35461100 & 1.16697800 \\
\hline $\mathrm{H}$ & 1.27866000 & 5.72049200 & 0.69806700 \\
\hline $\mathrm{C}$ & 1.52453500 & 4.17440700 & -0.84310900 \\
\hline $\mathrm{C}$ & 1.41267700 & 2.95215400 & -3.33077600 \\
\hline $\mathrm{C}$ & 2.14797100 & 4.78455700 & -1.92414700 \\
\hline $\mathrm{C}$ & 0.85742200 & 2.95680500 & -1.00532300 \\
\hline $\mathrm{C}$ & 0.79896800 & 2.32942600 & -2.24194700 \\
\hline $\mathrm{C}$ & 2.08342600 & 4.16554000 & -3.17371900 \\
\hline $\mathrm{H}$ & 2.66506800 & 5.73256200 & -1.80239700 \\
\hline $\mathrm{H}$ & 0.31661900 & 1.36315500 & -2.34111200 \\
\hline $\mathrm{H}$ & 2.55688600 & 4.63426100 & -4.03154500 \\
\hline $\mathrm{H}$ & 1.37389700 & 2.48179700 & -4.30882500 \\
\hline $\mathrm{C}$ & -0.22390500 & -0.53436900 & 0.01356800 \\
\hline 0 & 0.79510100 & -0.06749800 & -0.57140400 \\
\hline $\mathrm{C}$ & -0.75740200 & -1.92329400 & -0.42704900 \\
\hline $\mathrm{C}$ & 0.09796400 & -3.04382600 & 0.20508400 \\
\hline $\mathrm{H}$ & -0.11330000 & -3.09632900 & 1.27474900 \\
\hline $\mathrm{C}$ & 1.56022100 & -2.85057700 & 0.01046200 \\
\hline $\mathrm{C}$ & 2.48495600 & -2.08928100 & 0.65753600 \\
\hline 0 & 2.09300700 & -3.49446100 & -1.07506000 \\
\hline $\mathrm{C}$ & 3.71357100 & -2.24513800 & -0.10873400 \\
\hline $\mathrm{C}$ & 5.58029500 & -3.01974900 & -2.04903900 \\
\hline $\mathrm{C}$ & 5.01104500 & -1.71027800 & -0.07248700 \\
\hline $\mathrm{C}$ & 3.39797400 & -3.12764000 & -1.15117800 \\
\hline $\mathrm{C}$ & 4.29562700 & -3.54509200 & -2.12388200 \\
\hline $\mathrm{C}$ & 5.92632900 & -2.10608300 & -1.04028800 \\
\hline $\mathrm{H}$ & 5.29891400 & -0.98461500 & 0.68072400 \\
\hline $\mathrm{H}$ & 3.99571400 & -4.23578200 & -2.90494200 \\
\hline $\mathrm{H}$ & 6.93101200 & -1.69472200 & -1.01973300 \\
\hline $\mathrm{H}$ & 6.32092000 & -3.31028700 & -2.78756400 \\
\hline $\mathrm{C}$ & 2.30903600 & -1.21803300 & 1.83548300 \\
\hline $\mathrm{C}$ & 2.20698100 & 0.47468300 & 4.10649000 \\
\hline
\end{tabular}




\begin{tabular}{|c|c|c|c|}
\hline $\mathrm{C}$ & 1.11056300 & -0.54291400 & 2.17808900 \\
\hline $\mathrm{C}$ & 3.41077800 & -1.04421000 & 2.69021200 \\
\hline $\mathrm{C}$ & 3.37790200 & -0.21629900 & 3.80632700 \\
\hline $\mathrm{C}$ & 1.09261100 & 0.30496600 & 3.29523500 \\
\hline $\mathrm{H}$ & 4.31650200 & -1.60411500 & 2.47980200 \\
\hline $\mathrm{H}$ & 4.25676800 & -0.12058100 & 4.43619500 \\
\hline $\mathrm{H}$ & 0.15689400 & 0.81273200 & 3.51482100 \\
\hline $\mathrm{H}$ & 2.15613800 & 1.13224400 & 4.96950700 \\
\hline 0 & -0.08296000 & -0.71673400 & 1.56524000 \\
\hline $\mathrm{H}$ & -1.76961200 & -2.07823100 & 0.04519700 \\
\hline $\mathrm{C}$ & -0.77533700 & -1.99417200 & 1.94843000 \\
\hline $\mathrm{H}$ & -1.16031500 & -2.95975900 & 2.29251600 \\
\hline $\mathrm{H}$ & 0.23473700 & -1.85069200 & 2.34219400 \\
\hline $\mathrm{H}$ & -1.41344600 & -1.20937300 & 2.37242500 \\
\hline $\mathrm{H}$ & -0.18940400 & -4.00184200 & 0.24132900 \\
\hline \multicolumn{3}{|c|}{ Zero-point correction= } & 0.667530 (Hartree/Particle) \\
\hline \multicolumn{3}{|c|}{ Thermal correction to Energy= } & 0.704999 \\
\hline \multicolumn{3}{|c|}{ Thermal correction to Enthalpy= } & 0.705943 \\
\hline \multicolumn{3}{|c|}{ Thermal correction to Gibbs Free Energy= } & 0.598045 \\
\hline \multicolumn{3}{|c|}{ Sum of electronic and zero-point Energies= } & -1970.934816 \\
\hline \multicolumn{3}{|c|}{ Sum of electronic and thermal Energies= } & -1970.897347 \\
\hline \multicolumn{3}{|c|}{ Sum of electronic and thermal Enthalpies= } & -1970.896403 \\
\hline \multicolumn{3}{|c|}{ Sum of electronic and thermal Free Energies= } & -1971.004301 \\
\hline \multicolumn{4}{|c|}{$\mathrm{E}(\mathrm{RM} 062 \mathrm{X} / 6-311+\mathrm{G}(2 \mathrm{~d}, \mathrm{p}))=-1972.17138187$} \\
\hline \multicolumn{4}{|c|}{ INT-4 } \\
\hline $\mathrm{C}$ & 1.92927200 & -1.24824200 & 0.60127700 \\
\hline 0 & 1.31844900 & -1.08042700 & 1.62594500 \\
\hline $\mathrm{C}$ & 1.98758000 & -2.53108100 & 0.20577000 \\
\hline $\mathrm{H}$ & 3.02439900 & -2.70258900 & 0.51717200 \\
\hline $\mathrm{C}$ & 1.18361700 & -2.18253300 & 1.47179500 \\
\hline $\mathrm{H}$ & 1.41440600 & -2.69922600 & 2.39908000 \\
\hline $\mathrm{C}$ & 0.33128200 & -1.19639100 & 1.35986200 \\
\hline $\mathrm{C}$ & -0.23205500 & -0.12131000 & 0.83582900 \\
\hline $\mathrm{C}$ & -1.65981600 & -0.12283200 & 0.41349200 \\
\hline $\mathrm{C}$ & -4.35229200 & -0.05959700 & 0.34531200 \\
\hline $\mathrm{C}$ & -2.66924600 & -0.45508800 & 1.32281200 \\
\hline $\mathrm{C}$ & -2.01638400 & 0.21135700 & 0.90740800 \\
\hline $\mathrm{C}$ & -3.36405400 & 0.25488800 & 1.27055800 \\
\hline $\mathrm{C}$ & -4.01004400 & -0.42861600 & 0.95514900 \\
\hline $\mathrm{H}$ & -2.38114200 & -0.71634600 & 2.33726000 \\
\hline $\mathrm{H}$ & -3.61197300 & 0.51910700 & 2.29377000 \\
\hline $\mathrm{H}$ & -4.77866900 & -0.68230100 & 1.67777000 \\
\hline $\mathrm{H}$ & -5.39513100 & -0.02471400 & 0.64632100 \\
\hline
\end{tabular}




\begin{tabular}{|c|c|c|c|}
\hline $\mathrm{C}$ & 0.59419400 & 1.09789600 & -0.57797400 \\
\hline $\mathrm{C}$ & 2.10665100 & 3.38919300 & 0.02973200 \\
\hline $\mathrm{C}$ & 1.92656600 & 1.00098800 & -0.13405800 \\
\hline $\mathrm{C}$ & 0.03696500 & 2.37711900 & -0.67717000 \\
\hline $\mathrm{C}$ & 0.78250900 & 3.51410000 & -0.38614600 \\
\hline $\mathrm{C}$ & 2.67950600 & 2.12789700 & 0.16322100 \\
\hline $\mathrm{H}$ & -0.99905000 & 2.46854700 & -0.99143800 \\
\hline $\mathrm{H}$ & 0.32901400 & 4.49538300 & -0.48014900 \\
\hline $\mathrm{H}$ & 3.70313200 & 2.00125400 & 0.49983900 \\
\hline $\mathrm{H}$ & 2.69382700 & 4.27172800 & 0.26277000 \\
\hline 0 & -1.11084700 & 0.50445900 & 1.87101300 \\
\hline $\mathrm{H}$ & -0.22219300 & 0.18116700 & 1.63510000 \\
\hline 0 & 2.55976000 & -0.23175200 & -0.05519800 \\
\hline $\mathrm{C}$ & 1.44154800 & -3.72434200 & 0.56611600 \\
\hline $\mathrm{H}$ & 0.41208900 & -3.53166800 & 0.87916600 \\
\hline $\mathrm{H}$ & 2.04506800 & -3.92288000 & 1.45632000 \\
\hline $\mathrm{H}$ & 1.45253800 & -4.61614400 & -0.06589100 \\
\hline \multicolumn{3}{|c|}{ Zero-point correction $=$} & 0.277409 (Hartree/Particle) \\
\hline \multicolumn{3}{|c|}{ Thermal correction to Energy= } & 0.294088 \\
\hline \multicolumn{3}{|c|}{ Thermal correction to Enthalpy= } & 0.295033 \\
\hline \multicolumn{3}{|c|}{ Thermal correction to Gibbs Free Energy= } & 0.233568 \\
\hline \multicolumn{3}{|c|}{ Sum of electronic and zero-point Energies= } & -919.335910 \\
\hline \multicolumn{3}{|c|}{ Sum of electronic and thermal Energies= } & -919.319231 \\
\hline \multicolumn{3}{|c|}{ Sum of electronic and thermal Enthalpies= } & -919.318287 \\
\hline \multicolumn{3}{|c|}{ Sum of electronic and thermal Free Energies= } & -919.379751 \\
\hline
\end{tabular}

$\mathrm{E}(\mathrm{RM} 062 \mathrm{X} / 6-311+\mathrm{G}(2 \mathrm{~d}, \mathrm{p}))=-919.895519385$

$\mathrm{NHC}$

$\begin{array}{lrrr}\mathrm{N} & -0.77404600 & -1.26826300 & 1.06690000 \\ \mathrm{C} & 0.48944700 & -1.42297500 & 0.81898300 \\ \mathrm{~N} & 0.83759000 & -0.76760000 & -0.33246200 \\ \mathrm{C} & -0.24894800 & -0.14040500 & -0.88516900 \\ \mathrm{~N} & -1.19472500 & -0.48366800 & 0.01121500 \\ \mathrm{C} & 2.20126600 & -0.73317400 & -0.84216800 \\ \mathrm{H} & 2.16598700 & -0.97719300 & -1.90913800 \\ \mathrm{C} & -2.56946600 & -0.08276800 & -0.02380600 \\ \mathrm{C} & -5.23798300 & 0.69380600 & -0.06472500 \\ \mathrm{C} & -3.45468100 & -0.76412800 & -0.86278800 \\ \mathrm{C} & -2.97787700 & 0.96864200 & 0.80305200 \\ \mathrm{C} & -4.32026000 & 1.34341900 & 0.76408700 \\ \mathrm{C} & -4.78837200 & -0.35329300 & -0.87073500 \\ \mathrm{H} & -4.65638400 & 2.16334300 & 1.39523200 \\ \mathrm{H} & -5.49289900 & -0.86880200 & -1.51979900 \\ \mathrm{C} & -1.98985300 & 1.66448600 & 1.69966400\end{array}$




\begin{tabular}{|c|c|c|c|}
\hline $\mathrm{H}$ & -2.45188900 & 2.52363100 & 2.19155700 \\
\hline $\mathrm{H}$ & -1.12105900 & 2.01439800 & 1.13155600 \\
\hline $\mathrm{H}$ & -1.61496600 & 0.98488000 & 2.47257400 \\
\hline $\mathrm{C}$ & -2.97983900 & -1.89974300 & .72892800 \\
\hline $\mathrm{H}$ & -2.32187000 & -1.53945000 & .52639000 \\
\hline $\mathrm{H}$ & -3.82786100 & -2.41122800 & 2.19069500 \\
\hline $\mathrm{H}$ & -2.41118000 & -2.63021500 & 1.14359000 \\
\hline $\mathrm{C}$ & -6.69034500 & 1.09503200 & 0.06285500 \\
\hline $\mathrm{H}$ & -7.16925100 & 0.85872000 & 1.01704100 \\
\hline $\mathrm{H}$ & -6.80550900 & 2.16632700 & 0.12528600 \\
\hline $\mathrm{H}$ & -7.23904100 & 0.56308200 & 0.72309300 \\
\hline $\mathrm{C}$ & 3.09679000 & -1.75575800 & 0.09985700 \\
\hline $\mathrm{H}$ & 2.97963900 & -2.77293600 & 0.49551900 \\
\hline $\mathrm{C}$ & 1.50429600 & -2.22850000 & 1.57094600 \\
\hline $\mathrm{H}$ & 1.41851300 & -3.28840900 & 1.28969700 \\
\hline $\mathrm{H}$ & 1.34210200 & -2.14200600 & 2.64675800 \\
\hline 0 & 2.79889400 & -1.74138600 & 1.28991400 \\
\hline $\mathrm{C}$ & 4.51031800 & -1.18727900 & 0.25556300 \\
\hline $\mathrm{H}$ & 4.95821800 & -1.54755800 & 1.18913500 \\
\hline $\mathrm{H}$ & 5.14932600 & -1.50457600 & 0.57273100 \\
\hline $\mathrm{C}$ & 4.27153800 & 0.30516900 & 0.30759100 \\
\hline $\mathrm{C}$ & 3.35929400 & 2.92352600 & 0.49215900 \\
\hline $\mathrm{C}$ & 5.16246800 & 1.34974500 & 0.09331900 \\
\hline $\mathrm{C}$ & 2.93702700 & 0.57293100 & 0.62043700 \\
\hline $\mathrm{C}$ & 2.46460200 & 1.87468500 & 0.71456200 \\
\hline $\mathrm{C}$ & 4.69597800 & 2.66267200 & 0.18786600 \\
\hline $\mathrm{H}$ & 6.20106400 & 1.15042600 & 0.15651600 \\
\hline $\mathrm{H}$ & 1.42188600 & 2.06949200 & 0.94933500 \\
\hline $\mathrm{H}$ & 5.37883400 & 3.48920300 & 0.01449400 \\
\hline $\mathrm{H}$ & 3.01105600 & 3.95015200 & 0.55398700 \\
\hline \multicolumn{3}{|c|}{ Zero-point correction $=$} & 0.384742 (Hartree/Particle) \\
\hline \multicolumn{3}{|c|}{ Thermal correction to Energy= } & 0.405605 \\
\hline \multicolumn{3}{|c|}{ Thermal correction to Enthalpy= } & 0.406549 \\
\hline \multicolumn{3}{|c|}{ Thermal correction to Gibbs Free Energy= } & 0.334299 \\
\hline \multicolumn{3}{|c|}{ Sum of electronic and zero-point Energies= } & -1051.533723 \\
\hline \multicolumn{3}{|c|}{ Sum of electronic and thermal Energies= } & -1051.512860 \\
\hline \multicolumn{3}{|c|}{ Sum of electronic and thermal Enthalpies= } & -1051.511915 \\
\hline \multicolumn{3}{|c|}{ Sum of electronic and thermal Free Energies= } & -1051.584166 \\
\hline \multicolumn{4}{|c|}{$\mathrm{E}(\mathrm{RM} 062 \mathrm{X} / 6-311+\mathrm{G}(2 \mathrm{~d}, \mathrm{p}))=-1052.22310475$} \\
\hline \multicolumn{4}{|c|}{$\mathrm{o}-\mathrm{QM}$} \\
\hline $\mathrm{H}$ & -0.19140100 & 4.50545100 & 0.62425800 \\
\hline $\mathrm{C}$ & -0.09697700 & 3.44818000 & 0.47971500 \\
\hline $\mathrm{C}$ & 0.01435800 & 2.25506900 & 0.32297600 \\
\hline
\end{tabular}




$\begin{array}{lccc}\mathrm{C} & 0.08708500 & 0.83405700 & 0.13102500 \\ \mathrm{C} & 1.34191800 & 0.25096800 & 0.13044700 \\ \mathrm{C} & 1.56698400 & -1.15122500 & -0.30518900 \\ \mathrm{C} & 2.92241500 & -1.51448500 & -0.69296800 \\ \mathrm{H} & 3.05507700 & -2.51445000 & -1.09265500 \\ \mathrm{C} & 3.96144800 & -0.67578900 & -0.47996800 \\ \mathrm{H} & 4.97136500 & -0.98908500 & -0.72975700 \\ \mathrm{C} & 3.76248600 & 0.63331900 & 0.09573200 \\ \mathrm{H} & 4.62378000 & 1.26463500 & 0.28569500 \\ \mathrm{C} & 2.51636300 & 1.07140800 & 0.37915400 \\ \mathrm{H} & 2.36767200 & 2.06421200 & 0.78961000 \\ \mathrm{O} & 0.65880000 & -1.99591700 & -0.35506100 \\ \mathrm{C} & -1.25318300 & 0.24159500 & -0.09144600 \\ \mathrm{C} & -3.94566100 & -0.52446400 & -0.35322800 \\ \mathrm{C} & -2.12942800 & 0.92601200 & -0.95914400 \\ \mathrm{C} & -1.76467500 & -0.84532500 & 0.65158400 \\ \mathrm{C} & -3.11240600 & -1.20193700 & 0.52077300 \\ \mathrm{C} & -3.44741500 & 0.53740400 & -1.11561900 \\ \mathrm{H} & -1.74755400 & 1.77312200 & -1.52121000 \\ \mathrm{H} & -3.47267000 & -2.03250500 & 1.11903300 \\ \mathrm{H} & -4.09091400 & 1.06655800 & -1.81055100 \\ \mathrm{H} & -4.98523900 & -0.82376200 & -0.44781400 \\ \mathrm{O} & -1.01467200 & -1.52910200 & 1.53968000 \\ \mathrm{H} & -0.22302000 & -1.82274500 & 1.02338000\end{array}$

Zero-point correction=

Thermal correction to Energy=

0.206157 (Hartree/Particle)

Thermal correction to Enthalpy=

0.219805

Thermal correction to Gibbs Free Energy=

0.220750

Sum of electronic and zero-point Energies=

0.165483

Sum of electronic and thermal Energies=

$-727.468637$

$-727.454989$

$-727.454044$

$-727.509311$

Sum of electronic and thermal Free Energies=

$\mathrm{E}(\mathrm{RM} 062 \mathrm{X} / 6-311+\mathrm{G}(2 \mathrm{~d}, \mathrm{p}))=-727.900897008$

Trimethylamine

$\begin{array}{crrc}\mathrm{N} & -0.39718400 & 0.00043000 & 0.00000000 \\ \mathrm{C} & 0.06403600 & 1.37731500 & 0.00000000 \\ \mathrm{H} & -0.31273900 & 1.89545500 & 0.88757400 \\ \mathrm{H} & -0.31273900 & 1.89545500 & -0.88757400 \\ \mathrm{H} & 1.16799000 & 1.45413500 & 0.00000000 \\ \mathrm{C} & 0.06403600 & -0.68870100 & 1.19195000 \\ \mathrm{H} & -0.31438000 & -0.18149300 & 2.08506300 \\ \mathrm{H} & 1.16801700 & -0.72520700 & 1.25908100 \\ \mathrm{H} & -0.31106900 & -1.71706500 & 1.19461500\end{array}$




$\begin{array}{ccccc}\text { C } & 0.06403600 & -0.68870100 & -1.19195000 & \\ \mathrm{H} & -0.31106900 & -1.71706500 & -1.19461500 & \\ \mathrm{H} & 1.16801700 & -0.72520700 & -1.25908100 & \\ \mathrm{H} & -0.31438000 & -0.18149300 & -2.08506300 & \\ \text { Zero-point correction= } & 0.122264 \text { (Hartree/Particle) } \\ \text { Thermal correction to Energy= } & 0.127553 \\ \text { Thermal correction to Enthalpy= } & 0.128498 \\ \text { Thermal correction to Gibbs Free Energy= } & 0.095120 \\ \text { Sum of electronic and zero-point Energies }= & -174.257255 \\ \text { Sum of electronic and thermal Energies }= & -174.251966 \\ \text { Sum of electronic and thermal Enthalpies }= & -174.251022 \\ \text { Sum of electronic and thermal Free Energies }= & -174.284399 \\ \text { E(RM062X/6-311+G(2d, p)) = -174.437247217 } & \end{array}$

TS1

$\begin{array}{lrrr}\mathrm{N} & 2.64620200 & 1.75833100 & 1.49050300 \\ \mathrm{C} & 3.00716500 & 0.51076000 & 1.56372400 \\ \mathrm{~N} & 2.38811600 & -0.24185700 & 0.60812200 \\ \mathrm{C} & 1.58476500 & 0.59168000 & -0.09887000 \\ \mathrm{~N} & 1.76554000 & 1.79792300 & 0.45370400 \\ \mathrm{C} & 1.13031600 & 3.05527200 & 0.16108800 \\ \mathrm{C} & -0.03950700 & 5.50325900 & -0.34929000 \\ \mathrm{C} & 0.00125500 & 3.40772700 & 0.89769500 \\ \mathrm{C} & 1.72179700 & 3.88292600 & -0.80063800 \\ \mathrm{C} & 1.10908700 & 5.10630100 & -1.04686200 \\ \mathrm{C} & -0.57320400 & 4.65087000 & 0.61540600 \\ \mathrm{H} & 1.53517500 & 5.76927200 & -1.79646600 \\ \mathrm{H} & -1.46317400 & 4.95055300 & 1.16342700 \\ \mathrm{C} & 2.95373300 & 3.43646600 & -1.54036700 \\ \mathrm{H} & 3.29052100 & 4.21280600 & -2.23047100 \\ \mathrm{H} & 2.75729800 & 2.52548200 & -2.11978300 \\ \mathrm{H} & 3.77126400 & 3.21213100 & -0.84633600 \\ \mathrm{C} & -0.56653600 & 2.49543000 & 1.95047700 \\ \mathrm{H} & -0.06937700 & 2.66434400 & 2.91342900 \\ \mathrm{H} & -0.43486700 & 1.44388000 & 1.68327600 \\ \mathrm{H} & -1.63562400 & 2.68031700 & 2.08366900 \\ \mathrm{C} & -0.67992600 & 6.83266400 & -0.64858300 \\ \mathrm{H} & 0.03662000 & 7.65014400 & -0.51798200 \\ \mathrm{H} & -1.53426900 & 7.01757000 & 0.00706000 \\ \mathrm{H} & -1.03129300 & 6.87096600 & -1.68512400 \\ \mathrm{C} & 0.70549800 & 0.14209600 & -1.21728100 \\ \mathrm{C} & 2.61948000 & -1.69030000 & 0.46786000 \\ \mathrm{H} & 1.64386700 & -2.16913700 & 0.39000000 \\ \mathrm{C} & 3.37937400 & -2.22780600 & 1.70325700\end{array}$




\begin{tabular}{|c|c|c|c|}
\hline $\mathrm{H}$ & 2.70929400 & -2.39267200 & 2.55618600 \\
\hline $\mathrm{C}$ & 3.92021000 & -0.11842000 & 2.57131200 \\
\hline $\mathrm{H}$ & 3.36495200 & -0.27427700 & 3.50748100 \\
\hline $\mathrm{H}$ & 4.76454900 & 0.54369400 & 2.77118100 \\
\hline 0 & 4.41993900 & -1.33089400 & 2.06695200 \\
\hline $\mathrm{C}$ & 4.05272100 & -3.49597100 & 1.17522300 \\
\hline $\mathrm{H}$ & 4.94990800 & -3.72765200 & 1.75467400 \\
\hline $\mathrm{H}$ & 3.36432800 & -4.34518100 & 1.25408000 \\
\hline $\mathrm{C}$ & 3.52180300 & -2.08749700 & -0.68275000 \\
\hline $\mathrm{C}$ & 5.32340500 & -3.22236500 & -2.45073100 \\
\hline $\mathrm{C}$ & 3.60628600 & -1.57899600 & -1.97208400 \\
\hline $\mathrm{C}$ & 4.32930500 & -3.15064000 & -0.27012400 \\
\hline $\mathrm{C}$ & 5.23181500 & -3.72938000 & -1.15348200 \\
\hline $\mathrm{C}$ & 4.52253300 & -2.15396400 & -2.85469900 \\
\hline $\mathrm{H}$ & 2.98451700 & -0.74893800 & -2.29461000 \\
\hline $\mathrm{H}$ & 5.86521900 & -4.55328000 & -0.83648000 \\
\hline $\mathrm{H}$ & 4.61033300 & -1.76592000 & -3.86481000 \\
\hline $\mathrm{H}$ & 6.03072300 & -3.65818800 & -3.14999700 \\
\hline 0 & 0.53434300 & -1.09506900 & -1.30168100 \\
\hline $\mathrm{C}$ & -0.03413400 & 1.08135200 & -2.00540200 \\
\hline $\mathrm{C}$ & -0.46404800 & 0.56199500 & -3.36671400 \\
\hline $\mathrm{H}$ & -1.04206400 & -0.35990800 & -3.25942500 \\
\hline $\mathrm{H}$ & 0.40129900 & 0.35073500 & -4.00559900 \\
\hline $\mathrm{H}$ & -1.09425400 & 1.30051000 & -3.87005800 \\
\hline $\mathrm{H}$ & 0.40082200 & 2.07723700 & -2.04048000 \\
\hline $\mathrm{H}$ & -1.59002300 & 2.41355400 & -1.05504300 \\
\hline $\mathrm{C}$ & -1.51527900 & 1.32682400 & -1.09934900 \\
\hline $\mathrm{C}$ & -2.32161500 & 0.44106200 & -0.65286400 \\
\hline $\mathrm{C}$ & -2.79359500 & -0.86379000 & -0.24576700 \\
\hline $\mathrm{C}$ & -4.13981500 & -1.22299900 & -0.88882900 \\
\hline $\mathrm{C}$ & -6.67198200 & -1.86262200 & -1.93038900 \\
\hline $\mathrm{C}$ & -4.93623400 & -0.25511900 & -1.50186700 \\
\hline $\mathrm{C}$ & -4.63504800 & -2.54182200 & -0.81279000 \\
\hline $\mathrm{C}$ & -5.89718100 & -2.84723300 & -1.32949600 \\
\hline $\mathrm{C}$ & -6.18957000 & -0.55930600 & -2.02708500 \\
\hline $\mathrm{H}$ & -4.55416800 & 0.76013100 & -1.54728700 \\
\hline $\mathrm{H}$ & -6.24570700 & -3.87262000 & -1.25140100 \\
\hline $\mathrm{H}$ & -6.78388300 & 0.21685500 & -2.49866700 \\
\hline $\mathrm{H}$ & -7.65065700 & -2.11769100 & -2.32683300 \\
\hline $\mathrm{C}$ & -2.85719500 & -0.83828800 & 1.29369400 \\
\hline $\mathrm{C}$ & -2.90190900 & -0.60536300 & 4.09343600 \\
\hline $\mathrm{C}$ & -4.06196800 & -0.71829600 & 1.98682300 \\
\hline $\mathrm{C}$ & -1.65905400 & -0.85474500 & 2.03563900 \\
\hline $\mathrm{C}$ & -1.68930400 & -0.73460100 & 3.42563100 \\
\hline
\end{tabular}




$\begin{array}{llll}\mathrm{C} & -4.09510400 & -0.60370400 & 3.37433800 \\ \mathrm{H} & -4.99069700 & -0.70387300 & 1.42371500 \\ \mathrm{H} & -0.74512700 & -0.74706900 & 3.96236000 \\ \mathrm{H} & -5.04726100 & -0.51098700 & 3.88699800 \\ \mathrm{H} & -2.91254000 & -0.51226600 & 5.17545800 \\ 0 & -1.86891900 & -2.00660400 & -0.53543000 \\ \mathrm{H} & -1.32246800 & -1.75367400 & -1.30370200 \\ 0 & -3.94510100 & -3.55164100 & -0.23239900 \\ \mathrm{H} & -3.00283300 & -3.27911200 & -0.21579400 \\ 0 & -0.43449000 & -0.94449500 & 1.44667500 \\ \mathrm{H} & -0.56561200 & -1.31962600 & 0.54835700\end{array}$

Zero-point correction=

0.688895 (Hartree/Particle)

Thermal correction to Energy=

0.729272

Thermal correction to Enthalpy=

0.730217

Thermal correction to Gibbs Free Energy=

0.616666

Sum of electronic and zero-point Energies=

$-2047.194044$

Sum of electronic and thermal Energies=

$-2047.153666$

Sum of electronic and thermal Enthalpies=

$-2047.152722$

Sum of electronic and thermal Free Energies=

$-2047.266272$

$\mathrm{E}(\mathrm{RM} 062 \mathrm{X} / 6-311+\mathrm{G}(2 \mathrm{~d}, \mathrm{p}))=-2048.50458436$

$\begin{array}{crrr}\text { TS2 } & & & \\ \mathrm{N} & 1.91606500 & 1.66454500 & 1.72989400 \\ \mathrm{C} & 2.35399900 & 0.43633300 & 1.69705400 \\ \mathrm{~N} & 2.03988500 & -0.16503500 & 0.51544500 \\ \mathrm{C} & 1.32487700 & 0.73192000 & -0.19989000 \\ \mathrm{~N} & 1.28046200 & 1.83460200 & 0.54838300 \\ \mathrm{C} & 0.71010900 & 3.12795400 & 0.26694600 \\ \mathrm{C} & -0.34284900 & 5.63210300 & -0.22600300 \\ \mathrm{C} & -0.42328200 & 3.51387200 & 0.98159500 \\ \mathrm{C} & 1.37071000 & 3.95264700 & -0.65263100 \\ \mathrm{C} & 0.81201000 & 5.20303200 & -0.89265600 \\ \mathrm{C} & -0.93606700 & 4.78480100 & 0.70662400 \\ \mathrm{H} & 1.29503600 & 5.86589300 & -1.60685000 \\ \mathrm{H} & -1.82874800 & 5.10954500 & 1.23580800 \\ \mathrm{C} & 2.62969200 & 3.50661700 & -1.34939800 \\ \mathrm{H} & 3.08368800 & 4.34604100 & -1.88019700 \\ \mathrm{H} & 2.43013900 & 2.71877600 & -2.08603300 \\ \mathrm{H} & 3.36314400 & 3.11725400 & -0.63480900 \\ \mathrm{C} & -1.07558500 & 2.62404800 & 2.00404400 \\ \mathrm{H} & -2.16383400 & 2.69264600 & 1.92707500 \\ \mathrm{H} & -0.78379200 & 2.93217300 & 3.01477500 \\ \mathrm{H} & -0.79344900 & 1.57834400 & 1.87345400 \\ \mathrm{C} & -0.91261300 & 6.99661800 & -0.50879800\end{array}$




\begin{tabular}{|c|c|c|c|}
\hline $\mathrm{H}$ & -0.21282700 & 7.78135000 & -0.20184200 \\
\hline $\mathrm{H}$ & -1.85345900 & 7.15110000 & 0.02479100 \\
\hline $\mathrm{H}$ & -1.09911500 & 7.12851400 & -1.57940700 \\
\hline $\mathrm{C}$ & 0.67902100 & 0.40407500 & -1.53211400 \\
\hline $\mathrm{C}$ & 2.35221700 & -1.58249800 & 0.24196700 \\
\hline $\mathrm{H}$ & 1.45790400 & -2.03256400 & -0.19795300 \\
\hline $\mathrm{C}$ & 2.72052600 & -2.30198000 & 1.56144900 \\
\hline $\mathrm{H}$ & 1.83377300 & -2.55647400 & 2.15354700 \\
\hline $\mathrm{C}$ & 2.98679600 & -0.33031100 & 2.81579200 \\
\hline $\mathrm{H}$ & 2.19894900 & -0.57523200 & 3.54284300 \\
\hline $\mathrm{H}$ & 3.74564200 & 0.28053600 & 3.30810200 \\
\hline 0 & 3.60629300 & -1.48787500 & 2.31909300 \\
\hline $\mathrm{C}$ & 3.53925900 & -3.50139700 & 1.08098900 \\
\hline $\mathrm{H}$ & 4.21897700 & -3.84492700 & 1.86464800 \\
\hline $\mathrm{H}$ & 2.87141500 & -4.32961000 & 0.81728900 \\
\hline $\mathrm{C}$ & 3.57502600 & -1.82102900 & -0.61972200 \\
\hline $\mathrm{C}$ & 5.86658200 & -2.71166000 & -1.88621800 \\
\hline $\mathrm{C}$ & 4.04550400 & -1.12065300 & -1.72162000 \\
\hline $\mathrm{C}$ & 4.24067700 & -2.95248300 & -0.14066400 \\
\hline $\mathrm{C}$ & 5.38784600 & -3.40992200 & -0.77627800 \\
\hline $\mathrm{C}$ & 5.20580900 & -1.57446400 & -2.35101600 \\
\hline $\mathrm{H}$ & 3.53111500 & -0.23912700 & -2.09077200 \\
\hline $\mathrm{H}$ & 5.91219100 & -4.28671900 & -0.40694300 \\
\hline $\mathrm{H}$ & 5.59576400 & -1.03713800 & -3.20989300 \\
\hline $\mathrm{H}$ & 6.76775900 & -3.05189900 & -2.38740700 \\
\hline 0 & 1.03882600 & -0.64386100 & -2.03823500 \\
\hline $\mathrm{C}$ & -0.36680400 & 1.29677500 & -2.09871100 \\
\hline $\mathrm{C}$ & -0.78040900 & 0.80584600 & -3.48575800 \\
\hline $\mathrm{H}$ & -1.28087600 & -0.16560700 & -3.41426100 \\
\hline $\mathrm{H}$ & 0.07521200 & 0.70522500 & -4.16056900 \\
\hline $\mathrm{H}$ & -1.49063300 & 1.51470000 & -3.91944100 \\
\hline $\mathrm{H}$ & 0.09229300 & 2.28926000 & -2.18566000 \\
\hline $\mathrm{H}$ & -1.91582800 & 2.56277700 & -1.26211300 \\
\hline $\mathrm{C}$ & -1.63426100 & 1.50669500 & -1.19813600 \\
\hline $\mathrm{C}$ & -2.43390600 & 0.65926300 & -0.56397500 \\
\hline $\mathrm{C}$ & -2.48360600 & -0.72974800 & -0.24743200 \\
\hline $\mathrm{C}$ & -3.52137300 & -1.49781200 & -1.06444000 \\
\hline $\mathrm{C}$ & -5.51538000 & -2.90756300 & -2.46527100 \\
\hline $\mathrm{C}$ & -4.44625000 & -0.83482800 & -1.87595400 \\
\hline $\mathrm{C}$ & -3.60418000 & -2.90512700 & -0.97959400 \\
\hline $\mathrm{C}$ & -4.60402300 & -3.59373100 & -1.67250600 \\
\hline $\mathrm{C}$ & -5.43154200 & -1.52122900 & -2.58007100 \\
\hline $\mathrm{H}$ & -4.39128300 & 0.24798200 & -1.92524100 \\
\hline $\mathrm{H}$ & -4.63705400 & -4.67487800 & -1.57794100 \\
\hline
\end{tabular}




$\begin{array}{lccc}\mathrm{H} & -6.13589400 & -0.97451100 & -3.19905900 \\ \mathrm{H} & -6.28697800 & -3.45781300 & -2.99632900 \\ \mathrm{C} & -2.67012300 & -0.91587100 & 1.27134500 \\ \mathrm{C} & -3.12902000 & -0.98614300 & 4.05153200 \\ \mathrm{C} & -3.96402000 & -0.99250200 & 1.79477700 \\ \mathrm{C} & -1.60127100 & -0.87961800 & 2.18279200 \\ \mathrm{C} & -1.83261500 & -0.91089500 & 3.55974600 \\ \mathrm{C} & -4.20337600 & -1.03310300 & 3.16421000 \\ \mathrm{H} & -4.80149700 & -1.00646100 & 1.10271900 \\ \mathrm{H} & -0.97464000 & -0.87804400 & 4.22510000 \\ \mathrm{H} & -5.22200200 & -1.09260800 & 3.53424400 \\ \mathrm{H} & -3.29881500 & -1.01071100 & 5.12392300 \\ 0 & -1.11144900 & -1.62910600 & -0.48827600 \\ \mathrm{H} & -0.88725700 & -1.54088200 & -1.42972100 \\ 0 & -2.74590500 & -3.63837100 & -0.23411700 \\ \mathrm{H} & -1.93861000 & -3.08387400 & -0.12625000 \\ 0 & -0.30433300 & -0.78621700 & 1.76790200 \\ \mathrm{H} & -0.32739100 & -1.04657300 & 0.81135900\end{array}$

Zero-point correction= 0.689743 (Hartree/Particle)

Thermal correction to Energy= 0.729630

0.730575

Thermal correction to Enthalpy=

0.617739

Thermal correction to Gibbs Free Energy=

$-2047.196162$

Sum of electronic and zero-point Energies= $-2047.156275$

Sum of electronic and thermal Energies=

$-2047.155330$

Sum of electronic and thermal Enthalpies=

$-2047.268166$

Sum of electronic and thermal Free Energies=

$\mathrm{E}(\mathrm{RM} 062 \mathrm{X} / 6-311+\mathrm{G}(2 \mathrm{~d}, \mathrm{p}))=-2048.50847708$

TS3B

$\begin{array}{lrrr}\mathrm{C} & 0.94676100 & 0.74455400 & 0.18967300 \\ \mathrm{~N} & 1.99617000 & 1.02170200 & -0.57792200 \\ \mathrm{~N} & -0.08103000 & 1.44591300 & -0.31767600 \\ \mathrm{C} & 0.39192400 & 2.08229300 & -1.42949000 \\ \mathrm{~N} & 1.66652100 & 1.85617800 & -1.59276100 \\ \mathrm{C} & 3.36826100 & 0.65205400 & -0.35251500 \\ \mathrm{C} & 5.99400300 & -0.08021900 & 0.13182400 \\ \mathrm{C} & 4.01375100 & -0.15157900 & -1.29603000 \\ \mathrm{C} & 3.99457200 & 1.14039800 & 0.80349800 \\ \mathrm{C} & 5.31306900 & 0.75209300 & 1.02487600 \\ \mathrm{C} & 5.33469800 & -0.51028800 & -1.01875800 \\ \mathrm{H} & 5.82394000 & 1.11565700 & 1.91351000 \\ \mathrm{H} & 5.85932800 & -1.14330700 & -1.73047900 \\ \mathrm{C} & -1.47917400 & 1.53811600 & 0.13143400 \\ \mathrm{H} & -1.79113200 & 0.53090000 & 0.42621200\end{array}$




\begin{tabular}{|c|c|c|c|}
\hline $\mathrm{C}$ & -2.36040000 & 2.06411900 & -1.02647500 \\
\hline $\mathrm{H}$ & -2.58325600 & 1.29529700 & -1.77360300 \\
\hline $\mathrm{C}$ & -0.54236200 & 2.83197500 & -2.32935500 \\
\hline $\mathrm{H}$ & -0.76688800 & 2.19381900 & -3.19516500 \\
\hline $\mathrm{H}$ & -0.07033600 & 3.75185400 & -2.67947700 \\
\hline 0 & -1.71237400 & 3.18005700 & -1.63044700 \\
\hline $\mathrm{C}$ & -3.58563000 & 2.61513700 & -0.29788500 \\
\hline $\mathrm{H}$ & -4.29494100 & 1.80085000 & -0.10577000 \\
\hline $\mathrm{H}$ & -4.08987600 & 3.37585700 & -0.89894200 \\
\hline $\mathrm{C}$ & -2.98879900 & 3.14694400 & 0.98513100 \\
\hline $\mathrm{C}$ & -1.47301600 & 3.90073000 & 3.18585300 \\
\hline $\mathrm{C}$ & -3.49213000 & 4.10126500 & 1.85920800 \\
\hline $\mathrm{C}$ & -1.74334800 & 2.55799800 & 1.22441500 \\
\hline $\mathrm{C}$ & -0.97115600 & 2.93097300 & 2.31551400 \\
\hline $\mathrm{C}$ & -2.72530200 & 4.47329200 & 2.96502700 \\
\hline $\mathrm{H}$ & -4.45750900 & 4.56481600 & 1.67559400 \\
\hline $\mathrm{H}$ & 0.00144900 & 2.48439100 & 2.49400000 \\
\hline $\mathrm{H}$ & -3.10144600 & 5.22538200 & 3.65212500 \\
\hline $\mathrm{H}$ & -0.88116000 & 4.21027900 & 4.04176100 \\
\hline $\mathrm{C}$ & 3.28894200 & 2.04213300 & 1.78417500 \\
\hline $\mathrm{H}$ & 2.64953100 & 2.77509000 & 1.28120300 \\
\hline $\mathrm{H}$ & 4.02339100 & 2.58756100 & 2.38107000 \\
\hline $\mathrm{H}$ & 2.66694000 & 1.47159900 & 2.48528900 \\
\hline $\mathrm{C}$ & 3.35252900 & -0.59245600 & -2.57267800 \\
\hline $\mathrm{H}$ & 3.51163000 & 0.16061300 & -3.35405400 \\
\hline $\mathrm{H}$ & 2.27387500 & -0.73188600 & -2.46305600 \\
\hline $\mathrm{H}$ & 3.80168200 & -1.52704600 & -2.91965800 \\
\hline $\mathrm{C}$ & 7.40935400 & -0.50540300 & 0.41820000 \\
\hline $\mathrm{H}$ & 8.00099400 & 0.32963700 & 0.80478900 \\
\hline $\mathrm{H}$ & 7.89729400 & -0.89030300 & -0.48070200 \\
\hline $\mathrm{H}$ & 7.42731400 & -1.29702000 & 1.17579000 \\
\hline $\mathrm{C}$ & 0.82834100 & -0.20071100 & 1.37283500 \\
\hline 0 & -0.01683300 & 0.06555500 & 2.19566800 \\
\hline $\mathrm{C}$ & 1.64831100 & -1.46691500 & 1.35176800 \\
\hline $\mathrm{H}$ & 2.70938300 & -1.18411700 & 1.34336000 \\
\hline $\mathrm{C}$ & 1.35749500 & -2.30551000 & 2.59530400 \\
\hline $\mathrm{H}$ & 1.65639900 & -1.78679700 & 3.51201100 \\
\hline $\mathrm{H}$ & 1.90975900 & -3.24699700 & 2.53207500 \\
\hline $\mathrm{H}$ & 0.28965700 & -2.53440600 & 2.65817100 \\
\hline $\mathrm{C}$ & 1.31522700 & -2.17792800 & 0.04467700 \\
\hline $\mathrm{H}$ & 2.11887700 & -2.73217000 & -0.43816500 \\
\hline $\mathrm{C}$ & 0.12752500 & -2.01179600 & -0.55785600 \\
\hline $\mathrm{C}$ & -1.21666800 & -2.10776400 & -0.54199200 \\
\hline $\mathrm{C}$ & -1.96457100 & -1.43772500 & -1.60294600 \\
\hline
\end{tabular}




$\begin{array}{cccc}\mathrm{C} & -2.96941900 & -0.29177300 & -3.94427500 \\ \mathrm{C} & -3.34097400 & -1.44938000 & -1.85424900 \\ \mathrm{C} & -1.06615000 & -0.82386400 & -2.52932600 \\ \mathrm{C} & -1.59858400 & -0.29030400 & -3.72987600 \\ \mathrm{C} & -3.84762800 & -0.85908500 & -3.00931100 \\ \mathrm{H} & -4.02156000 & -1.93715200 & -1.16233700 \\ \mathrm{H} & -0.91310700 & 0.13087200 & -4.46075100 \\ \mathrm{H} & -4.91656800 & -0.86553700 & -3.19791000 \\ \mathrm{H} & -3.36803200 & 0.14877000 & -4.85501200 \\ \mathrm{C} & -1.88531400 & -2.93182200 & 0.50604700 \\ \mathrm{C} & -3.25510600 & -4.42202200 & 2.46743900 \\ \mathrm{C} & -1.41947100 & -4.20389200 & 0.90153200 \\ \mathrm{C} & -3.01304000 & -2.41855000 & 1.16357800 \\ \mathrm{C} & -3.69985000 & -3.14505900 & 2.12973200 \\ \mathrm{C} & -2.11678000 & -4.93977600 & 1.86340000 \\ \mathrm{H} & -3.35223900 & -1.41828800 & 0.90700100 \\ \mathrm{H} & -4.57110800 & -2.71587300 & 2.61412900 \\ \mathrm{H} & -1.73261200 & -5.91975800 & 2.12938900 \\ \mathrm{H} & -3.78199900 & -5.00834800 & 3.21440400 \\ 0 & 0.18154200 & -0.82950000 & -2.20611200 \\ \mathrm{O} & -0.31088800 & -4.78023200 & 0.37607500 \\ \mathrm{H} & 0.28977200 & -4.06934700 & 0.07749700\end{array}$

Zero-point correction $=$

Thermal correction to Energy=

0.662900 (Hartree/Particle)
0.701225
0.702170
0.593567
-1970.866684
-1970.828358
-1970.827414
-1970.936017

Thermal correction to Enthalpy=

Thermal correction to Gibbs Free Energy=

Sum of electronic and zero-point Energies=

Sum of electronic and thermal Energies=

Sum of electronic and thermal Enthalpies=

Sum of electronic and thermal Free Energies=

$-1970.936017$

$\mathrm{E}(\mathrm{RM} 062 \mathrm{X} / 6-311+\mathrm{G}(2 \mathrm{~d}, \mathrm{p}))=-1972.11289944$

TS3

$\begin{array}{lrrr}\mathrm{C} & -1.29034000 & 0.54646500 & 0.01185000 \\ \mathrm{~N} & -2.56037100 & 0.80337600 & 0.30789000 \\ \mathrm{~N} & -0.59786500 & 1.64009500 & 0.39593700 \\ \mathrm{C} & -1.49472200 & 2.49029600 & 0.98060200 \\ \mathrm{~N} & -2.70320600 & 2.01207200 & 0.92781200 \\ \mathrm{C} & -3.74100500 & 0.03762700 & 0.00568600 \\ \mathrm{C} & -6.01394800 & -1.41427600 & -0.58140000 \\ \mathrm{C} & -4.31669900 & 0.19791900 & -1.25496400 \\ \mathrm{C} & -4.27379600 & -0.78263600 & 1.00574200 \\ \mathrm{C} & -5.41567300 & -1.50967500 & 0.67973800 \\ \mathrm{C} & -5.46041300 & -0.55380100 & -1.52999200\end{array}$




\begin{tabular}{|c|c|c|c|}
\hline $\mathrm{H}$ & -5.85070000 & -2.16789700 & 1.42858900 \\
\hline $\mathrm{H}$ & -5.92970800 & -0.45694800 & -2.50581000 \\
\hline $\mathrm{C}$ & 0.84632200 & 1.92095300 & 0.34172200 \\
\hline $\mathrm{H}$ & 1.37000000 & 0.99691400 & 0.60220600 \\
\hline $\mathrm{C}$ & 1.19782000 & 3.02378500 & 1.36555600 \\
\hline $\mathrm{H}$ & 1.23259800 & 2.64087400 & 2.39259200 \\
\hline $\mathrm{C}$ & -1.03038400 & 3.72723600 & 1.69046300 \\
\hline $\mathrm{H}$ & -1.04887900 & 3.52652800 & 2.77182400 \\
\hline $\mathrm{H}$ & -1.70391600 & 4.55943100 & 1.47806500 \\
\hline 0 & 0.25741300 & 4.08692200 & 1.26016200 \\
\hline $\mathrm{C}$ & 2.52910000 & 3.56138000 & 0.84229800 \\
\hline $\mathrm{H}$ & 3.34795000 & 2.92670700 & 1.20205900 \\
\hline $\mathrm{H}$ & 2.70376900 & 4.58303700 & 1.18835000 \\
\hline $\mathrm{C}$ & 2.35974500 & 3.44009000 & -0.65517100 \\
\hline $\mathrm{C}$ & 1.65051600 & 2.94405200 & -3.29265000 \\
\hline $\mathrm{C}$ & 3.02443300 & 4.11191000 & -1.67229100 \\
\hline $\mathrm{C}$ & 1.35845700 & 2.51250900 & -0.95816000 \\
\hline $\mathrm{C}$ & 0.99060900 & 2.25726600 & -2.27155900 \\
\hline $\mathrm{C}$ & 2.66242400 & 3.85672400 & -2.99633300 \\
\hline $\mathrm{H}$ & 3.80162300 & 4.83513500 & -1.44142700 \\
\hline $\mathrm{H}$ & 0.21128000 & 1.53954300 & -2.50430000 \\
\hline $\mathrm{H}$ & 3.16668400 & 4.38126800 & -3.80246600 \\
\hline $\mathrm{H}$ & 1.37286600 & 2.76338200 & -4.32659100 \\
\hline $\mathrm{C}$ & -3.73924600 & 1.15908900 & -2.25991300 \\
\hline $\mathrm{H}$ & -4.32041700 & 1.14084600 & -3.18437800 \\
\hline $\mathrm{H}$ & -2.70028900 & 0.91488800 & -2.50954900 \\
\hline $\mathrm{H}$ & -3.74723300 & 2.18247400 & -1.86804000 \\
\hline $\mathrm{C}$ & -3.62722700 & -0.86705200 & 2.36104500 \\
\hline $\mathrm{H}$ & -2.55004100 & -1.05826900 & 2.27962100 \\
\hline $\mathrm{H}$ & -4.08854100 & -1.66108700 & 2.95246100 \\
\hline $\mathrm{H}$ & -3.75128600 & 0.07748600 & 2.90405300 \\
\hline $\mathrm{C}$ & -7.23871700 & -2.23018400 & -0.89839800 \\
\hline $\mathrm{H}$ & -7.64609800 & -1.96959500 & -1.87814500 \\
\hline $\mathrm{H}$ & -8.01946000 & -2.07288900 & -0.14750200 \\
\hline $\mathrm{H}$ & -7.00230800 & -3.29972900 & -0.90190600 \\
\hline $\mathrm{C}$ & -0.62492700 & -0.68619500 & -0.57889500 \\
\hline 0 & 0.43528400 & -0.46718900 & -1.15090000 \\
\hline $\mathrm{C}$ & -1.37960400 & -1.99734000 & -0.76429000 \\
\hline $\mathrm{H}$ & -2.27210800 & -1.98935200 & -0.13277200 \\
\hline $\mathrm{C}$ & -1.79466000 & -2.14093600 & -2.23516800 \\
\hline $\mathrm{H}$ & -2.31015100 & -3.09503300 & -2.37860500 \\
\hline $\mathrm{H}$ & -0.90848500 & -2.12479400 & -2.87600400 \\
\hline $\mathrm{H}$ & -2.47329500 & -1.34226700 & -2.55018900 \\
\hline $\mathrm{C}$ & -0.54562900 & -3.18324300 & -0.29682700 \\
\hline
\end{tabular}




$\begin{array}{lrrr}\mathrm{H} & -1.08179000 & -4.12563800 & -0.19580900 \\ \mathrm{C} & 0.69340400 & -3.03207400 & 0.08152200 \\ \mathrm{C} & 1.84284600 & -2.51545400 & 0.46985700 \\ \mathrm{C} & 3.07909600 & -2.72709000 & -0.34341100 \\ \mathrm{C} & 5.49211500 & -3.10449400 & -1.71862400 \\ \mathrm{C} & 3.72888200 & -1.63872700 & -0.95496900 \\ \mathrm{C} & 3.64438200 & -3.99873400 & -0.46666900 \\ \mathrm{C} & 4.84132900 & -4.19791200 & -1.15081800 \\ \mathrm{C} & 4.93687200 & -1.83202800 & -1.62636400 \\ \mathrm{H} & 3.13637000 & -4.83538800 & 0.00526200 \\ \mathrm{H} & 5.26560400 & -5.19389900 & -1.22815300 \\ \mathrm{H} & 5.41458200 & -0.97255700 & -2.08659900 \\ \mathrm{H} & 6.43095700 & -3.24100800 & -2.24756200 \\ \mathrm{O} & 3.22250300 & -0.38062600 & -0.91735400 \\ \mathrm{H} & 2.25247000 & -0.42912100 & -0.81807300 \\ \mathrm{C} & 1.91612300 & -1.65878600 & 1.68379000 \\ \mathrm{C} & 2.14112700 & 0.03520500 & 3.91318200 \\ \mathrm{C} & 0.75662900 & -0.93397700 & 2.12550100 \\ \mathrm{C} & 3.13376300 & -1.49397800 & 2.35290800 \\ \mathrm{C} & 3.26545300 & -0.65785200 & 3.45712700 \\ \mathrm{C} & 0.92265100 & -0.09837300 & 3.26311900 \\ \mathrm{H} & 4.00051700 & -2.04224700 & 1.99027900 \\ \mathrm{H} & 4.22376700 & -0.55310300 & 3.95573300 \\ \mathrm{H} & 0.04271200 & 0.43528000 & 3.61575400 \\ \mathrm{H} & 2.21785800 & 0.68707100 & 4.78046000 \\ \mathrm{O} & -0.40513300 & -1.00847800 & 1.54134900\end{array}$

Zero-point correction=

0.663367 (Hartree/Particle)
0.701711
0.702655
0.593567
-1970.874796
-1970.836452
-1970.835508
-1970.944596

Thermal correction to Energy=

Thermal correction to Enthalpy=

Thermal correction to Gibbs Free Energy=

Sum of electronic and zero-point Energies=

Sum of electronic and thermal Energies=

Sum of electronic and thermal Enthalpies=

Sum of electronic and thermal Free Energies=

$\mathrm{E}(\mathrm{RM} 062 \mathrm{X} / 6-311+\mathrm{G}(2 \mathrm{~d}, \mathrm{p}))=-1972.12023455$

TS4B

$\begin{array}{lrrr}\mathrm{C} & -1.24153100 & 0.65091500 & 0.00189300 \\ \mathrm{~N} & -2.54399700 & 0.82750200 & 0.21032600 \\ \mathrm{~N} & -0.67147700 & 1.84503500 & 0.26939900 \\ \mathrm{C} & -1.66650900 & 2.67696800 & 0.70123800 \\ \mathrm{~N} & -2.82504100 & 2.08886300 & 0.66049200 \\ \mathrm{C} & -3.64291100 & -0.07352700 & -0.02121400 \\ \mathrm{C} & -5.75777800 & -1.79305300 & -0.46820300\end{array}$




\begin{tabular}{|c|c|c|c|}
\hline $\mathrm{C}$ & -4.20224100 & -0.11104200 & -1.29844400 \\
\hline $\mathrm{C}$ & -4.11779600 & -0.82868700 & 1.05632700 \\
\hline $\mathrm{C}$ & -5.17972000 & -1.69198500 & 0.80215100 \\
\hline $\mathrm{C}$ & -5.26414800 & -0.99533600 & -1.50057200 \\
\hline $\mathrm{H}$ & -5.56661500 & -2.30203600 & 1.61534200 \\
\hline $\mathrm{H}$ & -5.71768800 & -1.05374300 & -2.48690200 \\
\hline $\mathrm{C}$ & -3.69981300 & 0.78595400 & -2.39812000 \\
\hline $\mathrm{H}$ & -2.64217900 & 0.60680000 & -2.61927200 \\
\hline $\mathrm{H}$ & -3.79927800 & 1.84026000 & -2.11652300 \\
\hline $\mathrm{H}$ & -4.26868100 & 0.62145100 & -3.31558400 \\
\hline $\mathrm{C}$ & -3.48267500 & -0.71311800 & 2.41460700 \\
\hline $\mathrm{H}$ & -2.40067500 & -0.88567600 & 2.36091400 \\
\hline $\mathrm{H}$ & -3.92535100 & -1.43353000 & 3.10620600 \\
\hline $\mathrm{H}$ & -3.63067500 & 0.29231800 & 2.82602900 \\
\hline $\mathrm{C}$ & -6.90433400 & -2.74100000 & -0.70059000 \\
\hline $\mathrm{H}$ & -6.61631100 & -3.76911000 & -0.45740800 \\
\hline $\mathrm{H}$ & -7.23642400 & -2.71585000 & -1.74132500 \\
\hline $\mathrm{H}$ & -7.75709700 & -2.48395400 & -0.06341600 \\
\hline $\mathrm{C}$ & 0.74401200 & 2.24738700 & 0.22561700 \\
\hline $\mathrm{H}$ & 1.33257200 & 1.40560300 & 0.59832600 \\
\hline $\mathrm{C}$ & 0.95066700 & 3.49893000 & 1.10848600 \\
\hline $\mathrm{H}$ & 0.97851000 & 3.25662000 & 2.17847000 \\
\hline $\mathrm{C}$ & -1.34737300 & 4.03169600 & 1.25956300 \\
\hline $\mathrm{H}$ & -1.40452700 & 3.97563200 & 2.35641000 \\
\hline $\mathrm{H}$ & -2.07706600 & 4.76482700 & 0.91092600 \\
\hline 0 & -0.07491400 & 4.44923100 & 0.83780600 \\
\hline $\mathrm{C}$ & 2.24201200 & 4.10277400 & 0.55619200 \\
\hline $\mathrm{H}$ & 3.10900600 & 3.61258000 & 1.01414900 \\
\hline $\mathrm{H}$ & 2.29619900 & 5.17286500 & 0.77187800 \\
\hline $\mathrm{C}$ & 2.14193600 & 3.77810800 & -0.91751400 \\
\hline $\mathrm{C}$ & 1.59912300 & 2.87247500 & -3.48455600 \\
\hline $\mathrm{C}$ & 2.77566900 & 4.38186900 & -1.99597400 \\
\hline $\mathrm{C}$ & 1.25230300 & 2.71991300 & -1.12334100 \\
\hline $\mathrm{C}$ & 0.97229800 & 2.25514100 & -2.40030100 \\
\hline $\mathrm{C}$ & 2.49609200 & 3.92185200 & -3.28415500 \\
\hline $\mathrm{H}$ & 3.46582200 & 5.20681200 & -1.84164000 \\
\hline $\mathrm{H}$ & 0.29474900 & 1.42149700 & -2.54951000 \\
\hline $\mathrm{H}$ & 2.97597800 & 4.39006500 & -4.13854600 \\
\hline $\mathrm{H}$ & 1.38852100 & 2.52894100 & -4.49283600 \\
\hline $\mathrm{C}$ & -0.39923800 & -0.59105400 & -0.33647900 \\
\hline 0 & 0.65274600 & -0.33368600 & -0.91982800 \\
\hline $\mathrm{C}$ & -1.07800600 & -1.95674300 & -0.48885900 \\
\hline $\mathrm{C}$ & -0.28658600 & -3.06241200 & 0.25517000 \\
\hline $\mathrm{H}$ & -0.52046000 & -3.01205700 & 1.31726500 \\
\hline
\end{tabular}




$\begin{array}{lrrr}\mathrm{C} & 1.18814300 & -2.96126400 & 0.08034700 \\ \mathrm{C} & 2.16396300 & -2.26858400 & 0.73349700 \\ \mathrm{O} & 1.67554900 & -3.62512900 & -1.01825700 \\ \mathrm{C} & 3.37561400 & -2.49795100 & -0.04333800 \\ \mathrm{C} & 5.18165700 & -3.36245300 & -2.00245000 \\ \mathrm{C} & 4.70465100 & -2.04780800 & -0.00556700 \\ \mathrm{C} & 2.99960700 & -3.34252000 & -1.09648500 \\ \mathrm{C} & 3.86585200 & -3.80379500 & -2.07834600 \\ \mathrm{C} & 5.58951600 & -2.48701900 & -0.98284500 \\ \mathrm{H} & 5.04082900 & -1.35427900 & 0.75769700 \\ \mathrm{H} & 3.51983200 & -4.46357300 & -2.86711400 \\ \mathrm{H} & 6.61872700 & -2.14161300 & -0.96114400 \\ \mathrm{H} & 5.89945800 & -3.68970900 & -2.74822000 \\ \mathrm{C} & 2.06488600 & -1.37819800 & 1.90851400 \\ \mathrm{C} & 2.09302000 & 0.38614900 & 4.11712300 \\ \mathrm{C} & 0.89976100 & -0.61286200 & 2.21825700 \\ \mathrm{C} & 3.17667500 & -1.26755000 & 2.75734000 \\ \mathrm{C} & 3.21052800 & -0.40354400 & 3.84763700 \\ \mathrm{C} & 0.96601100 & 0.27941500 & 3.31452700 \\ \mathrm{H} & 4.03678600 & -1.90247000 & 2.56534300 \\ \mathrm{H} & 4.09287800 & -0.35497800 & 4.47790400 \\ \mathrm{H} & 0.07242100 & 0.86357600 & 3.52360700 \\ \mathrm{H} & 2.09539700 & 1.07475900 & 4.95832000 \\ \mathrm{O} & -0.24534200 & -0.72250600 & 1.57771900 \\ \mathrm{H} & -2.06997500 & -1.93272700 & -0.03386300 \\ \mathrm{C} & -1.21594600 & -2.25909800 & -1.98171500 \\ \mathrm{H} & -1.73594900 & -3.21033200 & -2.13051800 \\ \mathrm{H} & -0.22879900 & -2.32095200 & -2.44849500 \\ \mathrm{H} & -1.78769700 & -1.47956100 & -2.49777200 \\ -0.61728600 & -4.03521300 & -0.12624200\end{array}$

Zero-point correction $=$

Thermal correction to Energy=

0.667007 (Hartree/Particle)

0.704030

0.704974

Thermal correction to Enthalpy=

0.599039

Thermal correction to Gibbs Free Energy=

$-1970.933593$

Sum of electronic and zero-point Energies=

$-1970.896571$

$-1970.895626$

Sum of electronic and thermal Enthalpies=

$-1971.001561$

Sum of electronic and thermal Free Energies=

$\mathrm{E}(\mathrm{RM} 062 \mathrm{X} / 6-311+\mathrm{G}(2 \mathrm{~d}, \mathrm{p}))=-1972.17084056$

TS4

$\begin{array}{llll}\mathrm{N} & -3.47244400 & 1.39078600 & 0.96091500 \\ \mathrm{C} & -2.49294300 & 2.23638500 & 1.06552300 \\ \mathrm{~N} & -1.33544600 & 1.70933000 & 0.55524800\end{array}$




\begin{tabular}{|c|c|c|c|}
\hline $\mathrm{C}$ & -1.57583200 & 0.44982800 & 0.10293800 \\
\hline $\mathrm{N}$ & -2.87928700 & 0.29996800 & 0.36659000 \\
\hline $\mathrm{C}$ & -0.07967200 & 2.45858700 & 0.48361400 \\
\hline $\mathrm{H}$ & 0.72010300 & 1.80930000 & 0.85462600 \\
\hline $\mathrm{C}$ & 0.10203000 & -0.79966300 & -0.07099200 \\
\hline 0 & 0.96192000 & -0.15383000 & -0.66612700 \\
\hline $\mathrm{C}$ & -0.54012700 & -2.05924600 & -0.65866700 \\
\hline $\mathrm{H}$ & -1.48908900 & -2.23720000 & -0.14419800 \\
\hline $\mathrm{C}$ & 0.36852100 & -3.23170500 & -0.27557300 \\
\hline $\mathrm{H}$ & -0.08272800 & -4.21569800 & -0.17093500 \\
\hline $\mathrm{C}$ & 1.60767400 & -2.96759100 & 0.04363500 \\
\hline $\mathrm{C}$ & 2.65834200 & -2.26055400 & 0.40936500 \\
\hline $\mathrm{C}$ & 3.88857300 & -2.19553100 & -0.42725100 \\
\hline $\mathrm{C}$ & 6.25582000 & -2.05693900 & -1.91227500 \\
\hline $\mathrm{C}$ & 4.61310200 & -3.35191700 & -0.72975500 \\
\hline $\mathrm{C}$ & 4.34770300 & -0.95428900 & -0.91223400 \\
\hline $\mathrm{C}$ & 5.54019200 & -0.89664500 & -1.63849300 \\
\hline $\mathrm{C}$ & 5.79025400 & -3.29454000 & -1.46992400 \\
\hline $\mathrm{H}$ & 4.24566800 & -4.30277600 & -0.35318000 \\
\hline $\mathrm{H}$ & 5.87627900 & 0.07104200 & -1.99811400 \\
\hline $\mathrm{H}$ & 6.34236300 & -4.20284400 & -1.68863700 \\
\hline $\mathrm{H}$ & 7.17794600 & -1.99279600 & -2.48270800 \\
\hline $\mathrm{C}$ & 2.57437500 & -1.40342000 & 1.62844800 \\
\hline $\mathrm{C}$ & 2.49006500 & 0.30929100 & 3.86116300 \\
\hline $\mathrm{C}$ & 1.38175700 & -0.74120500 & 1.98704900 \\
\hline $\mathrm{C}$ & 3.71615000 & -1.15719200 & 2.39944700 \\
\hline $\mathrm{C}$ & 3.68278500 & -0.31868300 & 3.50791300 \\
\hline $\mathrm{C}$ & 1.34645500 & 0.10071500 & 3.09720300 \\
\hline $\mathrm{H}$ & 4.64521600 & -1.64330800 & 2.11359200 \\
\hline $\mathrm{H}$ & 4.58358100 & -0.15528700 & 4.09078100 \\
\hline $\mathrm{H}$ & 0.40442900 & 0.58238800 & 3.34434900 \\
\hline $\mathrm{H}$ & 2.44939600 & 0.96713600 & 4.72391500 \\
\hline 0 & 3.68842200 & 0.20427100 & -0.69822200 \\
\hline $\mathrm{H}$ & 2.73810600 & 0.04104400 & -0.50861700 \\
\hline 0 & 0.18267200 & -0.93862900 & 1.34042200 \\
\hline $\mathrm{C}$ & -0.78439400 & -1.90436100 & -2.15126600 \\
\hline $\mathrm{H}$ & 0.16026900 & -1.73372700 & -2.67497600 \\
\hline $\mathrm{H}$ & -1.44601800 & -1.05022500 & -2.33787900 \\
\hline $\mathrm{H}$ & -1.26024100 & -2.80050100 & -2.55981400 \\
\hline $\mathrm{C}$ & -3.68677300 & -0.83979800 & 0.03949000 \\
\hline $\mathrm{C}$ & -5.21459800 & -3.05881900 & -0.61313400 \\
\hline $\mathrm{C}$ & -3.81752400 & -1.86233000 & 0.98545900 \\
\hline $\mathrm{C}$ & -4.31319200 & -0.87360700 & -1.20751400 \\
\hline $\mathrm{C}$ & -5.07226500 & -2.00470400 & -1.51579400 \\
\hline
\end{tabular}




\begin{tabular}{cccc}
$\mathrm{C}$ & -4.58670700 & -2.96897200 & 0.63344100 \\
$\mathrm{H}$ & -5.56720200 & -2.05744600 & -2.48264300 \\
$\mathrm{H}$ & -4.69920900 & -3.78098300 & 1.34867500 \\
$\mathrm{C}$ & -4.19356300 & 0.28176000 & -2.16500000 \\
$\mathrm{H}$ & -4.68044200 & 0.04706800 & -3.11430400 \\
$\mathrm{H}$ & -3.14710800 & 0.53455100 & -2.36667800 \\
$\mathrm{H}$ & -4.66600200 & 1.17903700 & -1.74953400 \\
$\mathrm{C}$ & -3.12456700 & -1.76414100 & 2.31765500 \\
$\mathrm{H}$ & -2.03552600 & -1.72804100 & 2.19274300 \\
$\mathrm{H}$ & -3.37263100 & -2.62313200 & 2.94529000 \\
$\mathrm{H}$ & -3.42028400 & -0.85174000 & 2.84707400 \\
$\mathrm{C}$ & -6.01843700 & -4.28193500 & -0.96970300 \\
$\mathrm{H}$ & -5.36204800 & -5.14476100 & -1.12851700 \\
$\mathrm{H}$ & -6.59471900 & -4.12562200 & -1.88515300 \\
$\mathrm{H}$ & -6.71299400 & -4.54542700 & -0.16598300 \\
$\mathrm{C}$ & -0.16899300 & 3.74894100 & 1.33605700 \\
$\mathrm{H}$ & 0.01418700 & 3.55676300 & 2.40135200 \\
$\mathrm{C}$ & -2.49447600 & 3.59386400 & 1.69693400 \\
$\mathrm{H}$ & -2.39464600 & 3.48901400 & 2.78744300 \\
$\mathrm{H}$ & -3.43087700 & 4.11173100 & 1.48174600 \\
$\mathrm{O}$ & -1.44050700 & 4.36261900 & 1.16531100 \\
$\mathrm{C}$ & 0.85284100 & 4.68418400 & 0.68421700 \\
$\mathrm{H}$ & 1.84744900 & 4.50896700 & 1.11059600 \\
$\mathrm{H}$ & 0.58608800 & 5.72986300 & 0.85813800 \\
$\mathrm{C}$ & 0.80529300 & 4.27052500 & -0.76938000 \\
$\mathrm{C}$ & 0.51620500 & 3.08679300 & -3.26619700 \\
$\mathrm{C}$ & 1.21348600 & 4.96764400 & -1.90005200 \\
$\mathrm{C}$ & 0.25864200 & 2.99179400 & -0.89226300 \\
$\mathrm{H}$ & 0.11317600 & 2.38223900 & -2.13049400 \\
$\mathrm{H}$ & 1.06105900 & 4.36673100 & -3.15101500 \\
\hline & -0.29056100 & 1.37625800 & -2.20749300 \\
$\mathrm{H}$ & 1.36730300 & 4.90229100 & -4.04486600 \\
$\mathrm{H}$ & -40756000 & 2.63409500 & -4.24714800
\end{tabular}

Zero-point correction=

0.663105 (Hartree/Particle)

Thermal correction to Energy=

0.701308

Thermal correction to Enthalpy=

0.702253

Thermal correction to Gibbs Free Energy=

0.592065

Sum of electronic and zero-point Energies=

$-1970.872513$

$-1970.834309$

$-1970.833365$

Sum of electronic and thermal Enthalpies=

$-1970.943553$

Sum of electronic and thermal Free Energies=

$\mathrm{E}(\mathrm{RM} 062 \mathrm{X} / 6-311+\mathrm{G}(2 \mathrm{~d}, \mathrm{p}))=-1972.11496751$ 
TS5B

\begin{tabular}{|c|c|c|c|}
\hline $\mathrm{C}$ & 1.49964700 & 0.39211000 & -0.41220700 \\
\hline $\mathrm{N}$ & 2.73436200 & 0.14408100 & -0.86945100 \\
\hline $\mathrm{N}$ & 1.11702400 & 1.43882600 & -1.19268700 \\
\hline $\mathrm{C}$ & 2.12529500 & 1.75066300 & -2.06770900 \\
\hline $\mathrm{N}$ & 3.14555500 & 0.96719600 & -1.89318400 \\
\hline $\mathrm{C}$ & 3.63722300 & -0.85555600 & -0.37776600 \\
\hline $\mathrm{C}$ & 5.35870200 & -2.80833900 & 0.57746400 \\
\hline $\mathrm{C}$ & 3.82496600 & -2.01495400 & -1.13995400 \\
\hline $\mathrm{C}$ & 4.29474500 & -0.62822100 & 0.83179600 \\
\hline $\mathrm{C}$ & 5.15294300 & -1.62981600 & 1.29338000 \\
\hline $\mathrm{C}$ & 4.69098500 & -2.98325700 & -0.63929300 \\
\hline $\mathrm{H}$ & 5.67383000 & -1.47929500 & 2.23605600 \\
\hline $\mathrm{H}$ & 4.84690600 & -3.89731100 & -1.20864900 \\
\hline $\mathrm{C}$ & -0.12346100 & 2.20712400 & -1.10252000 \\
\hline $\mathrm{H}$ & -0.95263400 & 1.49511800 & -1.06484000 \\
\hline $\mathrm{C}$ & -0.27256000 & 3.15367000 & -2.31795800 \\
\hline $\mathrm{H}$ & -0.66430300 & 2.63704400 & -3.20351900 \\
\hline $\mathrm{C}$ & 1.92776100 & 2.82513700 & -3.09278800 \\
\hline $\mathrm{H}$ & 1.59572300 & 2.36950600 & -4.03737100 \\
\hline $\mathrm{H}$ & 2.86231300 & 3.35887500 & -3.27351600 \\
\hline 0 & 0.97909900 & 3.75596900 & -2.62364300 \\
\hline $\mathrm{C}$ & -1.18061900 & 4.27020100 & -1.79380500 \\
\hline $\mathrm{H}$ & -2.23230400 & 3.98744700 & -1.92057800 \\
\hline $\mathrm{H}$ & -1.00900000 & 5.20017100 & -2.34225100 \\
\hline $\mathrm{C}$ & -0.80851000 & 4.34432000 & -0.32975600 \\
\hline $\mathrm{C}$ & 0.10034100 & 4.03734500 & 2.27726900 \\
\hline $\mathrm{C}$ & -1.00070100 & 5.37719900 & 0.58012900 \\
\hline $\mathrm{C}$ & -0.17833900 & 3.16434000 & 0.07017800 \\
\hline $\mathrm{C}$ & 0.28689500 & 2.99446500 & 1.36727100 \\
\hline $\mathrm{C}$ & -0.54167200 & 5.21426900 & 1.88862500 \\
\hline $\mathrm{H}$ & -1.48649600 & 6.30065200 & 0.27671000 \\
\hline $\mathrm{H}$ & 0.77723800 & 2.07123000 & 1.66967100 \\
\hline $\mathrm{H}$ & -0.67677300 & 6.01566200 & 2.60908200 \\
\hline $\mathrm{H}$ & 0.45932000 & 3.93134800 & 3.29693800 \\
\hline $\mathrm{C}$ & 4.08342500 & 0.64499700 & 1.60478600 \\
\hline $\mathrm{H}$ & 4.16821100 & 1.52196300 & 0.95388500 \\
\hline $\mathrm{H}$ & 4.82555300 & 0.73434900 & 2.40196200 \\
\hline $\mathrm{H}$ & 3.08517800 & 0.65212200 & 2.05569500 \\
\hline $\mathrm{C}$ & 3.10520900 & -2.19847800 & -2.44869700 \\
\hline $\mathrm{H}$ & 3.48417600 & -1.50374400 & -3.20615300 \\
\hline $\mathrm{H}$ & 2.03169800 & -2.00391100 & -2.34285400 \\
\hline $\mathrm{H}$ & 3.23641400 & -3.21704100 & -2.82115900 \\
\hline $\mathrm{C}$ & 6.28252000 & -3.88044400 & 1.09442200 \\
\hline
\end{tabular}




$\begin{array}{cccc}\mathrm{H} & 6.71158100 & -3.60198100 & 2.06021400 \\ \mathrm{H} & 7.10589700 & -4.06055700 & 0.39505300 \\ \mathrm{H} & 5.74979600 & -4.82916000 & 1.21862200 \\ \mathrm{C} & 0.47979100 & -0.61358200 & 1.18524000 \\ \mathrm{O} & 1.08211400 & -0.40222700 & 2.22137600 \\ \mathrm{C} & 0.41755600 & -2.00373600 & 0.54824100 \\ \mathrm{H} & 1.44971600 & -2.24928600 & 0.27351400 \\ \mathrm{C} & -0.40453300 & -2.06854800 & -0.73776700 \\ \mathrm{C} & -1.90331000 & -2.00746900 & -0.67460400 \\ \mathrm{C} & -2.88109700 & -1.53587900 & 0.15955400 \\ \mathrm{C} & -4.14032200 & -1.78111100 & -0.53355000 \\ \mathrm{C} & -6.04755200 & -2.52589800 & -2.43438500 \\ \mathrm{C} & -5.49320600 & -1.49760300 & -0.29452200 \\ \mathrm{C} & -3.79571400 & -2.40255800 & -1.73721500 \\ \mathrm{C} & -4.71113300 & -2.79544800 & -2.70444300 \\ \mathrm{C} & -6.42949000 & -1.87733900 & -1.24815900 \\ \mathrm{H} & -5.80825400 & -0.97847700 & 0.60458500 \\ \mathrm{H} & -4.38990000 & -3.27929700 & -3.62063400 \\ \mathrm{H} & -7.47987300 & -1.66394900 & -1.07543500 \\ \mathrm{H} & -6.80606000 & -2.81233400 & -3.15606700 \\ \mathrm{H} & -1.05163300 & -2.86451400 & 1.93556200 \\ \mathrm{C} & -2.78047600 & -0.90905700 & 1.48927500 \\ \mathrm{C} & -2.64805000 & 0.31082200 & 4.02326400 \\ \mathrm{C} & -1.70790800 & -0.07474200 & 1.83846300 \\ \mathrm{C} & -3.77281400 & -1.13318000 & 2.45462200 \\ \mathrm{C} & -3.71161400 & -0.53187400 & 3.70768200 \\ \mathrm{C} & -1.64201600 & 0.53542400 & 3.08843300 \\ \mathrm{H} & -4.58818800 & -1.81238300 & 2.22653600 \\ \mathrm{H} & -4.49169300 & -0.72690600 & 4.43692700 \\ \mathrm{H} & -0.79337700 & 1.17164200 & 3.31077300 \\ \mathrm{H} & -2.59436400 & 0.78571900 & 4.99813400 \\ \mathrm{H} & -0.71793900 & -4.04017000 & 1.17284000\end{array}$

Zero-point correction $=$

0.666801 (Hartree/Particle)

Thermal correction to Energy=

0.703837

0.704782

Thermal correction to Enthalpy=

0.598258

$-1970.930474$

$-1970.893437$ 
Sum of electronic and thermal Enthalpies=

$-1970.892493$

Sum of electronic and thermal Free Energies=

$-1970.999016$

$\mathrm{E}(\mathrm{RM} 062 \mathrm{X} / 6-311+\mathrm{G}(2 \mathrm{~d}, \mathrm{p}))=-1972.16646892$

TS5

$\begin{array}{llll}\text { C } & 0.60822400 & -0.98649300 & -0.06624200\end{array}$

$\mathrm{H} \quad 0.58055700 \quad-0.46271500 \quad-1.02322200$

$\mathrm{N} \quad 1.68187300 \quad-1.32766700 \quad 0.63383500$

$\mathrm{N} \quad-0.44956100 \quad-1.39816200 \quad 0.64031600$

C $\quad 0.03655000 \quad-1.97543500 \quad 1.78224400$

$\begin{array}{llll}\mathrm{N} & 1.33825000 & -1.95046000 & 1.79815900\end{array}$

$\begin{array}{llll}\text { C } & 3.06161300 & -1.16464500 & 0.26201100\end{array}$

$\begin{array}{llll}\text { C } & 5.73246800 & -0.86885100 & -0.44161500\end{array}$

$\begin{array}{llll}\text { C } & 3.46877400 & -1.57914500 & -1.01211800\end{array}$

$\begin{array}{llll}\text { C } & 3.94102000 & -0.59430600 & 1.19275800\end{array}$

$\begin{array}{llll}\text { C } & 5.27442200 & -0.46301200 & 0.81292600\end{array}$

$\begin{array}{llll}\text { C } & 4.81715800 & -1.41984500 & -1.33566400\end{array}$

$\mathrm{H} \quad 5.97173000 \quad-0.01427300 \quad 1.51696000$

$\begin{array}{llll}\mathrm{H} & 5.15451200 & -1.73616700 & -2.31977500\end{array}$

$\begin{array}{llll}\text { C } & -1.87465500 & -1.29541100 & 0.30345600\end{array}$

$\begin{array}{llll}\mathrm{H} & -2.07545200 & -0.25590500 & 0.02777200\end{array}$

$\begin{array}{llll}\text { C } & -2.73410000 & -1.71156900 & 1.52199400\end{array}$

$\begin{array}{llll}\mathrm{H} & -2.87272500 & -0.88006500 & 2.22476700\end{array}$

$\begin{array}{llll}\text { C } & -0.92274600 & -2.47449100 & 2.81790500\end{array}$

$\mathrm{H} \quad-1.07308300 \quad-1.68875100 \quad 3.57311300$

$\begin{array}{llll}\mathrm{H} & -0.52698700 & -3.36581700 & 3.30663200\end{array}$

$\begin{array}{llll}0 & -2.13509800 & -2.81612300 & 2.18864500\end{array}$

$\begin{array}{llll}\text { C } & -4.03110600 & -2.22140000 & 0.88724500\end{array}$

$\mathrm{H} \quad-4.73212200 \quad-1.38853500 \quad 0.75744400$

$\mathrm{H} \quad-4.50550000 \quad-2.96993400 \quad 1.52694600$

$\begin{array}{llll}\text { C } & -3.57891000 & -2.75380100 & -0.45398400\end{array}$

$\begin{array}{llll}\text { C } & -2.37075100 & -3.40085800 & -2.87573200\end{array}$

$\begin{array}{llll}\text { C } & -4.23154800 & -3.61259800 & -1.33041700\end{array}$

$\begin{array}{llll}\text { C } & -2.32881100 & -2.23279900 & -0.79103600\end{array}$

$\begin{array}{llll}\text { C } & -1.71587100 & -2.53353800 & -1.99984400\end{array}$

$\begin{array}{llll}\text { C } & -3.61543500 & -3.93609600 & -2.54079400\end{array}$

$\mathrm{H} \quad-5.20193500 \quad-4.03017000 \quad-1.07704900$

$\mathrm{H} \quad-0.75132000 \quad-2.10637000 \quad-2.26715500$

$\mathrm{H} \quad-4.11113000 \quad-4.61177500 \quad-3.23131300$

$\mathrm{H} \quad-1.90896700 \quad-3.65832400 \quad-3.82370500$

$\begin{array}{llll}\text { C } & 2.52201100 & -2.13452700 & -2.04516000\end{array}$

$\mathrm{H} \quad 1.96292200 \quad-1.32750500 \quad-2.53678800$

$\mathrm{H} \quad 1.80293000 \quad-2.83998400 \quad-1.61611200$

$\mathrm{H} \quad 3.08608000 \quad-2.66025900 \quad-2.81882800$ 


\begin{tabular}{|c|c|c|c|}
\hline $\mathrm{C}$ & 3.49967700 & -0.10136400 & 2.54506700 \\
\hline$H$ & 2.53623200 & 0.41246800 & 2.49107500 \\
\hline $\mathrm{H}$ & 4.23713100 & 0.60300000 & 2.93740400 \\
\hline $\mathrm{H}$ & 3.39654400 & -0.92460000 & 3.25856100 \\
\hline $\mathrm{C}$ & 7.17544200 & -0.67604400 & -0.82489800 \\
\hline $\mathrm{H}$ & 7.37802700 & 0.37941100 & -1.04010800 \\
\hline $\mathrm{H}$ & 7.43142800 & -1.25437400 & -1.71624100 \\
\hline $\mathrm{H}$ & 7.84385200 & -0.97661600 & -0.01253600 \\
\hline $\mathrm{H}$ & 4.82822300 & 2.35058200 & -1.01357400 \\
\hline $\mathrm{C}$ & 3.76073200 & 2.29703900 & -0.81301000 \\
\hline $\mathrm{C}$ & 1.01624900 & 2.18515300 & -0.30232700 \\
\hline $\mathrm{C}$ & 3.24194200 & 2.88743200 & 0.34350800 \\
\hline $\mathrm{C}$ & 2.92930400 & 1.64340200 & -1.71816300 \\
\hline $\mathrm{C}$ & 1.53720400 & 1.61823600 & -1.50740000 \\
\hline $\mathrm{C}$ & 1.87160600 & 2.82393600 & 0.59767400 \\
\hline $\mathrm{H}$ & 3.89973000 & 3.39932200 & 1.03974500 \\
\hline $\mathrm{H}$ & 3.33078200 & 1.18645300 & -2.61879700 \\
\hline $\mathrm{H}$ & 1.46373800 & 3.28616600 & 1.49389500 \\
\hline $\mathrm{C}$ & -0.41715500 & 1.90540500 & -0.19394300 \\
\hline $\mathrm{C}$ & -0.98423800 & 1.51160000 & -1.36754900 \\
\hline $\mathrm{C}$ & -2.05891100 & 1.46160100 & -2.15113900 \\
\hline $\mathrm{H}$ & -2.21215500 & 0.66082700 & -2.86949200 \\
\hline 0 & 0.67260700 & 1.07594300 & -2.32414800 \\
\hline $\mathrm{C}$ & -1.10863600 & 1.80689000 & 1.11595800 \\
\hline $\mathrm{C}$ & -2.34818000 & 1.60895200 & 3.66923200 \\
\hline $\mathrm{C}$ & -0.40103200 & 1.40763700 & 2.26274200 \\
\hline $\mathrm{C}$ & -2.48346200 & 2.06736800 & 1.30335800 \\
\hline $\mathrm{C}$ & -3.08969100 & 1.98223200 & 2.55189900 \\
\hline $\mathrm{C}$ & -0.99620400 & 1.30896900 & 3.51711800 \\
\hline $\mathrm{H}$ & 0.64898600 & 1.15525400 & 2.14966300 \\
\hline $\mathrm{H}$ & -4.14917800 & 2.20703400 & 2.62438500 \\
\hline $\mathrm{H}$ & -0.40070600 & 0.99939300 & 4.37106000 \\
\hline $\mathrm{H}$ & -2.82546600 & 1.54952500 & 4.64225400 \\
\hline 0 & -3.32226600 & 2.29066000 & 0.23028600 \\
\hline $\mathrm{C}$ & -2.96599400 & 3.20967300 & -0.73231600 \\
\hline 0 & -2.61159700 & 4.32060000 & -0.45584000 \\
\hline $\mathrm{C}$ & -3.08181500 & 2.59225900 & -2.10630300 \\
\hline $\mathrm{H}$ & -4.08603300 & 2.15287700 & -2.17265700 \\
\hline $\mathrm{C}$ & -2.89244700 & 3.63468700 & -3.20356500 \\
\hline $\mathrm{H}$ & -1.90938300 & 4.10396600 & -3.10804000 \\
\hline $\mathrm{H}$ & -3.65787400 & 4.41609900 & -3.15140500 \\
\hline $\mathrm{H}$ & -2.95269800 & 3.15768000 & -4.18586000 \\
\hline
\end{tabular}

Zero-point correction= 0.663506 (Hartree/Particle)

Thermal correction to Energy= 0.701375 
Thermal correction to Enthalpy=

Thermal correction to Gibbs Free Energy=

Sum of electronic and zero-point Energies=

Sum of electronic and thermal Energies=

Sum of electronic and thermal Enthalpies=

Sum of electronic and thermal Free Energies=

$\mathrm{E}(\mathrm{RM} 062 \mathrm{X} / 6-311+\mathrm{G}(2 \mathrm{~d}, \mathrm{p}))=-1972.11430878$
0.702319

0.594652

$-1970.872920$

$-1970.835051$

$-1970.834107$

$-1970.941774$

\section{TS-QM}

\begin{tabular}{|c|c|c|c|}
\hline $\mathrm{C}$ & 1.76123800 & -0.56629800 & 7200 \\
\hline $\mathrm{C}$ & 0.53782300 & -1.05163400 & 0.61804900 \\
\hline $\mathrm{C}$ & 1.75944900 & 0.42849800 & -0.96687300 \\
\hline 0 & 0.72017900 & 0.92045700 & -1.44523200 \\
\hline $\mathrm{C}$ & 3.00175600 & -0.93242600 & 0.73133400 \\
\hline $\mathrm{H}$ & 2.97513000 & -1.63343800 & 1.56091300 \\
\hline $\mathrm{C}$ & 4.19960500 & -0.47002000 & 0.27324600 \\
\hline $\mathrm{H}$ & 5.12979100 & -0.79044400 & 0.7301350 \\
\hline $\mathrm{C}$ & 4.22162900 & 0.43598600 & -0.83336600 \\
\hline $\mathrm{H}$ & 5.18053100 & 0.79295300 & -1.2024450 \\
\hline $\mathrm{C}$ & 3.07237000 & 0.86297900 & -1.4236830 \\
\hline $\mathrm{H}$ & 3.08620600 & 1.56446500 & -2.2530890 \\
\hline $\mathrm{C}$ & 0.56328000 & -1.83496100 & 1.83074000 \\
\hline $\mathrm{H}$ & 0.57783400 & -3.11522000 & 3.7149310 \\
\hline $\mathrm{C}$ & 0.57514300 & -2.51298700 & 2.8295530 \\
\hline $\mathrm{C}$ & -0.66083600 & -1.31142400 & -0.22515200 \\
\hline $\mathrm{C}$ & -2.83245100 & -2.16719700 & -1.77642000 \\
\hline $\mathrm{C}$ & -0.47580300 & -1.77014500 & -1.53705000 \\
\hline $\mathrm{C}$ & -1.96973900 & -1.27469800 & 0.30823600 \\
\hline $\mathrm{C}$ & -3.04342400 & -1.70906400 & -0.48587300 \\
\hline $\mathrm{C}$ & -1.54183400 & -2.19395700 & -2.31478300 \\
\hline $\mathrm{H}$ & 0.53439700 & -1.80930500 & -1.93303500 \\
\hline $\mathrm{H}$ & -4.03990900 & -1.66618000 & -0.05687100 \\
\hline $\mathrm{H}$ & -1.37117800 & -2.55408200 & -3.32398200 \\
\hline $\mathrm{H}$ & -3.67779400 & -2.50197300 & -2.37121900 \\
\hline 0 & -2.23534300 & -0.80565000 & 1.53199900 \\
\hline $\mathrm{H}$ & -1.44889700 & -0.18653900 & 1.78585300 \\
\hline $\mathrm{H}$ & -0.60035900 & 1.83313100 & 0.76122300 \\
\hline 0 & -0.27650300 & 0.68777300 & 1.59616900 \\
\hline $\mathrm{H}$ & 0.35380100 & 0.80299000 & 2.322278 \\
\hline $\mathrm{N}$ & -1.17358500 & 2.65058000 & 0.26566900 \\
\hline $\mathrm{C}$ & -2.11074000 & 1.98401600 & -0.66603100 \\
\hline $\mathrm{H}$ & -2.71923800 & 2.74047500 & -1.16994600 \\
\hline $\mathrm{H}$ & -2.75127200 & 1.31638900 & -0.08601500 \\
\hline $\mathrm{H}$ & -1.52299200 & 1.40742100 & -1.38122100 \\
\hline
\end{tabular}




$\begin{array}{lrrr}\mathrm{C} & -0.28030900 & 3.59604500 & -0.43615300 \\ \mathrm{H} & 0.44916400 & 3.98285900 & 0.27841800 \\ \mathrm{H} & 0.22966100 & 3.05724600 & -1.23384800 \\ \mathrm{H} & -0.87650100 & 4.42072500 & -0.83726100 \\ \mathrm{C} & -1.90054300 & 3.31319500 & 1.36866600 \\ \mathrm{H} & -2.48326400 & 2.56063800 & 1.90223200 \\ \mathrm{H} & -2.56125600 & 4.08426100 & 0.96406500 \\ \mathrm{H} & -1.17509900 & 3.76348000 & 2.04863900\end{array}$

Zero-point correction $=$

0.354258 (Hartree/Particle)

Thermal correction to Energy=

0.376107

Thermal correction to Enthalpy=

0.377051

Thermal correction to Gibbs Free Energy=

0.304060

Sum of electronic and zero-point Energies=

$-978.091227$

Sum of electronic and thermal Energies=

$-978.069379$

Sum of electronic and thermal Enthalpies=

$-978.068434$

Sum of electronic and thermal Free Energies=

$-978.141426$

$\mathrm{E}(\mathrm{RM} 062 \mathrm{X} / 6-311+\mathrm{G}(2 \mathrm{~d}, \mathrm{p}))=-978.768396415$

water

$\begin{array}{lccc}0 & 0.00000000 & 0.11962100 & 0.00000000 \\ \mathrm{H} & 0.76087000 & -0.47822900 & 0.00000000 \\ \mathrm{H} & -0.76087000 & -0.47874300 & 0.00000000\end{array}$

Zero-point correction=

Thermal correction to Energy=

0.021314 (Hartree/Particle)

0.024149

Thermal correction to Enthalpy=

0.025093

Thermal correction to Gibbs Free Energy=

0.002995

Sum of electronic and zero-point Energies=

$-76.358850$

Sum of electronic and thermal Energies=

$-76.356015$

Sum of electronic and thermal Enthalpies=

$-76.355071$

Sum of electronic and thermal Free Energies=

$-76.377169$

$\mathrm{E}(\mathrm{RM} 062 \mathrm{X} / 6-311+\mathrm{G}(2 \mathrm{~d}, \mathrm{p}))=-76.4288823496$

\subsubsection{Origin of Enantioselectivity}

\section{TS1S}

$\begin{array}{rrrr}\mathrm{N} & 2.64620200 & 1.75833100 & 1.49050300 \\ \mathrm{C} & 3.00716500 & 0.51076000 & 1.56372400 \\ \mathrm{~N} & 2.38811600 & -0.24185700 & 0.60812200 \\ \mathrm{C} & 1.58476500 & 0.59168000 & -0.09887000 \\ \mathrm{~N} & 1.76554000 & 1.79792300 & 0.45370400 \\ \mathrm{C} & 1.13031600 & 3.05527200 & 0.16108800 \\ \mathrm{C} & -0.03950700 & 5.50325900 & -0.34929000 \\ \mathrm{C} & 0.00125500 & 3.40772700 & 0.89769500 \\ \mathrm{C} & 1.72179700 & 3.88292600 & -0.80063800\end{array}$




$\begin{array}{cccc}\mathrm{C} & 1.10908700 & 5.10630100 & -1.04686200 \\ \mathrm{C} & -0.57320400 & 4.65087000 & 0.61540600 \\ \mathrm{H} & 1.53517500 & 5.76927200 & -1.79646600 \\ \mathrm{H} & -1.46317400 & 4.95055300 & 1.16342700 \\ \mathrm{C} & 2.95373300 & 3.43646600 & -1.54036700 \\ \mathrm{H} & 3.29052100 & 4.21280600 & -2.23047100 \\ \mathrm{H} & 2.75729800 & 2.52548200 & -2.11978300 \\ \mathrm{H} & 3.77126400 & 3.21213100 & -0.84633600 \\ \mathrm{C} & -0.56653600 & 2.49543000 & 1.95047700 \\ \mathrm{H} & -0.06937700 & 2.66434400 & 2.91342900 \\ \mathrm{H} & -0.43486700 & 1.44388000 & 1.68327600 \\ \mathrm{H} & -1.63562400 & 2.68031700 & 2.08366900 \\ \mathrm{C} & -0.67992600 & 6.83266400 & -0.64858300 \\ \mathrm{H} & 0.03662000 & 7.65014400 & -0.51798200 \\ \mathrm{H} & -1.53426900 & 7.01757000 & 0.00706000 \\ \mathrm{H} & -1.03129300 & 6.87096600 & -1.68512400 \\ \mathrm{C} & 0.70549800 & 0.14209600 & -1.21728100 \\ \mathrm{C} & 2.61948000 & -1.69030000 & 0.46786000 \\ \mathrm{H} & 1.64386700 & -2.16913700 & 0.39000000 \\ \mathrm{C} & 3.37937400 & -2.22780600 & 1.70325700 \\ \mathrm{H} & -1.04206400 & -0.35990800 & -3.25942500 \\ \mathrm{H} & 2.70929400 & -2.39267200 & 2.55618600 \\ \mathrm{C} & 3.92021000 & -0.11842000 & 2.57131200 \\ \mathrm{H} & -1.09425400 & 1.30051000 & -3.87005800 \\ \mathrm{H} & 3.36495200 & -0.27427700 & 3.50748100 \\ \mathrm{H} & 4.76454900 & 0.54369400 & 2.77118100 \\ \mathrm{H} & 4.41993900 & -1.33089400 & 2.06695200 \\ \mathrm{C} & 4.05272100 & -3.49597100 & 1.17522300 \\ \mathrm{H} & 4.94990800 & -3.72765200 & 1.75467400 \\ \mathrm{H} & 3.36432800 & -4.34518100 & 1.25408000 \\ \mathrm{C} & 3.52180300 & -2.08749700 & -0.68275000 \\ \mathrm{C} & 5.32340500 & -3.22236500 & -2.45073100 \\ \mathrm{C} & 3.60628600 & -1.57899600 & -1.97208400 \\ \mathrm{C} & 4.32930500 & -3.15064000 & -0.27012400 \\ \mathrm{H} & 5.23181500 & -3.72938000 & -1.15348200 \\ \mathrm{H} & 4.52253300 & -2.15396400 & -2.85469900 \\ \mathrm{H} & 2.98451700 & -0.74893800 & -2.29461000 \\ \mathrm{H} & 4.66521900 & -4.55328000 & -0.83648000 \\ \mathrm{H} & -0.5343430000 & -1.76592000 & -3.86481000 \\ \mathrm{H} & -1.09506900 & -1.30168100 \\ \mathrm{H} & & & \\ \mathrm{H} & & & \\ \mathrm{H} & & & \\ \mathrm{H} & & & \\ \mathrm{H} & & & \end{array}$




\begin{tabular}{|c|c|c|c|}
\hline $\mathrm{H}$ & 0.40082200 & 2.07723700 & -2.04048000 \\
\hline $\mathrm{H}$ & -1.59002300 & 2.41355400 & 1.05504300 \\
\hline $\mathrm{C}$ & -1.51527900 & 1.32682400 & 1.09934900 \\
\hline $\mathrm{C}$ & -2.32161500 & 0.44106200 & 0.65286400 \\
\hline $\mathrm{C}$ & -2.79359500 & -0.86379000 & 0.24576700 \\
\hline $\mathrm{C}$ & -4.13981500 & -1.22299900 & 0.88882900 \\
\hline $\mathrm{C}$ & -6.67198200 & -1.86262200 & 1.93038900 \\
\hline $\mathrm{C}$ & -4.93623400 & -0.25511900 & 1.50186700 \\
\hline $\mathrm{C}$ & -4.63504800 & -2.54182200 & 0.81279000 \\
\hline $\mathrm{C}$ & -5.89718100 & -2.84723300 & 1.32949600 \\
\hline $\mathrm{C}$ & -6.18957000 & -0.55930600 & 2.02708500 \\
\hline $\mathrm{H}$ & -4.55416800 & 0.76013100 & 1.54728700 \\
\hline $\mathrm{H}$ & -6.24570700 & -3.87262000 & 1.25140100 \\
\hline $\mathrm{H}$ & -6.78388300 & 0.21685500 & 2.49866700 \\
\hline $\mathrm{H}$ & -7.65065700 & -2.11769100 & 2.32683300 \\
\hline $\mathrm{C}$ & -2.85719500 & -0.83828800 & 1.29369400 \\
\hline $\mathrm{C}$ & -2.90190900 & -0.60536300 & 4.09343600 \\
\hline $\mathrm{C}$ & -4.06196800 & -0.71829600 & 1.98682300 \\
\hline $\mathrm{C}$ & -1.65905400 & -0.85474500 & 2.03563900 \\
\hline $\mathrm{C}$ & -1.68930400 & -0.73460100 & 3.42563100 \\
\hline $\mathrm{C}$ & -4.09510400 & -0.60370400 & 3.37433800 \\
\hline $\mathrm{H}$ & -4.99069700 & -0.70387300 & 1.42371500 \\
\hline $\mathrm{H}$ & -0.74512700 & -0.74706900 & 3.96236000 \\
\hline $\mathrm{H}$ & -5.04726100 & -0.51098700 & 3.88699800 \\
\hline $\mathrm{H}$ & -2.91254000 & -0.51226600 & 5.17545800 \\
\hline 0 & -1.86891900 & -2.00660400 & .53543000 \\
\hline $\mathrm{H}$ & -1.32246800 & -1.75367400 & 1.30370200 \\
\hline 0 & -3.94510100 & -3.55164100 & .23239900 \\
\hline $\mathrm{H}$ & -3.00283300 & -3.27911200 & 0.21579400 \\
\hline 0 & -0.43449000 & -0.94449500 & 1.44667500 \\
\hline $\mathrm{H}$ & -0.56561200 & -1.31962600 & 0.54835700 \\
\hline Zer & point correction & & 0.688895 (Hartree/Particle) \\
\hline The & hal correction to & nergy= & 0.729272 \\
\hline The & hal correction to & nthalpy $=$ & 0.730217 \\
\hline The & hal correction to & ibbs Free Energy= & 0.616666 \\
\hline Sur & f electronic and & ero-point Energies= & -2047.194044 \\
\hline Sur & f electronic and & nermal Energies= & -2047.153666 \\
\hline Sur & f electronic and & nermal Enthalpies= & -2047.152722 \\
\hline Sur & f electronic and & nermal Free Energies= & -2047.266272 \\
\hline $\mathrm{E}(\mathrm{F}$ & $062 X / 6-311+G$ & $d, p))=-2048.50458$ & 58436 \\
\hline \multicolumn{4}{|c|}{ TS1-S2 } \\
\hline $\mathrm{N}$ & -3.95583600 & 0.96319300 & .01447100 \\
\hline $\mathrm{C}$ & -4.05583100 & -0.30422300 & 27709400 \\
\hline
\end{tabular}




\begin{tabular}{|c|c|c|c|}
\hline $\mathrm{N}$ & -2.66852300 & 1.27955700 & -0.32500000 \\
\hline $\mathrm{N}$ & -2.86976600 & -0.81311900 & -0.73778600 \\
\hline $\mathrm{C}$ & -1.99002900 & 0.22199100 & -0.79574200 \\
\hline $\mathrm{C}$ & -2.22857200 & 2.63030100 & -0.07826600 \\
\hline $\mathrm{C}$ & -1.49167000 & 5.24039300 & 0.45363200 \\
\hline $\mathrm{C}$ & -2.67234900 & 3.62997200 & -0.94197700 \\
\hline $\mathrm{C}$ & -1.43764000 & 2.87620900 & 1.04905100 \\
\hline $\mathrm{C}$ & -1.08046200 & 4.19899900 & 1.29195600 \\
\hline $\mathrm{C}$ & -2.28373500 & 4.93975400 & -0.65365500 \\
\hline $\mathrm{H}$ & -0.46001000 & 4.42301300 & 2.15717300 \\
\hline $\mathrm{H}$ & -2.60954100 & 5.74007800 & -1.31361700 \\
\hline $\mathrm{C}$ & -0.96063100 & 1.75949600 & 1.93583300 \\
\hline $\mathrm{H}$ & -1.76125500 & 1.04415200 & 2.15550900 \\
\hline $\mathrm{H}$ & -0.58775600 & 2.15570500 & 2.88294400 \\
\hline $\mathrm{H}$ & -0.14220000 & 1.21880100 & 1.44376500 \\
\hline $\mathrm{C}$ & -3.52598300 & 3.30872800 & -2.13939700 \\
\hline $\mathrm{H}$ & -4.49948300 & 2.90768100 & -1.83798200 \\
\hline $\mathrm{H}$ & -3.04521600 & 2.56108600 & -2.78012000 \\
\hline $\mathrm{H}$ & -3.69680000 & 4.20710000 & -2.73651700 \\
\hline $\mathrm{C}$ & -1.07658700 & 6.65706800 & 0.75091800 \\
\hline $\mathrm{H}$ & -1.49490700 & 7.35542400 & 0.02166200 \\
\hline $\mathrm{H}$ & 0.01392600 & 6.75532300 & 0.73100500 \\
\hline $\mathrm{H}$ & -1.41175300 & 6.96244200 & 1.74777600 \\
\hline $\mathrm{C}$ & -2.65962200 & -2.26538300 & -0.84320000 \\
\hline $\mathrm{H}$ & -2.06895900 & -2.47673400 & -1.73730300 \\
\hline $\mathrm{C}$ & -4.01396500 & -3.00927900 & -0.94513400 \\
\hline $\mathrm{H}$ & -4.45183600 & -2.94484800 & -1.94857900 \\
\hline $\mathrm{C}$ & -5.28020100 & -1.16464300 & -0.20541700 \\
\hline $\mathrm{H}$ & -5.91755100 & -0.83643500 & 0.61698800 \\
\hline $\mathrm{H}$ & -5.83750300 & -1.06085000 & -1.14787000 \\
\hline 0 & -4.91879600 & -2.50363000 & 0.02488500 \\
\hline $\mathrm{C}$ & -2.04004200 & -2.89933500 & 0.38643300 \\
\hline $\mathrm{C}$ & -1.20471800 & -4.49044000 & 2.48189100 \\
\hline $\mathrm{C}$ & -1.08493300 & -2.39995600 & 1.25842700 \\
\hline $\mathrm{C}$ & -2.59497800 & -4.17055000 & 0.55640600 \\
\hline $\mathrm{C}$ & -2.17340200 & -4.97959000 & 1.60282900 \\
\hline $\mathrm{C}$ & -0.67173300 & -3.21217400 & 2.31730700 \\
\hline $\mathrm{H}$ & -0.66266500 & -1.40544700 & 1.12880200 \\
\hline $\mathrm{H}$ & -2.60241300 & -5.96712700 & 1.74714300 \\
\hline $\mathrm{H}$ & 0.06951500 & -2.84328200 & 3.01981600 \\
\hline $\mathrm{H}$ & -0.87244600 & -5.10706600 & 3.31160200 \\
\hline $\mathrm{C}$ & -3.65281200 & -4.42652800 & -0.49408800 \\
\hline $\mathrm{H}$ & -4.53472900 & -4.94810600 & -0.11407000 \\
\hline $\mathrm{H}$ & -3.25070000 & -4.99847000 & -1.33841600 \\
\hline
\end{tabular}




\begin{tabular}{|c|c|c|c|}
\hline $\mathrm{C}$ & -0.63679000 & 0.34874800 & -1.41226700 \\
\hline 0 & 0.05970200 & 1.30997300 & -1.01013100 \\
\hline $\mathrm{C}$ & -0.20253900 & -0.61557800 & -2.36654000 \\
\hline $\mathrm{H}$ & -0.98350000 & -1.28353300 & -2.72204600 \\
\hline $\mathrm{C}$ & 0.66007300 & -0.10798800 & -3.51028100 \\
\hline $\mathrm{H}$ & 1.31010200 & 0.70493000 & -3.18377100 \\
\hline $\mathrm{H}$ & 0.02880500 & 0.28051700 & -4.31740600 \\
\hline $\mathrm{H}$ & 1.28207100 & -0.90946600 & -3.91788100 \\
\hline $\mathrm{H}$ & 0.35258700 & -2.63918300 & -1.41114600 \\
\hline $\mathrm{C}$ & 0.86151600 & -1.68073200 & -1.33546900 \\
\hline $\mathrm{C}$ & 1.92673400 & -1.33331000 & -0.72802000 \\
\hline $\mathrm{C}$ & 2.82679400 & -0.23329900 & -0.32918300 \\
\hline 0 & 2.68087900 & 0.95489000 & -1.17906100 \\
\hline $\mathrm{H}$ & 1.70789200 & 1.16746800 & -1.16893700 \\
\hline $\mathrm{C}$ & 4.28993000 & -0.66416400 & -0.53839900 \\
\hline $\mathrm{C}$ & 6.89420000 & -1.60599900 & -1.00207800 \\
\hline $\mathrm{C}$ & 5.14268900 & -0.96890400 & 0.52087900 \\
\hline $\mathrm{C}$ & 4.77040200 & -0.82825300 & -1.85303100 \\
\hline $\mathrm{C}$ & 6.06528800 & -1.29727700 & -2.07585000 \\
\hline $\mathrm{C}$ & 6.43772800 & -1.43683100 & 0.30196400 \\
\hline $\mathrm{H}$ & 4.78357300 & -0.84620200 & 1.53844400 \\
\hline $\mathrm{H}$ & 6.40246100 & -1.40958800 & -3.10186400 \\
\hline $\mathrm{H}$ & 7.07979500 & -1.66735700 & 1.14612100 \\
\hline $\mathrm{H}$ & 7.90027000 & -1.97056600 & -1.18866000 \\
\hline $\mathrm{C}$ & 2.54651800 & 0.17778100 & 1.12157000 \\
\hline $\mathrm{C}$ & 2.08335000 & 0.91424600 & 3.79551500 \\
\hline $\mathrm{C}$ & 2.93631800 & 1.44927100 & 1.59211600 \\
\hline $\mathrm{C}$ & 1.95221800 & -0.70715400 & 2.02452800 \\
\hline $\mathrm{C}$ & 1.71224900 & -0.35370600 & 3.35005600 \\
\hline $\mathrm{C}$ & 2.68817800 & 1.80825400 & 2.92146000 \\
\hline $\mathrm{H}$ & 1.68933600 & -1.69848900 & 1.66531800 \\
\hline $\mathrm{H}$ & 1.25276700 & -1.06369000 & 4.03082400 \\
\hline $\mathrm{H}$ & 2.99142500 & 2.79935500 & 3.24511700 \\
\hline $\mathrm{H}$ & 1.90510000 & 1.20816600 & 4.82610300 \\
\hline 0 & 4.00450600 & -0.56026000 & -2.93979000 \\
\hline $\mathrm{H}$ & 3.27468200 & 0.01798500 & -2.64061600 \\
\hline 0 & 3.57266100 & 2.35680000 & 0.81893100 \\
\hline $\mathrm{H}$ & 3.46441800 & 2.06211300 & -0.11327000 \\
\hline \multicolumn{3}{|c|}{ Zero-point correction $=$} & 0.689642 (Hartree/Particle) \\
\hline \multicolumn{3}{|c|}{ Thermal correction to Energy= } & 0.729614 \\
\hline \multicolumn{3}{|c|}{ Thermal correction to Enthalpy= } & 0.730558 \\
\hline \multicolumn{3}{|c|}{ Thermal correction to Gibbs Free Energy= } & 0.618605 \\
\hline \multirow{2}{*}{\multicolumn{3}{|c|}{$\begin{array}{l}\text { Sum of electronic and zero-point Energies= } \\
\text { Sum of electronic and thermal Energies }=\end{array}$}} & -2047.186370 \\
\hline & & & -2047.146398 \\
\hline
\end{tabular}


Sum of electronic and thermal Enthalpies=

$-2047.145454$

Sum of electronic and thermal Free Energies=

$-2047.257407$

$\mathrm{E}\left(\mathrm{RM} 062 \mathrm{X} / 6-311+\mathrm{G}^{* *}\right)=-2048.49981223$

TS1-R

$\begin{array}{lrrr}\mathrm{C} & 3.20143400 & 0.97244500 & 1.51384700 \\ \mathrm{~N} & 2.68416500 & 2.12432700 & 1.20408800 \\ \mathrm{~N} & 1.71409600 & 1.83439000 & 0.29629900 \\ \mathrm{~N} & 2.60605800 & -0.04057600 & 0.81373500 \\ \mathrm{C} & 1.63234400 & 0.51915900 & 0.05118200 \\ \mathrm{C} & 1.03136700 & 2.94927100 & -0.32194700 \\ \mathrm{C} & -0.17026800 & 5.17307700 & -1.43809200 \\ \mathrm{C} & 1.58157600 & 3.48020200 & -1.48618300 \\ \mathrm{C} & -0.09495500 & 3.47703700 & 0.31105500 \\ \mathrm{C} & -0.68119700 & 4.59753400 & -0.27021000 \\ \mathrm{C} & 0.95531900 & 4.60308900 & -2.03115100 \\ \mathrm{H} & -1.56440100 & 5.02845500 & 0.19659500 \\ \mathrm{H} & 1.36222000 & 5.03963600 & -2.94012800 \\ \mathrm{C} & 3.05193600 & -1.43595200 & 0.93983700 \\ \mathrm{H} & 2.16129900 & -2.06513400 & 1.00965600 \\ \mathrm{C} & 4.22816400 & 0.68612400 & 2.56726700 \\ \mathrm{H} & 3.73406300 & 0.70427400 & 3.54933700 \\ \mathrm{H} & 5.00243700 & 1.45529800 & 2.55071300 \\ \mathrm{C} & 3.89919800 & -1.61758100 & 2.22254200 \\ \mathrm{H} & 3.27965700 & -1.67220000 & 3.12577200 \\ \mathrm{O} & 4.83786400 & -0.55753000 & 2.32745900 \\ \mathrm{C} & 4.70152800 & -2.88543100 & 1.92356800 \\ \mathrm{H} & 4.10867600 & -3.77233000 & 2.17569900 \\ \mathrm{H} & 5.62279100 & -2.90957300 & 2.51069300 \\ \mathrm{C} & 4.00614500 & -1.90701200 & -0.14411100 \\ \mathrm{C} & 5.95484100 & -3.08572800 & -1.71421800 \\ \mathrm{C} & 4.93272700 & -2.78001100 & 0.43327000 \\ \mathrm{C} & 4.05653000 & -1.59525800 & -1.49689400 \\ \mathrm{C} & 5.04442300 & -2.19455700 & -2.28109800 \\ \mathrm{C} & 5.90810600 & -3.38174900 & -0.35028700 \\ \mathrm{H} & 3.35377300 & -0.89493400 & -1.94187000 \\ \mathrm{H} & 5.10446300 & -1.95953600 & -3.33909700 \\ \mathrm{H} & 6.63461000 & -4.05674900 & 0.09317700 \\ \mathrm{H} & -0.71815600 & -3.54231000 & -2.33694500 \\ \mathrm{H} & -1.099762000 & 2.81779300 & 1.53017100 \\ \mathrm{H} & 1.84477000 & 1.25719100 \\ \mathrm{H} & 3.78417900 & 2.85505000 & -2.13977300\end{array}$




\begin{tabular}{|c|c|c|c|}
\hline $\mathrm{H}$ & 2.54647800 & 1.85438000 & -2.52073200 \\
\hline $\mathrm{H}$ & 3.61849400 & 2.75503600 & -1.43713400 \\
\hline $\mathrm{H}$ & 3.12041300 & 3.46193000 & -2.98321600 \\
\hline $\mathrm{C}$ & -0.83533900 & 6.38460400 & -2.03698700 \\
\hline $\mathrm{H}$ & -1.87512100 & 6.16655200 & -2.30245100 \\
\hline $\mathrm{H}$ & -0.31615200 & 6.71777200 & -2.93921300 \\
\hline $\mathrm{H}$ & -0.85005400 & 7.21559800 & -1.32403300 \\
\hline $\mathrm{C}$ & 0.60497500 & -0.06826800 & -0.86173500 \\
\hline $\mathrm{C}$ & 0.61388400 & -1.45463000 & -1.18935000 \\
\hline $\mathrm{H}$ & 1.55663600 & -1.96258900 & -1.01179200 \\
\hline $\mathrm{C}$ & 0.00215900 & -1.83206600 & -2.52726000 \\
\hline $\mathrm{H}$ & 0.74340400 & -1.72280300 & -3.32778200 \\
\hline $\mathrm{H}$ & -0.83766700 & -1.17670700 & -2.76579600 \\
\hline $\mathrm{H}$ & -0.35005000 & -2.86655000 & -2.52524800 \\
\hline 0 & -0.32307600 & 0.72071600 & -1.16418000 \\
\hline $\mathrm{H}$ & 0.19763200 & -2.88406200 & 0.56713900 \\
\hline $\mathrm{C}$ & -0.46796400 & -2.16545400 & 0.09668200 \\
\hline $\mathrm{C}$ & -1.69972800 & -1.90985900 & 0.30132200 \\
\hline $\mathrm{C}$ & -2.87919700 & -1.09355900 & -0.02348600 \\
\hline $\mathrm{C}$ & -3.13520200 & -0.05969300 & 1.07942100 \\
\hline $\mathrm{C}$ & -3.73782700 & 1.76873000 & 3.12735600 \\
\hline $\mathrm{C}$ & -2.61746400 & -0.21817000 & 2.36671900 \\
\hline $\mathrm{C}$ & -3.97572100 & 1.04533000 & 0.82851700 \\
\hline $\mathrm{C}$ & -4.26401000 & 1.95102400 & 1.85444000 \\
\hline $\mathrm{C}$ & -2.90431500 & 0.68248200 & 3.38931600 \\
\hline $\mathrm{H}$ & -1.98768300 & -1.08103400 & 2.56167200 \\
\hline $\mathrm{H}$ & -4.91205500 & 2.79158000 & 1.62543700 \\
\hline $\mathrm{H}$ & -2.49021900 & 0.52956500 & 4.38090800 \\
\hline $\mathrm{H}$ & -3.97817900 & 2.47859800 & 3.91383300 \\
\hline $\mathrm{C}$ & -4.09666600 & -2.02039300 & -0.22132400 \\
\hline $\mathrm{C}$ & -6.19309700 & -3.86372700 & -0.54602300 \\
\hline $\mathrm{C}$ & -5.09570300 & -2.15302800 & 0.74268400 \\
\hline $\mathrm{C}$ & -4.16915600 & -2.83100600 & -1.37176600 \\
\hline $\mathrm{C}$ & -5.21452200 & -3.74290500 & -1.52640300 \\
\hline $\mathrm{C}$ & -6.14046500 & -3.06294500 & 0.59167500 \\
\hline $\mathrm{H}$ & -5.05131700 & -1.53617700 & 1.63535300 \\
\hline $\mathrm{H}$ & -5.23777800 & -4.34767000 & -2.42790600 \\
\hline $\mathrm{H}$ & -6.90316000 & -3.14345900 & 1.35973100 \\
\hline $\mathrm{H}$ & -6.99992300 & -4.57889000 & -0.67790800 \\
\hline 0 & -2.75472500 & -0.38261000 & -1.30535700 \\
\hline $\mathrm{H}$ & -1.87107600 & 0.07261900 & -1.28896300 \\
\hline 0 & -4.55225700 & 1.26752000 & -0.37348800 \\
\hline $\mathrm{H}$ & -4.07261900 & 0.71165600 & -1.02754900 \\
\hline 0 & -3.23710900 & -2.77941900 & -2.35465700 \\
\hline
\end{tabular}




$\begin{array}{lc}\mathrm{H}-2.75811600 \quad-1.93254700 \quad-2.24813000 \\ \text { Zero-point correction= } & 0.689529 \text { (Hartree/Particle) } \\ \text { Thermal correction to Energy= } & 0.729700 \\ \text { Thermal correction to Enthalpy= } & 0.730644 \\ \text { Thermal correction to Gibbs Free Energy= } & 0.616827 \\ \text { Sum of electronic and zero-point Energies }= & -2047.186833 \\ \text { Sum of electronic and thermal Energies= } & -2047.146662 \\ \text { Sum of electronic and thermal Enthalpies }= & -2047.145718 \\ \text { Sum of electronic and thermal Free Energies }= & -2047.259535\end{array}$

$\mathrm{E}(\mathrm{RM} 062 \mathrm{X} / 6-311+\mathrm{G}(2 \mathrm{~d}, \mathrm{p}))=-2048.50125334$

TS1-R2

$\begin{array}{lrrr}\mathrm{C} & 3.32707300 & 1.49361000 & 1.31992800 \\ \mathrm{~N} & 2.58279100 & 2.49655800 & 0.96075500 \\ \mathrm{~N} & 1.64168900 & 1.95229600 & 0.14508300 \\ \mathrm{~N} & 2.89192900 & 0.32510700 & 0.75458000 \\ \mathrm{C} & 1.80215000 & 0.63056100 & 0.00172400 \\ \mathrm{C} & 0.63247600 & 2.83583900 & -0.39134800 \\ \mathrm{C} & -1.24889600 & 4.60955700 & -1.35963400 \\ \mathrm{C} & -0.57748200 & 2.95058500 & 0.29535800 \\ \mathrm{C} & 0.94628200 & 3.56768000 & -1.53278700 \\ \mathrm{C} & -0.02030200 & 4.45826400 & -2.00225600 \\ \mathrm{C} & -1.51094400 & 3.85046600 & -0.21439100 \\ \mathrm{H} & 0.19447800 & 5.04162400 & -2.89447600 \\ \mathrm{H} & -2.46744700 & 3.95353400 & 0.29235400 \\ \mathrm{C} & -0.85700800 & 2.11999200 & 1.51753100 \\ \mathrm{H} & -0.96197900 & 1.05904600 & 1.25486300 \\ \mathrm{H} & -1.78279600 & 2.44172400 & 1.99821500 \\ \mathrm{H} & -0.04259300 & 2.19798200 & 2.24672800 \\ \mathrm{C} & 2.25845500 & 3.37150600 & -2.24087800 \\ \mathrm{H} & 3.10689000 & 3.52219100 & -1.56477800 \\ \mathrm{H} & 2.35468300 & 4.07113200 & -3.07394200 \\ \mathrm{H} & 2.32972200 & 2.35351300 & -2.64178200 \\ \mathrm{C} & -2.28690900 & 5.56694200 & -1.88419900 \\ \mathrm{H} & -1.91566700 & 6.11928400 & -2.75113200 \\ \mathrm{H} & -2.57789200 & 6.29068500 & -1.11586700 \\ \mathrm{H} & -3.19356000 & 5.03105500 & -2.18465000 \\ \mathrm{C} & 3.56492700 & -0.96071800 & 1.00784800 \\ \mathrm{H} & 2.79683100 & -1.68462600 & 1.29119900 \\ \mathrm{C} & 4.55997200 & -0.82288300 & 2.18797900 \\ \mathrm{H} & 4.05023900 & -0.85443200 & 3.15871000 \\ \mathrm{C} & 4.48796600 & 1.51505200 & 2.26768400 \\ \mathrm{H} & 5.09389100 & 2.40466500 & 2.08835100 \\ \mathrm{H} & 4.10941700 & 1.54878500 & 3.29921000 \\ & & & \end{array}$




\begin{tabular}{|c|c|c|c|}
\hline 0 & 5.29481400 & 0.38291400 & 2.05872600 \\
\hline $\mathrm{C}$ & 5.53939300 & -1.97386500 & 1.95455100 \\
\hline $\mathrm{H}$ & 6.51131900 & -1.74956400 & 2.40089100 \\
\hline $\mathrm{H}$ & 5.15468500 & -2.89140100 & 2.41430600 \\
\hline $\mathrm{C}$ & 4.44748000 & -1.47393700 & -0.11504600 \\
\hline $\mathrm{C}$ & 6.35631300 & -2.63529900 & -1.74981400 \\
\hline $\mathrm{C}$ & 4.27288200 & -1.42413600 & -1.49274800 \\
\hline $\mathrm{C}$ & 5.57073300 & -2.08660500 & 0.44824100 \\
\hline $\mathrm{C}$ & 6.52929300 & -2.67635000 & -0.36527600 \\
\hline $\mathrm{C}$ & 5.24213300 & -2.01019800 & -2.30894100 \\
\hline $\mathrm{H}$ & 3.40889500 & -0.93670700 & -1.93659300 \\
\hline $\mathrm{H}$ & 7.40662600 & -3.14802300 & 0.06833700 \\
\hline $\mathrm{H}$ & 5.12604300 & -1.97494400 & -3.38755700 \\
\hline $\mathrm{H}$ & 7.10215900 & -3.08451300 & -2.39855600 \\
\hline $\mathrm{C}$ & 0.90493900 & -0.20768700 & -0.84970100 \\
\hline 0 & 0.06239600 & 0.42822400 & -1.52958700 \\
\hline $\mathrm{C}$ & 0.88263200 & -1.61946600 & -0.69704700 \\
\hline $\mathrm{H}$ & 1.80491700 & -2.08349400 & -0.36406900 \\
\hline $\mathrm{C}$ & 0.18440700 & -2.38506800 & -1.79969200 \\
\hline $\mathrm{H}$ & 0.10736800 & -3.44461600 & -1.54106100 \\
\hline $\mathrm{H}$ & 0.73344500 & -2.29745300 & -2.74460900 \\
\hline $\mathrm{H}$ & -0.82628300 & -2.00094400 & -1.96272900 \\
\hline $\mathrm{C}$ & -1.43528000 & -1.56267000 & 0.82111900 \\
\hline $\mathrm{H}$ & 0.51387700 & -2.15599400 & 1.53156800 \\
\hline $\mathrm{C}$ & -0.18054800 & -1.78183500 & 0.78361400 \\
\hline $\mathrm{C}$ & -2.66392800 & -1.08085500 & 0.18938700 \\
\hline $\mathrm{C}$ & -3.37250900 & -2.22955000 & -0.55487900 \\
\hline $\mathrm{C}$ & -4.79266000 & -4.28867300 & -1.83679700 \\
\hline $\mathrm{C}$ & -3.02648100 & -3.56948500 & -0.37008100 \\
\hline $\mathrm{C}$ & -4.44541300 & -1.93165600 & -1.41820800 \\
\hline $\mathrm{C}$ & -5.14724300 & -2.96194800 & -2.04820500 \\
\hline $\mathrm{C}$ & -3.72205000 & -4.59793500 & -1.00085700 \\
\hline $\mathrm{H}$ & -2.19777500 & -3.80359200 & 0.29137900 \\
\hline $\mathrm{H}$ & -5.96838900 & -2.69315300 & -2.70565500 \\
\hline $\mathrm{H}$ & -3.43110000 & -5.63031900 & -0.83473600 \\
\hline $\mathrm{H}$ & -5.34847700 & -5.07879000 & -2.33329100 \\
\hline $\mathrm{C}$ & -3.63066400 & -0.52802100 & 1.27657000 \\
\hline $\mathrm{C}$ & -5.41454600 & 0.22469300 & 3.32739100 \\
\hline $\mathrm{C}$ & -4.03827800 & -1.42973900 & 2.26791800 \\
\hline $\mathrm{C}$ & -4.13695400 & 0.78339100 & 1.33870900 \\
\hline $\mathrm{C}$ & -5.02326800 & 1.14111800 & 2.36626700 \\
\hline $\mathrm{C}$ & -4.91612600 & -1.07891700 & 3.28265300 \\
\hline $\mathrm{H}$ & -3.63958700 & -2.44039800 & 2.22626700 \\
\hline $\mathrm{H}$ & -5.38862600 & 2.16363300 & 2.37833400 \\
\hline
\end{tabular}




$\begin{array}{lccc}\mathrm{H} & -5.20772300 & -1.81074800 & 4.02911100 \\ \mathrm{H} & -6.10232200 & 0.52623000 & 4.11209800 \\ 0 & -2.45572800 & 0.00857200 & -0.76869000 \\ \mathrm{H} & -1.52831400 & 0.00148800 & -1.11787800 \\ 0 & -4.85832200 & -0.66283000 & -1.66000100 \\ \mathrm{H} & -4.10354200 & -0.07075500 & -1.47444000 \\ 0 & -3.82819400 & 1.77499300 & 0.46469700 \\ \mathrm{H} & -3.12809200 & 1.42462500 & -0.12834000\end{array}$

Zero-point correction= 0.688901 (Hartree/Particle)

Thermal correction to Energy= 0.729080

Thermal correction to Enthalpy= 0.730024

Thermal correction to Gibbs Free Energy= 0.616354

Sum of electronic and zero-point Energies= $-2047.186343$

Sum of electronic and thermal Energies= $-2047.146164$

Sum of electronic and thermal Enthalpies= $-2047.145220$

Sum of electronic and thermal Free Energies= $-2047.258890$

TS1-R3

$\begin{array}{lrrr}\mathrm{N} & 3.37071100 & -0.41950000 & 1.46897900 \\ \mathrm{C} & 2.62292100 & -1.48217400 & 1.39165000 \\ \mathrm{~N} & 2.85421000 & 0.42325700 & 0.53476200 \\ \mathrm{~N} & 1.67097900 & -1.34586100 & 0.41843300 \\ \mathrm{C} & 1.80856500 & -0.10763300 & -0.09935400 \\ \mathrm{C} & 3.47812400 & 1.69978500 & 0.30394600 \\ \mathrm{C} & 4.73910100 & 4.12621400 & -0.08244000 \\ \mathrm{C} & 3.23489900 & 2.72275500 & 1.22388200 \\ \mathrm{C} & 4.31571000 & 1.83683800 & -0.80186500 \\ \mathrm{C} & 4.93818800 & 3.07434500 & -0.97642700 \\ \mathrm{C} & 3.88241700 & 3.93545300 & 1.00684100 \\ \mathrm{H} & 5.59741200 & 3.21344600 & -1.82983500 \\ \mathrm{H} & 3.70876400 & 4.75416600 & 1.70145700 \\ \mathrm{C} & 4.52944100 & 0.70479400 & -1.77095100 \\ \mathrm{H} & 4.78151100 & -0.22545200 & -1.24994800 \\ \mathrm{H} & 5.34269000 & 0.94175600 & -2.46027500 \\ \mathrm{H} & 3.62661000 & 0.51896100 & -2.36623600 \\ \mathrm{C} & 2.29495000 & 2.51145200 & 2.37701700 \\ \mathrm{H} & 2.68156400 & 1.76014900 & 3.07421500 \\ \mathrm{H} & 1.32259000 & 2.15970700 & 2.01484000 \\ \mathrm{H} & 2.14636800 & 3.44399100 & 2.92568700 \\ \mathrm{C} & 5.44312600 & 5.44379200 & -0.27230700 \\ \mathrm{H} & 6.26259900 & 5.55253500 & 0.44699900 \\ \mathrm{H} & 4.75845200 & 6.28255300 & -0.11488400 \\ \mathrm{H} & 5.86550700 & 5.52653800 & -1.27690600\end{array}$




\begin{tabular}{|c|c|c|c|}
\hline $\mathrm{C}$ & 0.61746500 & -2.33950200 & 0.20774900 \\
\hline $\mathrm{H}$ & -0.31130600 & -1.78599600 & 0.03244900 \\
\hline $\mathrm{C}$ & 0.48474600 & -3.22623400 & 1.46203300 \\
\hline $\mathrm{H}$ & -0.00379000 & -2.70694300 & 2.29538400 \\
\hline $\mathrm{C}$ & 2.62078500 & -2.66478900 & 2.31794000 \\
\hline $\mathrm{H}$ & 3.62792100 & -3.07730100 & 2.40532600 \\
\hline $\mathrm{H}$ & 2.30103100 & -2.31673200 & 3.31051200 \\
\hline 0 & 1.77932300 & -3.68360300 & 1.83870100 \\
\hline $\mathrm{C}$ & 0.86589200 & -3.37118700 & -0.87573100 \\
\hline $\mathrm{C}$ & 0.95715400 & -5.58482800 & -2.53633800 \\
\hline $\mathrm{C}$ & 1.54642100 & -3.27830500 & -2.08085000 \\
\hline $\mathrm{C}$ & 0.27526100 & -4.57380000 & -0.47122000 \\
\hline $\mathrm{C}$ & 0.30667200 & -5.68361000 & -1.30361600 \\
\hline $\mathrm{C}$ & 1.58482400 & -4.39952900 & -2.91465900 \\
\hline $\mathrm{H}$ & 2.07561000 & -2.37317500 & -2.36306100 \\
\hline $\mathrm{H}$ & -0.14621700 & -6.62087900 & -0.99239600 \\
\hline $\mathrm{H}$ & 2.11706300 & -4.34709200 & -3.85930400 \\
\hline $\mathrm{H}$ & 0.99565500 & -6.44767700 & -3.19437800 \\
\hline $\mathrm{C}$ & -0.27784700 & -4.44067700 & 0.93103700 \\
\hline $\mathrm{H}$ & -0.10475300 & -5.32104400 & 1.55469600 \\
\hline $\mathrm{H}$ & -1.35275300 & -4.22376000 & 0.92447700 \\
\hline $\mathrm{C}$ & 0.93390000 & 0.70368800 & -1.02085800 \\
\hline 0 & 0.64714000 & 5000 & -0.57370800 \\
\hline $\mathrm{C}$ & 0.40990900 & 0.14369300 & -2.21533200 \\
\hline $\mathrm{H}$ & 0.95457200 & -0.72715800 & -2.57020900 \\
\hline $\mathrm{C}$ & 0.04888600 & 1.14411100 & -3.29879600 \\
\hline $\mathrm{H}$ & -0.65100200 & 1.89107700 & -2.91296100 \\
\hline $\mathrm{H}$ & -0.43278600 & 0.63678800 & -4.13965200 \\
\hline $\mathrm{H}$ & 0.93505100 & 1.66991900 & -3.67338300 \\
\hline $\mathrm{H}$ & -1.12709400 & -1.53956500 & -2.31040100 \\
\hline $\mathrm{C}$ & -1.21558800 & -0.58814800 & -1.78845500 \\
\hline $\mathrm{C}$ & -2.16852500 & -0.06925400 & -1.12519900 \\
\hline $\mathrm{C}$ & -2.77382200 & 0.89561200 & -0.22556400 \\
\hline 0 & -1.90662500 & 2.04413200 & 0.16047700 \\
\hline $\mathrm{H}$ & -1.25470900 & 2.19754500 & -0.55081600 \\
\hline $\mathrm{C}$ & -3.00319700 & 0.15836900 & 1.10782300 \\
\hline $\mathrm{C}$ & -3.34008200 & -1.32385400 & 3.47067000 \\
\hline $\mathrm{C}$ & -4.26507400 & -0.27862600 & 1.50947300 \\
\hline $\mathrm{C}$ & -1.89569800 & -0.15646800 & 1.92144900 \\
\hline $\mathrm{C}$ & -2.07281900 & -0.88900100 & 3.09612800 \\
\hline $\mathrm{C}$ & -4.44289700 & -1.01476600 & 2.67875300 \\
\hline $\mathrm{H}$ & -5.12327900 & -0.04330600 & 0.88676900 \\
\hline $\mathrm{H}$ & -1.20095500 & -1.09392000 & 3.71086500 \\
\hline $\mathrm{H}$ & -5.43710000 & -1.34138700 & 2.96645200 \\
\hline
\end{tabular}




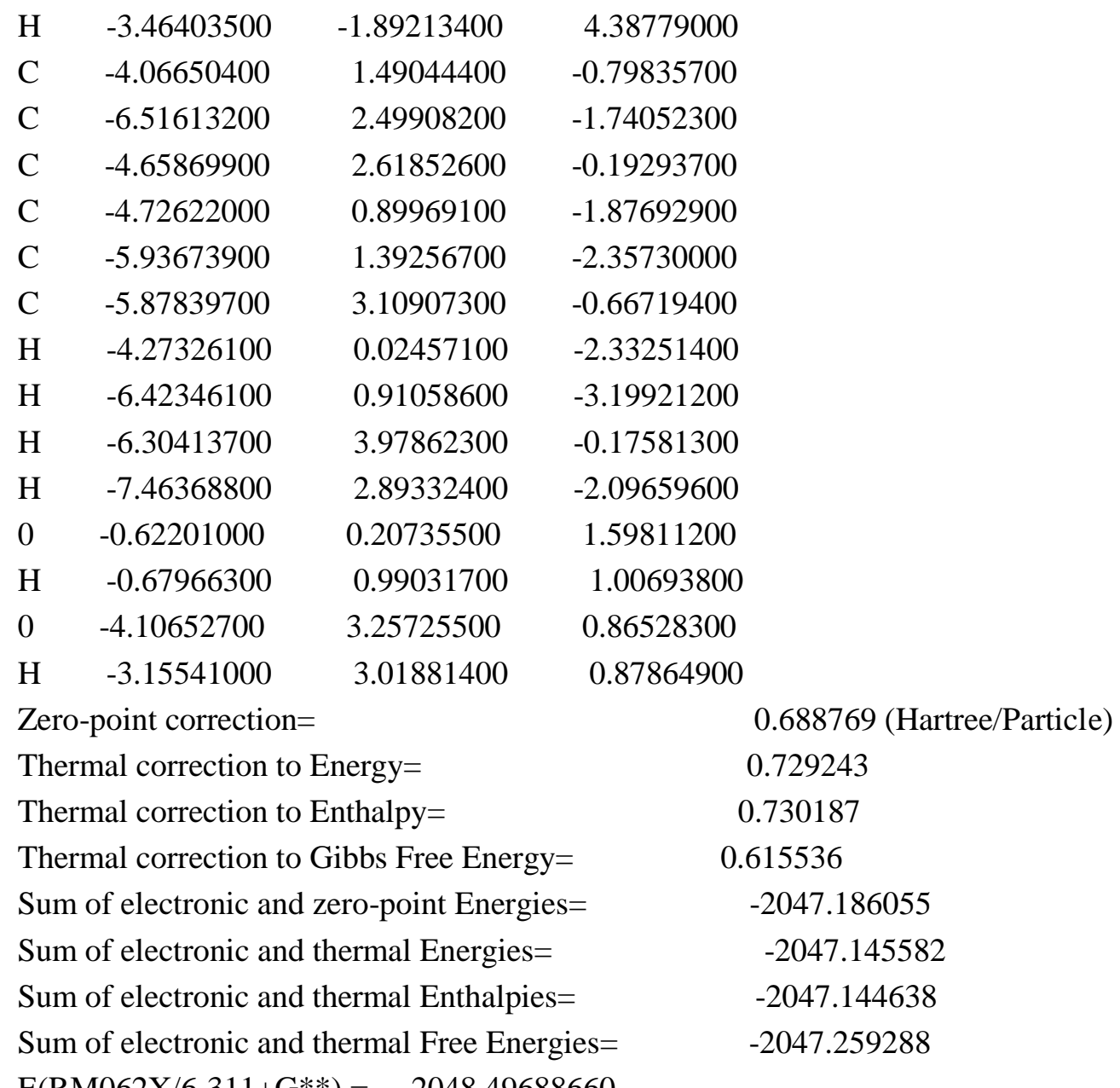

\subsubsection{Origin of Diastereoelectivity}

\begin{tabular}{lrrc}
\multicolumn{4}{l}{ MS-Cis-4 } \\
$\mathrm{C}$ & -1.53196100 & 1.73420000 & 0.74072200 \\
$\mathrm{O}$ & -1.26697400 & 1.47122800 & 1.87842800 \\
$\mathrm{C}$ & -0.95311100 & 2.83911000 & -0.11277200 \\
$\mathrm{C}$ & 0.07547700 & 3.64900800 & 0.66185400 \\
$\mathrm{H}$ & 0.89064000 & 3.01008000 & 1.01501600 \\
$\mathrm{H}$ & -0.37963800 & 4.12402500 & 1.53485100 \\
$\mathrm{H}$ & 0.49928900 & 4.42984800 & 0.02446400 \\
$\mathrm{C}$ & 0.50544100 & 0.97424000 & -0.93035900 \\
$\mathrm{C}$ & 0.24241300 & -0.28048100 & -0.44773700 \\
$\mathrm{C}$ & 1.54183300 & -0.82121600 & -0.06304000 \\
$\mathrm{C}$ & 4.27902000 & -1.11800000 & 0.42020500 \\
$\mathrm{C}$ & 2.00766300 & -1.99514800 & 0.54885400 \\
$\mathrm{C}$ & 2.47357000 & 0.17329700 & -0.37818200 \\
$\mathrm{C}$ & 3.84105000 & 0.06318100 & -0.16426700 \\
$\mathrm{C}$ & 3.37040500 & -2.12785300 & 0.77954200 \\
$\mathrm{H}$ & 1.32411400 & -2.77886000 & 0.85697400
\end{tabular}




$\begin{array}{lrrr}\mathrm{H} & 4.51915500 & 0.86605100 & -0.43264900 \\ \mathrm{H} & 3.74277800 & -3.03044100 & 1.25392800 \\ \mathrm{H} & 5.33790300 & -1.25715600 & 0.61378900 \\ \mathrm{C} & -1.03984600 & -1.00625000 & -0.31092900 \\ \mathrm{C} & -3.41534600 & -2.51219500 & 0.00533100 \\ \mathrm{C} & -2.27359800 & -0.39479900 & -0.02283500 \\ \mathrm{C} & -1.05366800 & -2.40177700 & -0.45849500 \\ \mathrm{C} & -2.21654900 & -3.14872400 & -0.30363200 \\ \mathrm{C} & -3.44099700 & -1.12827000 & 0.13969300 \\ \mathrm{H} & -0.13288300 & -2.90555700 & -0.73293500 \\ \mathrm{H} & -2.18415400 & -4.22567100 & -0.43352300 \\ \mathrm{H} & -4.35949200 & -0.59519800 & 0.36244600 \\ \mathrm{H} & -4.32880000 & -3.08423000 & 0.13311200 \\ 0 & 1.84215500 & 1.25798800 & -0.89931400 \\ \mathrm{C} & -0.35741400 & 2.11807000 & -1.35627700 \\ \mathrm{H} & -1.77541000 & 3.47857100 & -0.45494500 \\ \mathrm{H} & 0.24702900 & 2.82460300 & -1.93140500 \\ \mathrm{H} & -1.16533100 & 1.75845700 & -1.99594300 \\ 0 & -2.39536600 & 0.98167500 & -0.01651900\end{array}$

Zero-point correction $=$ 0.280803 (Hartree/Particle)
0.296420

Thermal correction to Energy= 0.297364

Thermal correction to Enthalpy= 0.238346

Thermal correction to Gibbs Free Energy= Sum of electronic and zero-point Energies= $-919.402886$

Sum of electronic and thermal Energies= $-919.387269$

Sum of electronic and thermal Enthalpies= $-919.386325$

Sum of electronic and thermal Free Energies= $-919.445343$

$\mathrm{E}(\mathrm{RM} 062 \mathrm{X} / 6-311+\mathrm{G}(2 \mathrm{~d}, \mathrm{p}))=-919.955286923$

\section{PS-Cis-4}

$\begin{array}{lrrr}\mathrm{C} & -2.05196300 & -1.62916700 & 0.91038800 \\ \mathrm{O} & -2.13533200 & -2.51437600 & 1.72116800 \\ \mathrm{C} & -1.99343800 & -1.93422400 & -0.58396100 \\ \mathrm{C} & -2.64800500 & -0.97085800 & -1.58021300 \\ \mathrm{H} & -2.00876900 & -0.13318700 & -1.86291600 \\ \mathrm{H} & -3.59056800 & -0.57190300 & -1.19353100 \\ \mathrm{H} & -2.87557000 & -1.53512800 & -2.48987200 \\ \mathrm{C} & 0.49668500 & -1.29597700 & -0.52404700 \\ \mathrm{C} & 0.49702500 & 0.01867800 & -0.16034600 \\ \mathrm{C} & 1.89186700 & 0.36269700 & 0.06232700 \\ \mathrm{C} & 4.66056700 & 0.25707500 & 0.30806800 \\ \mathrm{C} & 2.57967200 & 1.51628700 & 0.45807900 \\ \mathrm{C} & 2.61438700 & -0.80611700 & -0.18969300 \\ \mathrm{C} & 3.99499200 & -0.90086800 & -0.07820200\end{array}$




\begin{tabular}{cccc}
$\mathrm{C}$ & 3.96198500 & 1.44761900 & 0.57415900 \\
$\mathrm{H}$ & 2.04543600 & 2.43717500 & 0.67266400 \\
$\mathrm{H}$ & 4.51715200 & -1.82953300 & -0.28162100 \\
$\mathrm{H}$ & 4.51681200 & 2.32955400 & 0.87884400 \\
$\mathrm{H}$ & 5.74112900 & 0.23950000 & 0.40945300 \\
$\mathrm{C}$ & -0.60877700 & 0.99020200 & -0.09026600 \\
$\mathrm{C}$ & -2.65215300 & 2.93025000 & -0.01083400 \\
$\mathrm{C}$ & -1.79459900 & 0.77108200 & 0.61745700 \\
$\mathrm{C}$ & -0.47988500 & 2.21355500 & -0.76568100 \\
$\mathrm{C}$ & -1.48479200 & 3.17300200 & -0.73304300 \\
$\mathrm{C}$ & -2.80358100 & 1.72750900 & 0.66799900 \\
$\mathrm{H}$ & 0.42734500 & 2.39773400 & -1.33439800 \\
$\mathrm{H}$ & -1.35606700 & 4.10770400 & -1.26929300 \\
$\mathrm{H}$ & -3.69696400 & 1.50923900 & 1.24438300 \\
$\mathrm{H}$ & -3.44236200 & 3.67339200 & 0.02538400 \\
0 & 1.76631900 & -1.80884800 & -0.54320300 \\
$\mathrm{C}$ & -0.53741500 & -2.29793100 & -0.92640200 \\
$\mathrm{H}$ & -2.54910200 & -2.87401600 & -0.63964500 \\
$\mathrm{O}$ & -1.98587500 & -0.35960500 & 1.38892800 \\
$\mathrm{H}$ & -0.46166400 & -2.48685100 & -2.00492300 \\
$\mathrm{H}$ & -0.28313300 & -3.23793700 & -0.42440400 \\
\hline
\end{tabular}

Zero-point correction= 0.281078 (Hartree/Particle)

Thermal correction to Energy= 0.296787

Thermal correction to Enthalpy= 0.297731

Thermal correction to Gibbs Free Energy= 0.238218 Sum of electronic and zero-point Energies= $-919.398358$ Sum of electronic and thermal Energies= $-919.382649$ Sum of electronic and thermal Enthalpies= Sum of electronic and thermal Free Energies= $-919.381705$ $-919.441218$

\begin{tabular}{lrrr}
\multicolumn{4}{l}{ PS-trans-4 } \\
C & -1.77413800 & -1.21770000 & 0.96155800 \\
0 & -1.41497600 & -0.95918700 & 2.07392900 \\
$\mathrm{C}$ & -1.55392600 & -2.49307400 & 0.18223100 \\
$\mathrm{C}$ & -2.86459000 & -3.21132100 & -0.13938300 \\
$\mathrm{H}$ & -3.53085100 & -2.56068500 & -0.71336600 \\
$\mathrm{H}$ & -3.37940800 & -3.52149600 & 0.77418400 \\
$\mathrm{H}$ & -2.65873500 & -4.10467400 & -0.73616000 \\
$\mathrm{C}$ & 0.33203400 & -1.16013400 & -0.78765800 \\
$\mathrm{C}$ & 0.42495900 & 0.15390000 & -0.41320400 \\
$\mathrm{C}$ & 1.82872300 & 0.35459400 & -0.07022700 \\
$\mathrm{C}$ & 4.55239500 & -0.05744700 & 0.40028200 \\
$\mathrm{C}$ & 2.60314200 & 1.40632100 & 0.44297000
\end{tabular}




$\begin{array}{lrrr}\mathrm{C} & 2.45240000 & -0.87691600 & -0.29561800 \\ \mathrm{C} & 3.80292500 & -1.12132600 & -0.08483700 \\ \mathrm{C} & 3.95531700 & 1.18598700 & 0.66839000 \\ \mathrm{H} & 2.16078600 & 2.36788200 & 0.67991300 \\ \mathrm{H} & 4.23621700 & -2.09622700 & -0.28049400 \\ \mathrm{H} & 4.56480400 & 1.99113600 & 1.06672600 \\ \mathrm{H} & 5.61299400 & -0.19313300 & 0.58670600 \\ \mathrm{C} & -0.61205800 & 1.20546200 & -0.32813400 \\ \mathrm{C} & -2.48682100 & 3.31405800 & -0.11147800 \\ \mathrm{C} & -1.95134300 & 0.97311400 & 0.03357400 \\ \mathrm{C} & -0.25979200 & 2.53622700 & -0.60018500 \\ \mathrm{C} & -1.17513700 & 3.57797700 & -0.49572600 \\ \mathrm{C} & -2.87453300 & 2.00389200 & 0.14740500 \\ \mathrm{H} & 0.75079600 & 2.74897900 & -0.93234300 \\ \mathrm{H} & -0.86287900 & 4.59211500 & -0.72315200 \\ \mathrm{H} & -3.89265200 & 1.75764500 & 0.43049400 \\ \mathrm{H} & -3.20987300 & 4.11861000 & -0.02263200 \\ \mathrm{O} & 1.54294900 & -1.79017900 & -0.72532800 \\ \mathrm{C} & -0.81306700 & -2.06449500 & -1.11103400 \\ \mathrm{H} & -0.90659100 & -3.13126400 & 0.79008600 \\ \mathrm{O} & -2.43430400 & -0.31472800 & 0.16231000 \\ \mathrm{H} & -1.51820400 & -1.55878800 & -1.77582200 \\ \mathrm{H} & -0.43469600 & -2.95206600 & -1.62498900\end{array}$

Zero-point correction=

0.280928 (Hartree/Particle)

Thermal correction to Energy=

0.296621

Thermal correction to Enthalpy=

0.297565

Thermal correction to Gibbs Free Energy=

0.238397

Sum of electronic and zero-point Energies=

$-919.401106$

Sum of electronic and thermal Energies=

$-919.385414$

Sum of electronic and thermal Enthalpies=

$-919.384470$

Sum of electronic and thermal Free Energies=

$-919.443638$

$\mathrm{E}(\mathrm{RM} 062 \mathrm{X} / 6-311+\mathrm{G}(2 \mathrm{~d}, \mathrm{p}))=-919.954189551$

TS-M-cis2trans

$\begin{array}{lrrr}\mathrm{H} & -0.16055700 & -2.92101600 & -1.05898100 \\ \mathrm{C} & -1.05711400 & -2.46131500 & -0.65159600 \\ \mathrm{C} & -3.33686600 & -1.24386500 & 0.34839500 \\ \mathrm{C} & -0.96247900 & -1.14622000 & -0.17292100 \\ \mathrm{C} & -2.25613800 & -3.16059100 & -0.63601100 \\ \mathrm{C} & -3.40435900 & -2.53985500 & -0.14421700 \\ \mathrm{C} & -2.12385000 & -0.55360200 & 0.34893400 \\ \mathrm{H} & -2.29829700 & -4.17642400 & -1.01502000 \\ \mathrm{H} & -4.35313100 & -3.06712600 & -0.13387800 \\ \mathrm{H} & -4.20983300 & -0.75277300 & 0.76570200\end{array}$




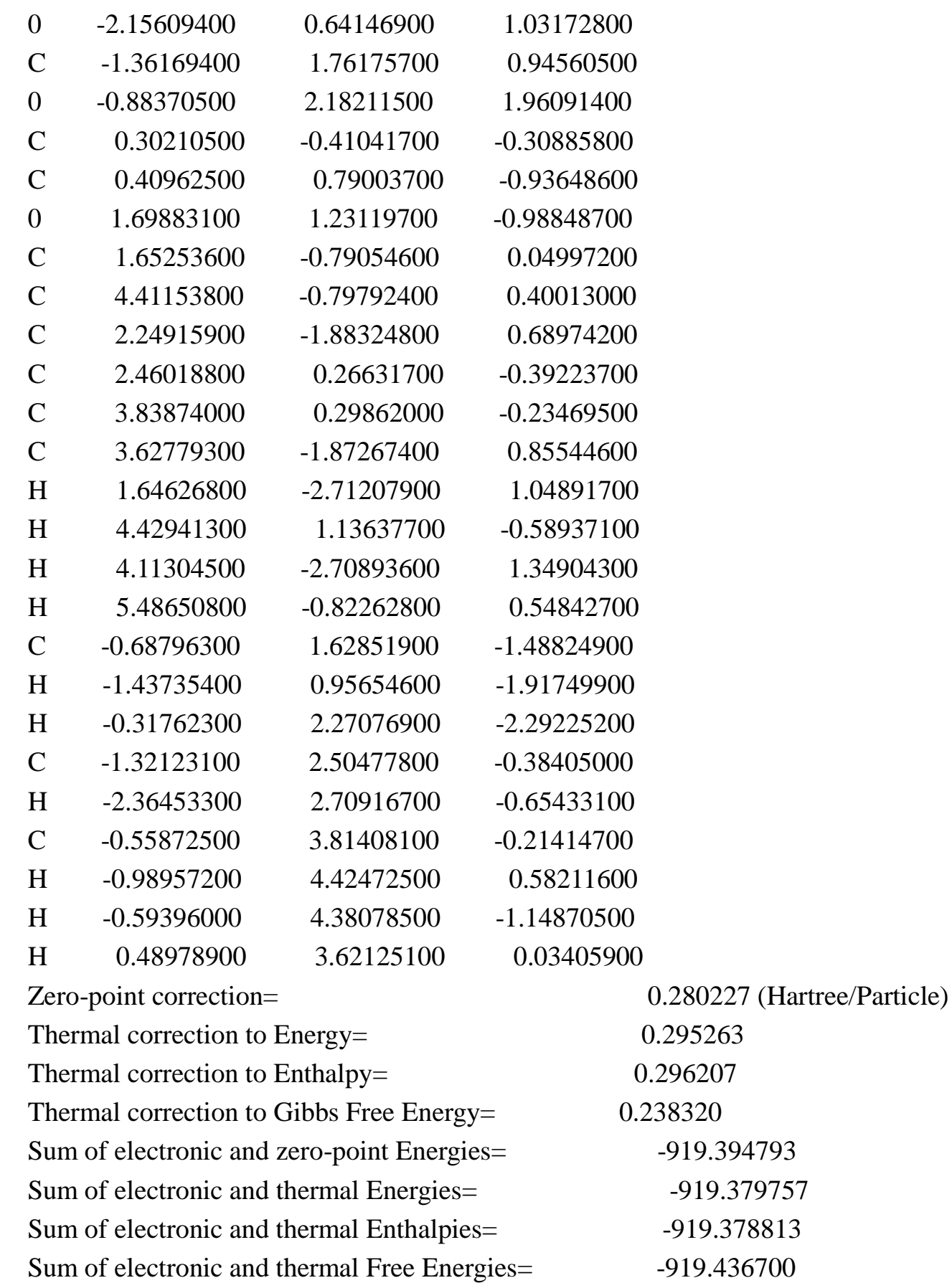

$\mathrm{E}(\mathrm{RM} 062 \mathrm{X} / 6-311+\mathrm{G}(2 \mathrm{~d}, \mathrm{p}))=-919.947484549$

\section{TS-PC2MT}

$\begin{array}{lrrr}\mathrm{H} & 1.00352700 & 2.69852600 & 0.49796400 \\ \mathrm{C} & -0.00850600 & 2.52468600 & 0.16968200 \\ \mathrm{C} & -2.67745100 & 2.16223100 & -0.46663100 \\ \mathrm{C} & -0.42779600 & 1.22139700 & -0.14072500 \\ \mathrm{C} & -0.87032300 & 3.61699500 & 0.12166600 \\ \mathrm{C} & -2.20609300 & 3.44848900 & -0.22419900 \\ \mathrm{C} & -1.80216400 & 1.09159800 & -0.40787800 \\ \mathrm{H} & -0.48760700 & 4.60289900 & 0.36563300\end{array}$




\begin{tabular}{|c|c|c|c|}
\hline $\mathrm{H}$ & -2.88194900 & 4.29624000 & -0.26954300 \\
\hline $\mathrm{H}$ & -3.72222900 & 1.96557000 & -0.68385500 \\
\hline & -2.30228000 & -0.18561600 & -0.58553800 \\
\hline & -2.41966500 & -0.90444400 & 0.58214200 \\
\hline & -2.80052100 & -0.42169400 & 1.61061900 \\
\hline $\mathrm{C}$ & 0.53559300 & 0.08539500 & -0.14664200 \\
\hline 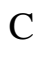 & 0.34278100 & -1.28045900 & -0.26381300 \\
\hline & 1.52530900 & -1.95581800 & -0.15229700 \\
\hline $\mathrm{C}$ & 1.99516300 & 0.24564300 & -0.01881200 \\
\hline C & 4.75513900 & -0.32507900 & 0.1734860 \\
\hline$C$ & 2.93643700 & 1.29547500 & 0.0113380 \\
\hline $\mathrm{C}$ & 2.51826200 & -1.05160800 & -0.00573900 \\
\hline $\mathrm{C}$ & 3.86271200 & -1.38284700 & 0.10224700 \\
\hline $\mathrm{C}$ & 4.28867900 & 0.99686300 & 0.1116090 \\
\hline $\mathrm{H}$ & 2.65678400 & 2.33532100 & -0.0807720 \\
\hline $\mathrm{H}$ & 4.18067000 & -2.41980600 & 0.1132970 \\
\hline $\mathrm{H}$ & 5.00362400 & 1.81349200 & 0.131667 \\
\hline $\mathrm{H}$ & 5.81950900 & -0.52039000 & 0.2556210 \\
\hline $\mathrm{C}$ & -0.75499400 & -2.26231400 & -0.60288200 \\
\hline $\mathrm{H}$ & -0.28706300 & -3.24962900 & -0.62450000 \\
\hline $\mathrm{H}$ & -1.11574800 & -2.05272300 & -1.61406100 \\
\hline $\mathrm{C}$ & -1.95494100 & -2.31523500 & $0.3506570 \mathrm{C}$ \\
\hline $\mathrm{H}$ & -1.65414200 & -2.70613600 & 1.32716500 \\
\hline $\mathrm{C}$ & -3.08217600 & -3.17764900 & -0.22643900 \\
\hline $\mathrm{H}$ & -3.92522500 & -3.23281300 & 0.4674020 \\
\hline $\mathrm{H}$ & -2.71640800 & -4.19280100 & -0.4057450 \\
\hline $\mathrm{H}$ & -3.43774200 & -2.76755500 & -1.1769500 \\
\hline
\end{tabular}

Zero-point correction=

Thermal correction to Energy=

0.280841 (Hartree/Particle)
0.295829
0.296773
0.238949
-919.391118
-919.376131
-919.375187
-919.433010

Thermal correction to Enthalpy=

Thermal correction to Gibbs Free Energy=

Sum of electronic and zero-point Energies=

Sum of electronic and thermal Energies=

Sum of electronic and thermal Enthalpies=

Sum of electronic and thermal Free Energies $=$

$\mathrm{E}(\mathrm{RM} 062 \mathrm{X} / 6-311+\mathrm{G}(2 \mathrm{~d}, \mathrm{p}))=-919.944082575$

TS-P-cis2trans

$\begin{array}{lrrr}\mathrm{H} & 0.09442600 & 2.60598900 & -1.46897500 \\ \mathrm{C} & -0.75176000 & 2.30394100 & -0.85799300 \\ \mathrm{C} & -2.90834500 & 1.50993100 & 0.70683100 \\ \mathrm{C} & -0.68501900 & 1.07532100 & -0.18659800 \\ \mathrm{C} & -1.87109600 & 3.12109600 & -0.75765600 \\ \mathrm{C} & -2.95454400 & 2.71900300 & 0.02398300\end{array}$




$\begin{array}{lccc}\mathrm{C} & -1.78195900 & 0.69788500 & 0.59801800 \\ \mathrm{H} & -1.90003700 & 4.06747200 & -1.28786500 \\ \mathrm{H} & -3.83392200 & 3.34964700 & 0.10815300 \\ \mathrm{H} & -3.72930000 & 1.18195200 & 1.33663900 \\ \mathrm{O} & -1.73811900 & -0.40745100 & 1.42491800 \\ \mathrm{C} & -1.47814400 & -1.69387600 & 1.04755600 \\ \mathrm{O} & -1.09165900 & -2.44551400 & 1.90156100 \\ \mathrm{C} & 0.49714900 & 0.20784400 & -0.31523600 \\ \mathrm{C} & 0.48114700 & -1.05105300 & -0.82571100 \\ \mathrm{O} & 1.72772600 & -1.60548300 & -0.85380100 \\ \mathrm{C} & 1.88121600 & 0.49200300 & -0.00185500 \\ \mathrm{C} & 4.63336700 & 0.27812500 & 0.32966900 \\ \mathrm{C} & 2.58232200 & 1.57844200 & 0.53446300 \\ \mathrm{C} & 2.58291500 & -0.66863200 & -0.35372700 \\ \mathrm{C} & 3.95530500 & -0.81327600 & -0.20127000 \\ \mathrm{C} & 3.95642800 & 1.45665400 & 0.69130000 \\ \mathrm{H} & 2.06190600 & 2.48801100 & 0.81965500 \\ \mathrm{H} & 4.46273100 & -1.72987800 & -0.48215500 \\ \mathrm{H} & 4.52297900 & 2.28546600 & 1.10432000 \\ \mathrm{H} & 5.70813100 & 0.21800000 & 0.46898300 \\ \mathrm{C} & -0.65677500 & -1.83617800 & -1.38709700 \\ \mathrm{H} & -0.26371300 & -2.76633200 & -1.80208500 \\ \mathrm{H} & -1.08365600 & -1.26497000 & -2.21967300 \\ \mathrm{C} & -1.79395100 & -2.15804700 & -0.37042300 \\ \mathrm{H} & -1.82911200 & -3.24284000 & -0.25129500 \\ \mathrm{C} & -3.16431900 & -1.68498900 & -0.86097800 \\ \mathrm{H} & -3.94038400 & -1.86826100 & -0.11127700 \\ \mathrm{H} & -3.43125800 & -2.24070500 & -1.76483000 \\ \mathrm{H} & -3.16600200 & -0.62114300 & -1.11068300 \\ \mathrm{Ze} & -p 0 i t 50\end{array}$

Zero-point correction $=$

0.280348 (Hartree/Particle)

Thermal correction to Energy=

0.295488

Thermal correction to Enthalpy=

0.296433

Thermal correction to Gibbs Free Energy=

0.238224

Sum of electronic and zero-point Energies=

$-919.395796$

Sum of electronic and thermal Energies=

$-919.380656$

Sum of electronic and thermal Enthalpies=

$-919.379711$

Sum of electronic and thermal Free Energies=

$-919.437920$

$\mathrm{E}(\mathrm{RM} 062 \mathrm{X} / 6-311+\mathrm{G}(2 \mathrm{~d}, \mathrm{p}))=-919.949520677$

\section{TS-PT2MC}

$\begin{array}{lrrr}\mathrm{H} & -0.60604000 & 2.44029100 & 1.16873500 \\ \mathrm{C} & 0.31608700 & 2.29569900 & 0.61948300 \\ \mathrm{C} & 2.76687400 & 1.92196600 & -0.63529600 \\ \mathrm{C} & 0.54587000 & 1.07512800 & -0.03409500\end{array}$




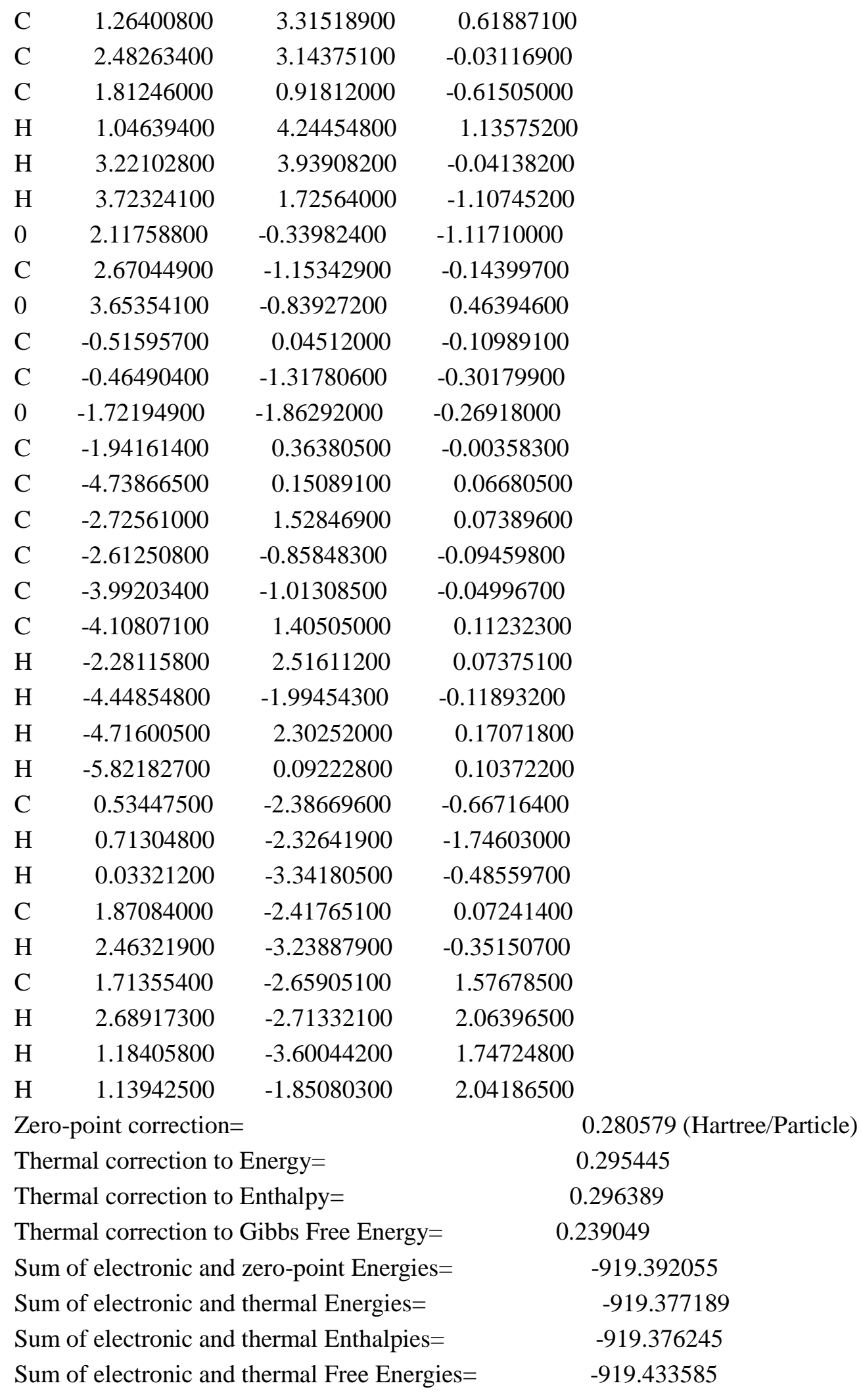

